\title{
Safety and Feasibility Of Ultrasound Accelerated Catheter Directed Thrombolysis And The Postthrombotic Syndrome
}

Citation for published version (APA):

Strijkers, R. H. W. (2016). Safety and Feasibility Of Ultrasound Accelerated Catheter Directed Thrombolysis And The Postthrombotic Syndrome. [Doctoral Thesis, Maastricht University]. Uitgeverij BOXPress. https://doi.org/10.26481/dis.20160113rs

Document status and date:

Published: 01/01/2016

DOI:

10.26481/dis.20160113rs

Document Version:

Publisher's PDF, also known as Version of record

Please check the document version of this publication:

- A submitted manuscript is the version of the article upon submission and before peer-review. There can be important differences between the submitted version and the official published version of record.

People interested in the research are advised to contact the author for the final version of the publication, or visit the DOI to the publisher's website.

- The final author version and the galley proof are versions of the publication after peer review.

- The final published version features the final layout of the paper including the volume, issue and page numbers.

Link to publication

\footnotetext{
General rights rights.

- You may freely distribute the URL identifying the publication in the public portal. please follow below link for the End User Agreement:

www.umlib.nl/taverne-license

Take down policy

If you believe that this document breaches copyright please contact us at:

repository@maastrichtuniversity.nl

providing details and we will investigate your claim.
}

Copyright and moral rights for the publications made accessible in the public portal are retained by the authors and/or other copyright owners and it is a condition of accessing publications that users recognise and abide by the legal requirements associated with these

- Users may download and print one copy of any publication from the public portal for the purpose of private study or research.

- You may not further distribute the material or use it for any profit-making activity or commercial gain

If the publication is distributed under the terms of Article $25 \mathrm{fa}$ of the Dutch Copyright Act, indicated by the "Taverne" license above, 
Safety And Feasibility Of Ultrasound Accelerated Catheter Directed Thrombolysis And The Postthrombotic Syndrome 
Cover: Anke den Boer

Lay-out: Rob Strijkers

Printing of this thesis was financially supported by: LAMEPRO B.V., MEDI NL, and BTG international group company.

(C) copyright Rob Hubertus Wilhelmus Strijkers, Rotterdam 2016

Printing: Proefschriftmaken.nl || Uitgeverij BOXPress

ISBN 97890 9029458-2 


\title{
Safety And Feasibility of Ultrasound Accelerated Catheter Directed Thrombolysis And The Postthrombotic Syndrome
}

\author{
PROEFSCHRIFT \\ ter verkrijging van de graad van doctor aan de Universiteit Maastricht, \\ op gezag van de Rector Magnificus, Prof dr. L.L.G. Soete \\ volgens het besluit van het College van Decanen, \\ in het openbaar te verdedigen \\ op woensdag 13 Januari 2016 om 12:00 uur
}

door

Rob Hubertus Wilhelmus Strijkers 


\section{Promotor}

Prof. dr. C.H.A. Wittens

Prof. dr. H. ten Cate

\section{Copromotor}

Dr. A.J. ten Cate-Hoek

\section{Beoordelingscommissie}

Prof. dr. P.M. Steijlen (voorzitter)

Prof. dr. M.W. de Haan

Prof. dr. M.V. Huisman (Leids Universitair Medisch Centrum)

Dr. R.J.M.W. Rennenberg

Dr. E.H. Serné (VuMC, Amsterdam) 
Aan mijn ouders 



\section{CONTENTS}

Chapter 1 General introduction 9

Chapter 2 Management of deep vein thrombosis and prevention of 25 post-thrombotic syndrome

Chapter 3 Villalta scale: Goals and limitations 41

Chapter $4 \quad$ Validation of the LET classification 51

Chapter 5 Proximal extension of deep venous thrombosis: does it 63 predict post-thrombotic syndrome and quality of life at long term?

Chapter 6 Feasibility of identifying deep vein thrombosis characteristics with contrast enhanced MR-Venography

Chapter 7 Safety and feasibility of ultrasound-accelerated catheterdirected thrombolysis in deep vein thrombosis

Chapter 8 Ultrasound-accelerated catheter-directed thrombolysis in acute Iliofemoral deep venous thrombosis

Chapter 9 Venous in-stent thrombosis treated by ultrasound accelerated catheter directed thrombolysis

Chapter 10 Fibrinogen levels in patients undergoing thrombolytic therapy for acute iliofemoral deep venous thrombosis are extremely method dependent

Chapter 11 Thrombectomy without lysis: the future?

Chapter 12 Summary and general discussion

Nederlandse samenvatting

Valorization

Dankwoord

List of publications

Curriculum vitae 
CHAPTER 1

General introduction 


\section{Deep venous thrombosis}

Deep venous thrombosis (DVT) of the lower extremities is a common disease in the western world, with an incidence of approximately $1-2$ per 1000 persons per year.(1) Patients who are diagnosed with DVT are immediately treated with low molecular heparin for at least 5 days and simultaneously with oral anticoagulation.(2) Anticoagulation is prescribed for 3-6 months depending on the risk factors, or lifelong in case of recurrent DVT or high risk for recurrence. As an alternative to the standard anticoagulation with vitamin K-antagonist, also direct oral factor $\mathrm{X}$ inhibitors are available as a treatment option. (3-5)

Additionally to anticoagulant treatment, therapeutic elastic stockings are used for 2 years in order to reduce the incidence of post thrombotic syndrome (PTS) by $50 \%$.(6) Patients are encouraged to resume their normal daily activities and can be safely treated ambulatory.(7) Regarding short-term results the fore mentioned therapy is excellent in preventing potentially lethal pulmonary emboli and preventing thrombus propagation.(8) However in the long term 20$50 \%$ of all DVT patients develop PTS. $(9,10)$ In case of iliofemoral DVT the estimated percentage of patients who will develop PTS is even higher and estimated to be over $50 \%$. Some reports even show PTS in as many as $80 \%$ of patients.(11) After cessation of anticoagulation, approximately $30 \%$ of patients develop a recurrent event of DVT within 5 years.(12)

\section{Iliofemoral DVT}

From previous studies it is known that patients with an iliofemoral DVT have a higher risk of developing PTS.(13-15) Approximately 1 in 4 of all patients has an iliofemoral DVT.(10,16) However, the definition of the extent of DVT is not well described in current literature.(17) Global terms like proximal DVT and distal DVT are used frequently, but further more specific descriptions are not available most of the time. This is largely due to the fact that the radiologist confirms the DVT via a two-point compression ultrasound. The two-point ultrasound only assesses the popliteal vein and the common femoral vein. If the thrombus is encountered, then the diagnosis is confirmed. Further visualization of the thrombus is unnecessary due to perceived lack of impact on treatment. This is not correct, because the recent expansion of treatment options makes complete visualization of the thrombus localization and extent more important. 


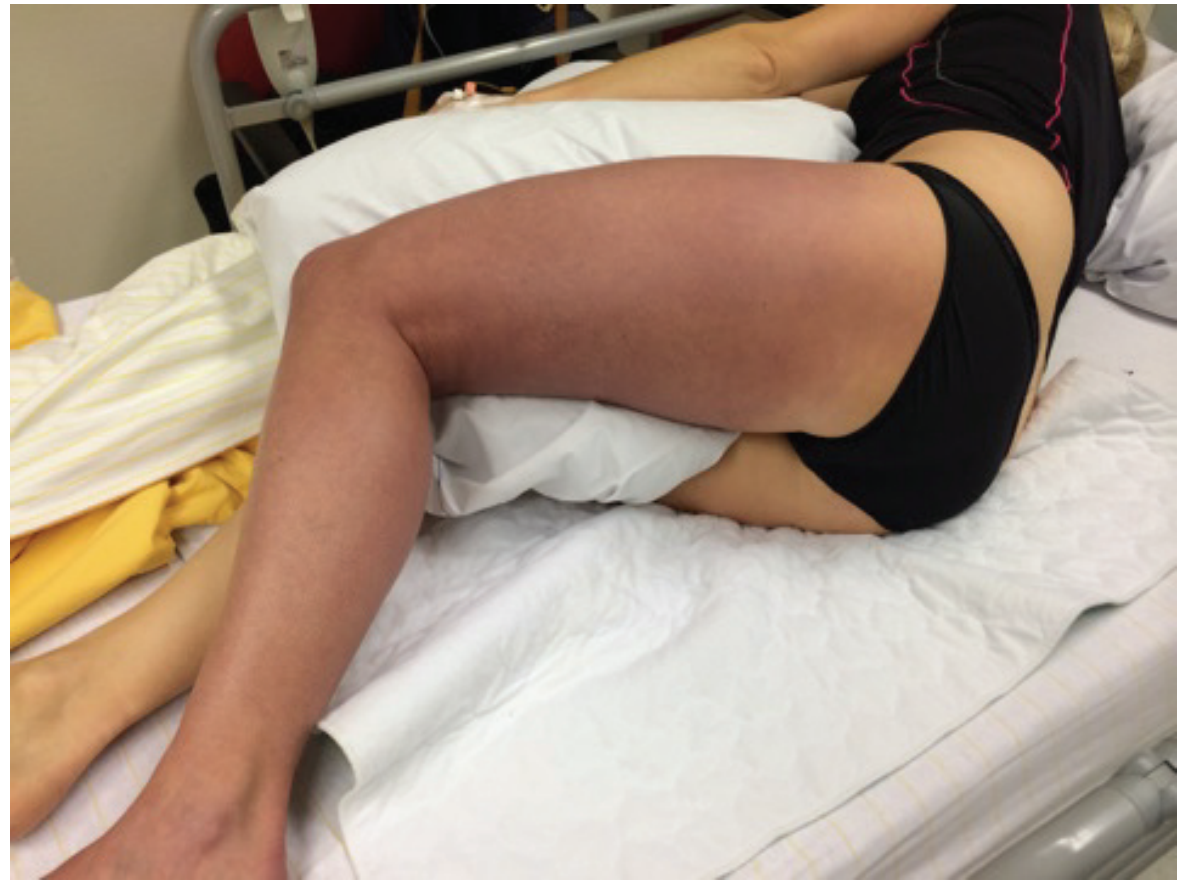

Picture of a female with an acute iliofemoral DVT of the left leg.

\section{Imaging of DVT}

Two-point ultrasonography (CUS) is the current standard procedure for the diagnosis of DVT.(18) Although CUS shows a high sensitivity for detecting DVT in the popliteal and femoral segment, the sensitivity for calf vein DVT and iliac tract DVT is far less.(19) Accurate imaging of the veins in the abdominal region is more difficult due to external interfering factors like bowel gasses or adipose tissue in case of increased BMI. Alternative imaging techniques such as computed tomography venography (CT-V) or Magnetic resonance venography (MRV) could be of use in these patients. $(20,21)$ The exact utility of these additional techniques in DVT diagnosis and treatment is still to be determined.

\section{The Post Thrombotic Syndrome (PTS)}

PTS is a chronic and debilitating condition, which occurs in $20-50 \%$ of patients after a DVT. PTS is the most frequent complication after DVT. Five to $10 \%$ of all cases of PTS will be severe, usually including venous ulcers.(22-24) The exact etiology of PTS is unknown but there is an association with reflux and obstruction of the deep venous system.(25) Standard anticoagulation does not actively dissolve thrombus. The fibrinolytic system is responsible for lysing the throm- 
bus and establishing recanalization of the vein. Natural recanalization observed with standard therapy is approximately $90 \%$ when the thrombus is confined to the popliteal and femoral veins. However when the iliac tract is also involved the percentage of natural recanalization drops to approximately 20-30\%.(1$5,26,27)$ In addition to fibrinolytic processes, inflammatory processes are initiated, leading to infiltration of neutrophils and macrophages into the thrombus and surrounding tissue. This inflammatory response causes thrombus remodeling and may as a side effect also cause destruction of the valves. (6,28-30) In $50 \%$ of patients natural recanalization is incomplete and the remaining thrombus is transformed into fibrous tissue (scar-tissue). (7-10,27,31) This may induce partial or total obstruction of the vein leading to an impaired outflow of blood out of the leg, with subsequent rise in intravenous pressure. Venous hypertension will induce incompetence of venous valves due to an increased hydrostatic pressure.(11,32) These three mechanisms induce impairment of outflow and ultimately lead to abnormal capillary perfusion. Decreased oxygenation will provoke impairment of the calf muscle pump and patients will experience complaints of pain in the calf. A number of risk factors have been associated with the development of PTS. Recurrent DVT and high BMI were the strongest predictors for PTS.(12,33) Other risk factors have not yet proven to be useful for selection of high-risk patients.(13-15,34)

\section{Diagnosis of PTS}

PTS is a syndrome and there is no gold standard test for its diagnosis. In some patients, acute symptoms of DVT like pain and swelling can last up to 3 or even 6 months. Therefore diagnosis of PTS should only be made starting from 6 months after the acute DVT event. $(10,16,35,36)$ The diagnosis of PTS should incorporate both clinical signs as well as patient complaints. A number of clinical scales are available for the diagnosis of PTS. The Villalta scale, the Venous Clinical severity score (VCSS) and CEAP classification are the most used scores. The Villalta scale is the preferred scale for the diagnosis of PTS. $(17,37)$ The CEAP classification is also frequently used in common practice, but does not include patient symptoms. $(18,38)$ The Villalta scale consists of 6 clinical signs and 5 patient symptoms. All items are scored from 0-3 and a maximum score of 33 can be reached. A score of $\geq 5$ translates into the diagnosis of PTS. There are subdivisions for PTS severity. From 5-9 points PTS is considered to be mild, from 10-14 PTS is moderate, and severe PTS is diagnosed when the score is 15 points or more or if a venous ulcer is present. $(19,39)$ The randomized controlled CaVent study used the Villalta-scale for PTS diagnosis, as do the currently enrolling Attract and CAVA trial. $(20,21,40,41)$ Imaging techniques are not used for the diagnosis of PTS because incompetence and obstruction can be 
visible in the absence of complaints of the patient or clinical signs at the site of the leg. There are many scoring systems for PTS in use. The Ginsberg measure defines PTS by the presence of daily leg pain and swelling that persists for at least 1 month, is should be typical in character (worse with standing or walking and relieved by rest or leg elevation), and occurs at least 6 months after acute DVT. It does not rate the severity of PTS, but correlates well with QoL scores and identifies only the more severe PTS in contrast to the Villalta scale.(22-24) Potential shortcomings of the Ginsberg scale therefore include a lack of sensitivity for milder forms of PTS. The Brandjes scale, scores PTS with clinical and subjective criteria comparable to the Villalta scale, but also includes the measured leg circumference. The patient should be assessed twice at least 3 months apart. The score has a subdivision of mild, moderate and severe PTS. $(23,25)$ The CEAP classification was designed to assess signs of primary chronic venous disease. The scale consists of 7 stages C0-6. C0 is no sings of venous disease on the leg, $\mathrm{C} 1$ is teleangectasia or reticulary veins, $\mathrm{C} 2$ are varicose veins, C3 edema, C4a hyperpigmentation or eczema, C4b lipodermatosclerosis or athrophy blanche, C5 healed venous ulcer, C6 active venous ulcer. The scale does not incorporate patient's complaints and is not specifically designed for PTS.(42) There is no agreed cut-off point for PTS and therefore it is not ideal for diagnostic use for PTS, however it has been used in previous studies. The Widmer classification has also been used for the diagnosis of PTS and to evaluate the therapeutic effect of compressive stockings on PTS.(43) Table 1 gives an overview of all the available scores and their properties.

\begin{tabular}{|c|c|c|c|}
\hline Scales & $\begin{array}{l}\text { Clinical signs } \\
\text { included? }\end{array}$ & $\begin{array}{l}\text { Patient symptoms } \\
\text { included? }\end{array}$ & $\begin{array}{l}\text { Specific for } \\
\text { PTS? }\end{array}$ \\
\hline CEAP & Yes & No & No \\
\hline VCSS & Yes & Yes & No \\
\hline $\begin{array}{l}\text { Widmer classifi- } \\
\text { cation }\end{array}$ & Yes & No & No \\
\hline Brandjes score & Yes & Yes & Yes \\
\hline Ginsberg score & Yes & Yes & Yes \\
\hline Villalta scale & Yes & Yes & Yes \\
\hline
\end{tabular}




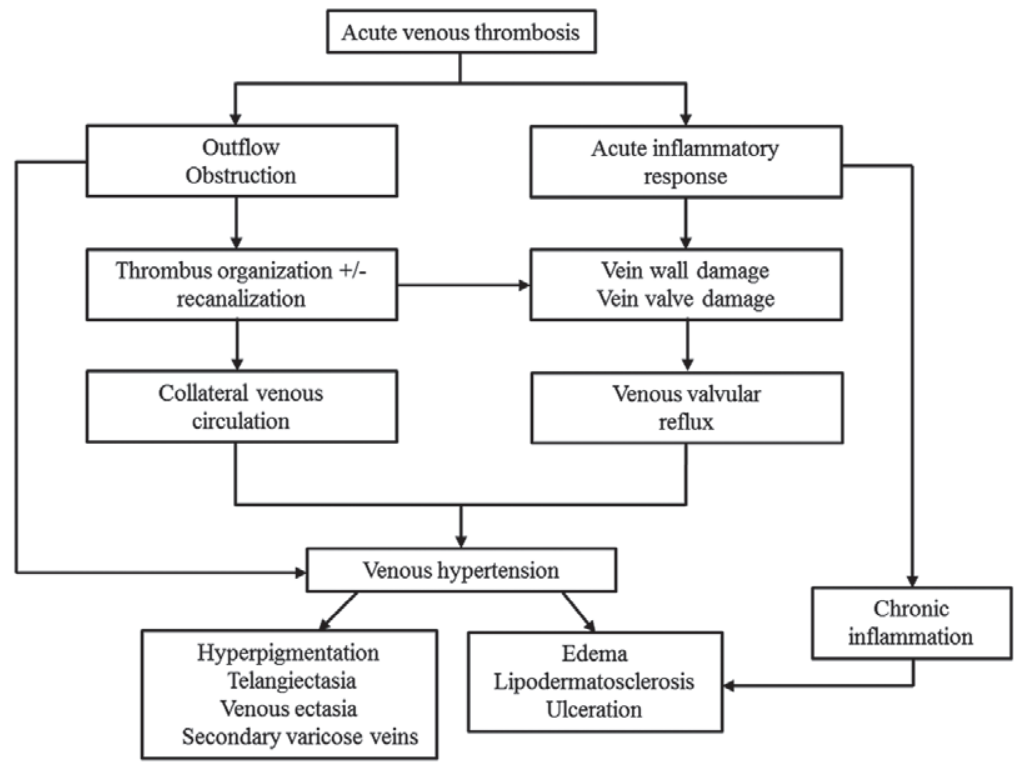

(44)

\section{Prevention of PTS}

Compression stockings are routinely prescribed for the prevention of PTS. In the past Prandoni and Brandjes showed a relative reduction in PTS of 50\% with the use of compression stockings in the DVT leg for 2 years. $(6,23)$ However in the recently published SOX-trial by Kahn et al. this finding could not be reproduced.(45) In fact the SOX-trial showed no effect of therapeutic elastic stockings on the prevention of PTS compared to sham stockings. The contradicting trials stirred up the debate on the routine use of compression stockings in DVT. Although compressive stockings seem to prevent PTS, the incidence is still high and alternative therapies to prevent PTS are badly needed. Because standard anticoagulation does not actively dissolve the thrombus, and residual obstruction and reflux are associated with PTS, active thrombus removal therapies are proposed as a treatment option potentially decreasing the PTS rate.

\section{Thrombus removal therapies}

In the eighties surgeons performed surgical thrombectomy on thrombi with an extension into the iliac tract with a risk of potentially causing pulmonary emboli or significant impairing the circulation in the leg. Via a groin incision the thrombus on the distal side was evacuated with strong compression on the leg. In this patient category a surprising lower incidence of PTS was witnessed.(46) 
This led to the idea that early thrombus removal in DVT patients could be beneficial for PTS prevention. However surgical thrombectomy is an invasive procedure and was associated with a high rate of recurrent DVT. Therefore this procedure nowadays is reserved for use in very specific cases such as acute phlegmasia cerulea dolens (picture 2).

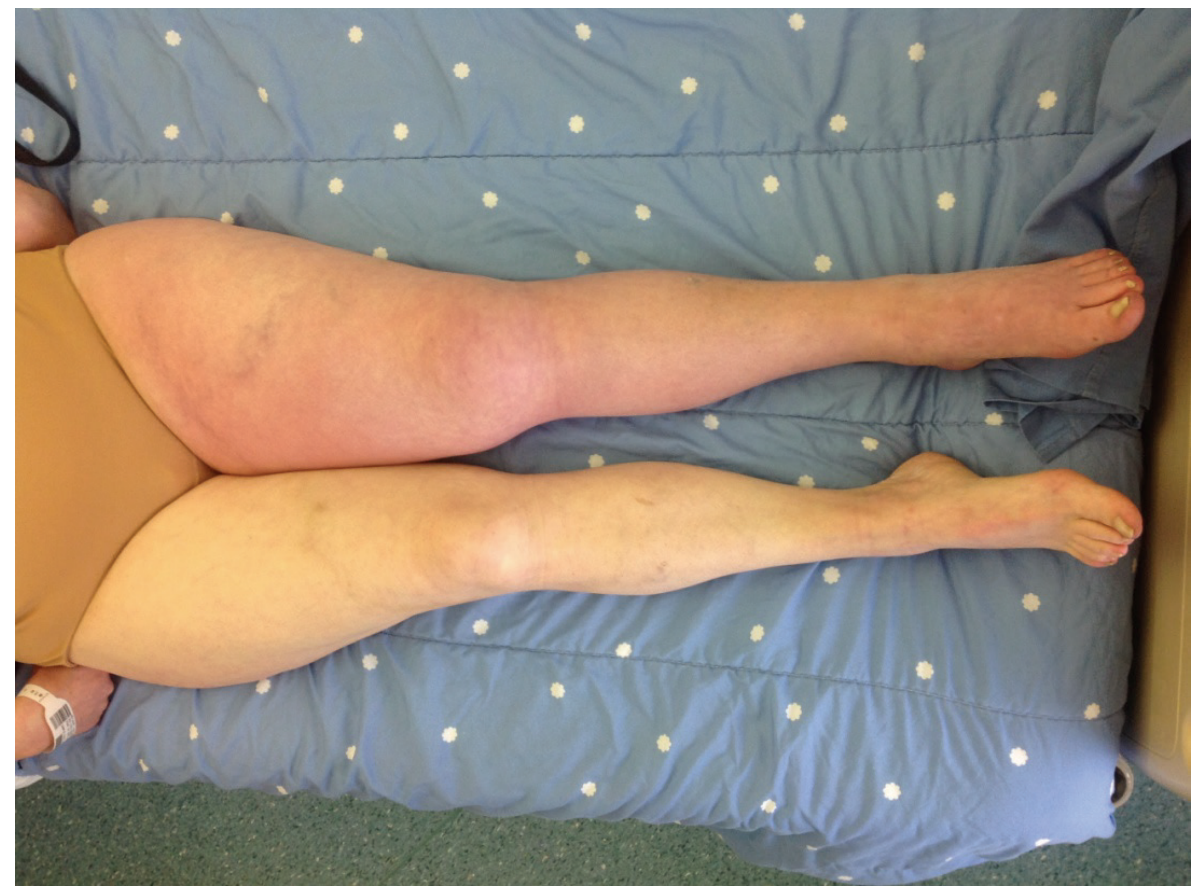

Example of Phlegmasia ceruleaa dolens. DVT with a complete venous outflow obstruction of the left leg.

\section{Systemic thrombolysis}

In the eighties and nineties research was performed on the use of thrombolysis for DVT. Via a peripheral arm infusion a large dose of thrombolytic drugs were infused to lyse the thrombus. Systemic thrombolysis was less invasive compared to surgical thrombectomy, however there were a few major drawbacks to this treatment: patients were hospitalized for up to seven days.(47) In order to achieve complete lysis of the thrombus, large doses of lytic drugs were required and a long infusion time.(48) Due to the large doses of thrombolytic drugs used and the prolonged treatment time of several days, systemic thrombolysis is associated with high bleeding risk and a number of major bleeding in critical organs i.e. intracranial hemorrhage. (49-52) Because of the enhanced bleeding risk, systemic thrombolysis is considered obsolete in the treatment of 
DVT. $(26,53)$ It is however still used in case of massive pulmonary embolism.(54)

In 1994 Semba et al. reported on the use of a multi sidehole catheter in combination with thrombolytic drugs.(55) The placement of the catheter through the thrombus allowed for more direct infusion of thrombolytic drug into the thrombus. The thrombus thus was more susceptible for lysis, and lower concentrations of lytic drugs could be used. The total time for complete lysis was reduced to 2-3 days. Major bleeding risk dropped to levels lower than 8\%. (56) In 2012 Enden et al. reported on the use of catheter directed thrombolysis in DVT for the prevention of PTS in a multicenter randomized controlled trial.(40) Compared to the standard conservative standard therapy, the group with additional CDT showed an absolute risk reduction of 14,4\% in the incidence of PTS. The CaVent study is the first randomized controlled trial to show the benefit of additional CDT to prevent PTS. Although the trial showed a positive result, many concerns regarding CDT for DVT still remain. The CaVent-study reported a median treatment time of 2.4 days, which is considered to be rather long.

\section{Mechanism of lysis}

A thrombus mainly consists of the three blood components: fibrin, platelets and red blood cells. In a case of vascular injury platelets are activated and form a primary platelet plug to close the injury and prevent blood loss. Simultaneously fibrinogen is activated by tissue factor release as a result from the injury, and is converted into fibrin. The formed fibrin strands will make up a matrix via crosslinking and interlinking with platelets and red blood cells and other blood components that are captured in the fibrin matrix. This process is useful in prevention of bleeding from wounds, however in the case of DVT the thrombus is formed unwanted and unneeded. The process of dissolving the thrombus by dissolving the fibrin matrix, which holds together the thrombus, is operated by plasmin. Urokinase and tissue plasminogen activators (TPA) activate plasminogen, and plasminogen is then converted to plasmin. Plasmin is able to cleave fibrin strands into fibrin degradation products. If the fibrin matrix is dissolved all the components of thrombus are released into the blood stream and the thrombus is resolved.(57) With CDT the thrombus is actively dissolved by infusing either urokinase, TPA or recombinant TPA into the thrombus.(58)

\section{Pharmacomechanical thrombolysis}

New techniques combined the standard infusion technique with a mechanical component. The addition of the mechanical components is aimed to reduce the total lysis time and so further reduce the bleeding risk associated with lysis. 
Three examples of Pharmacomechanical devices are: the trellis- 8 catheter, the angiojet powerpulse system, and the EKOS Endowave. (59-61)

\section{Ultrasound accelerated catheter directed thrombolysis (UACDT)}

The EKOS Endowave Peripheral Lysis System (EKOS Corporation, Bothell, WA) consists of a multilumen infusion catheter with a removable, coaxial ultrasound core and a control unit that simultaneously delivers high-frequency $(2.2 \mathrm{MHz})$, random low-energy $(0.45 \mathrm{~W})$ ultrasound energy and thrombolytic drug into the thrombus. The idea of adding high frequency ultrasound waves is to enhance lysis of the thrombus by better dispersion of the lytic drug into the thrombus and the ultrasound waves manipulated the fibrin strands to make them more susceptible for lysis. (62-64) These two mechanisms should allow for faster lysis of the thrombus and subsequent lower bleeding risk. The external device regulates the ultrasound waves formed in the catheter. The external device also regulates temperature. The ultrasound elements produce warmth in the catheter and in order to keep the temperature within body range the catheter is infused with a saline solution at room temperature. With a minimum continues rate of infusion at $35 \mathrm{cc} /$ hour it is able to keep the temperature from exceeding normal body limits. The infusion can be increased to a maximum of $100 \mathrm{cc} / \mathrm{hour}$ if necessary. The catheter is placed via a sheath in the popliteal or femoral vein and placed at the most cephalad point of the thrombus. In case of bilateral DVT two catheters, one in each leg can be placed. The maximum effective treatment duration of one catheter is 36 hours according to the manufacturer. In case of such a prolonged UACDT treatment time, new catheters should replace the catheter. During UACDT patients are anticoagulated with unfractionated heparin in a prophylactic dose at 1.2-1.7 times the APTT reference value. Simultaneously the urokinase or TPA is infused to lyse the thrombus. Treatment progress should be monitored at least once daily via phlebography and the patients should be monitored continuously on an intensive care ward or medium care setting, although never scientifically proven. Regular assessment of fibrinogen levels, hemoglobin values and Aptt should be performed and dosage of lytic drug of unfractionated heparin should be adjusted accordingly to ensure patient safety and limit the risk of bleeding.

\section{Patient selection and safety}

UACDT makes use of thrombolytic drugs. Although, originally the lytic drugs are given locally, after a while the drugs will diffuse and become systemic. The lytic drug will also be able to dissolve thrombus elsewhere in the body and can cause bleeding. Patients with enhanced bleeding risks are therefore no candi- 
dates for UACDT. Patients with recent surgery in the past six weeks or a history of bleeding in the past six months or gastro-intestinal bleeding as well as patients with a cerebrovascular incident in the past year have a strict contraindication for UACDT. The same applies for patients with active malignancies that are treated with surgery, chemo or radiotherapy or those hove have intracranial metastases are present. Patients with decreased kidney function or decreased liver function do also have a contraindication for UACDT, because of potentially prolonged bleeding time and the use of contrast fluids to monitor treatment progress. Pregnant women and women till two weeks post-partum are also excluded from thrombolysis. Furthermore patients who are wheelchair dependent are not good candidates for thrombolysis as the potential benefits of thrombolytic therapy do not outweigh the potential risks in this specific group.(65) To increase the success rate of UACDT it is important to assess the thrombus age. Currently the assessment of thrombus age is based on patient complaints only. This method is not ideal, and imaging techniques could prove useful in this matter. Because accurate assessment of thrombus age can aid in patient selection and potentially increase the success of thrombolysis. Patients with older thrombus age can then be spared the risk of UACDT.

\section{Aims and outline of this thesis}

In Chapter 2 an overview is given of the current treatment options for patients with an iliofemoral DVT. With this review of the current standard treatment options it becomes clear that PTS forms a substantial problem. PTS is a clinical diagnosis and there is no gold standard to diagnose PTS. IN 2008 a subcommittee of the ISTH suggested the standard use of the Villalta scale to be used in trials and the clinical setting. In Chapter $\mathbf{3}$ we took a closer look the Villalta scale, its properties, limitations, and alternative scales available for scoring PTS.

The best approach to reduce PTS incidence is prevention. If identification of high-risk PTS patients is possible in the acute phase, than additional treatments are possible to reduce PTS. In Chapter four we assessed the Lower Extremity Thrombosis (LET) Classification. The LET classification characterizes patients based on initial thrombus location and extent. We invited patients to fill-out questionnaires and assess their disease specific quality of life and PTS severity using the Villalta score. We evaluated the correlation between higher let classifications and PTS severity using the original extended version of the LET classification. In Chapter five we used the simplified version of the classification to lok at the effect on prediciting PTS severity using the same cohort. 
Thrombolysis of DVT is performed in the acute phase. Thrombus age is assessed by duration of complaints. In the literature the presence of complaints for two weeks is marked as acute DVT. Successful thrombolysis has been seen in thrombi as old as 3 to 4 weeks. There is no objective measurement available to predict successful thrombolysis. MR-venography could potentially be used to predict thrombus age. In Chapter six we assessed the reproducibility of predefined MR-venography findings by four experienced radiologists and the ability to distinguish acute from old thrombus.

Ultrasound accelerated catheter directed thrombolysis (UACDT) is a new therapy in the acute phase for iliofemoral DVT. Data regarding safety and feasibility are scarce. In Chapter seven reports on our earliest experience with UACDT for iliofemoral DVT and its safety and feasibility. In Chapter eight again safety and feasibility of UACDT for iliofemoral DVT is discussed and in addition the necessity of inserting stents afterwards in selected patients is considered.

In certain cases the stented venous tract occludes. In Chapter nine the possibility of regaining patency in patients with acute venous stent occlusion by way of UACDT was assessed. All patients are carefully monitored during thrombolysis and frequent routine measurements of fibrinogen are performed. In a number of cases we experienced difficulties determining accurate fibrinogen levels with the standard laboratory equipment. Safety in patients is of the utmost importance and cannot be guaranteed with unreliable measurements of fibrinogen. To try and optimize patient safety we looked at alternative methods for defining fibrinogen levels in patients undergoing UACDT for iliofemoral DVT and report on this in Chapter ten.

Chapter eleven gives an overview of the current thrombus removing therapies, imaging techniques in venous disease, and stenting. We conclude with recommendations for improvement of the current techniques and give our opinion on the ideal treatment options for patients with IFDVT in the near future. 


\section{References}

1. Nordström M, Lindblad B, Bergqvist D, Kjellström T. A prospective study of the incidence of deep-vein thrombosis within a defined urban population. J Intern Med. 1992 Aug;232(2):15560.

2. Kearon C, Akl EA, Comerota AJ, Prandoni P, Bounameaux H, Goldhaber SZ, et al. Antithrombotic therapy for VTE disease: Antithrombotic Therapy and Prevention of Thrombosis, $9^{\text {th }}$ ed: American College of Chest Physicians Evidence-Based Clinical Practice Guidelines. Chest. 2012 Feb;141(2 Suppl):e419S-94S.

3. EINSTEIN Investigators, Bauersachs R, Berkowitz SD, Brenner B, Büller HR, Decousus H, et al. Oral rivaroxaban for symptomatic venous thromboembolism. N Engl J Med. 2010 Dec 23;363(26):2499-510.

4. Hokusai-VTE Investigators, Büller HR, Decousus H, Grosso MA, Mercuri M, Middeldorp S, et al. Edoxaban versus warfarin for the treatment of symptomatic venous thromboembolism. $\mathrm{N}$ Engl J Med. 2013 Oct 10;369(15):1406-15.

5. Agnelli GG, Büller HR, Cohen A, Curto M, Gallus AS, Johnson M, et al. Oral apixaban for the treatment of acute venous thromboembolism. N Engl J Med. 2013 Aug 29;369(9):799-808.

6. Prandoni P, Lensing AWA, Prins MH, Frulla M, Marchiori A, Bernardi E, et al. Below-knee elastic compression stockings to prevent the post-thrombotic syndrome: a randomized, controlled trial. Ann Intern Med. 2004 Aug 17;141(4):249-56.

7. Partsch H. Compression and walking versus bed rest in the treatment of proximal deep venous thrombosis with low molecular weight heparin. Journal of Vascular Surgery. 2000.

8. Kearon C. Natural History of Venous Thromboembolism. Circulation. 2003 Jun 17;107(90231):22I--30.

9. Prandoni P. Post-thrombotic syndrome: prevalence, prognostication and need for progress. British Journal of Haematology. 2009.

10. Kahn SR, Shrier I, Julian JA, Ducruet T, Arsenault L, Miron MJ, et al. Determinants and time course of the postthrombotic syndrome after acute deep venous thrombosis. Ann Intern Med. 2008 Nov 18;149(10):698-707.

11. Kahn SR, Ginsberg JS. Relationship between deep venous thrombosis and the postthrombotic syndrome. Arch Intern Med. 2004 Jan 12;164(1):17-26.

12. Piccioli A, Prandoni P. Epidemiologic characteristics, management, and outcome of deep venous thrombosis in a tertiary-care hospital: the Brigham and Women's Hospital DVT registry. American Heart Journal. 1996.

13. Eichlisberger R, Frauchiger B, Widmer MT, Widmer LK, Jäger K. [Late sequelae of deep venous thrombosis: a 13-year follow-up of 223 patients]. VASA. 1994;23(3):234-43.

14. Tick LW, Doggen CJM, Rosendaal FR, Faber WR, Bousema MT, Mackaay AJC, et al. Predictors of the post-thrombotic syndrome with non-invasive venous examinations in patients 6 weeks after a first episode of deep vein thrombosis. Journal of Thrombosis and Haemostasis. 2010 Dec;8(12):2685-92.

15. Labropoulos N, Waggoner T, Sammis W, Samali S, Pappas PJ. The effect of venous thrombus location and extent on the development of post-thrombotic signs and symptoms. Journal of Vascular Surgery. 2008 Aug;48(2):407-12.

16. O'Donnell TF, Browse NL, Burnand KG, Thomas ML. The socioeconomic effects of an iliofemoral venous thrombosis. J Surg Res. 1977 May;22(5):483-8.

17. Vedantham S, Grassi CJ, Ferral H, Patel NH, Thorpe PE, Antonacci VP, et al. Reporting standards for endovascular treatment of lower extremity deep vein thrombosis. JVIR. 2009 Jul;20(7 Suppl):S391-408. 
18. Bates SM, Jaeschke R, Stevens SM, Goodacre S, Wells PS, Stevenson MD, et al. Diagnosis of DVT: Antithrombotic Therapy and Prevention of Thrombosis, 9th ed: American College of Chest Physicians Evidence-Based Clinical Practice Guidelines. Chest. 2012 Feb 6;141(2 suppl):e351Se418S.

19. Satin R, Tousignant L, Sheiner N. A prospective study comparing duplex scan and venography for diagnosis of lower-extremity deep vein thrombosis. Cardiovascular Surgery. 1996.

20. van Langevelde K, Tan M, Šrámek A, Huisman MV, de Roos A. Magnetic resonance imaging and computed tomography developments in imaging of venous thromboembolism. J Magn Reson Imaging. 2010 Nov 23;32(6):1302-12.

21. Baldt MM, Zontsich T, Stümpflen A, Fleischmann D, Schneider B, Minar E, et al. Deep venous thrombosis of the lower extremity: efficacy of spiral CT venography compared with conventional venography in diagnosis. Radiology. 1996 Aug;200(2):423-8.

22. van Gent WB, Wilschut ED, Wittens C. Management of venous ulcer disease. BMJ [Internet]. 2010 Nov 12;341(nov12 2):c6045-5. Available from: http://eutils.ncbi.nlm.nih.gov/entrez/eutils/elink.fcgi?dbfrom=pubmed\&id=21075818\&retmo de=ref\&cmd=prlinks

23. Brandjes DP, Büller HR, Heijboer H, Huisman MV, de Rijk M, Jagt H, et al. Randomised trial of effect of compression stockings in patients with symptomatic proximal-vein thrombosis. The Lancet. 1997 Mar;349(9054):759-62.

24. Ginsberg JS, Hirsh J, Julian J, Vander LaandeVries M, Magier D, MacKinnon B, et al. Prevention and treatment of postphlebitic syndrome: results of a 3-part study. Arch Intern Med. 2001 Sep 24;161(17):2105-9.

25. Ziegler S, Schillinger M, Maca TH, Minar E. Post-thrombotic syndrome after primary event of deep venous thrombosis 10 to 20 years ago. Thrombosis Research. 2001 Jan 15;101(2):23-33.

26. Comerota AJ, Aldridge SC. Thrombolytic therapy for deep venous thrombosis: a clinical review. Can J Surg. 1993 Aug;36(4):359-64.

27. Markel A, Meissner M, Manzo RA, Bergelin RO, Strandness DE. Deep venous thrombosis: rate of spontaneous lysis and thrombus extension. Int Angiol. 2003 Dec;22(4):376-82.

28. Fox E. The relationship between inflammation and venous thrombosis. Thromb Haemost. 2005.

29. Roumen-Klappe EM, Janssen MCH, van Rossum J, Holewijn S, van Bokhoven MMJA, Kaasjager K, et al. Inflammation in deep vein thrombosis and the development of post-thrombotic syndrome: a prospective study. Journal of Thrombosis and Haemostasis. 2009 Apr;7(4):582-7.

30. Shbaklo H, Holcroft CA, Kahn SR. Levels of inflammatory markers and the development of the post-thrombotic syndrome. Thromb Haemost. 2009 Feb 10.

31. Raju S, Davis M, Martin A. Assessment of residual thrombus after venous thrombolytic regimens. Journal of Vascular Surgery. Society for Vascular Surgery; 2014 Apr 1;2(2):148-54.

32. Latella J, Desmarais S, Miron Mj, Roussin A, Joyal F, Kassis J, et al. Relation between D-dimer level, venous valvular reflux and the development of post-thrombotic syndrome after deep vein thrombosis. Journal of Thrombosis and Haemostasis. 2010 Oct;8(10):2169-75.

33. Kahn SR, Shbaklo H, Lamping DL, Holcroft CA, Shrier I, Miron MJ, et al. Determinants of healthrelated quality of life during the 2 years following deep vein thrombosis. Journal of Thrombosis and Haemostasis. 2008 Jul;6(7):1105-12.

34. de Wolf MAF, Wittens CHA, Kahn SR. Incidence and risk factors of the post-thrombotic syndrome. Phlebology. 2012 Mar;27 Suppl 1:85-94.

35. Kahn SR, Partsch H, Vedantham S, Prandoni P, Kearon C, Subcommittee on Control of Anticoagulation of the Scientific and Standardization Committee of the International Society on Thrombosis and Haemostasis. Definition of post-thrombotic syndrome of the leg for use in clinical investigations: a recommendation for standardization. Journal of Thrombosis and Haemostasis. 2009 May; $7(5): 879-83$.

36. Kahn SR. The post-thrombotic syndrome: the forgotten morbidity of deep venous thrombosis. J Thromb Thrombolysis. 2006 Feb;21(1):41-8. 
37. Eklof B, Perrin M, Delis KT, Rutherford RB, Gloviczki P, American Venous Forum, et al. Updated terminology of chronic venous disorders: the VEIN-TERM transatlantic interdisciplinary consensus document. Journal of vascular surgery : official publication, the Society for Vascular Surgery [and] International Society for Cardiovascular Surgery, North American Chapter. 2009. pp. 498-501.

38. Eklof B, Rutherford RB, Bergan JJ, Carpentier PH, Gloviczki P, Kistner RL, et al. Revision of the CEAP classification for chronic venous disorders: consensus statement. 2004. pp. 1248-52.

39. Villalta S, Bagatella P, Piccioli A, Lensing A, Prins M, Prandoni P. Assessment of validity and reproducibility of a clinical scale for the post-thrombotic syndrome. Haemostasis; 1994;24(158a).

40. Enden T, Haig Y, Kløw N-E, Slagsvold C-E, Sandvik L, Ghanima W, et al. Long-term outcome after additional catheter-directed thrombolysis versus standard treatment for acute iliofemoral deep vein thrombosis (the CaVenT study): a randomised controlled trial. Lancet. 2012 Jan 7;379(9810):31-8.

41. Comerota AJ. The ATTRACT Trial: Rationale for Early Intervention for Iliofemoral DVT. Perspectives in Vascular Surgery and Endovascular Therapy. 2010 Jul 13;21(4):221-5.

42. Kolbach DN, Neumann HAM, Prins MH. Definition of the post-thrombotic syndrome, differences between existing classifications. European Journal of Vascular \& Endovascular Surgery. 2005 Oct;30(4):404-14.

43. Soosainathan A, Moore HM, Gohel MS, Davies AH. Scoring systems for the post-thrombotic syndrome. Journal of Vascular Surgery. 2013 Jan;57(1):254-61.

44. Kahn SR, Comerota AJ, Cushman M, Evans NS, Ginsberg JS, Goldenberg NA, et al. The Postthrombotic Syndrome: Evidence-Based Prevention, Diagnosis, and Treatment Strategies: A Scientific Statement From the American Heart Association. Circulation. 2014 Sep 22.

45. Kahn SR, Shapiro S, Wells PS, Rodger MA, Kovacs MJ, Anderson DR, et al. Compression stockings to prevent post-thrombotic syndrome: a randomised placebo-controlled trial. Lancet. 2014 Mar 8;383(9920):880-8.

46. Plate G, Eklof B, Norgren L, Ohlin P, Dahlström JA. Venous thrombectomy for iliofemoral vein thrombosis--10-year results of a prospective randomised study. European Journal of Vascular \& Endovascular Surgery. 1997 Nov;14(5):367-74.

47. Bounameaux H, Banga JD, Bluhmki E, Coccheri S, Fiessinger JN, Haarmann W, et al. Doubleblind, randomized comparison of systemic continuous infusion of 0.25 versus $0.50 \mathrm{mg} / \mathrm{kg} / 24 \mathrm{~h}$ of alteplase over 3 to 7 days for treatment of deep venous thrombosis in heparinized patients: results of the European Thrombolysis with rt-PA in Venous Thrombosis (ETTT) trial. Thromb Haemost. 1992 Mar 2;67(3):306-9.

48. Baldwin ZK, Comerota AJ, Schwartz LB. Catheter-directed thrombolysis for deep venous thrombosis. Vascular and Endovascular Surgery. 2004;38(1):1-9.

49. Janssen MCH, Wollersheim H, Schultze-Kool LJ, Thien T. Local and systemic thrombolytic therapy for acute deep venous thrombosis. Neth J Med. 2005 Mar;63(3):81-90.

50. Watson LI, Armon MP. Thrombolysis for acute deep vein thrombosis. Cochrane Database Syst Rev. 2004;(4):CD002783.

51. Forster A, Wells P. Tissue plasminogen activator for the treatment of deep venous thrombosis of the lower extremity: a systematic review. Chest. 2001 Feb;119(2):572-9.

52. Schweizer J, Schweizer J, MD, Kirch W, Kirch W, Koch R, et al. Short- and long-term results after thrombolytic treatment of deep venous thrombosis. J Am Coll Cardiol. 2000 0ct;36(4):133643.

53. Watson L, Broderick C, Armon MP. Thrombolysis for acute deep vein thrombosis. Cochrane Database Syst Rev. 2014;1:CD002783.

54. Jaff MR, McMurtry MS, Archer SL, Cushman M, Goldenberg N, Goldhaber SZ, et al. Management of Massive and Submassive Pulmonary Embolism, Iliofemoral Deep Vein Thrombosis, and Chronic Thromboembolic Pulmonary Hypertension: A Scientific Statement From the American Heart Association. Circulation. 2011 Apr 25;123(16):1788-830. 
55. Semba CP, Dake MD. Iliofemoral deep venous thrombosis: aggressive therapy with catheterdirected thrombolysis. Radiology. 1994 May;191(2):487-94.

56. Comerota AJ, Aldridge SC, Cohen G, Ball DS, Pliskin M, White JV. A strategy of aggressive regional therapy for acute iliofemoral venous thrombosis with contemporary venous thrombectomy or catheter-directed thrombolysis. Journal of Vascular Surgery. 1994 Aug;20(2):244-54.

57. Alkjaersig N, Fletcher AP, Sherry S. The mechanism of clot dissolution by plasmin. J Clin Invest. 1959 Jul;38(7):1086-95.

58. Ouriel K, Welch EL, Shortell CK, Geary K, Fiore WM, Cimino C. Comparison of streptokinase, urokinase, and recombinant tissue plasminogen activator in an in vitro model of venous thrombolysis. Journal of Vascular Surgery. 1995 Nov;22(5):593-7.

59. O'Sullivan GJ, Lohan DG, Gough N, Cronin CG, Kee ST. Pharmacomechanical thrombectomy of acute deep vein thrombosis with the Trellis-8 isolated thrombolysis catheter. JVIR. 2007 Jun;18(6):715-24.

60. Kasirajan K, Gray B, Ouriel K. Percutaneous AngioJet thrombectomy in the management of extensive deep venous thrombosis. JVIR. 2001 Feb;12(2):179-85.

61. Parikh S, Motarjeme A, McNamara T, Raabe R, Hagspiel K, Benenati JF, et al. Ultrasoundaccelerated Thrombolysis for the Treatment of Deep Vein Thrombosis: Initial Clinical Experience. Journal of Vascular and Interventional Radiology. 2008 Apr;19(4):521-8.

62. Francis CW, Blinc A, Lee S, Cox C. Ultrasound accelerates transport of recombinant tissue plasminogen activator into clots. Ultrasound Med Biol. 1995;21(3):419-24.

63. Siddiqi F, Blinc A, Braaten J, Francis CW. Ultrasound increases flow through fibring gels. Thromb Haemost. Schattauer; 1995;73(3):495-8.

64. Braaten JV, Goss RA, FRANCIS CW. Ultrasound reversibly disaggregates fibrin fibers. Thromb Haemost. 1997 Sep;78(3):1063-8.

65. Broholm R, Panduro Jensen L, Baekgaard N. Catheter-directed thrombolysis in the trea \tment of iliofemoral venous thrombosis. A review. Int Angiol. 2010 Aug;29(4):292-302. 


\section{CHAPTER 2}

\section{Management of deep vein throm- bosis and prevention of post- thrombotic syndrome}

Rob H W Strijkers

Arina J ten Cate-Hoek

Stephanie F F W Bukkems

Cees H A Wittens

British Medical Journal 2011;343:d5916 


\section{Introduction}

The annual global incidence of deep vein thrombosis (DVT) of the leg is 1.6 per $1000 .{ }^{1}$ Classically; venous thrombosis of a lower limb begins in a deep calf vein and propagates more proximally. Symptoms include swelling, pain, and redness of the leg, depending on the vein segment(s) involved (see table 1). ${ }^{2} \mathrm{~Pa}-$ tients are at risk of pulmonary embolism. ${ }^{3}$ Despite optimal conservative treatment with anticoagulation and compression, one in four patients develops a post-thrombotic syndrome within one year, ${ }^{2}$ and one in three develops a recurrent DVT within five years. ${ }^{4}$ Patients with post-thrombotic syndrome have poor quality of life. ${ }^{5}$ A more aggressive approach to treatment, such as removal of early thrombus using catheter directed thrombolysis, might improve outcomes for patients with DVT compared with standard anticoagulation treatment. ${ }^{4-12}$

\section{Who gets DVT?}

The formation of venous thrombosis depends on a triad of hypercoagulability, stasis, and interruption of the integrity of the vein wall. One or more factors may dominate, depending on the underlying risk factor. Table 2 outlines the known risk factors for DVT. ${ }^{6}$ The most common locations for venous thrombosis of the leg are shown in table $1 .^{2}$

Table 1

\begin{tabular}{|ll|}
\hline Location of the thrombus & Percentage of total deep vein thrombosis \\
\hline Distal veins & 40 \\
\hline Popliteal vein & 16 \\
\hline Femoral vein & 20 \\
\hline Common femoral vein & 20 \\
\hline Iliac vein & 4 \\
\hline
\end{tabular}

\section{How is DVT diagnosed?}

Patients with DVT usually present with pain, and swelling of the leg, varying degrees of redness, or muscle cramps. When DVT is confined to the popliteal vein the calf will be mostly affected. When DVT originates or extends more proximally to the iliofemoral vein segment, patients usually have swelling of the whole leg and more severe pain and redness. Mobility may be impaired because of heaviness and pain in the leg. In severe cases patients may develop phlegmasia cerulea dolens (fig 1). 


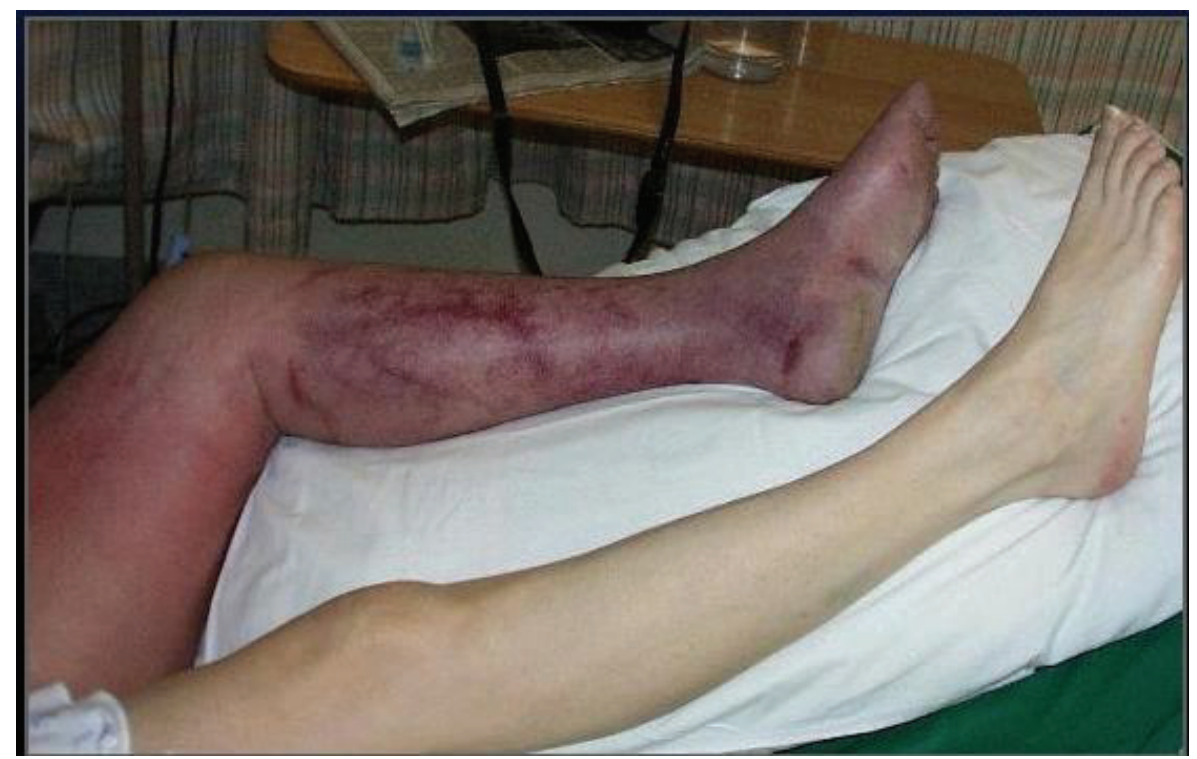

Fig 1 Phlegmasia cerulea dolens: the entire left leg is swollen and inflamed, with a blue-red aspect. The leg is very painful. It occurs when a deep vein thrombosis blocks the whole venous return of the leg. It can ultimately lead to gangrene of the leg

Because these clinical signs are not specific to DVT clinical scoring systems and diagnostic tests have been developed. The Wells score is the most widely validated method used to assess a patient's risk of current DVT (box). A Wells score of less than 2 means that the patient has a low risk of DVT, while those with a score of 2 or more are at high risk of current DVT.7 A D-dimer test demonstrates the presence of blood clot degradation products. It has a sensitivity of $95.3 \%$ and a specificity of $44.7 \%$ for DVT. The negative predictive value is high at $97.7 \%$, making it a useful test for ruling out DVT. ${ }^{8}$ A negative D-dimer blood test and a Wells score of less then 2 are effective in ruling out a DVT without the need for duplex ultrasound. ${ }^{9}$ 


\begin{tabular}{|ll|}
\hline \multicolumn{1}{|l|}{ Wells score for risk of deep venous thrombosis } \\
\hline $\mathbf{1}$ point & $\begin{array}{l}\text { active cancer }<6 \text { months or palliation } \\
\text { extremities }\end{array}$ \\
\hline $\mathbf{1}$ point & $\begin{array}{l}\text { recently bedridden for more than } 3 \text { days or major surgery, within } \\
\text { 4 weeks }\end{array}$ \\
\hline $\mathbf{1}$ point & $\begin{array}{l}\text { localised tenderness along the distribution of the deep venous } \\
\text { system }\end{array}$ \\
\hline $\mathbf{1}$ point & entire swollen leg \\
\hline $\mathbf{1}$ point & $\begin{array}{l}\text { calf swelling by more than } 3 \text { cm when compared with the asymp- } \\
\text { tomatic leg }\end{array}$ \\
\hline $\mathbf{1}$ point & pitting edema \\
\hline $\mathbf{1}$ point & collateral superficial veins (non-varicose) \\
\hline $\mathbf{1}$ point & previously documented DVT \\
\hline $\mathbf{- 2}$ points & $\begin{array}{l}\text { alternative diagnosis as likely or greater than that of deep vein } \\
\text { thrombosis. }\end{array}$ \\
\hline $\begin{array}{l}\text { total } \\
\text { score }\end{array}$ & \\
\hline$<2$ & low risk of DVT \\
\hline$\geq \mathbf{2}$ & high risk of DVT \\
\hline
\end{tabular}

In patients with clinical signs consistent with DVT and a Wells score of 2 or more or a positive D-dimer test (or both), imaging is used to confirm the diagnosis. Non-invasive two point duplex ultrasound is the current standard imaging technique. Duplex ultrasound has a sensitivity of $98.7 \%$ and specificity of $100 \%$ to detect or rule out an above knee thrombus, and a sensitivity of $85.2 \%$ and specificity of $98.2 \%$ for below knee DVT, when compared with the gold standard (invasive venography). ${ }^{10}$ Computed tomography venography and magnetic resonance venography can be used to image the exact extent of the DVT (fig 2). 11,12 


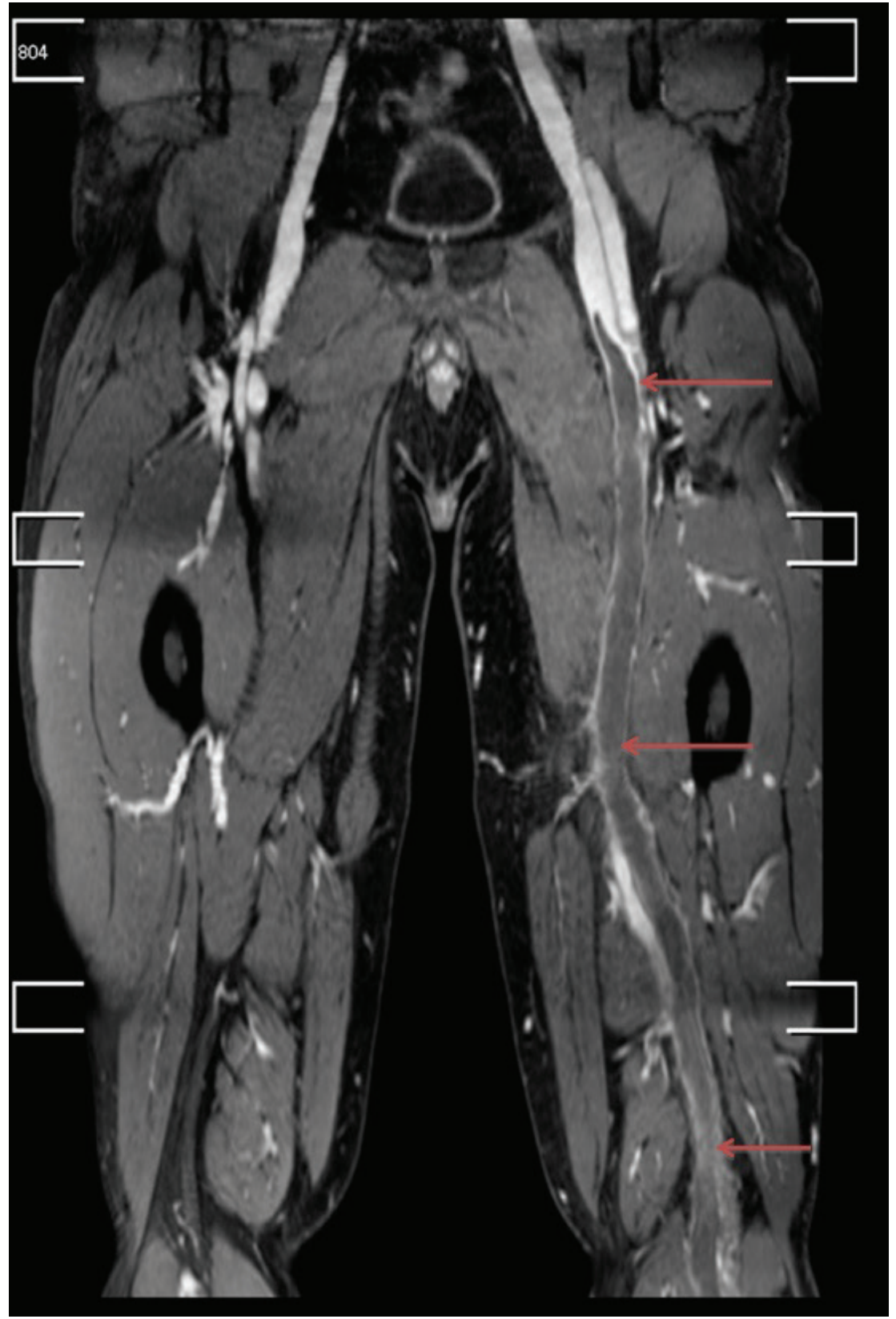

Fig 2 Magnetic resonance venogram showing a thrombus in the left leg extending from the popliteal vein to the common femoral vein; the red arrows point to the position of the thrombus in the vessel 


\section{Why is it important to treat DVT?}

\section{Risk of pulmonary embolism}

If DVT is left untreated about $50 \%$ of patients will develop a symptomatic pulmonary embolism, which carries a $10 \%$ risk of death within one hour of onset of initial symptoms. The main goal of treatment is to prevent pulmonary embolism, propagation of the clot, and recurrence of the DVT.13-15

\section{Risk of post-thrombotic syndrome}

In addition, $43-47 \%$ of patients develop post-thrombotic syndrome within two years of developing symptomatic DVT, although some studies have reported higher rates $(>60 \%)$ in patients with iliofemoral DVT.2,16 Post-thrombotic syndrome is a chronic debilitating condition caused by venous hypertension as a result of persistent obstruction of venous outflow and venous insufficiency. A recent observational study found a severely decreased quality of life in patients with post-thrombotic syndrome comparable to that of patients with chronic diseases such as diabetes, obstructive lung disease, and congestive heart failure. ${ }^{5}$ Patients present with a painful heavy leg and may also have cramps, paraesthesia, and pruritus. On examination the leg may be edematous with varicosities or hyperpigmentation of the skin (or both). The condition can be classified according to the recently developed and validated Villalta scale (figure 3). ${ }^{17}$ A retrospective study estimated that $30 \%, 10 \%$, and $3 \%$ of people with DVT develop mild, moderate, and severe post-thrombotic syndrome, respectively, and venous obstruction combined with reflux increased the risk significantly. ${ }^{18}$ A more severe post-thrombotic syndrome at one month, more extensive DVT (iliofemoral versus calf DVT), higher body mass index, previous ipsilateral DVT, older age, and female sex seem to predict the long term severity of postthrombotic syndrome. ${ }^{2}$ 
Figure 3

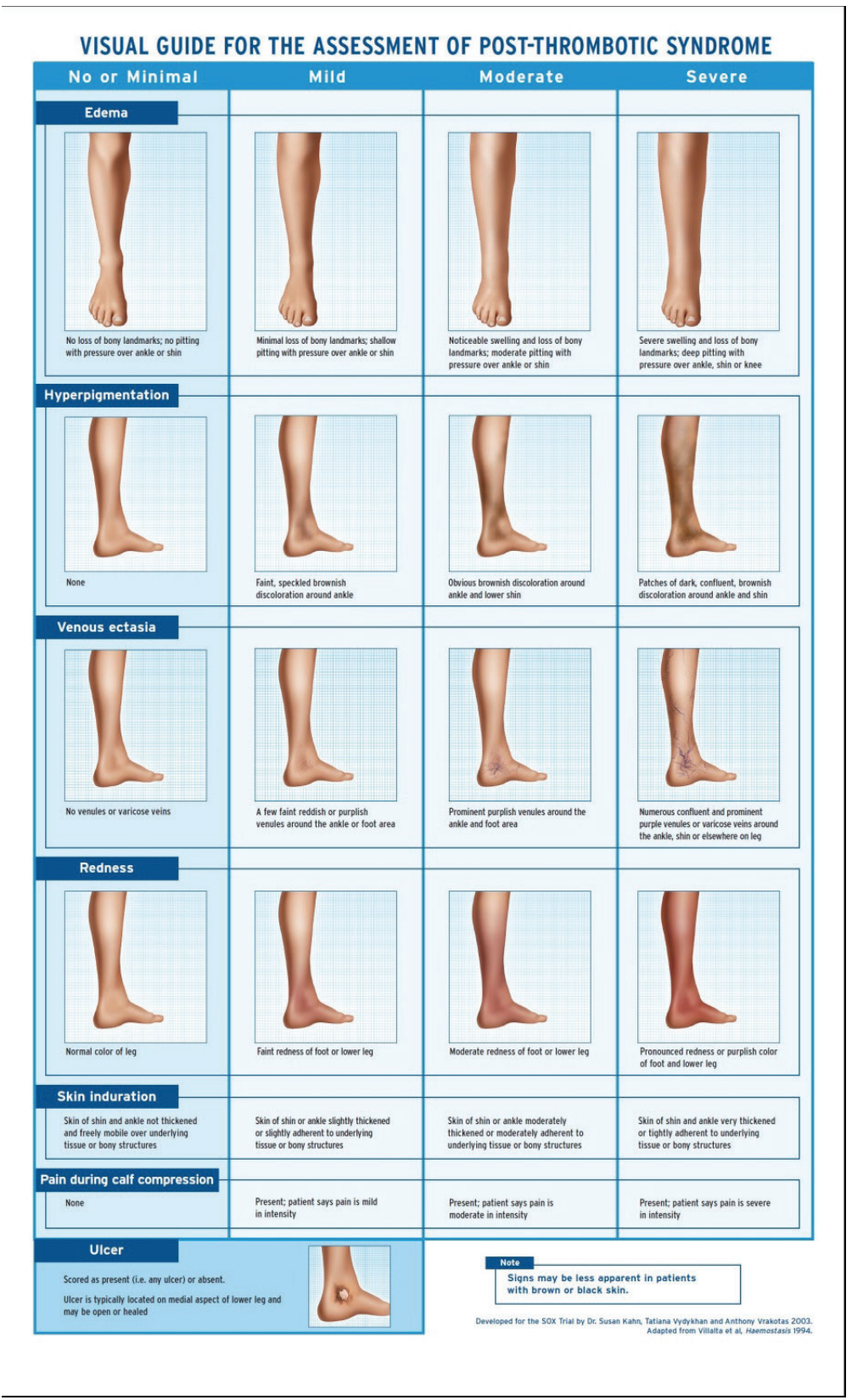

Visual guide to scoring the physician part of the villalta scale. 
Risk of venous ulcer disease

Of those who develop post-thrombotic syndrome, 3-5\% go on to develop venous ulcers, ${ }^{19}$ which are usually painful, resistant to treatment, and tend to recur. Venous ulcers may greatly impair the patient's quality of life and incur high healthcare costs. ${ }^{2}$ Figure 4 illustrates venous ulcer disease in a patient with post-thrombotic syndrome.

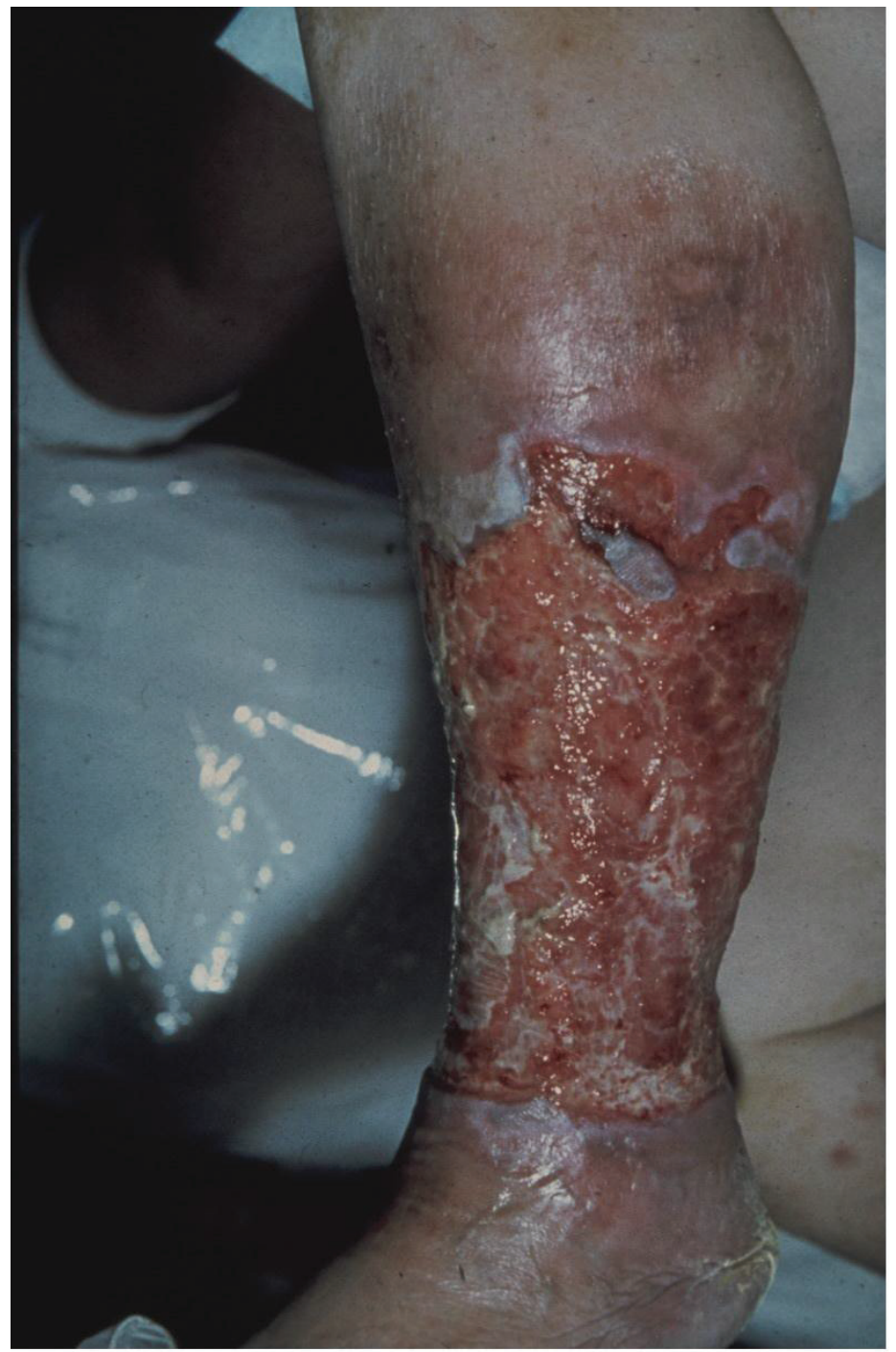

Fig 4 Venous ulceration of the leg. Active ulceration is the most severe form of post-thrombotic syndrome. Dark red pigmentation and painful skin thickening (lipodermatosclerosis) is seen proximal to the ulceration 
What is the current standard therapy for DVT?

Standard treatment for DVT is immediate anticoagulation with subcutaneous low molecular weight heparin and later with oral anticoagulants. Compression treatment with elastic stockings and early ambulation also form part of conservative treatment. ${ }^{20}$ Guidelines from the American College of Chest Physicians (ACCP), which are based on level $1 \mathrm{~A}$ evidence from high quality randomized controlled trials, ${ }^{13}$ recommend immediate anticoagulation with subcutaneous low molecular weight heparins for at least five days, and that the patient should simultaneously take oral anticoagulants such as warfarin for at least three months. The duration of oral treatment depends on the cause of the DVT and whether it can be eliminated or not. Anticoagulation alone decreases the risk of pulmonary embolism to $3.8 \%$, the risk of recurrent DVT to $30 \%$, and the risk of post-thrombotic syndrome to $82 \%$.

ACCP guidelines also recommend that compression treatment be started immediately, using compressive bandaging or elastic stockings with a compression pressure of $30-40 \mathrm{~mm} \mathrm{Hg}$, and continued for at least two years; this is based on evidence from high quality randomized trials. Compression does not reduce the risk of pulmonary embolism or recurrence of DVT, but it has been shown to decrease the incidence of post-thrombotic syndrome by about $50 \%$ at two years. Immediate mobilization is also recommended. Conservative treatment can be safely carried out at home.13,21-23

Although the standard treatment regimen reduces the risk of pulmonary embolism, recurrent DVT, and post-thrombotic syndrome, patients remain at increased risk of pulmonary embolism, recurrence of DVT (30\% within five years), and post-thrombotic syndrome ( $43 \%$ at two years). Patients treated with anticoagulants are also at increased risk of severe bleeding complications. ${ }^{24-26}$ This has led to research into new treatments for DVT.

\section{Are there any new treatments under study for iliofemoral DVT?}

\section{Catheter directed thrombolysis}

A recent observational study found that the degree of clot lysis at treatment is directly correlated with long-term outcome after iliofemoral DVT.27 Patients with iliofemoral DVT have double the risk of recurrent thrombosis compared with those with DVT below this segment. ${ }^{28}$ On the basis of these findings experts consider that patients with iliofemoral DVT might benefit most from a more aggressive approach to thrombus removal.

A systematic review has shown that the administration of systemic thrombolytic agents-aimed at achieving indirect clot lysis-achieves a small increase in 
vein segment patency, but the risk of clinically relevant bleeding complications is high, so systemic thrombolysis is no longer used for DVT. ${ }^{29}$ However, findings from a non-randomized prospective study of catheter directed thrombolysis, which involves infusion of thrombolytic agents through a catheter directly into the thrombus, suggested that the technique can safely lyse thrombus without appreciable systemic effects and may also restore normal valve function and prevented post-thrombotic reflux (a further risk factor for post-thrombotic syndrome). ${ }^{30}$

The findings of several non-randomized studies and two small randomized controlled trials that compared catheter directed thrombolysis with standard anticoagulation treatment show that catheter directed thrombolysis may reduce the incidence of post-thrombotic syndrome compared with standard anticoagulation alone. ${ }^{31-39}$ A systematic review of published studies reported that catheter directed thrombolysis, as an adjunct to standard DVT treatment, reduced the incidence of post-thrombotic syndrome from $78 \%$ to $27 \%$, although the rate of minor bleeding complications was $8 \%$ in patients with thrombolytic treatment. ${ }^{29,31}$ Small retrospective studies of catheter directed thrombolysis for iliofemoral DVT have suggested that successful catheter directed thrombolysis may have a positive effect on the validated health-related quality of life (HRQOL) questionnaire, but further study is needed. . $2,34^{2}$

Currently, three large randomized controlled trials are under way to investigate the effectiveness and safety of catheter directed thrombolysis (the Norwegian CaVent trial, 40 the North American ATTRACT trial, ${ }^{41}$ and the Dutch CAVA trial). Results of these trials, for which the primary outcome is risk of postthrombotic syndrome, are much anticipated. If they robustly show that thrombus removal using chemical and mechanical techniques significantly decreases post-thrombotic syndrome and improves clinical outcomes, the treatment of iliofemoral DVT may be revolutionized.

\section{Combined mechanical and chemical thrombolysis}

Several catheter directed thrombolysis devices now combine mechanical energy with chemical thrombolysis (fig 5), and one of these systems has been shown to be effective in the treatment of peripheral arterial occlusions, ${ }^{35}$ massive pulmonary embolism, ${ }^{42}$ and acute ischemic stroke. ${ }^{43,44}$ Two small patient series have evaluated this ultrasound accelerated catheter directed thrombolysis for the treatment of patients with DVT and shown comparable results. ${ }^{35,36}$

All combined systems require evaluation in randomized studies with large sample sizes because currently there is only low quality evidence to support their use. ${ }^{45}$ The Dutch CAVA trial is evaluating the EKOS Endowave system. ${ }^{46}$ 


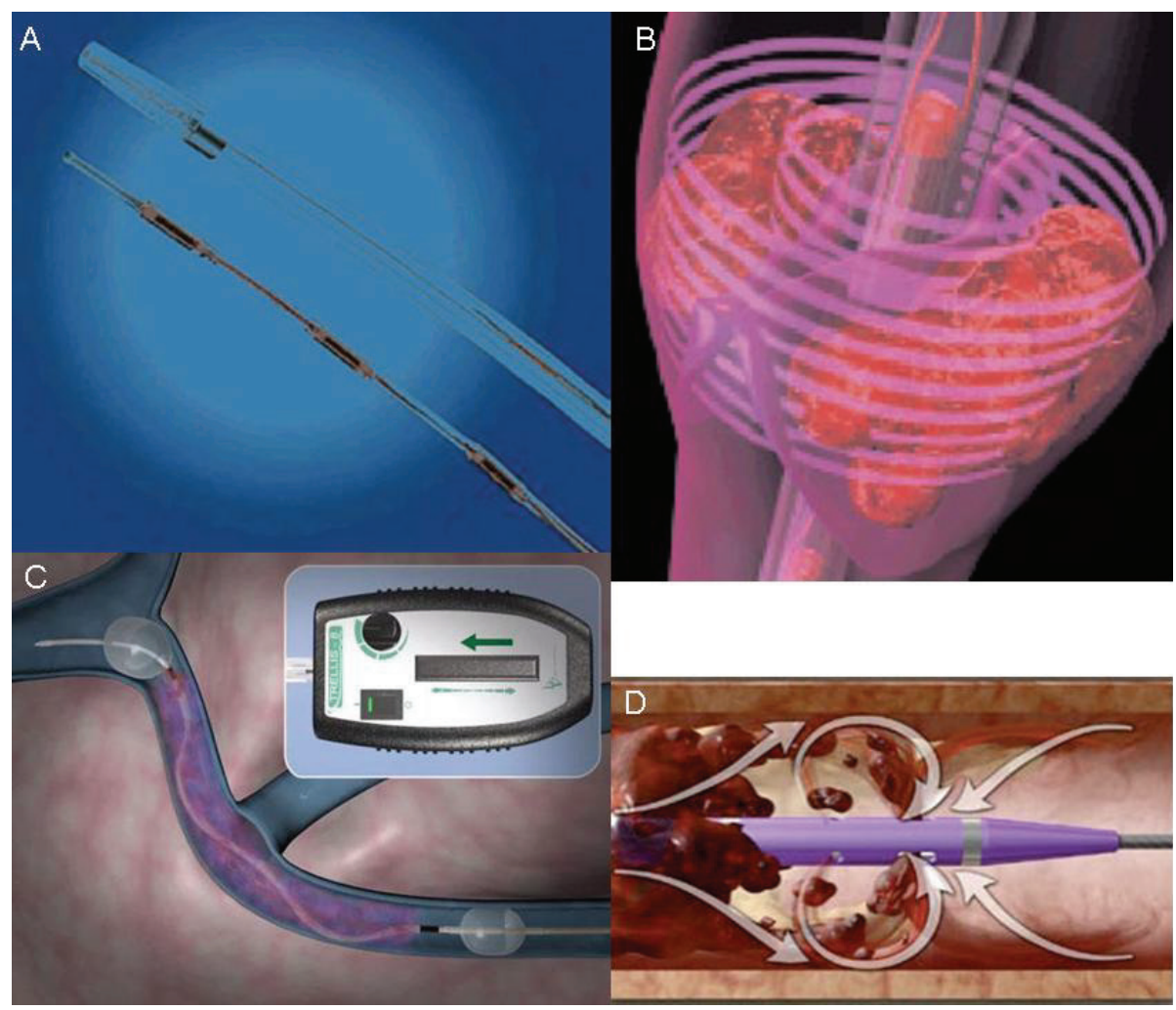

Fig 5. Overview of pharmacomechanical thrombolytic devices. (A and B) The EKOS Endowave Peripheral Lysis System consists of a multi-lumen infusion catheter with removable, coaxial ultrasound core and a control unit that simultaneously delivers high frequency $(2.2 \mathrm{MHz})$, low energy $(0.45 \mathrm{~W})$ ultrasound energy and thrombolytic drug into the thrombus. Ultrasound accelerated catheter directed thrombolysis was developed to overcome the limitations of long treatment times and high drug doses in standard catheter directed thrombolysis. ${ }^{36}$ In vitro, ultrasound energy proved to increase uptake and penetration of the thrombolytic agents into the thrombus by disaggregating the fibrin matrix and exposing additional plasminogen receptor sites to the thrombolytic agent. 47,48 (C) The Trellis-8 catheter directed thrombolysis device combines balloon containment of a thrombus with chemical and mechanical thrombolysis. The thrombus is isolated after placing the catheter by inflating the proximal and distal balloons. The thrombolytic drug is then infused through the catheter and exits through multiple side holes in between the balloons. This is followed by removal of the guide wire and placement of a stiff sinusoidal wire between the balloons. A battery powered motor then turns the wire at 3000 rpm; thus, mechanical dissolution of the thrombus is combined with the actions of the thrombolytic drug. ${ }^{49}$ (D) The Angiojet Power Pulse system uses a com- 
plex mixture of rapid fluid streaming and hydrodynamic forces to fracture the thrombus, allowing extraction at the catheter tip as a result of negative pressure. The catheter infuses normal saline through an infusion port while simultaneously suctioning through the effluent port. If the effluent port is clamped, the infusion port acts as a mechanical "pulse spray" that delivers the preloaded thrombolytic drug to the thrombus. ${ }^{45,50}$ 


\section{References:}

1. Nordstrom M, Lindblad B, Bergqvist D, Kjellstrom T. A prospective study of the incidence of deep-vein thrombosis within a defined urban population. J Intern Med 1992 Aug;232(2):15560.

2. Kahn SR, Shrier I, Julian JA, Ducruet T, Arsenault L, Miron MJ, et al. Determinants and time course of the postthrombotic syndrome after acute deep venous thrombosis. Ann Intern Med 2008;149):698-707.

3. Lensing AW, Prandoni P, Prins MH, Buller HR. Deep-vein thrombosis. Lancet 1999;353:479-85.

4. Prandoni P, Noventa F, Ghirarduzzi A, Pengo V, Bernardi E, Pesavento R, et al. The risk of recurrent venous thromboembolism after discontinuing anticoagulation in patients with acute proximal deep vein thrombosis or pulmonary embolism. A prospective cohort study in 1626 patients. Haematologica 2007;92:199-205.

5. Kahn SR, Shbaklo H, Lamping DL, Holcroft CA, Shrier I, Miron MJ, et al. Determinants of healthrelated quality of life during the 2 years following deep vein thrombosis. J Thromb Haemost 2008;6:1105-12.

6. Bulger CM, Jacobs C, Patel NH. Epidemiology of acute deep vein thrombosis. Tech Vasc Interv Radiol 2004;7:50-4.

7. Wells PS, Anderson DR, Rodger M, Forgie M, Kearon C, Dreyer J, et al. Evaluation of D-dimer in the diagnosis of suspected deep-vein thrombosis. N Engl J Med 2003;349:1227-35.

8. Linkins LA, Bates SM, Ginsberg JS, Kearon C. Use of different D-dimer levels to exclude venous thromboembolism depending on clinical pretest probability. J Thromb Haemost 2004;2:125660.

9. Van der Velde EF, Toll DB, Ten Cate-Hoek AJ, Oudega R, Stoffers HE, Bossuyt PM, et al. Comparing the diagnostic performance of 2 clinical decision rules to rule out deep vein thrombosis in primary care patients. Ann Fam Med 2011;9:31-6.

10. Miller N, Satin R, Tousignant L, Sheiner NM. A prospective study comparing duplex scan and venography for diagnosis of lower-extremity deep vein thrombosis. Cardiovasc Surg 1996;4:505-8.

11. van Langevelde K, Tan M, Sramek A, Huisman MV, de Roos A. Magnetic resonance imaging and computed tomography developments in imaging of venous thromboembolism. J Magn Reson Imaging 2010;32:1302-12.

12. Baldt MM, Zontsich T, Stumpflen A, Fleischmann D, Schneider B, Minar E, et al. Deep venous thrombosis of the lower extremity: efficacy of spiral CT venography compared with conventional venography in diagnosis. Radiology 1996;200:423-8.

13. Kearon C, Kahn SR, Agnelli G, Goldhaber S, Raskob GE, Comerota AJ. Antithrombotic therapy for venous thromboembolic disease: American College of Chest Physicians evidence-based clinical practice guidelines (8th edition). Chest 2008;133(6 suppl):454S-545S.

14. Buller HR, Agnelli G, Hull RD, Hyers TM, Prins MH, Raskob GE. Antithrombotic therapy for venous thromboembolic disease: the Seventh ACCP Conference on Antithrombotic and Thrombolytic Therapy. Chest 2004;126(3 suppl):401S-28S.

15. Kearon C. Natural history of venous thromboembolism. Circulation 2003;107(23 suppl 1):I2230.

16. Kahn SR, Ginsberg JS. Relationship between deep venous thrombosis and the postthrombotic syndrome. Arch Intern Med 2004;164:17-26.

17. Kahn SR, Partsch H, Vedantham S, Prandoni P, Kearon C. Definition of post-thrombotic syndrome of the leg for use in clinical investigations: a recommendation for standardization. J Thromb Haemost 2009;7:879-83.

18. Singh H, Masuda EM. Comparing short-term outcomes of femoral-popliteal and iliofemoral deep venous thrombosis: early lysis and development of reflux. Ann Vasc Surg 2005;19:74-9.

19. Van Gent WB, Wilschut ED, Wittens C. Management of venous ulcer disease. BMJ 2010;341:c6045. 
20. Hirsh J, Guyatt G, Albers GW, Harrington R, Schunemann HJ. Executive summary: American College of Chest Physicians evidence-based clinical practice guidelines (8th edition). Chest 2008;133(6 suppl):71S-109S.

21. Blattler W, Partsch H. Leg compression and ambulation is better than bed rest for the treatment of acute deep venous thrombosis. Int Angiol 2003;22:393-400.

22. Othieno $R$, Abu Affan $M$, Okpo E. Home versus in-patient treatment for deep vein thrombosis. Cochrane Database Syst Rev 2007;3:CD003076.

23. Prandoni P, Lensing AW, Prins MH, Frulla M, Marchiori A, Bernardi E, et al. Below-knee elastic compression stockings to prevent the post-thrombotic syndrome: a randomized, controlled trial. Ann Intern Med 2004;141:249-56.

24. Prandoni P, Lensing AW, Cogo A, Cuppini S, Villalta S, Carta M, et al. The long-term clinical course of acute deep venous thrombosis. Ann Intern Med 1996;125:1-7.

25. Kearon C, Gent M, Hirsh J, Weitz J, Kovacs MJ, Anderson DR, et al. A comparison of three months of anticoagulation with extended anticoagulation for a first episode of idiopathic venous thromboembolism. N Engl J Med 1999;340:901-7.

26. Douketis JD, Kearon C, Bates S, Duku EK, Ginsberg JS. Risk of fatal pulmonary embolism in patients with treated venous thromboembolism. JAMA 1998;279:458-62.

27. Grewal NK, Martinez JT, Andrews L, Comerota AJ. Quantity of clot lysed after catheter-directed thrombolysis for iliofemoral deep venous thrombosis correlates with postthrombotic morbidity. J Vasc Surg 2010;51:1209-14.

28. Douketis JD, Crowther MA, Foster GA, Ginsberg JS. Does the location of thrombosis determine the risk of disease recurrence in patients with proximal deep vein thrombosis? Am J Med 2001;110:515-9.

29. Alesh I, Kayali F, Stein PD. Catheter-directed thrombolysis (intrathrombus injection) in treatment of deep venous thrombosis: a systematic review. Catheter Cardiovasc Interv 2007;70:143-8.

30. Meissner MH, Manzo RA, Bergelin RO, Markel A, Strandness DE Jr. Deep venous insufficiency: the relationship between lysis and subsequent reflux. J Vasc Surg 1993;18:596-605; discussion 6-8.

31. Mewissen MW, Seabrook GR, Meissner MH, Cynamon J, Labropoulos N, Haughton SH. Catheterdirected thrombolysis for lower extremity deep venous thrombosis: report of a national multicenter registry. Radiology. 1999 Apr;211(1):39-49.

32. Comerota AJ, Throm RC, Mathias SD, Haughton S, Mewissen M. Catheter-directed thrombolysis for iliofemoral deep venous thrombosis improves health-related quality of life. J Vasc Surg. 2000 Jul;32(1):130-7.

33. Mewissen MW. Catheter-directed thrombolysis for lower extremity deep vein thrombosis. Tech Vasc Interv Radiol. 2001 Jun;4(2):111-4.

34. Comerota AJ. Quality-of-life improvement using thrombolytic therapy for iliofemoral deep venous thrombosis. Rev Cardiovasc Med. 2002;3 Suppl 2:S61-7.

35. Motarjeme A. Ultrasound-enhanced Thrombolysis. J Endovasc Ther. 2007 Apr;14(2):251-6.

36. Parikh S, Motarjeme A, McNamara T, Raabe R, Hagspiel K, Benenati JF, et al. Ultrasoundaccelerated thrombolysis for the treatment of deep vein thrombosis: initial clinical experience. J Vasc Interv Radiol. 2008 Apr;19(4):521-8.

37. Baekgaard N, Broholm R, Just S, Jorgensen M, Jensen LP. Long-term results using catheterdirected thrombolysis in 103 lower limbs with acute iliofemoral venous thrombosis. Eur J Vasc Endovasc Surg. Jan;39(1):112-7.

38. Elsharawy M, Elzayat E. Early results of thrombolysis vs anticoagulation in iliofemoral venous thrombosis. A randomised clinical trial. Eur J Vasc Endovasc Surg. 2002 Sep;24(3):209-14.

39. Enden T, Klow NE, Sandvik L, Slagsvold CE, Ghanima W, Hafsahl G, et al. Catheter-directed thrombolysis vs. anticoagulant therapy alone in deep vein thrombosis: results of an open randomized, controlled trial reporting on short-term patency. J Thromb Haemost. 2009 Aug;7(8):1268-75. 
40. Enden T, Sandvik L, Klow NE, Hafsahl G, Holme PA, Holmen LO, et al. Catheter-directed Venous Thrombolysis in acute iliofemoral vein thrombosis-the CaVenT study: rationale and design of a multicenter, randomized, controlled, clinical trial (NCT00251771). Am Heart J. 2007 Nov;154(5):808-14.

41. Comerota AJ. The ATTRACT Trial: Rationale for Early Intervention for Iliofemoral DVT. Perspect Vasc Surg Endovasc Ther. Jan 3.

42. Chamsuddin A, Nazzal L, Kang B, Best I, Peters G, Panah S, et al. Catheter-directed thrombolysis with the Endowave system in the treatment of acute massive pulmonary embolism: a retrospective multicenter case series. J Vasc Interv Radiol. 2008 Mar;19(3):372-6.

43. The Interventional Management of Stroke (IMS) II Study. Stroke. 2007 Jul;38(7):2127-35.

44. Tsivgoulis G, Eggers J, Ribo M, Perren F, Saqqur M, Rubiera M, et al. Safety and efficacy of ultrasound-enhanced thrombolysis: a comprehensive review and meta-analysis of randomized and nonrandomized studies. Stroke. Feb;41(2):280-7.

45. Grommes J, Strijkers R, Greiner A, Mahnken AH, Wittens CH. Safety and feasibility of ultrasound-accelerated catheter-directed thrombolysis in deep vein thrombosis. Eur J Vasc Endovasc Surg. 2011 Apr;41(4):526-32.

46. O’Sullivan GJ, Lohan DG, Gough N, Cronin CG, Kee ST. Pharmacomechanical thrombectomy of acute deep vein thrombosis with the Trellis-8 isolated thrombolysis catheter. J Vasc Interv Radiol. 2007 Jun;18(6):715-24.

47. Martinez Trabal JL, Comerota AJ, LaPorte FB, Kazanjian S, DiSalle R, Sepanski DM. The quantitative benefit of isolated, segmental, pharmacomechanical thrombolysis (ISPMT) for iliofemoral venous thrombosis. J Vasc Surg. 2008 Dec;48(6):1532-7.

48. Hilleman DE, Razavi MK. Clinical and economic evaluation of the Trellis-8 infusion catheter for deep vein thrombosis. J Vasc Interv Radiol. 2008 Mar;19(3):377-83.

49. Lin PH, Ochoa LN, Duffy P. Catheter-directed thrombectomy and thrombolysis for symptomatic lower-extremity deep vein thrombosis: review of current interventional treatment strategies. Perspect Vasc Surg Endovasc Ther. 2010 Sep;22(3):152-63.

50. Lin PH, Zhou W, Dardik A, Mussa F, Kougias P, Hedayati N, et al. Catheter-direct thrombolysis versus pharmacomechanical thrombectomy for treatment of symptomatic lower extremity deep venous thrombosis. Am J Surg. 2006 Dec;192(6):782-8.

51. Rao AS, Konig G, Leers SA, Cho J, Rhee RY, Makaroun MS, et al. Pharmacomechanical thrombectomy for iliofemoral deep vein thrombosis: an alternative in patients with contraindications to thrombolysis. J Vasc Surg. 2009 Nov;50(5):1092-8.

52. Francis CW, Blinc A, Lee S, Cox C. Ultrasound accelerates transport of recombinant tissue plasminogen activator into clots. Ultrasound Med Biol. 1995;21(3):419-24.

53. Braaten JV, Goss RA, Francis CW. Ultrasound reversibly disaggregates fibrin fibers. Thromb Haemost. 1997 Sep;78(3):1063-8.

54. McLafferty RB. Endovascular management of deep venous thrombosis. Perspect Vasc Surg Endovasc Ther. 2008 Mar;20(1):87-91. 


\section{CHAPTER 3 \\ Villalta scale: Goals and limitations}

Rob H.W. Strijkers

Cees H.A. Wittens

Susan R. Kahn

Phlebology march 2012 vol. 27, suppl 1: pp. 130-135 


\begin{abstract}
The post-thrombotic syndrome (PTS) is a common, debilitating complication following deep venous thrombosis. PTS is a syndrome for which it has been difficult to provide a clear definition. PTS was defined as chronic venous signs/symptoms after a deep venous thrombosis. A number of scales are available to diagnose PTS, but there is a strong need for standardization. The Villalta scale was chosen as the standardized PTS scale. The Villalta scale has been validated in several studies and shows good correlation with generic and disease-specific quality of life scales. The inter-observer rate between study nurses and physicians was shown to be very good. Furthermore, the scale shows good discriminative properties among the different severity groups of PTS. The Villalta scale is useful for use in randomized controlled trials and it allows pooling of data from different studies. The Villalta scale should undergo further validation on intra-rater reliability. Ongoing randomized controlled trials will further validate the Villalta scale for use in PTS.
\end{abstract}

Keywords: post-thrombotic syndrome; PTS; Villalta scale; validation 


\section{Introduction}

Post thrombotic syndrome (PTS) is the most common complication following deep venous thrombosis. ${ }^{1}$ PTS is a chronic, sometimes debilitating disease characterized by a range of clinical symptoms and patient complaints. Estimates show that $20-50 \%$ of patients will develop PTS within the first 2 years after a DVT. Quality of life is diminished in patients with PTS and that quality of life is comparable to patients with chronic diseases such as diabetes and chronic heart failure. ${ }^{2}$ After a DVT 3-5\% of all patients will go on to develop a venous ulcer. ${ }^{3}$ Patients with venous ulceration have poor quality of life and have high healthcare costs. ${ }^{4}$ In the United States, 200 million dollars per year is spent on the treatment of venous stasis disease. ${ }^{5}$ Until recently, research on the treatment of DVT has primarily focused on short term complications of DVT such as recurrence of DVT, bleeding risk and pulmonary embolism and less on PTS. Recent studies focus on the long-term results of DVT treatment and especially on PTS. However, a challenge has been how to define PTS. Standardization of the definition of PTS makes it possible to create and test scales to identify PTS and assess its severity. A number of proposed PTS scores are available and it is difficult to compare and combine research results using different scales. There is a strong need for standardization of PTS scales to ensure high quality research with validated endpoints and good reproducibility.

\section{Clinical presentation of PTS}

PTS consists of both patient symptoms and clinical signs. Symptoms include pain, heaviness, swelling, itching, cramps or tingling in the affected leg. These can vary from patient to patient and all combinations of symptoms are possible. Symptoms can be persistent or intermittent. Exercise tends to aggravate symptoms and rest and elevation of the leg reduces symptoms. Clinical signs include telangiectasia, eczema, varicose veins, edema, lipodermatosclerosis and ulceration $^{7-10 .}$

\section{Definition of PTS}

Because of the wide range of clinical presentations, it has proven difficult to achieve consensus on a clear definition of PTS. PTS is a syndrome and there is no gold standard diagnostic test. An ad-hoc conference organized by the European Venous Forum defined PTS as chronic venous symptoms/signs secondary to deep venous thrombosis. ${ }^{6}$ In the past 20 years a few scales have been introduced to help assess whether a patient has PTS or not. PTS is considered when a patient has had a deep venous thrombosis and has complaints in the ipsilateral leg11 that have developed or persisted a few months after DVT ${ }^{12}$. Ideally, a PTS scale should assess whether PTS is present or not and preferably distinguish between severe and milder forms of PTS. The scale should be easy to use 
with high inter- and intra-observer rates so that scores are reproducible and the scale can be used for clinical research and daily practice. The scale should be accepted and used worldwide, so that pooling of results is possible and the strongest evidence becomes available.

\section{Available PTS Scales}

Different scoring systems have been proposed to diagnose PTS. These include the VCSS, CEAP classification, Widmer classification, PTS scoring system according to Brandjes, Ginsberg scale, and Villalta scale. The CEAP and VCSS are specific for venous disease, but not designed for PTS. The CEAP classification only includes clinical signs and does not include patient complaints. ${ }^{13}$ The VCSS was developed to give a quantifiable system with gradable elements that could change in response to treatment.14 The Widmer classification was meant to grade chronic venous insufficiency and is also not specific for PTS. Widmer used purely the objective clinical signs to assess the severity of PTS in patients. ${ }^{17}$ The Brandjes, Ginsberg, and Villalta scale are specific scores to assess PTS. Table 1 provides an overview of the different scales and their properties.

Table 1: Available PTS scales

\begin{tabular}{l|l|l|l|}
\hline Scales & Clinical signs included? & Patiënt symptoms included? & Specific for PTS \\
\hline CEAP & Yes & No & No \\
VCSS & Yes & Yes & No \\
Widmer & Yes & No & No \\
\hline Brandjes & Yes & Yes & Yes \\
Ginsberg & Yes & Yes & Yes \\
Villalta & Yes & Yes & Yes
\end{tabular}

Brandjes developed a scoring system for PTS, which characterizes patients into three, groups (no PTS, mild-to-moderate PTS and severe PTS) using objective clinical signs and patient symptoms. All symptoms are scored as either present or absent. ${ }^{18}$ Ginsberg used clinical signs and symptoms to identify the presence of PTS, but did not distinguish between mild-to-moderate or severe PTS. ${ }^{19}$ This limits the Ginsberg score for research purposes. ${ }^{20}$ The Villalta scale takes clinical signs and patient symptoms into account, and also classifies patients into different groups according to PTS severity. ${ }^{21}$ Tables 2 and 3 show the Brandjes scale and the Villalta scale. 
Table 2

\begin{tabular}{|l|l|l|l|l|}
\hline PTS severity & Subjective criteria & & Objective criteria \\
\hline Mild-to-moderate PTS (score 3)* & symptom & spontaneous pain in calf & sign \\
\hline & Spontaneous pain in thigh & 1 & Calf circumference increased by $1 \mathrm{~cm}$ \\
\hline & pain in calf on standing/walking & 1 & Ankle circumference increased by $1 \mathrm{~cm}$ \\
\hline pain in thigh on standing/walking & 1 & Pigmentation \\
\hline Edema on foot/calf & 1 & Venectasia \\
\hline Heaviness of leg & 1 & Newly formed varicosis \\
\hline Spontaneous pain and pain on standing/walking & 1 & Calf circumference increased by $1 \mathrm{~cm}$ \\
\hline & Edema of calf & 1 & 1 \\
\hline & Imparement of daily activities & 1 & Pigmentation, discolouration, and venectasia & 1 \\
\hline
\end{tabular}

Table 3

\begin{tabular}{|l|l|l|l|l|}
\hline $\begin{array}{l}\text { Symptoms and clinical } \\
\text { signs }\end{array}$ & None & Mild & Moderate & Severe \\
\hline Symptoms & 0 & 1 & 2 & 3 \\
\hline $\begin{array}{l}\text { Pain } \\
\text { cramps }\end{array}$ & 0 & 1 & 2 & 3 \\
\hline Heaviness & 0 & 1 & 2 & 3 \\
\hline Paresthesia & 0 & 1 & 2 & 3 \\
\hline Pruritis & 0 & 1 & 2 & 3 \\
\hline Clinical signs & & & & \\
\hline Pretibial edema & 0 & 1 & 2 & 3 \\
\hline Skin induration & 0 & 1 & 2 & 3 \\
\hline Hyperpigmentation & 0 & 1 & 2 & 3 \\
\hline Redness & 0 & 1 & 2 & 3 \\
\hline Venous ectasia & 0 & 1 & 2 & 3 \\
\hline Pain on calf compression & 0 & 1 & 2 & 3 \\
\hline Venous ulcer & absent & present & & \\
\hline
\end{tabular}

\section{Villalta scale}

Recently, the subcommittee on control of Anticoagulation of the Scientific and Standardization Committee of the International Society on Thrombosis and Haemostasis recommended that the Villalta Scale should be adopted in clinical studies to diagnose and grade the severity of PTS.11 The committee reviewed the scales mentioned above and concluded that the Villalta scale met most of the criteria which a standard scale should consist of. The specific reasons for recommending the Villalta scale were: the use of both clinical signs and subjective symptoms with severity and range taken into account, demonstrated validity, good inter-rater reliability, ease to use, good nurse-physician reliability and 
the advantage of being able to use the scale in multiple ways (binary, categorical or continuous) and also fact that it has been successfully used in clinical trials. ${ }^{22}$

The Villalta scale is a validated score for assessing PTS. ${ }^{20}$ The scale consists of subjective symptoms reported by the patient and the second part of the scale consists of objective signs observed by the clinician. Together the scale consists of 11 elements that are scored from 0 to 3, with 0 being absent, 1 meaning mild, 2 meaning moderate and 3 meaning severe. Table 3 shows all the elements in the Villalta score. Figure 1 is a visual guide for the 6 signs of the Villalta score. Leg ulceration is also present in the scale, but is only scored as either present or absent. Scores of $<5$ indicate absence of PTS, a score 5-9 indicates mild PTS, 10-14 indicates moderate PTS, and a Villalta score of $>15$ or ulceration indicates severe PTS. Because PTS can develop anywhere in the years following a DVT it is important to keep track of progression. During each follow-up, PTS can be assessed using the Villalta scale. Some previous studies do not differentiate between mild and moderate PTS, but define mild/moderate PTS as a Villalta score of $5-14.3,24,25$

\section{Validation of the Villalta scale}

Validation of the Villalta scale is difficult, because there is no comparison possible to a gold standard.11 Comparison is only possible to known anatomical/physiological changes correlated with PTS, disease specific quality of life scores and generic quality of life scores.

The Villalta scale has shown evidence of validity in several studies. PTS severity and patients' perception of disease burden correlated with quality of life scores (generic quality of life (SF-36) and the venous disease specific quality of life (VEINES-QOL). ${ }^{26}$ Research showed that patients one year after DVT who had PTS according to the Villalta scale had significantly worse health related quality of life scores. ${ }^{2,20,25,27,28}$ The Villalta scale shows good reproducibility and good correlation with the patients perception of interference with daily life. ${ }^{25,29} \mathrm{~A}$ substudy of the REVERSE study assessed the inter-rater reliability of the Villalta scale. ${ }^{30}$ Inter-rater reliability was shown to be very good between study nurses and physicians. The study nurses received one hour of training before assessing the clinical signs of the patient. The inter-rater reliability for the cut off no PTS vs. PTS was good ( $\mathrm{K}=0.71)$ and for moderate/severe PTS vs. No PTS was excellent $(\mathrm{K}=1.00){ }^{31}$ To our knowledge, test-retest validation has not yet been performed in the literature. This would be relevant to assess for the patient-completed parts of the Villalta scale. ${ }^{11}$ The Villalta scale showed good discriminative properties among the different severity groups. ${ }^{29}$ 


\section{Use of PTS scales in ongoing research}

Until recently, DVT research focused on short term endpoints like DVT recurrence, pulmonary embolism and bleeding risk. Lately, there has been added focus on long-term follow-up of patients after DVT and prevalence of PTS, especially after iliofemoral DVT. Three current ongoing randomized controlled trials are all using the Villalta scale for assessment of PTS at specific time points: the CaVenT-study, Attract trial and CAVA-trial. ${ }^{32-34}$ The primary endpoint of these trials is reduction of PTS at one or two years follow-up. The rationale for a follow-up period of one year or longer is that PTS tends to become evident in the first 1-2 years, hence a shorter follow-up time will miss cases of PTS 35.

\section{Limitations of the Villalta scale}

Although validity of the Villalta scale has been tested in research, there are still aspects of the scale, which have not been validated. Future research could focus on further evaluating its validity and reliability, especially its intra-rater reliability. Administration of the scale requires training and familiarity with assessment of various signs of venous disease. The scale may be somewhat nonspecific as some of the symptoms and signs it assesses are not specific to venous disease.

\section{Conclusion}

PTS is a chronic burdensome disease that affects a great number of patients after DVT. As healthcare costs associated with PTS are high, research is needed on approaches aimed to lower the incidence of PTS. To be able to produce the best evidence for PTS prevention and treatment, standardization of the measurement of PTS is needed. While a number of scales exist, the Villalta scale, due to its properties, may be considered a very good choice. Ongoing major trials of PTS prevention will report on the incidence of PTS by means of the Villalta scale. 
Figure 1: developed by Dr. S. Kahn and used with permission VISUAL GUIDE FOR THE ASSESSMENT OF POST-THROMBOTIC SYNDROME

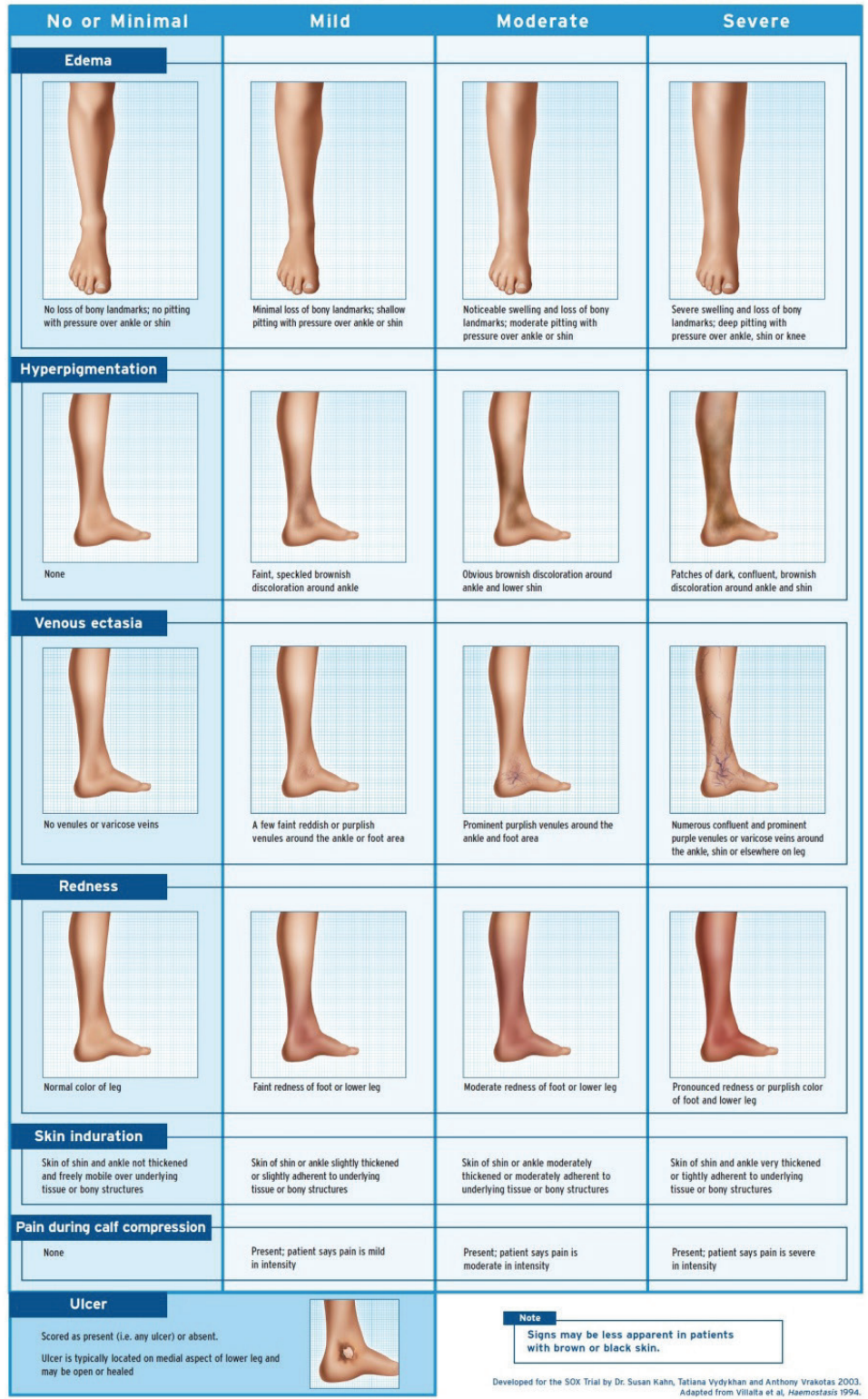




\section{References:}

1. Ashrani AA, Heit JA. Incidence and cost burden of post-thrombotic syndrome. J Thromb Thrombolysis. 2009;28(4):465-476.

2. Kahn Sr, Shbaklo H, Lamping DL, et al. Determinants of health-related quality of life during the 2 years following deep vein thrombosis. Journal of Thrombosis and Haemostasis. 2008;6(7):1105-1112.

3. Prandoni P, Lensing AWA, Cogo A, et al. The long-term clinical course of acute deep venous thrombosis. Ann. Intern. Med. 1996;125(1):1.

4. Olin JW, Beusterien KM, Childs MB, et al. Medical costs of treating venous stasis ulcers: evidence from a retrospective cohort study. Vasc Med. 1999;4(1):1-7.

5. Heit JA, Rooke TW, Silverstein MD, et al. Trends in the incidence of venous stasis syndrome and venous ulcer: a 25-year population-based study. YMVA. 2001;33(5):1022-1027.

6. Eklof B, Perrin M, Delis KT, et al. Updated terminology of chronic venous disorders: the VEINTERM transatlantic interdisciplinary consensus document. J. Vasc. Surg. 2009;49(2):498-501.

7. Kahn SR, Ginsberg JS. Relationship between deep venous thrombosis and the postthrombotic syndrome. Arch. Intern. Med. 2004;164(1):17-26.

8. Kurz X, Kahn SR, Abenhaim L, et al. Chronic venous disorders of the leg: epidemiology, outcomes, diagnosis and management. Summary of an evidence-based report of the VEINES task force. Venous Insufficiency Epidemiologic and Economic Studies. In: International angiology : a journal of the International Union of Angiology.Vol 18. 1999:83-102.

9. Bernardi E, Prandoni P. The post-thrombotic syndrome. Curr Opin Pulm Med. 2000;6(4):335342.

10. Hopkins N. ABC of vascular diseases. Deep venous insufficiency and occlusion. | BMJ. British Medical Journal. 1992.

11. Kahn Sr, Partsch H, Vedantham S, et al. Definition of post-thrombotic syndrome of the leg for use in clinical investigations: a recommendation for standardization. Journal of Thrombosis and Haemostasis. 2009;7(5):879-883.

12. Kahn SR. The post-thrombotic syndrome: the forgotten morbidity of deep venous thrombosis. J Thromb Thrombolysis. 2006;21(1):41-48.

13. Porter JM, Moneta GL. Reporting standards in venous disease: an update. International Consensus Committee on Chronic Venous Disease. YMVA. 1995;21(4):635-645.

14. Rutherford RB, Padberg FT, Comerota AJ, et al. Venous severity scoring: An adjunct to venous outcome assessment. YMVA. 2000;31(6):1307-1312.

15. Kahn SR, Ducruet T, Lamping DL, et al. Prospective evaluation of health-related quality of life in patients with deep venous thrombosis. Arch. Intern. Med. 2005;165(10):1173-1178.

16. Vedantham S. Definition of postthrombotic disease. YMVA. 2010;52(5):58S-62S.

17. Widmer L, Stahelin H, Amacher A, Brubacher G. Venen-, Arterien-Krankheiten, Koronare Herzkrankheit bei Berufstätigen: Prospektiv-epidemiologische Untersuchung: Basler Studie IIII:[1959-1978]. 1981.

18. Brandjes DP, Büller HR, Heijboer H, et al. Randomised trial of effect of compression stockings in patients with symptomatic proximal-vein thrombosis. The Lancet. 1997;349(9054):759-762.

19. Ginsberg JS, Hirsh J, Julian J, et al. Prevention and treatment of postphlebitic syndrome: results of a 3-part study. Arch. Intern. Med. 2001;161(17):2105-2109.

20. Kahn Sr, Desmarais S, Ducruet T, Arsenault L, Ginsberg JS. Comparison of the Villalta and Ginsberg clinical scales to diagnose the post-thrombotic syndrome: correlation with patientreported disease burden and venous valvular reflux. J. Thromb. Haemost. 2006;4(4):907-908.

21. Kahn SR. Measurement properties of the Villalta scale to define and classify the severity of the post-thrombotic syndrome. Journal of Thrombosis and Haemostasis. 2009;7(5):884-888.

22. van Dongen CJJ, Prandoni P, Frulla M, et al. Relation between quality of anticoagulant treatment and the development of the postthrombotic syndrome. J. Thromb. Haemost. 2005;3(5):939942. 
23. Kahn SR, Shrier I, Julian JA, et al. Determinants and time course of the postthrombotic syndrome after acute deep venous thrombosis. Ann. Intern. Med. 2008;149(10):698-707.

24. Prandoni $P$, Lensing AWA, Prins MH, et al. Below-knee elastic compression stockings to prevent the post-thrombotic syndrome: a randomized, controlled trial. Ann. Intern. Med. 2004;141(4):249-256.

25. Kahn SR, Hirsch A, Shrier I. Effect of postthrombotic syndrome on health-related quality of life after deep venous thrombosis. Arch. Intern. Med. 2002;162(10):1144-1148.

26. Kahn SR, Shrier I, Shapiro S, et al. Six-month exercise training program to treat post-thrombotic syndrome: a randomized controlled two-centre trial. CMAJ. 2011;183(1):37-44.

27. Kahn SR, Lamping DL, Ducruet T, et al. VEINES-QOL/Sym questionnaire was a reliable and valid disease-specific quality of life measure for deep venous thrombosis. Journal of Clinical Epidemiology. 2006;59(10):1056.e1-1056.e4.

28. Kahn Sr, Kearon C, Julian Ja, et al. Predictors of the post-thrombotic syndrome during long-term treatment of proximal deep vein thrombosis. J. Thromb. Haemost. 2005;3(4):718-723.

29. Villalta S, Bagatella P, Piccioli A, et al. Assessment of validity and reproducibility of a clinical scale for the post-thrombotic syndrome. 1994;24(158a).

30. Rodger MA, Kahn SR, Wells PS, et al. Identifying unprovoked thromboembolism patients at low risk for recurrence who can discontinue anticoagulant therapy. CMAJ. 2008;179(5):417-426.

31. Rodger MA, Kahn SR, Le Gal G, et al. Inter-observer reliability of measures to assess the postthrombotic syndrome. Thromb Haemost. 2008;100(1):164-166.

32. Enden T, Sandvik L, Klow N, et al. Catheter-directed Venous Thrombolysis in acute iliofemoral vein thrombosis-the CaVenT Study: Rationale and design of a multicenter, randomized, controlled, clinical trial (NCT00251771). American Heart Journal. 2007;154(5):808-814.

33. Comerota AJ. The ATTRACT Trial: Rationale for Early Intervention for Iliofemoral DVT. Perspectives in Vascular Surgery and Endovascular Therapy. 2010;21(4):221-225.

34. Grommes J, Strijkers RHW, Greiner A, Mahnken AH, Wittens CHA. Safety and feasibility of ultrasound-accelerated catheter-directed thrombolysis in deep vein thrombosis. Eur J Vasc Endovasc Surg. 2011;41(4):526-532.

35. Prandoni P, Villalta S, Bagatella P, et al. The clinical course of deep-vein thrombosis. Prospective long-term follow-up of 528 symptomatic patients. Haematologica. 1997;82(4):423-428. 


\section{CHAPTER 4 \\ Validation of the LET classification}

Rob H.W. Strijkers

Carsten W.K.P. Arnoldussen

Cees H.A. Wittens

Phlebology March 2015 vol. 30 no. 1 suppl 14-19 


\begin{abstract}
Introduction: Acute thrombus removal therapies may reduce post-thrombotic syndrome in certain deep venous thrombosis patients. The LET classification is designed to identify patients at high risk for developing post-thrombotic syndrome in the acute phase using thrombus location and extent. This study evaluates the use of the LET classification to predict post-thrombotic syndrome in a cohort of patients after deep venous thrombosis.
\end{abstract}

Methods: A cohort of 660 deep venous thrombosis patients was invited to participate and fill out a questionnaire with Quality of life questionnaires, basic information, a modified self-assessment version of the Villalta scale for postthrombotic syndrome and potential confounders. Original data on thrombus extension was available, and the LET classes were determined. Obtained information was analysed using ANOVA and in a multiple logistic regression model to correct for confounders. All different LET classes were compared to postthrombotic syndrome occurrence, post-thrombotic syndrome prediction and disease specific quality of life scores (VEINES-Sym/Qol).

Results: Three hundred and fifteen patients responded, and in 309 a postthrombotic syndrome score was distilled. LET I,II,III $(n=63)$ had an odds ratio of 3.4(1.2-9.2) for predicting severe post-thrombotic syndrome, and LET II $(n=17)$ had an odds ratio of 5.1(1.3-20.8) compared to LET class I $(n=97)$. Both the VEINES-Sym and VEINES-Qol score were significantly lower for LET I,II,III compared to LET I, and LET II compared to LET I.

Conclusion: The LET classification can be used to classify patients according to acute thrombus location and extent. Extensive and centrally located (LET I,II,III) deep venous thrombosis showed the highest probability in developing severe post-thrombotic syndrome and lower disease specific quality of life. 


\section{Introduction}

Post-thrombotic syndrome (PTS) is the most common complication after a deep venous thrombosis (DVT) of the lower extremities.1 PTS is a debilitating chronic disease that occurs in approximately $50 \%$ of patients, when treated with anticoagulation and elastic compression stockings. 2 Additional thrombus removal therapies are used in the acute stage of ilio-femoral DVT to reduce the incidence of PTS.3 These patients are deemed eligible for active lysis of the thrombus. However, also patients with mid-femoral DVT were included in the randomised controlled CaVenT study. Earlier studies have shown higher incidences of PTS with more cranial located thrombi and multilevel DVT.4 Many risk factors have been studied with regard to developing PTS. Residual vein thrombosis after three and six months was a good predictor of PTS.5,6 Identifying patients with a high risk for developing PTS in the acute stage is important to improve the treatment strategy to reduce or prevent PTS. After three weeks, thrombus removal therapies become less successful leaving a short window to decide the treatment strategy. The LET classification was designed to classify patients with DVT in the acute stage in order to identify those patients who can benefit from additional invasive therapies, such as catheter-directed thrombolysis.7 The classification stratifies patients into four groups (class I-IV) in accordance to the location and extent of the thrombosis. Figure 1 shows the LET classification, and Figure 2 shows the distribution of segments per class.

Figure 1. 

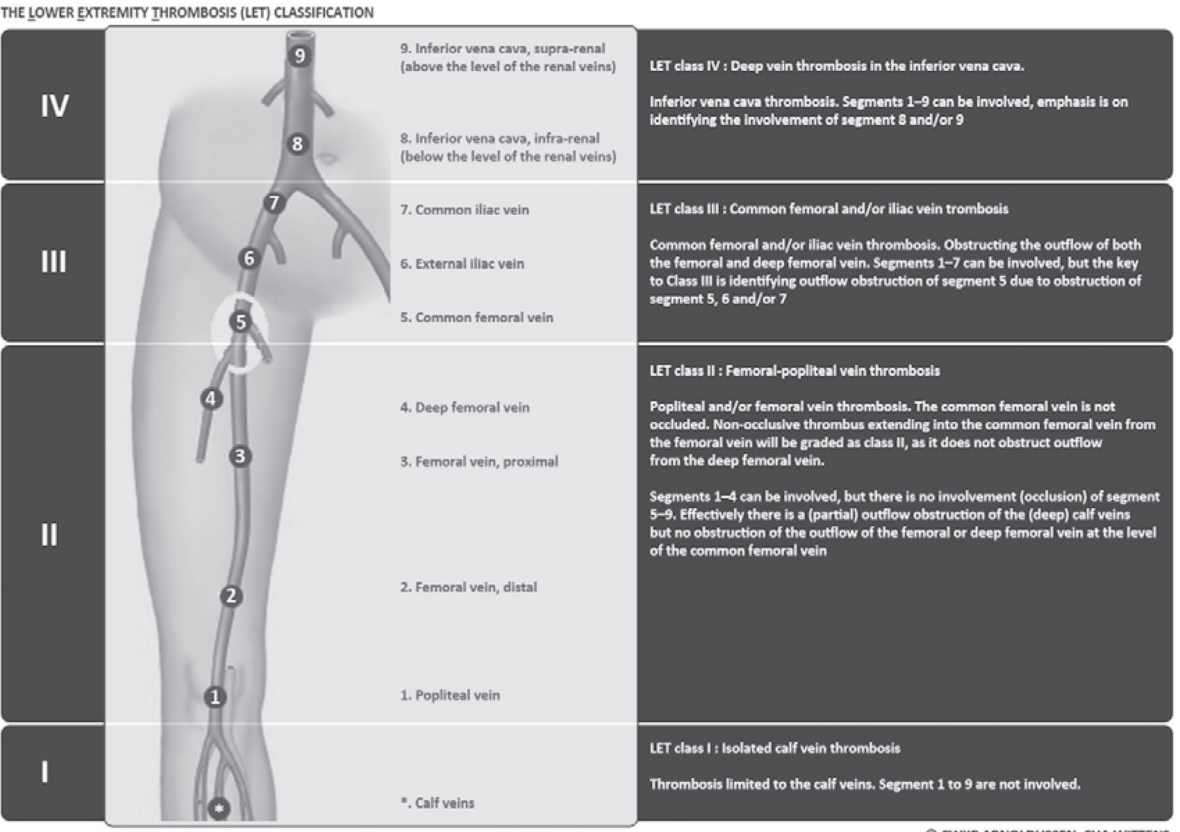

O CWKP ARNOLDUSSEN, CHA WITTENS

The LET classification. 7

Figure 2.

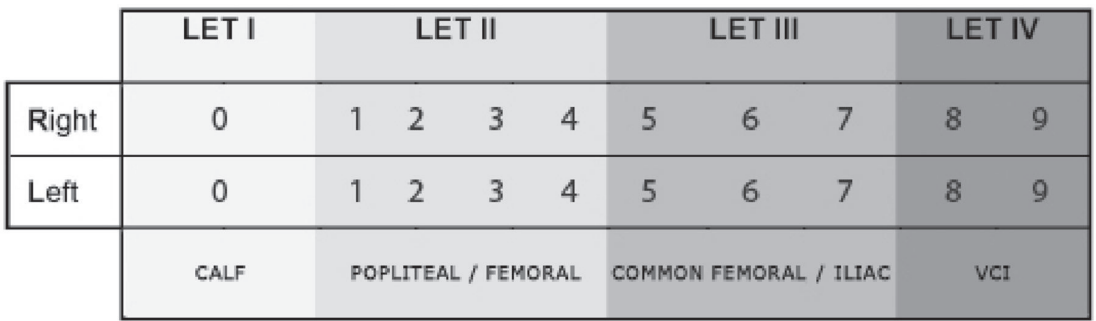

Distribution of segments per LET class. LET III \& IV implies eligibility for CDT based on current evidence (CaVent). LET I \& II can be treated conservatively with therapeutic anticoagulation and compression stockings class II.7

The classification has the potential to provide the physician an easy tool to identify patients in the acute stage at high risk for PTS and might benefit from more aggressive thrombus removal therapies. Patients are characterised by describing all applicable LET classes, for example LET I-II-III in case of involvement of the calf veins, the popliteal vein, the distal femoral vein, the proximal femoral vein and the common femoral, describing not only the proximal extend but also all involved segments. We looked at the ability of the LET classification to predict PTS severity and quality of life. 


\section{Methods}

This study has been realised in close collaboration with the Department of Thoracic and Vascular Surgery at the University Hospital of Antwerp (Belgium) and the Department of Vascular Surgery of the Maastricht University Medical Centre (the Netherlands). Between March 1991 and July 2012, 1338 patients with unilateral acute DVT according to duplex ultrasound investigation and aged $>18$ years had been registered at the University Hospital of Antwerp. Exact localisation and extent of the thrombus according to duplex ultrasound had been represented on a diagram, and all these diagrams had been carefully stored for further analysis. Patients' demographics and data on thrombus location and extent had been registered in a database. Specifics on data acquisition can be found in an earlier reported abstract by Bochanen et al.8

The original description included five segments: calf vein, popliteal vein, femoral vein, common femoral vein and iliac vein. Bilateral DVT and caval involvement were excluded from the analysis. Anatomical data from the charts were transformed into LET scores. LET class IV is therefore not present in the analysis. Five segments were score, calf vein involvement is class I, popliteal and femoral vein involvement is LET class II, common femoral and iliac vein involvement is LET class III DVT. Possible combinations are LET I LET II, LET III, LET I\&II, LET I\&III, LET I\&II\&III, and LET II\&III.

In January 2014, questionnaires were sent to a selected group of the abovedescribed cohort of patients, to obtain information on complaints, severity of PTS and quality of life. Patients younger than 80 years qualified for the study. Patients had to have a basic understanding of the Dutch language, as the questionnaire was only available in Dutch, which caused a significant loss due to Belgium being bilingual. Patients currently living outside Belgium or the Netherlands were also excluded because of logistic reasons. Records were checked to ensure patients who had passed away were not addressed. In total, 660 patients were invited to participate in the study.

The questionnaire was conceived at the Maastricht University Medical Centre and contained the following items: basic demographic questions, details on DVT treatment, recurrent DVT, the validated Dutch translation of the VEINESSym/Qol questionnaire, 9 and a self-assessment version of the Villalta-scale for PTS in Dutch. The latter allowed us to score PTS using a questionnaire only. The patients were asked to complete the whole questionnaire and send it back to our department for analysis. On a separate form, they were asked to indicate any other known diseases they were suffering from. This information was used to test for possible confounders. 
PTS and disease specific quality of life scores were compared to the different LET scores.

\section{Statistical analysis}

Categorical data are presented as frequencies and percentages, and continuous data are presented as mean values \pm standard deviation (SD) or median values with interquartile ranges (IQR), unless otherwise stated. Analysis of differences between means was calculated by ANOVA and post-hoc least significant difference (LSD) test. Odds ratios (OR) were calculated and corrected for confounding in a multivariate logistic regression model. We corrected for age, duration of follow-up, BMI, type 2 diabetes, current use of compression stockings, current use of anticoagulation, orthopedic disease, peripheral arterial disease, recurrent ipsilateral DVT and recurrent contralateral DVT. Analyses were carried out with SPSS version 22 (SPSS version 22.0.0; SPSS, Chicago, IL). P values $\leq .05$ were considered to indicate statistical significance.

\section{Results}

Of the 660 patients who were invited to participate, 315 patients responded with a filled-out questionnaire (47.7\%). Characteristics of the patients are shown in Table 1.

Table 1.

\begin{tabular}{ll}
\hline & $N=3 \mid 5$ Mean \pm SD \\
\hline Gender M/F & $147 / 168$ \\
Age in years at DVT diagnosis & Mean $50.6 \pm 13.3$ \\
BMI & Mean $27.5 \pm 4.6$ \\
Left sided DVT & $56.2 \%(n=177)$ \\
\hline
\end{tabular}

Baseline characteristics of the responders.

In 309 of the 315 received questionnaires, a Villalta score could be calculated. The distribution of PTS severity of all the possible LET classifications is shown in Table 2. None of the patients had a LET I,III DVT.

Table 2. 


\begin{tabular}{|c|c|c|c|}
\hline $\begin{array}{l}\text { LET } \\
\text { classification }\end{array}$ & PTS severity & $\begin{array}{l}\text { Number of } \\
\text { patients }\end{array}$ & $\begin{array}{l}\text { Percentage } \\
\text { within } \\
\text { LET class (\%) }\end{array}$ \\
\hline \multirow{4}{*}{ LET I $(n=97)$} & No PTS & 53 & 54.6 \\
\hline & Mild PTS & 20 & 20.6 \\
\hline & Moderate PTS & 14 & 14.4 \\
\hline & Severe PTS & 10 & 10.3 \\
\hline \multirow[t]{4}{*}{ LET I,II $(n=90)$} & No PTS & 34 & 37.8 \\
\hline & Mild PTS & 24 & 26.7 \\
\hline & Moderate PTS & 18 & 20.0 \\
\hline & Severe PTS & 14 & 15.6 \\
\hline \multirow{4}{*}{$\begin{array}{l}\text { LET I,II,III } \\
\qquad(n=63)\end{array}$} & No PTS & 19 & 30.2 \\
\hline & Mild PTS & 18 & 28.6 \\
\hline & Moderate PTS & 7 & II.I \\
\hline & Severe PTS & 19 & 30.2 \\
\hline \multirow[t]{4}{*}{ LET II $(n=17)$} & No PTS & 4 & 23.5 \\
\hline & Mild PTS & 5 & 29.4 \\
\hline & Moderate PTS & 2 & 11.8 \\
\hline & Severe PTS & 6 & 35.3 \\
\hline \multirow[t]{4}{*}{ LET II,III $(n=26)$} & No PTS & 14 & 53.8 \\
\hline & Mild PTS & 4 & 15.4 \\
\hline & Moderate PTS & 3 & II.5 \\
\hline & Severe PTS & 5 & 19.2 \\
\hline \multirow[t]{4}{*}{ LET III $(n=16)$} & No PTS & 7 & 43.8 \\
\hline & Mild PTS & 4 & 25.0 \\
\hline & Moderate PTS & 2 & 12.5 \\
\hline & Severe PTS & 3 & 18.8 \\
\hline
\end{tabular}

Distribution of PTS severity between the different LET classes.

LET classes and PTS severity

Comparison of the LET classes shows differences in PTS occurrence. Overall PTS occurrence is lowest in LET class I, and highest in LET II and LET I,II,III. Post-hoc LSD tests show a significant difference in overall PTS occurrence between LET I and LET I,II ( $p=0.027)$; LET I vs LET II $(p=0.022)$; LET I vs LET I,II,III ( $p=0.002) ;$ LET II,III vs LET I,II,III $(p=0.03) ;$ LET II,III vs LET II 
( $p=0.047)$. LET I and LET II,III showed lower overall PTS occurrence. Mild and moderate PTS scores did not differ between the LET classes. But regarding the clinically more important severe PTS rates differences were identified. Significantly higher rates of severe PTS were present in LETII vs LET I $(p=0.012)$ and LET I,II ( $p=0.048)$; and also in LET I,II,III vs LET I $(p=0.002)$ and LET I,II $(p=0.026)$. Figure 3 shows the distribution of PTS severity between the LET classes.

Figure 3.

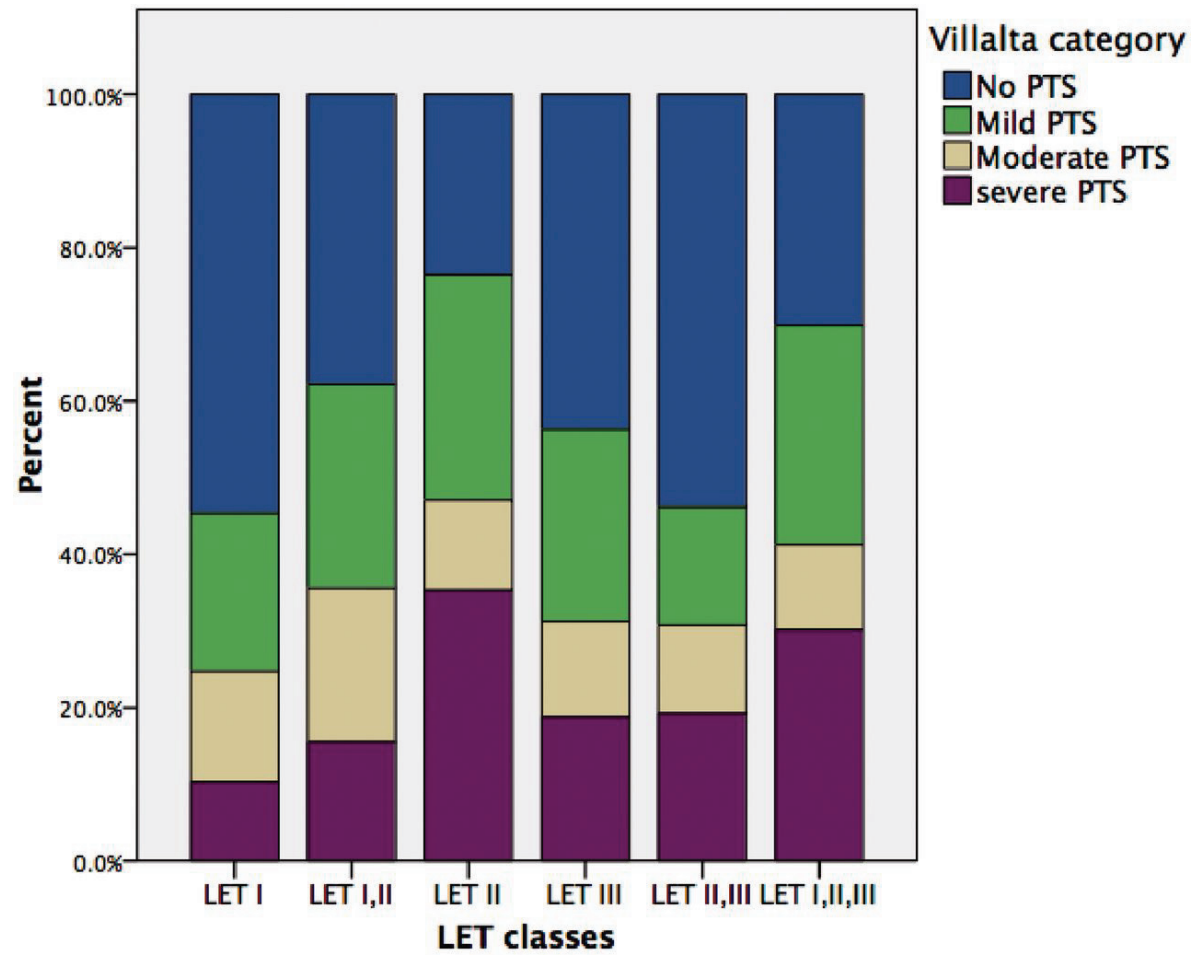

PTS score according to the Villalta scale.

Multivariate logistic regression model for severe PTS

With correction for confounders LET I,II,III had an OR of 3.4(1.2-9.2) for predicting severe PTS, and LET II had an OR of 5.1(1.3-20.8) compared to LET class I. No statistical difference was found in the OR for mild and moderate PTS for any of the LET classes.

\section{Quality of life}

Results for the disease specific quality of life score VEINES-Sym/Qol were similar to the PTS results. Both the VEINES-Sym and VEINES-Qol score were signifi- 
cantly lower for LET I,II,III compared to LET I (sym p $=0.033$, qol p $=0.001$ ) and LET II compared to LET I (sym $p=0.023$, qol $p=0.029$ ). No further significant differences were found regarding QOL.

\section{Discussion}

This is the first report with patient data on the LET classification. The LET classification was first proposed in 2012 and aimed at simplifying the treatment algorithm for the physician in choosing additional therapies for patients with extensive DVT. The LET classification approach was tested to see how the discriminative value was for differences in PTS or QOL. The only differences found in our study were in the total PTS and severe PTS groups. The only differences were located between the LET I and other classes, mainly LET I,II,III and LET II. Similar results were found concerning QOL scores. From previous reports, we expected more severe PTS in the LET I,II,III group, but not from the LET II group.10,11 However, the results from the LET II group could be overestimated due to the low number of patients in this specific subgroup $(n=17)$. The analysis detected differences between the LET classes, where we would have expected the largest difference. The more subtle differences did not become statistically significant. This is probably due to the low number of patients in these subgroups available for analysis. With higher patient numbers, these differences might become significant. Therefore, we state to always use the full description of the classification.

The LET classification allowed for a detailed description of the thrombus location. Furthermore, it provides more discriminative abilities in terms of PTS prediction or quality of life after DVT. This could advocate for the use of the classification in a research setting and possibly also the clinical setting. The LET classification can also be used by describing only the most proximal extension, leaving only a LET I or II or III. For clinical purposes, this simplified version of the LET classification might be very useful to identify patients for more invasive treatments. Analysis of the differences in severe PTS incidence and reduced disease specific QOL scores shows this. The combination of all three subgroups with LET III included resulted in an OR of 3.4(1.2-9.2) for predicting severe PTS compared to LET I and decreased VEINES-Qol scores.

Full description of the LET classification is in our opinion the best score for research purposes and to define which patient is eligible for additional thrombus removal therapy and predict outcome. Catheter-directed thrombolysis is performed in patients with ilio-femoral (LET III) DVT.12 This would correspond to LET I,II,III, LET II,III and LET III patients, which illustrates the potential usefulness of only mentioning the highest LET score to make a clinical deci- 
sion. But in order to predict outcome of such a therapy, which is dependant on the extend of the thrombus the additive information of the complete score is probably very important. In an additional analysis (not shown), there were low numbers for solitary iliac DVT and other subgroups available for analysis. These low numbers indicate that simplified LET reporting could suffice in clinical practice, although this has to be studied more extensively prospectively.

The self reported Villalta scale has been used previously in an cohort of patients after DVT.13 That report states that there is not a big difference to be expected from the use of the self reported score versus the original Villalta scale, as the patients themselves already score one half of the score. Further evaluation of the self reported Villalta scale would be useful, as it is a very convenient tool to assess large groups of patients without a doctor visit.

Strengths of the study include the large number of 315 responders and careful administration of the initial DVT and the use of a multivariate regression model to correct for possible confounding. Furthermore, the combination of PTS score and disease specific QOL is considered the golden standard for diagnosing PTS nowadays. 14

Limits of the study include the use of the self-reported Villalta scale and the low number of patients available for sub-analysis. Smaller differences between all different LET classes could be missed due to low patient numbers.

Further validation of the LET classification should focus on prospective validation. Furthermore, validation of the simplified LET classification, reproducibility and accuracy in clinical practice should be performed. It is a simple tool to implement in daily practice and easy to understand. Separate evaluation of each individual LET class should be performed in relation to PTS occurrence and disease specific quality of life. In future research, use of the original LET classification is encouraged to obtain proper information on thrombus level and extent and to be able to pool data in meta-analyses, which is not possible at the moment due to so many different descriptions of thrombus extent.

\section{Conclusion}

The LET classification can be used to classify patients according to acute thrombus location and extent. Extensive and centrally located (LET I,II,III) DVT showed the highest probability in developing severe PTS and disease specific lower quality of life. 


\section{References:}

1 Kahn SR, Shbaklo H, Lamping DL, et al. Determinants of health-related quality of life during the 2 years following deep vein thrombosis. J Thromb Haemost2008; 6: 1105-1112.

2 Kahn SR, Shapiro S, Wells PS, et al. Compression stockings to prevent post-thrombotic syndrome: a randomised placebo-controlled trial. Lancet2014; 383: 880-888.

3 Enden T, Haig Y, Kløw N-E, et al. Long-term outcome after additional catheter-directed thrombolysis versus standard treatment for acute iliofemoral deep vein thrombosis (the CaVenT study): a randomised controlled trial. Lancet2012; 379: 31-38.

4 Labropoulos N, Waggoner T, Sammis W, et al. The effect of venous thrombus location and extent on the development of post-thrombotic signs and symptoms. J Vasc Surg2008; 48: 407412.

5 Comerota AJ, Grewal N, Martinez JT, et al. Postthrombotic morbidity correlates with residual thrombus following catheter-directed thrombolysis for iliofemoral deep vein thrombosis. J Vasc Surg2012; 53: 768-773.

6 de Wolf MAF, Wittens CHA, Kahn SR. Incidence and risk factors of the post-thrombotic syndrome. Phlebology2012; 27(Suppl): 85-94.

7 Arnoldussen CWKP, Wittens CHA. An imaging approach to deep vein thrombosis and the lower extremity thrombosis classification. Phlebology2012; 27(Suppl): 143-148.

8 Bochanen N, van Schil P and de Maeseneer M. Characterization of thrombus extent in patients with acute lower limb deep vein thrombosis (Abstracts from the 14th Meeting of the European Venous Forum, Belgrade, Serbia, 27-30 June 2013, Paper 1.2). Phlebology 2013; 28: 375-394.

9 van der Velden SK, Biemans AAM, Nijsten T, et al. Translation and validation of the Dutch VEINES-QOL/Sym in varicose vein patients. Phlebology2014; 29: 227-235.

10 Galanaud J-P, Holcroft CA, Rodger MA, et al. Predictors of post-thrombotic syndrome in a population with a first deep vein thrombosis and no primary venous insufficiency. J Thromb Haemost2013; 11: 474-480.

11 Kahn SR, Shrier I, Julian JA, et al. Determinants and time course of the postthrombotic syndrome after acute deep venous thrombosis. Ann Intern Med2008; 149: 698-707.

12 Broholm R,Panduro Jensen L, Baekgaard N. Catheter-directed thrombolysis in the treatment of iliofemoral venous thrombosis. A review. Int Angiol2010; 29: 292-302.

13 Wik HS, Jacobsen AF, Sandvik L, et al. Long-term impact of pregnancy-related venous thrombosis on quality-of-life, general health and functioning: results of a cross-sectional, case control study. BMJ Open 2012; 2: e002048. DOI:10.1136/ bmjopen-2012-002048.

14 Galanaud J-P, Kahn SR. Postthrombotic syndrome: a 2014 update. Curr Opin Cardiol2014; 29: 514-519. 


\section{CHAPTER 5}

Proximal extension of deep venous thrombosis: does it predict postthrombotic syndrome and quality of life at long term?

Short title: initial thrombus extension as predictor of PTS?

Rob H.W. Strijkers

Marianne De Maeseneer

Niels Bochanen

Claudia Baas

Patty J. Nelemans

Carsten W.K.P. Arnoldussen

Arina J. ten Cate-Hoek

Cees H.A. Wittens

Under revision 


\begin{abstract}
Background: Incidence of post-thrombotic syndrome (PTS) after initial deep venous thrombosis (DVT) is high. However simple selection tools to predict severe PTS are lacking. The Lower Extremity Thrombosis (LET) classification for DVT is based on initial thrombus extent. We hypothesized that the LET classification can predict high risk for severe PTS.
\end{abstract}

Methods: Between March 1991 and July 2013 thrombus location and extent of consecutive patients $(n=1338)$ with unilateral DVT had been recorded on a diagram, based on duplex ultrasound findings. From this cohort the records were converted into the LET classification for all patients. In January 2014 a questionnaire was sent to 660 patients, still alive and $<80$ years old. Questions concerned quality of life (Veines-qol score), self-reported Villalta-scale, use of stockings, anticoagulant treatment and comorbidities. Differences in Villalta and VEINES-qol between LET classes were calculated and a multivariate logistic regression analysis was performed.

Results: We received 315 (47.7\%) responses. The initial DVT had been classified as LET I in 100 patients, LET II in 108 and LET III in 107. Median time after DVT was 6 years (3-9). Percentage of patients with severe PTS was 10\%, 18.5\% and $25.2 \%$ for LET I, II, and III, respectively. According to multivariate logistic regression analysis LET II predicted development of severe PTS compared to LET I, with an OR of 1.9 (0.7-4.9), LET III had an OR of 3.4 (1.3-8.8) for predicting severe PTS, compared to LET I. VEINES-qol scores significantly decreased with higher LET $(\mathrm{p}=0.003)$.

Conclusion: Patients with LET III have a threefold higher risk for developing severe PTS compared to LET I. The LET classification provides a useful tool to identify patients at high risk for developing severe PTS and decreased quality of life at long-term follow-up after DVT. 


\section{Introduction}

Deep Venous Thrombosis (DVT) is a common disease in the western world, with an incidence of approximately 1-2 per 1000 per year. ${ }^{1}$ Standard conservative treatment with anticoagulation and compression therapy is effective in preventing thrombus propagation and death from pulmonary embolism (PE). ${ }^{2}$ However a large amount of patients will develop post-thrombotic syndrome (PTS) within 2 years after the initial event. ${ }^{3}$ After cessation of anticoagulation, 1 in 3 patients will experience recurrence of either DVT or PE within 5 years. ${ }^{4}$ The high frequency of long-term sequelae of DVT emphasizes the need for improvement of care in patients with DVT.

PTS frequently develops after DVT in $20-50 \%$ of patients. ${ }^{1,5}$ The exact etiology of PTS is still unknown; however residual vein obstruction and deep venous reflux are essential features in PTS. ${ }^{2,6}$ Several risk factors for developing PTS have been identified. One of the well-known facts is that recurrent ipsilateral DVT increases the risk for developing PTS significantly. 3,7 Another important factor is the level and extent of the initial DVT, which has also been associated with a higher risk for inducing PTS. ${ }^{4,8-10} \mathrm{Kahn}$ et al reported a higher incidence of PTS after an iliofemoral DVT, compared to the risk after calf vein thrombosis. ${ }^{3}$ Lack in uniform reporting of thrombus extent makes pooling and comparing of results of DVT treatment difficult.11 Unfortunately, detailed information on thrombus extension is not always clearly available in literature. To facilitate standardization in DVT reporting, Arnoldussen et al. proposed to use the lower extremity thrombosis (LET) classification. ${ }^{12}$ The LET classification provides a guide for the clinician, which may help to determine if patients could be eligible for additional thrombus removal therapies or not. The CaVent study showed an absolute reduction in the incidence of PTS of $14.4 \%$ in DVT patients treated with additional thrombus removal therapy compared to anticoagulant treatment alone. This study included patients with DVT extending to at least mid-thigh or more proximally. ${ }^{13}$ The LET class is determined by the most cranial extension of the thrombus, as shown in Fig. 1. The LET classification has been designed based on a hemodynamic hypothesis that the severity of DVT, and the risk for developing PTS or recurrent DVT, is related to the most cranial level of thrombus extension: the higher the LET class the higher the incidence and severity of PTS and the lower the associated quality of life due to a more significant impairment of the outflow from the leg. This hypothesis has not yet been validated so far, and therefore the aim of the present study was to correlate the incidence and severity of PTS, and the reduction in quality of life at long-term follow-up after DVT, with the LET class. 
Figure 1

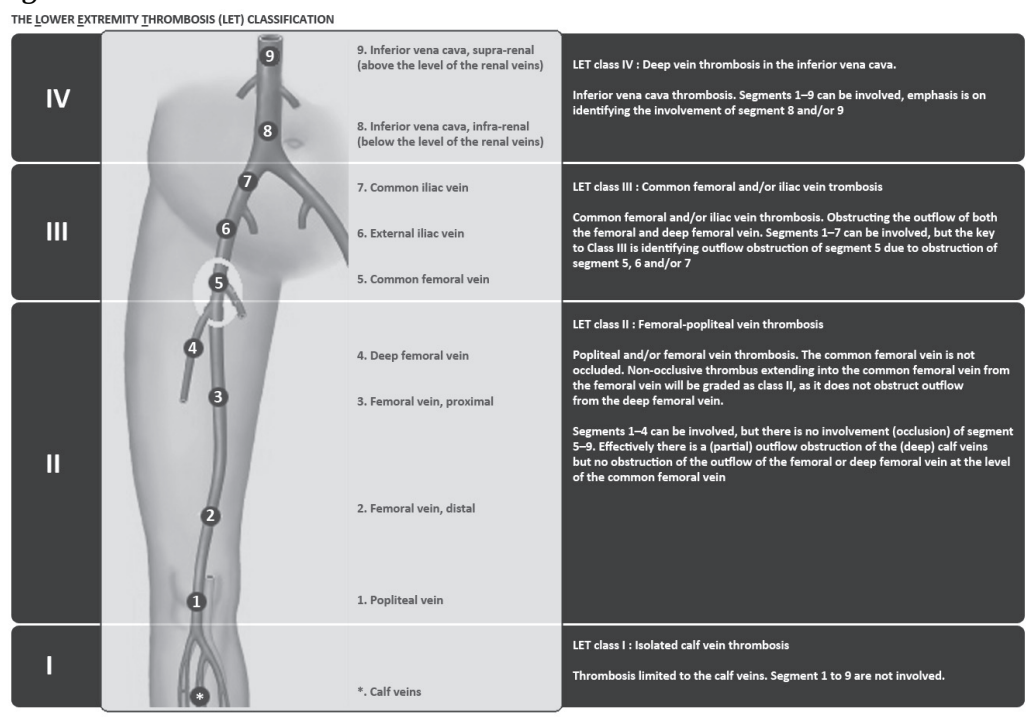

\section{Methods}

This study was performed in close collaboration between the Department of Thoracic and Vascular Surgery at the University Hospital of Antwerp (Belgium) and the Department of Vascular Surgery of the Maastricht University Medical Centre (Netherlands). Between March 1991 and July 2012, 1338 patients with unilateral acute DVT according to duplex ultrasound investigation and aged > 18 years had been registered at the University Hospital of Antwerp. Exact localization and extent of the thrombus according to duplex ultrasound had been represented on a diagram for each patient, and all these diagrams had been carefully stored. Data on thrombus location and extent, based on the available diagrams, as well as patient's demographics, had been registered in a database for further analysis. ${ }^{14}$

For the present study the original subdivision of thrombus location in five segments (calf veins, popliteal vein, femoral vein, common femoral vein [CFV], iliac and/or inferior vena cava [IVC] caval vein) was converted into the corresponding LET class in the following manner: partially or fully obstructed calf veins without any obstruction of the popliteal vein was scored as LET class I. Partial or full obstruction in the popliteal and femoral vein (with or without calf vein obstruction) and without obstruction more cranially was scored as LET class II. Partial and/or full obstruction of CFV, and/or iliac veins (and/or IVC) was scored as LET class III. As information regarding involvement of the deep femoral vein was not available in all files we chose to include any obstruction of the 
CFV into LET class III. Fig.1 This is slightly different from the original LET classification (Fig. 1). ${ }^{15}$ The study protocol was approved by the ethical committee of the University Hospital Antwerp (number:B300201420025).

\section{Questionnaire follow-up}

In January 2014 questionnaires were sent to a selected group of the abovedescribed cohort of patients, to obtain current information on their health status, complaints and severity of PTS. Information about therapy received for the initial DVT and about comorbid diseases was also gathered. Patients qualifying for the questionnaires had to be younger than 80 years on 1-1-2014, and had to have a basic understanding of the Dutch language, as the questionnaire was only available in Dutch. Patients currently living outside Belgium or the Netherlands were also excluded because of logistic reasons. Before sending out the questionnaires records had been checked to ensure patients who had died, were not addressed.

The questionnaire was conceived at the Maastricht University Medical Centre and contained the following items: basic demographic questions, details on DVT treatment, recurrent DVT, the Dutch Short Form -36 quality of life questionnaire version 2 (SF-36v2), the Dutch translation of the VEINES-sym/qol questionnaire, and a modified version of the Villalta-scale for PTS in Dutch. ${ }^{16}$ The complete questionnaire is available in the supplement (in Dutch). The patients were asked to complete the whole questionnaire and send it back to our department for analysis. On a separate form they were asked to indicate any other known disease they were suffering from and which specialist they were currently visiting, if any.

\section{Quality of life (QoL)}

Generic QoL was assessed with the SF-36v2 (Short-Form 36). 17,18 The SF-36 is a generic quality of life instrument consisting of 36 items, divided into eight subdomains of health: physical functioning, social functioning, role limitations (physical problems), role limitations (emotional problems), pain, mental health, vitality and general health perception. Each domain has a theoretical score between 0-100 (higher is more favorable for the patient). The score reflects the health perception for that specific domain.

VEINES-sym/qol is a venous disease specific quality of life questionnaire. It consists of 25 items, 10 items are symptom related, 9 items are used to assess limitations in daily activities, 1 item assesses changes over the past year and the last 5 questions cover the psychological impact of venous disease. Two scores are distilled from these questions, The VEINES-sym (about symptoms) 
and the VEINES-qol (about quality of life) score. Scores can be between 0-100 (higher scores represent better QoL of the patient).

The Villalta scale is currently accepted as the standard clinical score for evaluating PTS. ${ }^{19}$ The original Villalta scale consists of two parts. The first part contains patient reported complaints of pain, itching, heaviness, paresthesia, and cramps in the leg. The second part is a physician assessed clinical score of the leg. The physician assesses the following 6 items: pretibial edema, skin induration, hyperpigmentation, redness, venous ectasia, and pain on calf compression. The physician also assesses the presence or absence of a venous leg ulcer. Each item is scored form 0-3 points, zero being not present, 1 is mild, 2 is moderate and 3 is severe. A theoretical total of 33 points can be scored with the Villalta scale. A score between 0-4 is scored as no PTS; 5-9 as mild PTS, 10-14 as moderate PTS, 15 and higher is considered to be severe PTS. If the patient suffers from a venous leg ulcer the Villalta scale immediately turns into 'severe PTS', regardless of the total score. In order to be able to use the Villalta scale in a questionnaire to be completed by the patient we modified the Villalta scale in a way patients would be able to assess the clinical signs (usually registered by the physician) by themselves, while making use of a guiding scale based on illustrative pictures.

\section{Statistical analysis}

Categorical data are presented as frequencies and percentages, and continuous data are presented as mean values \pm standard deviation (SD) or median values with interquartile ranges (IQR), unless otherwise stated. The association between LET class and PTS severity (no, mild, and moderate PTS versus severe PTS) was expressed as odds ratio with 95\% confidence interval. A multivariate logistic regression model was used to enable adjustment for potential confounders. LET classes were coded as dummy variables with class LET I as reference category. The model corrected for age, duration of follow-up, BMI, type 2 diabetes, current use of compression stockings, current use of anticoagulation, orthopedic disease, peripheral arterial disease, recurrent ipsilateral DVT and recurrent contralateral DVT. The Jonckheere Terpstra test was used to test for trends in quality of life score between the three LET classes. Analyses were carried out with commercially available statistical programs: SPSS version 22 (SPSS version 22.0.0; SPSS, Chicago, IL). P values $\leq .05$ were considered to indicate statistical significance.

\section{Results}

In total 660 patients were invited to participate in the study. Figure 2 shows the flow chart of the study. The baseline patient characteristics are presented 
in Table 1. No difference was found between non-responders and the responders. Mean age of the invited group was 49.7year $\pm 13.9,46.7 \%$ was male, and $56.7 \%$ had left-sided DVT. With 315 responding patients we had a response rate of $47.7 \%$. No statistical significant differences were found in mean age, DVT side, and male/female ratio.

figure 2

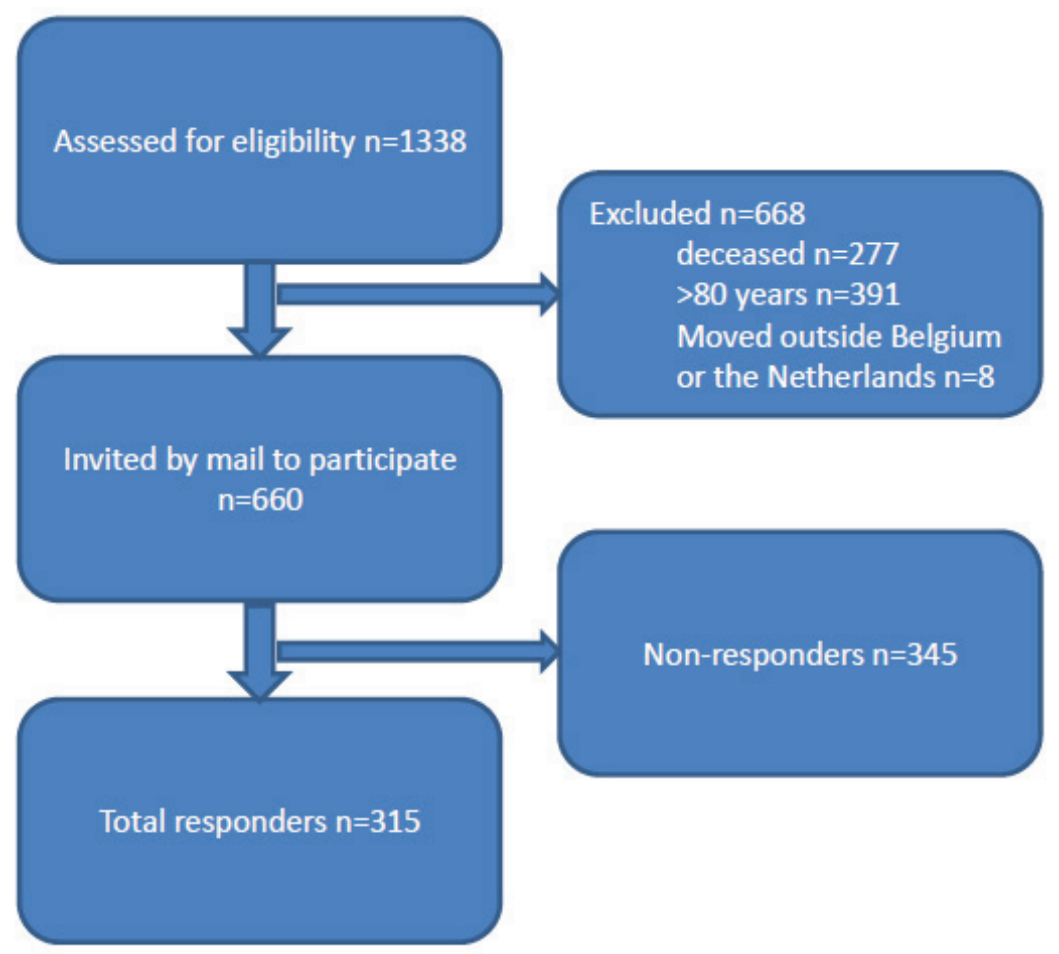


Table 1

\begin{tabular}{|c|c|c|c|}
\hline Baseline patient characteristics & $\begin{array}{l}\text { Responders } \\
\mathrm{N}=315 \\
\text { Mean } \pm \mathrm{SD}\end{array}$ & $\begin{array}{l}\text { Non respond- } \\
\text { ers } \\
\mathrm{N}=345 \\
\text { Mean } \pm \mathrm{SD}\end{array}$ & $\begin{array}{l}\text { p- } \\
\text { value* }\end{array}$ \\
\hline Gender $\mathbf{M} / \mathbf{F}$ & $147 / 168$ & $161 / 184$ & Ns \\
\hline Age in Years at DVT diagnosis & $50.6 \pm 13.3$ & $48.9 \pm 14.1$ & Ns \\
\hline BMI & $27.5 \pm 4.6$ & $\mathrm{~N} / \mathrm{A}$ & \\
\hline Left sided DVT & $56.2 \%(n=177)$ & $57.1 \%(n=197)$ & Ns \\
\hline $\begin{array}{l}\text { Risk factor first DVT } \\
\text { Malignancy } \\
\text { Pregnancy/post partum } \\
\text { Immobilization/surgery } \\
\text { Trauma/fracture } \\
\text { Oral contraceptive use } \\
\text { Other (not specified) } \\
\text { Idiopathic }\end{array}$ & $\begin{array}{l}3.2 \% n=10 \\
5.7 \% n=18 \\
22.2 \% n=70 \\
11.7 \% n=37 \\
4.8 \% n=15 \\
21.9 \% n=69 \\
35.2 \% n=111\end{array}$ & $\mathrm{~N} / \mathrm{A}$ & \\
\hline
\end{tabular}

*Students T test equal variances assumed. Significance is indicated as $\mathrm{P} \leq .05$.

ns $=$ not significant

Results subdivided by LET class

A total of 315 responding patients were classified as LET I, LET II or LET III according to the highest cranial extent of DVT.

Table 2 shows the clinical characteristics of patients according to the LET classes. 
Table 2

\begin{tabular}{|c|c|c|c|c|c|}
\hline $\begin{array}{l}\text { Clinical characteris- } \\
\text { tics }\end{array}$ & $\begin{array}{l}\text { Total } \\
(n=315)\end{array}$ & $\begin{array}{l}\text { LET I } \\
(n=100)\end{array}$ & $\begin{array}{l}\text { LET II } \\
(n=108)\end{array}$ & $\begin{array}{l}\text { LET III } \\
(n=107)\end{array}$ & $\begin{array}{l}\text { p- } \\
\text { val- } \\
\text { ue* }\end{array}$ \\
\hline Age mean \pm SD & $\begin{array}{l}50.1 \pm 13 \\
5\end{array}$ & $\begin{array}{l}51.4 \pm 12 \\
9\end{array}$ & $\begin{array}{l}49.9 \pm 13 . \\
2\end{array}$ & $\begin{array}{l}49.0 \pm 14 \\
4\end{array}$ & ns \\
\hline BMI mean $\pm S D$ & $27.5 \pm 4.6$ & $29.6 \pm 5.1$ & $28.3 \pm 4.0$ & $27.2 \pm 4.7$ & 0.021 \\
\hline $\begin{array}{l}\text { Follow-up length in } \\
\text { months } \\
\text { Median (IQR) }\end{array}$ & $\begin{array}{l}82.5 \\
(46.25- \\
124.5)\end{array}$ & $\begin{array}{l}72(35.5- \\
109)\end{array}$ & $\begin{array}{l}76 \quad(54- \\
124)\end{array}$ & $\begin{array}{l}92 \quad(58- \\
142.5)\end{array}$ & 0.005 \\
\hline $\begin{array}{l}\text { Numeric Villalta } \\
\text { score } \\
\text { Median (IQR) }\end{array}$ & $6(3-11)$ & $4(2-9)$ & $7(3-12)$ & $\begin{array}{l}6.5 \\
12.5)\end{array}$ & 0.002 \\
\hline $\begin{array}{ll}\text { DVT } & \text { recurrence } \\
\text { total } & \\
& \text { Ipsilateral } \\
& \text { Contrala- } \\
& \text { teral } \\
& \text { Bilateral }\end{array}$ & $\begin{array}{l}29.1 \% \\
18.7 \% \\
6 \% \\
4.4 \%\end{array}$ & $\begin{array}{l}32 \% \\
19 \% \\
8 \% \\
5 \%\end{array}$ & $\begin{array}{l}28.7 \% \\
20.4 \% \\
4.6 \% \\
3.7 \%\end{array}$ & $\begin{array}{l}27.1 \% \\
16.8 \% \\
5.6 \% \\
4.7 \%\end{array}$ & $\begin{array}{l}\text { ns } \\
\text { ns } \\
\text { ns } \\
\text { ns }\end{array}$ \\
\hline $\begin{array}{l}\text { Family history of } \\
\text { DVT }\end{array}$ & $27 \%$ & $33 \%$ & $23.1 \%$ & $25.2 \%$ & ns \\
\hline $\begin{array}{l}\text { Family history of } \\
\text { varicosis }\end{array}$ & $57.8 \%$ & $63 \%$ & $53.7 \%$ & $57 \%$ & ns \\
\hline $\begin{array}{l}\text { Still on anticoagula- } \\
\text { tion }\end{array}$ & $51.7 \%$ & $38 \%$ & $54.6 \%$ & $61.7 \%$ & 0.002 \\
\hline $\begin{array}{l}\text { Still using compres- } \\
\text { sion stockings }\end{array}$ & $45.7 \%$ & $35 \%$ & $42.6 \%$ & $58.9 \%$ & 0.002 \\
\hline Type 2 diabetes & $9.2 \%$ & $7 \%$ & $6.5 \%$ & $14 \%$ & ns \\
\hline $\begin{array}{l}\text { Comorbidity re- } \\
\text { ported }\end{array}$ & $56.8 \%$ & $54 \%$ & $50.9 \%$ & $65.4 \%$ & ns \\
\hline $\begin{array}{l}\text { Peripheral arterial } \\
\text { disease }\end{array}$ & $17.1 \%$ & $16 \%$ & $18.5 \%$ & $16.8 \%$ & ns \\
\hline Orthopedic disease & $20.6 \%$ & $24 \%$ & $17.6 \%$ & $20.6 \%$ & ns \\
\hline Venous ulcer & $5.4 \%$ & $6 \%$ & $2.8 \%$ & $7.5 \%$ & ns \\
\hline
\end{tabular}

*Kruskal-Wallis test significance is indicated as $\mathrm{P} \leq .05$

ns= not significant 


\section{Univariate analysis of PTS and LET classes}

In 309 responders a Villalta score could be calculated. In patients with LET I the incidence of PTS was (45.4\%) compared to LET classes II (64.5\%) and III $(61.9 \%)(\mathrm{p}<0.01)$. A univariate logistic regression analysis which included only LET class as independent variable and no PTS versus mild, moderate, and severe PTS as dependent variable resulted in crude odds ratios (not adjusted for potential confounders) of $\mathrm{OR}=2.1$ with a $95 \%$ confidence interval $(95 \% \mathrm{CI}) 1.2$ 3.6 for LET II versus LET I and an OR of 1.9 (95\% CI 1.1-3.3) for LET III vs LET I. With regard to severe PTS vs no, mild and moderate PTS the OR between LET I and LET II was 2.0 (95\% CI 0.9-4.6) and the OR between LET I and LET III was 3.0 (95\% CI 1.4-6.7). Figure 3 shows the distribution of PTS categories according to LET class.

Figure 3

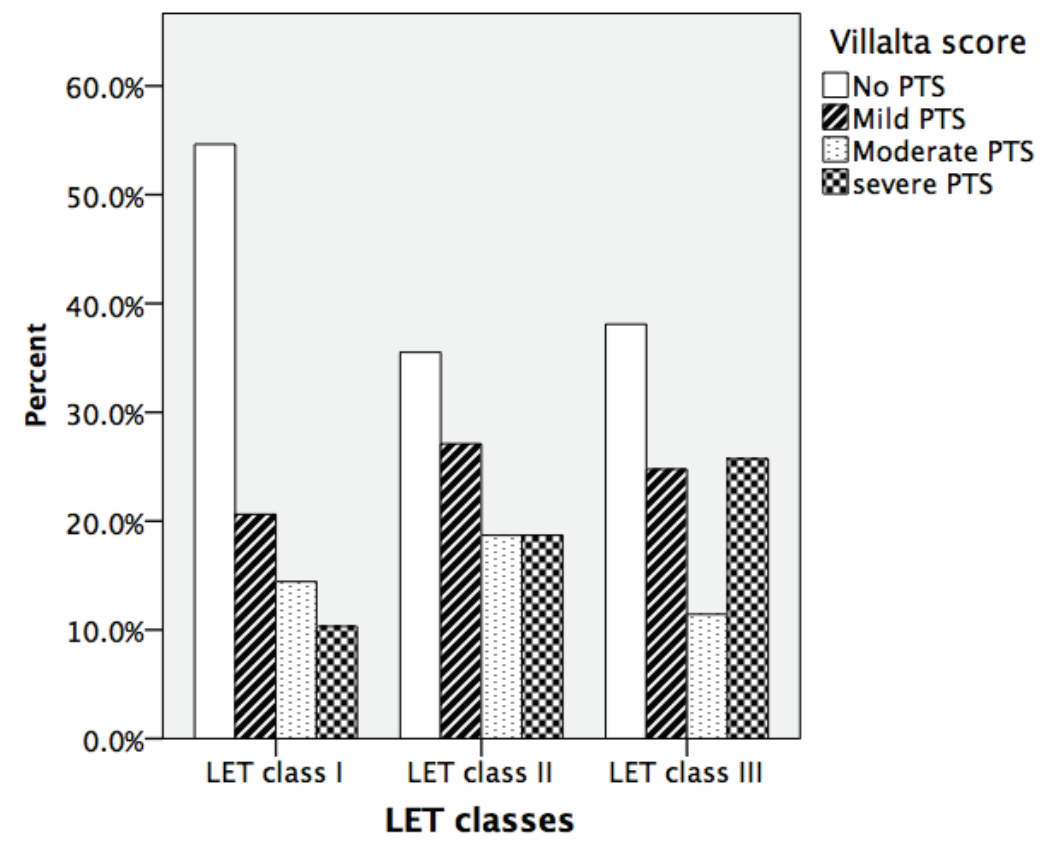

Multivariate regression analysis

Multivariate logistic regression analysis with severe PTS versus no, mild or moderate PTS as dependent variable and adjustment for the predefined potential confounders resulted in an $\mathrm{OR}=1.9$ (95\% CI 0.7-4.9) for LET II compared to LET I and an OR of 3.4 (95\% CI 1.3-8.8) for LET III versus LET I. No statistically significant difference was found between LET classes II and III regarding the risk of severe PTS. 
LET classes and quality of life

Although SF-36 subdomains scores were lower in LET classes II and III compared to LET I, none of these differences showed statistical significance. SF-36 results for all domains are shown in table 3 . For the combined physical component score and mental component score this was also the case. The disease specific VEINES-sym/qol results were fairly similar and showed the same pattern as the SF-36. LET class I had mean scores $( \pm$ SD) of $52( \pm 8.8)$ for VEINESSym and $52.5( \pm 8.9)$ for VEINES-qol, For LET class II this was $48.7( \pm 10.4)$ and $49.3( \pm 10.2)$. LET class III had scores of $49.5( \pm 10.4)$ and $48.5( \pm 10.4)$. Jonckheere's analysis for trend showed a significant trend towards lower VEINES-qol scores with higher LET classes $(\mathrm{p}=0.003)$. Figure 4 shows the LET classification compared to the VEINES-qol score. 
Table 3

\begin{tabular}{|c|c|c|c|c|c|}
\hline $\begin{array}{l}\text { SF-36 individual } \\
\text { components }\end{array}$ & & LET I & LET II & LET III & $\begin{array}{l}\text { P- } \\
\text { value }\end{array}$ \\
\hline $\begin{array}{l}\text { Physical functioning } \\
(n=315)\end{array}$ & $\begin{array}{l}\text { Mean } \\
\pm S D\end{array}$ & $84.5 \pm 56.7$ & $74.2 \pm 58.4$ & $81.7 \pm 69.4$ & Ns \\
\hline $\begin{array}{l}\text { Role limit. (physic. } \\
\text { probl.) }(n=315)\end{array}$ & $\begin{array}{l}\text { Mean } \\
\pm S D\end{array}$ & $76.4 \pm 36.3$ & $76.6 \pm 45.3$ & $67.9 \pm 45.0$ & Ns \\
\hline Pain $(n=305)$ & $\begin{array}{l}\text { Mean } \\
\pm S D\end{array}$ & $75.0 \pm 22.1$ & $69.4 \pm 25.0$ & $71.5 \pm 23.1$ & Ns \\
\hline $\begin{array}{l}\text { General health per- } \\
\text { ception }(n=312)\end{array}$ & $\begin{array}{l}\text { Mean } \\
\pm S D\end{array}$ & $61.5 \pm 26.0$ & $59.5 \pm 29.4$ & $56.8 \pm 28.8$ & Ns \\
\hline Vitality (n=315) & $\begin{array}{l}\text { Mean } \\
\pm S D\end{array}$ & $58.7 \pm 38.2$ & $59.1 \pm 42.9$ & $58.8 \pm 42.8$ & Ns \\
\hline $\begin{array}{l}\text { Social functioning } \\
(n=315)\end{array}$ & $\begin{array}{l}\text { Mean } \\
\pm S D\end{array}$ & $83.0 \pm 33.3$ & $81.2 \pm 39.4$ & $79.6 \pm 39.0$ & Ns \\
\hline $\begin{array}{l}\text { Role limit. (emotion- } \\
\text { al probl.) }(n=315)\end{array}$ & $\begin{array}{l}\text { Mean } \\
\pm S D\end{array}$ & $84.0 \pm 43.3$ & $81.7 \pm 48.1$ & $76.0 \pm 44.3$ & Ns \\
\hline $\begin{array}{l}\text { Mental } \quad \text { health } \\
(n=315)\end{array}$ & $\begin{array}{l}\text { Mean } \\
\pm S D\end{array}$ & $63.9 \pm 29.4$ & $62.4 \pm 35.3$ & $57.4 \pm 30.4$ & Ns \\
\hline $\begin{array}{l}\text { Physical } \\
\text { summary } \\
(n=315)\end{array}$ & $\begin{array}{l}\text { Mean } \\
\pm S D\end{array}$ & $50.7 \pm 13.3$ & $48.3 \pm 14.4$ & $49.7 \pm 17.1$ & Ns \\
\hline $\begin{array}{l}\text { Mental component } \\
\text { summary } \\
(n=315)\end{array}$ & $\begin{array}{l}\text { Mean } \\
\pm S D\end{array}$ & $45.5 \pm 17.6$ & $46.0 \pm 18.6$ & $42.7 \pm 17.0$ & Ns \\
\hline
\end{tabular}

Kruskal-Wallis test significance is indicated as $\mathrm{P} \leq .05$

$\mathrm{Ns}=$ not significant 
Figure 4

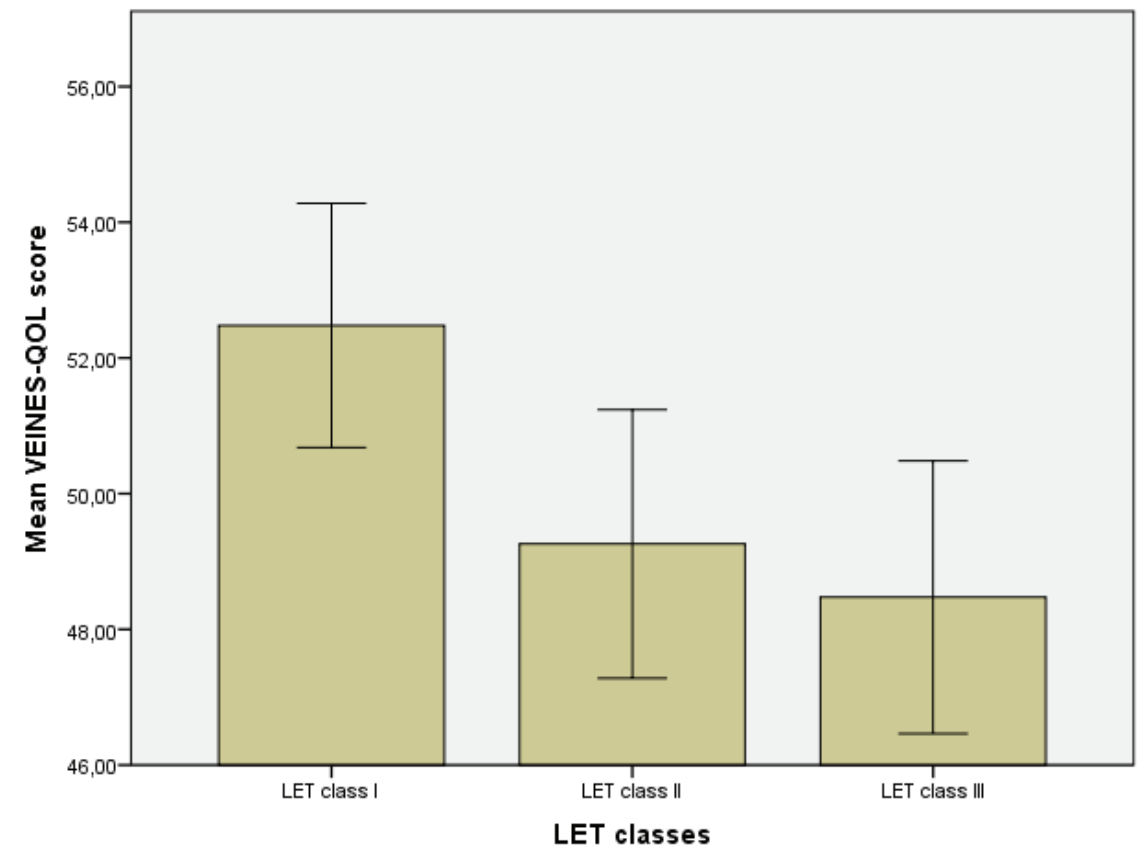

Error Bars: $95 \% \mathrm{Cl}$

\section{Discussion}

We found a two- to threefold higher risk for developing severe PTS for patients with LET II or LET III compared to patients with LET I. The difference between LET class III and II was not significant. This could be explained by limitations in the sample size. Apart from the level of extension of initial DVT, PTS incidence could also be influenced by other factors such as recurrence of DVT and continued use of elastic stockings. In about $30 \%$ of all patients a recurrent event was reported. It may well be that a recurrent DVT extended more cranially than the initial DVT and hence these patients might have acquired a higher LET classification after a subsequent DVT. The incidence and degree of PTS might have corresponded better with the LET classes, if the highest level of extension of recurrent DVT had also been taken into account. Unfortunately for the present analysis, we could not include data on the level of cranial extent of recurrent DVT. In addition, in LET class III more patients continued wearing their elastic stockings regularly. This may have induced some bias, as the treating physician 
may have encouraged patients of LET class III with more persuasion to go on wearing their stocking, in view of the high level of extension of the DVT. The consequent use of stockings may have attenuated postthrombotic symptoms rendering the results regarding PTS severity based on the Villalta scale in LET III less different from those in LET class II than expected.20 However, in the multivariate analysis we corrected for the use of elastic stockings and other potential confounders.

In the present study, we scored PTS by means of an adapted version of the original Villalta scale, a self -reported Villalta scale. By addition of a visual guide to the questions of the clinical scale, we tried to ensure a correct assessment of the clinical situation by the patients themselves. Wik et al. have used the principle of self reported Villalta previously. They reasoned that there should not be a large discrepancy between the original and adapted version of the Villalta scale, because the original version already incorporates a substantial patient assessed part. ${ }^{21}$ It was decided to use this self-reported form of the Villalta scale mainly for efficiency reasons. Further validation of the self-reported Villalta scale should be performed in the future. In order to ascertain the reliability of the results, we combined the Villalta scale with a disease specific quality of life questionnaire as previously suggested. ${ }^{22}$ In our cohort, quality of life scored by VEINES-qol declined with increasing PTS severity according to the self-reported Villalta scale (data not shown). The use of disease specific health questionnaires is recommended in combination with the Villalta-scale, because there is no gold standard for diagnosis of PTS. ${ }^{23,24}$

Thrombus burden and involvement of multiple segments is associated with lower natural resolution of thrombus and higher incidences of PTS.8,2527Therefore, correct identification of the highest extent of DVT is of utmost importance. ${ }^{14}$ Nowadays, thrombus removal techniques are developing rapidly and more patients will be considered eligible for thrombus removal as catheter directed thrombolysis and other pharmacomechanical treatment methods become safer. ${ }^{28}$ Adequate patient selection will be crucial in the future, to decrease the risk of overtreatment or inadequate treatment.

There are several limitations of this study. First of all, it is a retrospective study, only based on limited available data of participating patients. It might certainly be more interesting to validate the LET classification in a prospective study, including the patients at the time of the acute DVT. Follow-up of at least two years after the initial event would be needed to assess the incidence and severity of PTS and associated impairment of quality of life. ${ }^{29}$ Second, the median length of follow-up was long and duration of follow-up varied largely. Although a long follow-up may represent what happens 'in real life' to DVT patients after several years, it is more difficult to draw firm conclusions based on this single 
snapshot at long-term, as comorbidities may have interfered with patients' quality of life. In the multivariate analysis, we tried to correct for these confounders. On the other hand, patients also tend to get used to their limitations, which may have led to underestimation of PTS symptoms and signs, according to the self-reported Villalta scale. Third, with a response rate of almost $48 \%$ we cannot exclude any selection bias in the patients who responded to our invitation. Patients with more severe complaints may have been more motivated to complete the questionnaire we sent them. Also the fact that we had more patients on chronic anticoagulation than in comparable studies may indicate some patient selection. Indeed, patients on chronic anticoagulation need regular medical follow-up, and this may somehow have influenced them to participate in the present study. In addition, further validation of the self-reported Villalta scale should be performed by direct comparison with the original Villalta scale, in which the physician performs the clinical evaluation.

\section{Conclusion}

Patients with LET II or LET III have a two- to threefold higher risk for developing severe PTS at long term compared to LET I. Disease specific quality of life is lower in patients with higher LET classes. The LET classification therefore provides the clinician with an easily applicable tool for identifying patients with a high risk of developing severe post-thrombotic problems.

\section{Acknowledgments:}

We would like to thank the following people for their support in acquiring the data for this article: Irma Wittens, Margriet Stokbroek, Leo Avonds, Sophie Delalieux and Paul van Schil. We also thank the vascular technologists working at the Department of Thoracic and Vascular Surgery of the Antwerp University Hospital between 1991 and 2012, for carefully registering the DVT extent on a diagram for every single patient. 


\section{References:}

1. Nordström M, Lindblad B, Bergqvist D, Kjellström T. A prospective study of the incidence of deep-vein thrombosis within a defined urban population. J Intern Med. 1992 Aug;232(2):15560.

2. Strijkers RHW, Cate-Hoek AJT, Bukkems SFFW, Wittens CHA. Management of deep vein thrombosis and prevention of post-thrombotic syndrome. BMJ. 2011;343:d5916.

3. Kahn SR, Shrier I, Julian JA, Ducruet T, Arsenault L, Miron MJ, et al. Determinants and time course of the postthrombotic syndrome after acute deep venous thrombosis. Ann Intern Med. 2008 Nov 18;149(10):698-707.

4. Prandoni P, Lensing AWA, Cogo A, Cuppini S, Villalta S, Carta M, et al. The long-term clinical course of acute deep venous thrombosis. Ann Intern Med. Am Coll Physicians; 1996;125(1):1.

5. Kahn SR, Shbaklo H, Lamping DL, Holcroft CA, Shrier I, Miron MJ, et al. Determinants of healthrelated quality of life during the 2 years following deep vein thrombosis. Journal of Thrombosis and Haemostasis. 2008 Jul;6(7):1105-12.

6. Meissner MH, Caps MT, Zierler BK, Polissar N, Bergelin RO, Manzo RA, et al. Determinants of chronic venous disease after acute deep venous thrombosis. Journal of Vascular Surgery. 1998 Nov;28(5):826-33.

7. Tick LW, Kramer MHH, Rosendaal FR, Faber WR, Doggen CJM. Risk factors for post-thrombotic syndrome in patients with a first deep venous thrombosis. Journal of Thrombosis and Haemostasis. 2008 Dec;6(12):2075-81.

8. Labropoulos N, Waggoner T, Sammis W, Samali S, Pappas PJ. The effect of venous thrombus location and extent on the development of post-thrombotic signs and symptoms. Journal of Vascular Surgery. 2008 Aug;48(2):407-12.

9. Akesson H, Brudin L, Dahlström JA, Eklof B, Ohlin P, Plate G. Venous function assessed during a 5 year period after acute ilio-femoral venous thrombosis treated with anticoagulation. Eur J Vasc Surg. 1990 Feb;4(1):43-8.

10. Delis KT, Bountouroglou D, Mansfield AO. Venous Claudication in Iliofemoral Thrombosis. Annals of Surgery. 2004 Jan;239(1):118-26.

11. Porter JM, Moneta GL. Reporting standards in venous disease: an update. International Consensus Committee on Chronic Venous Disease. Journal of vascular surgery : official publication, the Society for Vascular Surgery [and] International Society for Cardiovascular Surgery, North American Chapter. 1995. pp. 635-45.

12. Arnoldussen CWKP, Toonder I, Wittens CHA. A novel scoring system for lower-extremity venous pathology analysed using magnetic resonance venography and duplex ultrasound. Phlebology. 2012;27 Suppl 1:163-70.

13. Enden T, Haig Y, Kløw N-E, Slagsvold C-E, Sandvik L, Ghanima W, et al. Long-term outcome after additional catheter-directed thrombolysis versus standard treatment for acute iliofemoral deep vein thrombosis (the CaVenT study): a randomised controlled trial. Lancet. 2012 Jan 7;379(9810):31-8.

14. Bochanen N, van Schil P, De Maeseneer M. Characterization of thrombus extent in patients with acute lower limb deep vein thrombosis [Internet]. Phlebology. 2013. pp. 375-94. Available from: http://phl.sagepub.com/lookup/doi/10.1177/0268355513500449

15. Arnoldussen CWKP, Wittens CHA. An imaging approach to deep vein thrombosis and the lower extremity thrombosis classification. Phlebology. 2012;27 Suppl 1:143-8.

16. van der Velden SK, Biemans AAM, Nijsten T, Sommer A. Translation and validation of the Dutch VEINES-QOL/Sym in varicose vein patients. Phlebology. 2014 May;29(4):227-35.

17. Ware JE, Sherbourne CD. The MOS 36-item short-form health survey (SF-36). I. Conceptual framework and item selection. Med Care. 1992 Jun;30(6):473-83.

18. Lamping DL, Schroter S, Kurz X, Kahn SR, Abenhaim LA. Evaluation of outcomes in chronic venous disorders of the leg: Development of a scientifically rigorous, patient-reported measure of symptoms and quality of life. Journal of Vascular Surgery. 2003 Feb;37(2):410-9. 
19. Strijkers RHW, Wittens CHA, Kahn SR. Villalta scale: goals and limitations. Phlebology. 2012;27 Suppl 1:130-5.

20. Holmes CE, Bambace NM, Lewis P, Callas PW, Cushman M. Efficacy of a short course of complex lymphedema therapy or graduated compression stocking therapy in the treatment of postthrombotic syndrome. Vascular Medicine. 2014 Feb;19(1):42-8.

21. Wik HS, Jacobsen AF, Sandvik L, Sandset PM. Long-term impact of pregnancy-related venous thrombosis on quality-of-life, general health and functioning: results of a cross-sectional, casecontrol study. BMJ Open. 2012;2(6).

22. Villalta S, Bagatella P, Piccioli A, Lensing A, Prins M, Prandoni P. Assessment of validity and reproducibility of a clinical scale for the post-thrombotic syndrome. Haemostasis; 1994;24(158a).

23. Rabinovich A, Kahn SR. How to predict and diagnose the postthrombotic syndrome. Pol Arch Med Wewn. 2014 May 23.

24. Kahn SR, Partsch H, VEDANTHAM S, Prandoni P, Kearon C, Subcommittee on Control of Anticoagulation of the Scientific and Standardization Committee of the International Society on Thrombosis and Haemostasis. Definition of post-thrombotic syndrome of the leg for use in clinical investigations: a recommendation for standardization. Journal of Thrombosis and Haemostasis. 2009 May;7(5):879-83.

25. Sartori M, Favaretto E, Cini M, Legnani C, Palareti G, Cosmi B. D-dimer, FVIII and thrombotic burden in the acute phase of deep vein thrombosis in relation to the risk of post-thrombotic syndrome. Thrombosis Research. 2014 Jun 8;134(2):320-5.

26. Ziegler S, Schillinger M, Maca TH, Minar E. Post-thrombotic syndrome after primary event of deep venous thrombosis 10 to 20 years ago. Thrombosis Research. 2001 Jan 15;101(2):23-33.

27. Johnson BF, Manzo RA, Bergelin RO, Strandness DE. Relationship between changes in the deep venous system and the development of the postthrombotic syndrome after an acute episode of lower limb deep vein thrombosis: a one- to six-year follow-up. Journal of Vascular Surgery. 1995 Feb;21(2):307-12-discussion313.

28. Strijkers RH, Arnoldussen CW, Wittens CH. Thrombectomy without lysis: the future? Phlebology. 2014 May 19;29(1 suppl):125-34.

29. Kahn SR, Comerota AJ, Cushman M, Evans NS, Ginsberg JS, Goldenberg NA, et al. The Postthrombotic Syndrome: Evidence-Based Prevention, Diagnosis, and Treatment Strategies: A Scientific Statement From the American Heart Association. Circulation. 2014 Sep 22. 


\title{
CHAPTER 6
}

\section{Feasibility of identifying deep vein} thrombosis characteristics with contrast enhanced MR-Venography

\author{
Carsten W.K.P. Arnoldussen \\ Rob H.W. Strijkers \\ Dunja M.J. Lambregts \\ Max J. Lahaye \\ Rick de Graaf \\ Cees H.A. Wittens
}

Phlebology 2014, Vol. 29(1S) 119-124 


\begin{abstract}
Purpose: To assess the feasibility of identifying deep vein thrombosis characteristics with contrast enhanced magnetic resonance venography.

Materials and Methods: A total of 53 cases of deep vein thrombosis extending in and/or above the common femoral vein were evaluated by 4 independent observers ( 2 expert, 2 novice) using pre-determined characteristics to determine the thrombosis present to be acute, sub-acute or old. If present, chronic remnants of a previous deep vein thrombosis were reported. Additionally these image qualifications were compared to the reported duration of complaints.

Results: In all cases all observers were able to qualify the thrombosis. The interobserver agreement between the experts was excellent (kappa 0.97) and good between expert and novice (kappa 0.82). Thrombosis identified as acute had an average duration of complaints of 6,5 (2-13) days, sub-acute 13 (8-18) days and old 22 (15-32) days.

Conclusion: Qualification of thrombosis as acute, sub-acute or old and identification of chronic remnants of DVT with CE-MRV using routinely identifiable characteristics is feasible and reproducible with good to excellent interobserver agreement.
\end{abstract}

\title{
Keywords:
}

Venous thrombosis, veins, magnetic resonance imaging, contrast agents 


\section{Introduction}

Deep vein thrombosis affects many patients every year. Despite best medical management with anticoagulation, there is a $30-50 \%$ risk to be subsequently affected by the post-thrombotic syndrome (PTS) resulting in long-term, mostly life-long persistent complaints of leg swelling, pain and potentially venous ulcerations.1,2 For PTS there is no specific treatment for the under-lying venous pathologies and treatment is mostly supportive. Much of the process of thrombus resolution in humans is still unclear but there is supportive evidence that early thrombus resolution reduces the chances of developing PTS, hence techniques such as catheter-directed thrombolysis (CDT) and pharmacomechanical thrombolysis (PMT) have gained more support.3-5 In general, the use of these techniques is limited to patients with acute iliofemoral deep vein thrombosis, defined by complaints 2 weeks and thrombus in the iliac and/or common femoral vein.6-8 This period of 2 weeks is however arbitrary since we have to rely on duration of patient complaints to estimate this period and success with thrombolysis has been seen in patients with complaints for 3-4 weeks. There is currently no objective measurement to define the critical point beyond which thrombolysis is no longer feasible. The transitional process from acute deep vein thrombosis to "old" thrombus and eventually to chronic remnants in the vein and vein wall after DVT,9-12 can take 6 months up to 1 year to stabilize but it has not been established at what point in time after the start of the event the disease is no longer susceptible to thrombolytic therapy. To be able to predict which patients can benefit from catheter directed thrombolysis (CDT) versus those that are unlikely to benefit would be helpful in determining the preferred treatment plan and duration of anticoagulation. Additionally it would be beneficial if patients unsuitable for CDT would be excluded prior to starting the thrombolysis and thus prevent exposure to the serious risks associated with thrombolysis.1 Ideally this measurement would not require any invasive procedure. Imaging with MRI would be a good option if interpretation is consistent and observer independent.

\section{Concept}

MRI as imaging modality for deep vein thrombosis has been investigated for many years.13-16 A number of observations regarding thrombus characteristics identifiable with MRI have so far been described in the literature.17 The first and most commonly acknowledged is that acute thrombus causes vein dilatation due to luminal filling with thrombus material. The second is the visibility of vein wall thickening and edema surrounding the thrombosed veins which has been associated with the inflammatory response to the thrombus.18 The third is a hypothesis that the heterogeneity of the signal in the thrombus 
seen on some MRI sequences is a possible sign of recanalization within the thrombus (channel formation).19 The blood within these channels has a different (higher) signal intensity in comparison to the remaining thrombus. The fourth is the visibility of post-thrombotic remnants in patients after an episode of deep vein thrombosis.20 Different MRI sequences and protocols have been used in the individual studies describing these thrombosis related findings. If we could identify all these findings with one MR protocol, routine evaluation to characterize the thrombosis might be feasible.

If we link these observations with the in situ process in thrombosed veins, we come to the following concept and interpretation of findings (see also Figure 1):

1. Acute DVT is characterized by obstruction and dilatation of the involved vein(s). This can be identified on MRI as a dilated, homogeneously lowintensity vein lumen with a thin enhancing rim (vein wall).

2. In response to the presence of thrombus an inflammatory response takes place with contrast enhanced MRI increased vein wall enhancement and perivenous edema. Simultaneously recanalization takes place, which is visible on MRI as more heterogeneous signal intensity within the thrombus. Since this takes place in response to the acute event of thrombotic occlusion of the vein we define this as 'sub-acute.'

3. Residual thrombus or transformed thrombus into more chronic material can remain in the vein(s) after the thrombus load has decreased and the inflammatory response has subdued with a normalization of the vein lumen size without evidently visible perivenous edema or vein wall thickening. The acute response of inflammation and lysis has seized and a more chronic process of (slow) residual recanalization takes place. For the purpose of this study we defined this as 'old'.

4. Structural remnants (trabeculations/fibrotic strands) develop over time that can be identified as very low-intensity (black) strands on MRI. We define these as 'chronic'.

5. Additionally, these fibrotic stands can be identified as a separate entity next to acute thrombus in patients with recurrent deep vein thrombosis. The very low-intensity strands (chronic component) are visible separately from the (dilated) homogeneously low-intensity vein lumen filling (acute component). We defined this combination of image findings as 'acute-on-chronic'.

Figure 1. Concept of thrombosis characteristics as visualized with contrast enhanced MR-Venography. 


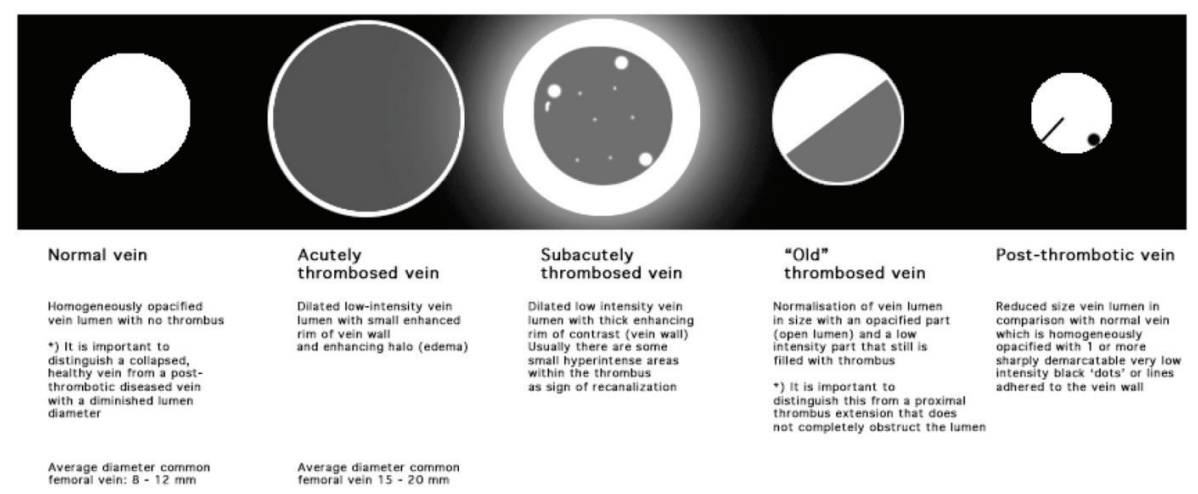

\section{Materials \& Methods}

A retrospective analysis of 53 cases was performed with objectively verified iliofemoral DVT on routine ultra-sound. 34 patients were male, mean age was 47 (range 17-77). All 53 had normal renal function and underwent CE-MRV.

\section{CE-MRV protocol}

All MR examinations were performed on a 1.5-T MRI system (Intera, Philips Medical Systems, Best, The Netherlands). For signal reception a dedicated 12element phased-array peripheral vascular coil with a cranio-caudal coverage of $128 \mathrm{~cm}$ (Philips Medical Systems) was used. Patients were imaged in a supine position. A fixed dose of $10 \mathrm{~mL}$ Gadofosveset Trisodium (Ablavar, Lantheus Medical Imaging, Billerica, MA, USA), a blood pool contrast agent, was administered intravenously as a single dose at a speed of $1.0 \mathrm{~mL} /$ second in the median cubital vein, using a remote controlled injection system (Medrad Spectris, Indianola, PA, USA). Contrast injection was followed by $20 \mathrm{~mL}$ saline flush injected at the same rate. A five-station three-dimensional ultrafast gradient echo (TFE) sequence with fat suppression (SPIR) was used for high-resolution steady-state imaging of the venous vasculature, ensuring coverage of at least the popliteal veins up to the entire IVC. Acquisition parameters were as follows: TR $7.8 \mathrm{~ms}$, TE $3.8 \mathrm{~ms}$, FOV $380 \mathrm{~mm}$, matrix 400, 150 axial slices/station and voxel dimensions (reconstructed) were 0.95 " 0.95 " $1.50 \mathrm{~mm}$ for all stations. Parallel imaging (sensitivity encoding, SENSE) was applied to reduce scan time (SENSE factor 2 in the anterior-posterior direction). For optimal signal intensity and reducing bowel and respiratory artifacts, a NSA of 2 was used. Total acquisition time for five stations was approximately 17 minutes.

\section{Image assessment}

Interpretation of studies was performed in 3 steps. Initially observer 1 (CA) interpreted all studies to evaluate if distinguishing the above-mentioned char- 
acteristics was feasible. The next step was instruction of observer 2 (RdG) by observer 1 with regard to image interpretation. Criteria on how to interpret the images including example images are shown in Figures 2 to 5 . Both observers 1 and 2 had extensive experience with evaluating MR-Venography studies and vascular MR studies in general. The next step was instruction of observer 3 (DL) and 4 (ML) by observer 1 . Observers 3 and 4 were inexperienced with evaluating MR-Venography studies and only had limited experience with vascular MR studies in general. All observers had access to all images in the study, which included axial and coronal reconstructions. Interpretation was done at the level of the common femoral vein. All 4 observers were blinded for the other results and had no access to the patients' clinical history, duplex ultrasound findings or conventional venography studies performed. All observers characterized the common femoral vein on the thrombosed side as either acute, subacute or old thrombosis. Additionally they were asked to identify any signs of acute-on-chronic thrombotic disease separately.

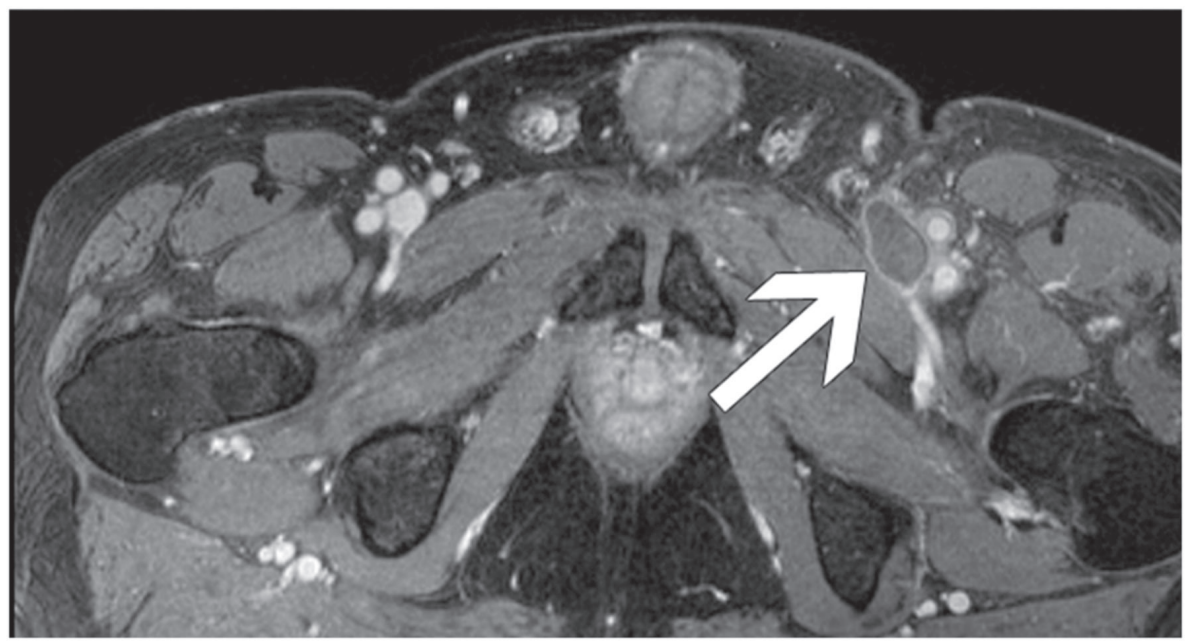

Figure 2. Acute deep vein thrombosis (left common femoral vein): An enlarged, dilated vein with homogenous low (hypo-intense) signal intensity surrounded by a thin enhancing rim of contrast (vein wall) can be seen (arrow). 


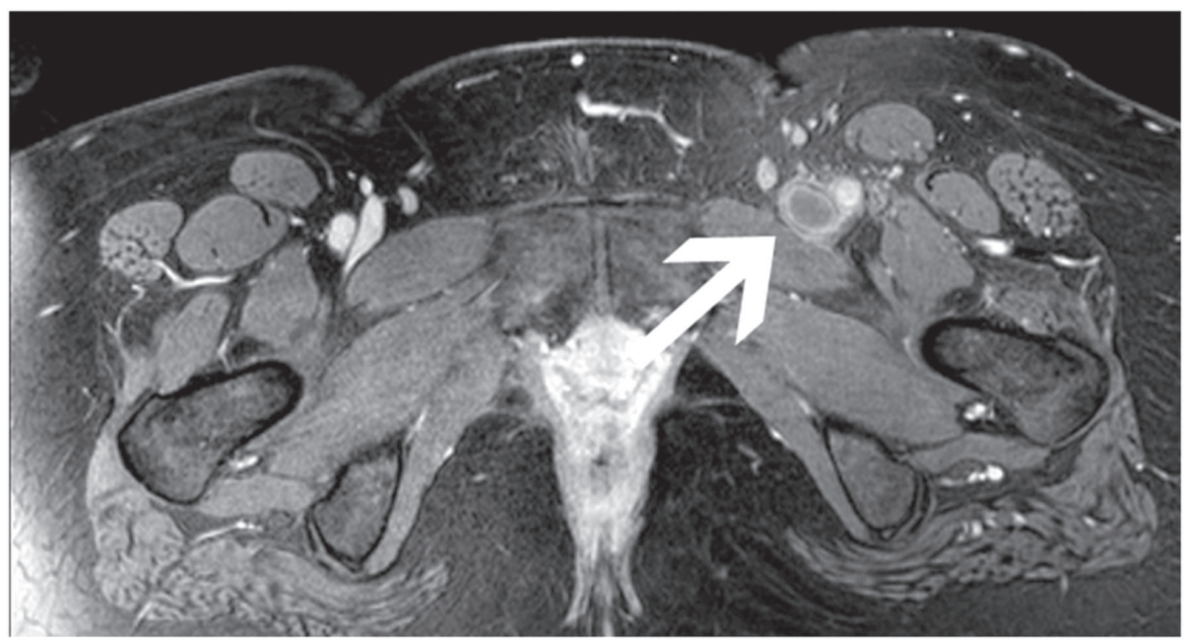

Figure 3. Sub-acute deep vein thrombosis (left common femoral vein): An enlarged dilated vein with a heterogeneous signal intensity surrounded by a thick enhancing rim of contrast (vein wall and surrounding edema) can be seen with some heterogeneity within the thrombus as sign of recanalization (arrow).

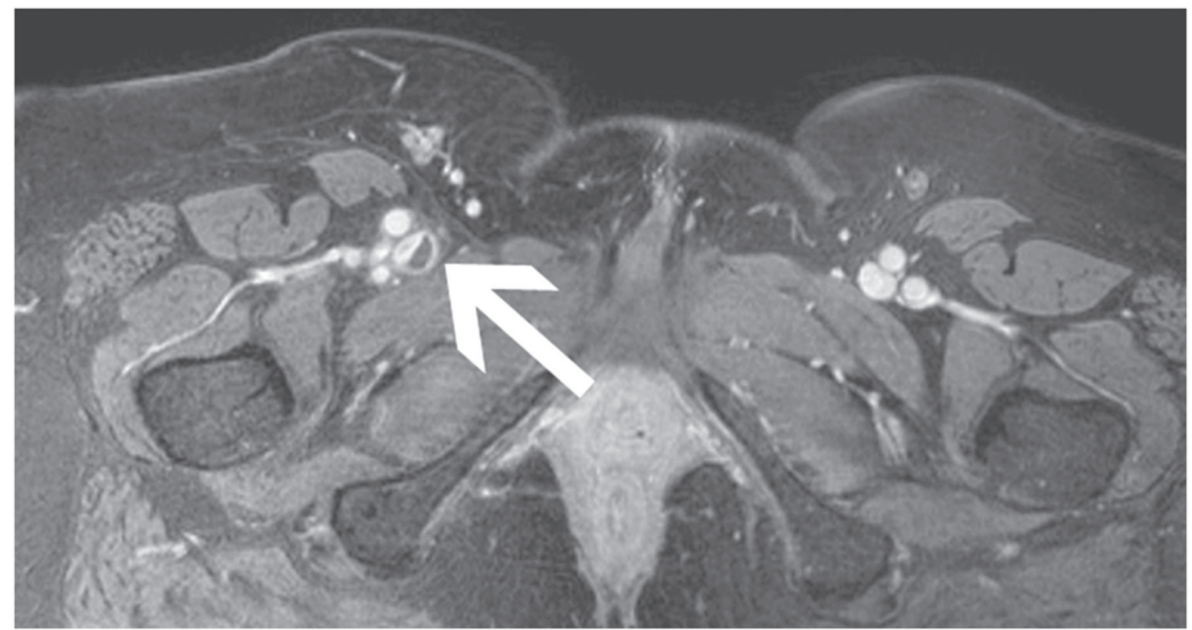

Figure 4. "Old" deep vein thrombosis (right common femoral vein): A normalized caliber vein with heterogeneous and/or hypo-intense material in the vein can be seen. There is no evident enhancing rim of contrast visible, there is no apparent edema (arrow). 


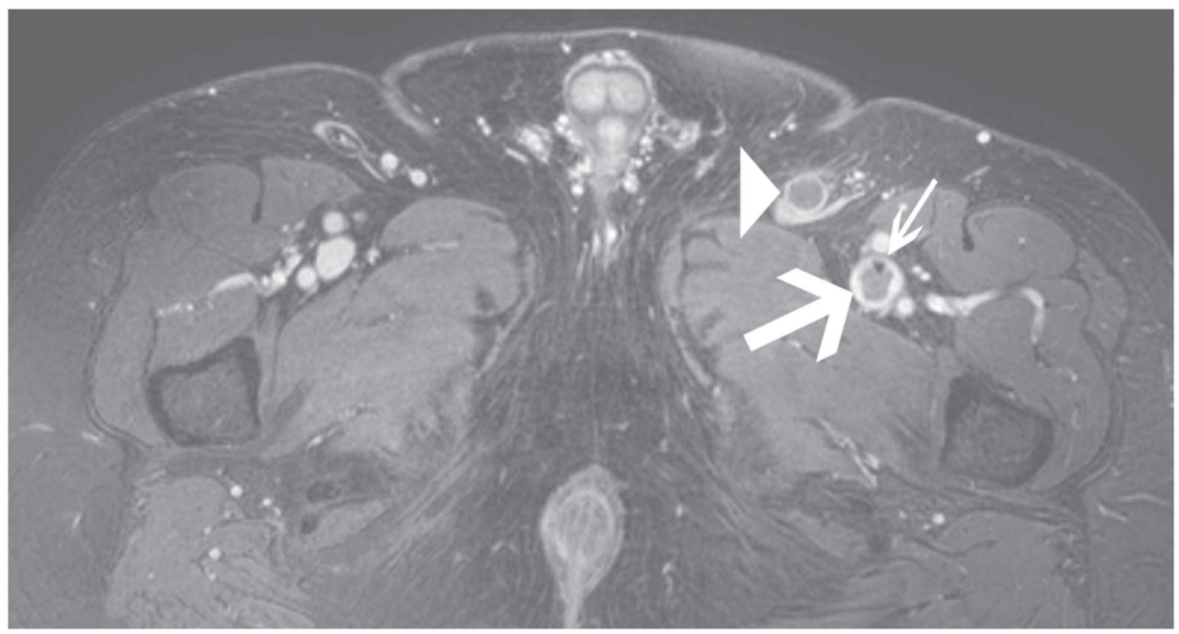

Figure 5. Acute on chronic (left common femoral vein): Adjacent to or in the vein wall, a dark, sharply demarcated dot or strand is visible (old- or postthrombotic material) (thin arrow). The vein lumen is filled with homogenous low (hypo-intense) signal corresponding with the acute component (thick arrow). There is also an acute thrombosis of the great saphenous vein (arrowhead).

Table 1. Findings of all 4 observers.

\begin{tabular}{|c|c|c|c|c|}
\hline & $\begin{array}{l}\text { Observer I } \\
(\mathrm{CA})\end{array}$ & $\begin{array}{l}\text { Observer } 2 \\
\text { (RdG) }\end{array}$ & $\begin{array}{l}\text { Observer } 3 \\
\text { (DL) }\end{array}$ & $\begin{array}{l}\text { Observer } 4 \\
(M L)\end{array}$ \\
\hline Inconclusive & I & I & I & I \\
\hline Acute & 14 & 15 & 19 & 17 \\
\hline Sub-acute & 30 & 29 & 24 & 26 \\
\hline Old & 9 & 9 & 10 & 10 \\
\hline $\begin{array}{l}\text { Acute on } \\
\text { chronic }(*)\end{array}$ & 2 & 2 & 2 & 2 \\
\hline \multicolumn{5}{|c|}{$\begin{array}{l}\text { (*) Patients with acute on chronic disease are listed twice, both as acute } \\
\text { and acute on chronic). } \\
\left({ }^{*}\right) \text { Patients with acute on chronic disease are listed twice, both as acute and } \\
\text { acute on chronic). }\end{array}$} \\
\hline \multicolumn{5}{|c|}{$\begin{array}{l}\text { In addition to the comparison of the results of the } 4 \text { observers, the results of } \\
\text { observer } 1 \text { were compared with the reported duration of complaints in the } \\
\text { patient's clinical history. }\end{array}$} \\
\hline
\end{tabular}




\section{Data analysis}

The statistical analyses were performed using the statistical package SPSS version 18 (SPSS, Chicago, IL, USA). Interobserver agreement was calculated using the kappa statistic. For the purpose of evaluating the interobserver agreement observer 1 was used as reference to which all other observers were compared.

\section{Results}

All common femoral vein segments were visualized completely. All observers found all but one study to be conclusive. The findings for all 4 observers are presented in Table 1. The interobserver agreement between observer 1 and 2 (experts) was excellent with a kappa of 0.97 (Table 2). De interobserver agreement between observer 1 and 3 was good with a kappa of 0.82 and equal to observer 1 versus 4 (Table 3 ). In table 4 the comparison between image interpretation of observer 1 and reported clinical duration of complaints is listed.

\section{Table 2}

\section{Cross tabulation observer $I$ and 2}

\begin{tabular}{lccccc}
\hline & \multicolumn{2}{c}{$\operatorname{RdG}(2)$} & & \\
\cline { 3 - 5 } & & 1.00 & 2.00 & 3.00 & Total \\
\hline CA (I) & 1.00 & 14 & 0 & 0 & 14 \\
& 2.00 & 1 & 29 & 0 & 30 \\
Total & 3.00 & 0 & 0 & 9 & 9 \\
& & 15 & 29 & 9 & 53
\end{tabular}

Symmetric Measures observer I vs 2

\begin{tabular}{lllccc}
\hline Observer I vs Observer 2 & & Value & Asymp. Std. Error $^{\mathrm{a}}$ & Approx. T $^{\mathrm{b}}$ & Approx. Sig. $^{\text {Measure of Agreement }}$ \\
Kappa & .969 & .031 & 10.005 & .000 \\
N of Valid Cases & & 53 & & & \\
\hline
\end{tabular}

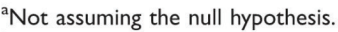

bUsing the asymptotic standard error assuming the null hypothesis.

Table 3. Comparison of findings between observers 1, 3 and 4 .

\section{Symmetric Measures observer I vs 3}

\begin{tabular}{llllll}
\hline Observer I vs Observer 3 & & Value & Asymp. Std. Error $^{\mathrm{a}}$ & Approx. T $^{\mathrm{b}}$ & Approx. Sig. $^{\text {Appra }}$ \\
\hline Measure of Agreement & .824 & .068 & 8.708 & .000 \\
N of Valid Cases & 53 & & &
\end{tabular}

Symmetric Measures observer I vs 4

\begin{tabular}{|c|c|c|c|c|c|}
\hline Observer I vs Observer 4 & & Value & Asymp. Std. Error ${ }^{a}$ & Approx. $\mathrm{T}^{\mathrm{b}}$ & Approx. Sig. \\
\hline Measure of Agreement & Kappa & .821 & .070 & 8.578 & .000 \\
\hline $\mathrm{N}$ of Valid Cases & & 53 & & & \\
\hline
\end{tabular}

Table 4. Characterization of thrombus vs reported duration of complaints. 


\begin{tabular}{lcll}
\hline MRI & $\begin{array}{l}\text { Duration of } \\
\text { complaints } \\
\text { (days) }\end{array}$ & $\begin{array}{l}\text { Average } \\
\text { (days) }\end{array}$ & $\begin{array}{l}\text { Known previous } \\
\text { DVT ipsilateral leg } \\
\text { (number of cases) }\end{array}$ \\
\hline Acute & $2-13$ & 6,5 & 0 \\
Sub-acute & $8-18$ & 13 & 0 \\
Old & $15-32$ & 22 & 0 \\
Acute on chronic & $5-8$ & 6,5 & 3 \\
\hline
\end{tabular}

\section{Discussion}

Identifying thrombus characteristics as described in our study with contrast enhanced MR-Venography (CE-MRV) is feasible and has a good to excellent interobserver agreement. Our data suggests that the degree of experience with vascular MR studies made no significant difference for accurate characterization, which makes routine evaluation by general radiologists feasible. There were however a few cases where the novice observers both experienced (and when asked described) issues with the interpretation. In 1 case the thrombus extension was only just into the common femoral vein from the femoral vein and the deep femoral vein was still patent. Distinguishing such an acute thrombus lip from an "old" thrombus can only be done by interpreting the total thrombus extension and surrounding (potentially patent) veins, which was not defined in the characterization protocol of this study. Most differences in interpretation between the expert and novice observers were between acute and sub-acute (Table 1). This was mainly based on interpretation of the vein wall and surrounding edema as either already subtlety present according to the experts or not yet pre-sent according to the novices. When comparing the acute-on-chronic findings with the clinically reported previous DVT events on the ipsilateral side, 1 DVT was reported that was not seen on MRI in the common femoral vein (which was the evaluated segment in this study). Reevaluating the case we found chronic changes in the femoral vein, but not in the common femoral vein. This particular patient had an acute DVT extending from the deep femoral vein into the common femoral vein and the external iliac vein. The femoral vein was post-thrombotic with a decreased (near pin-point) lumen and no current acute thrombosis.

Unfortunately we did not have the chance to correlate our CE-MRV findings with the actual thrombus present in these patients. Thus at the moment we 
have to accept the potential fact that the recognizable patterns on CE-MRV might not reflect actual critical points in the evolution of thrombosis over time but rather show signs of the local/systemic response to thrombus. Furthermore, assessment in this study was targeted at the common femoral vein in patients with iliofemoral vein thrombosis to ensure reproducibility. Evaluation of larger veins (e.g. iliac or inferior vena cava) is not expected to be problematic, however smaller veins (e.g. distal femoral or popliteal vein) might be less reproducible, in particular in recurrent DVT with vein diameters approaching the scanned voxel size. Identification of chronic venous changes in these smaller veins is not a problem but identifying acute from 'sub-acute' or "old" might prove to be difficult.

Looking at the reported duration of complaints in comparison to the interpretation of the thrombosis characteristics it is interesting to see there is a relationship between duration and characteristics, but also quite an overlap in clinical duration between the different groups defined by MRV. Obviously this raises the question whether interpretation of the MRV studies or reported clinical duration of complaints are inaccurate. When asked in person most patients do not exactly recall when their complaints started unless the com-plaints are very severe or rapid in onset, which makes this particularly unreliable in those patients that have complaints for more than 2 weeks. This supports the fact that in the sub-acute and old groups patients might miss out on the opportunity of receiving CDT because of the duration of complaints or are mistakenly treated with CDT. This finding in particular requires more detailed investigation and correlation with CDT results.

\section{Conclusion}

CE-MR-Venography allows for identification of specific characteristics of the response on and evolution of deep vein thrombosis with good to excellent inter-observer agreement. Correlation with thrombus specimens and consequences of these findings with regard to treatment choices need to be further investigated. 


\section{References:}

1. Hirsh J, Guyatt G, Albers GW, et al. Executive summary: American College of Chest Physicians Evidence-Based Clinical Practice Guidelines (8th edition). Chest 2008; 133: 71S-109S.

2. Lensing AW, Hirsh J and Buller HR. Diagnosis of venous thromboembolism. In: Colma RW, Hirsh J and Marder VJ (eds) Haemostasis And Thrombosis: Basic Principles and Clinical Practice. Philadelphia: JB Lippincott, 1993, pp.1297-321.

3. Henke PK and Comerota AJ. An update on etiology, pre-vention, and therapy of postthrombotic syndrome. J Vasc Surg 2011; 53: 500-9.

4. Enden T1, Haig Y, Kløw NE, et al. Long-term outcome after additional catheter-directed thrombolysis versus standard treatment for acute iliofemoral deep vein throm-bosis (the CaVenT study): a randomised controlled trial. Lancet 2012; 379(9810): 31-8.

5. Vedantham S. Interventional approaches to deep vein thrombosis. Am J Hematol 2012; 87(Suppl 1): S113-8.

6. Haig Y1, Enden T, Slagsvold CE, et al. Determinants of early and long-term efficacy of catheterdirected thromb-olysis in proximal deep vein thrombosis. J Vasc Interv Radiol 2013; 24: 17-24.

7. Kuo WT. Optimizing catheter-directed thrombolysis for acute deep vein thrombosis: validating the open vein hypothesis. J Vasc Interv Radiol 2013; 24: 24-6.

8. Fahrni J, Engelberger RP, Kucher N, Willenberg T and Baumgartner I. Catheter-based treatment of ilio-femoral deep vein thrombosis - an update on current evidence. Vasa 2013; 42: 161-7.

9. Deatrick KB, Elfline M, Baker N, et al. Postthrombotic vein wall remodeling: preliminary observations. J Vasc Surg 2011; 53: 139-46.

10. Roumen-Klappe EM, Janssen MC, Van Rossum J, et al. Inflammation in deep vein thrombosis and the develop-ment of post-thrombotic syndrome: a prospective study. J Thromb Haemost 2009; 7: 582-7.

11. Meissner MH, Manzo RA, Bergelin RO, Markel A and Strandness DE Jr. Deep venous insufficiency: the rela-tionship between lysis and subsequent reflux. J Vasc Surg 1993; 18: 596-605.

12. Kahn SR1, Shrier I, Julian JA, et al. Determinants and time course of the postthrombotic syndrome after acute deep venous thrombosis. Ann Intern Med 2008; 149: 698-707.

13. Spritzer CE, Arata MA and Freed KS. Isolated pelvic deep venous thrombosis: relative frequency as detected with MR imaging. Radiology 2001; 219: 521-5.

14. Spritzer CE. Progress in MR imaging of the venous system. Perspect Vasc Surg Endovasc Ther 2009; 21: 105-16.

15. Aschauer M, Deutschmann HA, Stollberger R, et al. Value of a blood pool contrast agent in MR venography of the lower extremities and pelvis: preliminary results in 12 patients. Magn Reson Med 2003; 50: 993-1002.

16. Enden T1, Stora ${ }^{\circ}$ TH, Nega ${ }^{\circ}$ rd A, et al. Visualization of deep veins and detection of deep vein thrombosis (DVT) with balanced turbo field echo (b-TFE) and contrast-enhanced T1 fast field echo (CE-FFE) using a blood pool agent (BPA). J Magn Reson Imaging 2010; 31: 416-24.

17. Spritzer CE, Trotter $P$ and Sostman HD. Deep venous thrombosis: gradient-recalled-echo MR imaging changes over time-experience in 10 patients. Radiology 1998; 208: 631-9.

18. Parellada AJ, Morrison WB, Reiter SB, et al. Unsuspected lower extremity deep venous thrombosis simulating musculoskeletal pathology. Skeletal Radiol 2006; 35: 659-64.

19. Erdman WA and Parkey RW. MR imaging of deep venous thrombosis. AJR Am J Roentgenol 1990; 155: 897.

20. Arnoldussen CW, de Graaf R, Wittens CH and de Haan MW. Value of magnetic resonance venography and com-puted tomographic venography in lower extremity chronic venous disease. Phlebology 2013; 28(Suppl 1): 169-75. 


\section{CHAPTER 7}

\section{Safety and feasibility of ultrasound accelerated catheter directed thrombolysis in deep vein throm- bosis}

Short title: Catheter-directed thrombolysis in DVT

Jochen Grommes

Rob H.W. Strijkers

Andreas. Greiner

A.H. Mahnken

Cees H.A. Wittens

European Journal of Vascular and Endovascular Surgery 2011 Volume 41, Issue 4, Pages 526-532 


\begin{abstract}
Objective:

Despite optimal standard anticoagulant therapy, currently at least one in four patients with primary iliofemoral deep vein thrombosis (DVT) develops a post thrombotic syndrome (PTS) within one year. Removal of thrombus by thrombolytic agents may prevent PTS. The aim of this study was to assess the shortterm safety and efficacy of ultrasound-accelerated catheter-directed thrombolysis (USaccelerated CDT).
\end{abstract}

\title{
Design:
}

Prospective non-randomized interventional study with US-accelerated CDT for DVT.

\section{Results:}

From October 2008 to January 2010, 12 patients with DVT (7 cavaliliofemoropopliteal, 3 iliofemoropopliteal, 1 femoropopliteal, 1 superior caval vein thrombosis), receiving standard anticoagulant and compression therapy, were treated with additional US-accelerated CDT (13 procedures). Thrombolysis was successful in $85 \%$ (11/13), with complete clot lysis (>90\% restored patency) in $77 \%(10 / 13)$. No pulmonary embolism and one bleeding at the catheterinsertion site were observed. In three patients, underlying lesions were successfully treated with balloon angioplasty and stent insertion. Four patients developed early recurrent thrombosis due to untreated residual venous obstruction.

\section{Conclusion:}

US-accelerated CDT may be a safe and promising frontline therapy in patients with DVT. Residual venous obstruction should be treated by angioplasty and stent insertion to avoid early re-thrombosis.

KEYWORDS: Ultrasound-accelerated catheter-directed thrombolysis, deep vein thrombosis, post thrombotic syndrome, stent placement. 


\section{INTRODUCTION}

Patients with acute deep vein thrombosis (DVT) are treated with anticoagulation, compression therapy and mobilisation. ${ }^{1}$ This standard DVT triple therapy decreases mortality by preventing life-threatening pulmonary embolism (PE) and propagation of thrombosis, but has no direct thrombolytic effect. Recanalisation and the preservation of valve function, therefore, depend on the effectiveness of the patient's own fibrinolytic system. This has resulted in high morbidity due to post-thrombotic syndrome (PTS).

Meissner et al. have demonstrated that valve function is more likely to be retained after early clot lysis. ${ }^{2}$ Singh et al. have revealed that the combination of obstruction and reflux, rather than either one of these, significantly increases the risk of developing PTS. ${ }^{3}$ Rapid clot dissolution by early catheter-directed thrombolysis (CDT) before the onset of valvular damage is suggested to be a way to prevent the development of PTS.

A number of methods of CDT are currently available for the treatment of DVT as outlined in a recent publication from Pianta and Thomson. ${ }^{4}$ Motarjeme ${ }^{5}$ and Parikh et al. ${ }^{6}$ were the first to report ultrasound-accelerated (US-accelerated) CDT in DVT. They report significantly higher complete clot lysis rates with USaccelerated CDT compared with standard $\mathrm{CDT}^{7}$ without raising bleeding or thrombo-embolic risk. In vitro studies have demonstrated that high-frequency, low-power microsonic energy improves lysis of the thrombus considerably by increasing the uptake and penetration of thrombolytic drugs into the thrombus. 8 and 9 US-accelerated CDT is effective in the treatment of peripheral arterial occlusions, ${ }^{5}$ massive PE 10 and acute ischaemic stroke. 11 and 12 Therefore, USaccelerated CDT may also be a safe and promising candidate for immediate treatment of DVT, having the benefits of thrombolysis combined with minimising potential side effects. Few data are available concerning the feasibility, safety and efficacy of US-accelerated CDT. Therefore, the aim of this study is to confirm prospectively the short-term feasibility, safety and efficacy of additional US-accelerated CDT in patients with DVT treated using standard DVT therapy. 


\section{MATERIALS AND METHODS}

\section{Patients}

Between October 2008 and January 2010, 12 patients (seven M: five F; median age 44 years (range 5-79)) with symptomatic, duplex and computed tomography (CT)- or magnetic resonance (MR) angiography confirmed DVT and a life expectancy exceeding 6 months were treated with US-accelerated CDT at the University Medical Centre Maastricht (MUMC+, the Netherlands), the University Hospital RWTH Aachen (Germany) and the VU Medical Centre Amsterdam (VUmc, the Netherlands). In five patients, pulmonary embolism, which was confirmed by CT-angiography, was present before the US-accelerated CDT was commenced. One patient developed recurrent thrombosis 4 days after initial successful thrombolysis and received a second thrombolytic procedure. Therefore, 13 cases of US-accelerated CDT were evaluated. Six patients had a recurrent DVT. Exclusion criteria for US-accelerated CDT were gastrointestinal bleeding or a cerebrovascular haemorrhage in the previous year, severe hypertension ( $>180 / 100 \mathrm{~mm} \mathrm{Hg}$ ), active malignancy, surgery in the previous 6 weeks and/or pregnancy. In one case of upper-extremity thrombosis, the innominate vein was also involved. The 12 remaining cases involved lowerextremity DVT. In those, the proximal end of the thrombosis reached into the vena cava inferior in eight, the iliac vein in three and the femoral vein in one case. The age of the thrombus (defined as the number of days between the onset of symptoms and the intervention) was $0-6$ days (3/13), 7-13 days (5/13), 14-20 days (1/13) or $\geq 21$ days (4/13) (Fig. 1 ).

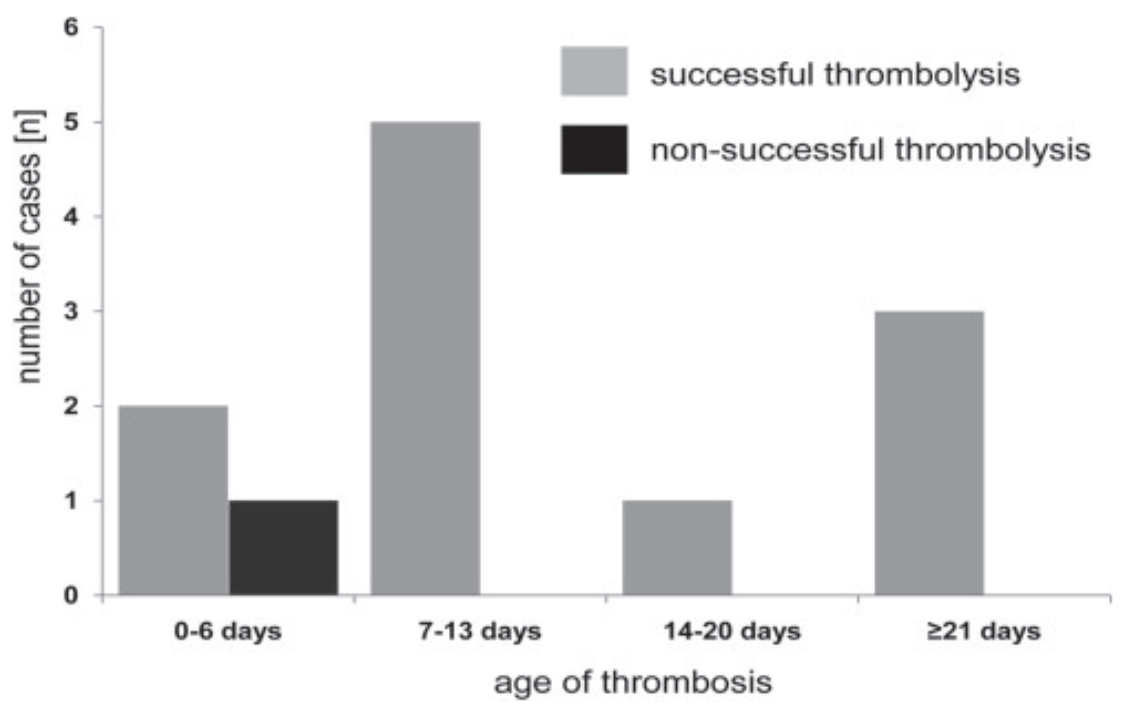

Figure 1. 
Age of thrombus versus success of thrombolysis This diagram shows there was no relation between the age of the thrombus and the success of thrombolysis in this patient series. Successful thrombolysis includes complete and partial clot lysis, defined as $>90 \%$ and $50-90 \%$ restored venous patency respectively.

Eligible patients received US-accelerated CDT with recombinant tissue plasminogen activator (rtPA) (10/13) or urokinase (3/13) combined with standard DVT therapy. Anticoagulation was given according to international guidelines (American College of Chest Physicians, 2008) with the duration of planned treatment being 6 months for idiopathic DVT and 3 months for provoked DVT. ${ }^{1}$ We received approval or gained exemption for the collection of data without patient identification from the institutional review boards of the MUMC+, the University Hospital RWTH Aachen and the VUmc Amsterdam.

\section{US-accelerated CDT}

US-accelerated CDT was performed using the EKOS Endowave ${ }^{\circledR}$ system (EKOS Corporation, Bothell, WA, USA), which combines a targeted-drug-delivery catheter with high-frequency, low-power US energy (Fig. 2). This system uses a standard 0.035-in. guide wire to position the 5.2-F multi-lumen Intelligent Drug Delivery Catheter and matching US coaxial core wires (with available treatment lengths ranging from 6 to $50 \mathrm{~cm}$ ) across the length of the target clot. In all cases, the procedure was performed in an interventional radiology suite. A 7-F sheath and a 0.035-in. hydrophilic guide wire (Terumo Corporation, Shibuyaku, Tokyo, Japan) were placed with the assistance of US-guided popliteal, femoral or jugular venous puncture. The catheter was positioned along the guide wire using X-ray guidance, with the end of the catheter at the proximal end of the thrombus. The guide wire was then pulled out and replaced by the Microsonic core containing a series of US transducer elements ( $2 \mathrm{MHz}, 0.45 \mathrm{~W}$ ) distributed approximately $1.0 \mathrm{~cm}$ apart along its leading tip to deliver evenly US energy radially along the coaxial infusion zone. 

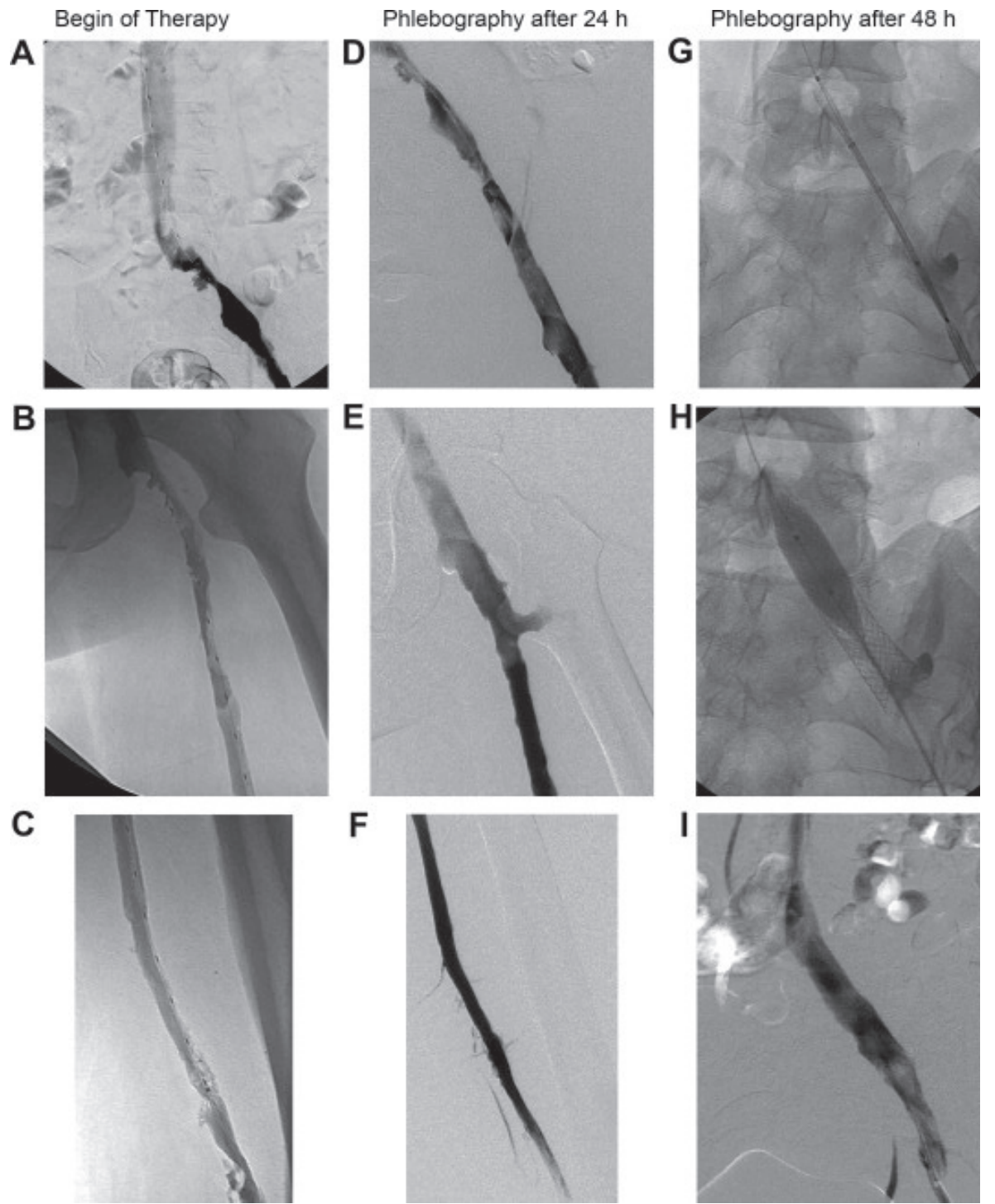

$\mathbf{F}$

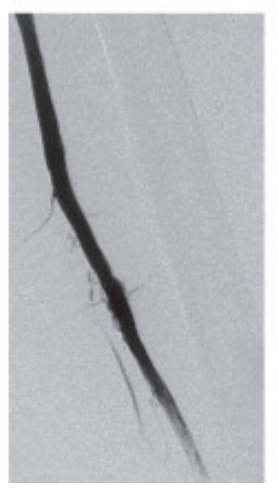

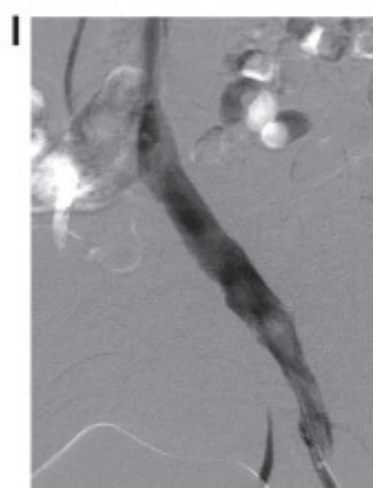

Figure 2.

Pre-, intra-, post-thrombolysis and additional balloon angioplasty with stent insertion phlebograms Phlebographic images from a 36-year old female patient who presented with a post-partum thrombosis from the femoral vein up to the inferior caval vein (A-C). Follow-up phlebogram after $24 \mathrm{~h}$ US-accelerated CDT, showing a May-Thurner Syndrome (D.) and recanalised iliac vein (D) and femoral Vein (E). After balloon angioplasty a Wallstent ${ }^{\circledR}$ (diameter $16 \mathrm{~mm}$, length $60 \mathrm{~mm}$ ) was employed to maintain patency of the treated vein $(\mathrm{G}-\mathrm{I})$. 


\section{Thrombolysis}

After priming the drug lumens of the catheter with heparin (1000 IU), a single bolus of rtPA (5.0 mg) or urokinase (500,000 IU) was administered by slow infusion. Afterwards, continuous infusion of rtPA or urokinase was initiated through the side-hole-delivery infusion catheter at a mean rate of $1.0 \mathrm{mg} \mathrm{h}^{-1}$ rtPA or 100,000 IU urokinase, respectively. Simultaneously, normal saline solution was infused as coolant through the central lumen of the catheter at a rate of $35 \mathrm{ml} \mathrm{h}^{-1}$. Thus, US energy was delivered through the core wire with simultaneous infusion of the thrombolytic drug. All patients were treated with an additional continuous intravenous infusion of heparin through the introducer sheath, which was monitored by assessment of the activated partial thromboplastin time (aPPT). Heparin dosage was adjusted to obtain an aPTT ratio of 1.5-2.5. Follow-up phlebograms were performed on all patients the next day and at 24-h intervals thereafter.

Thrombolysis was terminated, if complete clot lysis was achieved or the maximum infusion period of $72 \mathrm{~h}$ was reached. Hourly and total infused rtPA and urokinase doses and infusion times were recorded; additional angioplasty with or without stent insertion was performed to treat underlying lesions after thrombus lysis had been achieved. Patients received their thrombolytic care at an intermediate care unit and remained hospitalised for one night after termination of thrombolytic treatment, if no complication arose. After discharge, patients were followed up according to the international guidelines for DVT therapy (ACCP 2008). ${ }^{1}$

\section{Definitions}

\section{Thrombolysis success}

Complete clot lysis was defined as $>90 \%$ lysis (restored patency), and partial clot lysis as $50-90 \%$ lysis (restored patency) of the initial thrombus, as assessed on the final phlebograms before additional procedures.

\section{Bleeding}

Bleeding was classified as major, if it was overt with a fall in haemoglobin of $\geq 2 \mathrm{gd}^{-1}$, or when haemorrhage led to transfusion of $\geq 2$ units of packed red blood cells (RBCs) or whole blood. Bleeding situated in a critical organ (intracranial, retroperitoneal or pericardial) or, if it contributed to death, was also defined as a major bleeding. Bleeding was classified as minor, if it was situated near the catheter-insertion site. 


\section{Follow-up}

Every 3 months after discharge, all patients returned to the outpatient department for a follow-up visit, including clinical investigation and duplex ultrasound examination to assess the patency of treated vein segments and the extent of post-thrombotic damage to the deep veins of the lower limb.

\section{RESULTS}

\section{Successful thrombolysis}

Percutaneous catheterisation was successful in 12 procedures, and, in one case, catheterisation of the popliteal vein was achieved by open access.Eleven out of 13 procedures (85\%) resulted in complete clot lysis ( $>90 \%$ restored patency). In one case, only partial clot lysis (50-90\% restored patency) was achieved. In one case, thrombolysis was not successful.The unsuccessful case involved a 5year-old boy. He developed DVT and PE as the consequence of a previously undetected thrombophilia (Factor V Leiden) and immobilisation with a bilateral long-leg spica cast after orthopaedic surgery. Fig. 1 shows the age of the thrombus and the success of thrombolysis. In this small study, no relationship was observed between successful thrombolysis and thrombus age.

\section{Immediate adjunctive procedures}

In three cases, underlying iliac vein stenosis was diagnosed and successfully treated with balloon angioplasty and stent insertion $\left(60 / 16 \mathrm{~mm}\right.$ Wallstent ${ }^{\circledR}$, $40 / 10$ mm Nitinolstent ${ }^{\circledR}$ and $100 / 14$ mm Wallstent ${ }^{\circledR}$, respectively) immediately after thrombolysis. Fig. 2 shows the pre-, intra- and post-thrombolysis phlebograms and the angioplasty with stent insertion of one case involving a 36-year-old female patient with iliocaval vein thrombosis.

Early recurrent thrombosis occurred in four cases of which three were due to inadequate or no treatment of a May-Thurner syndrome (common iliac vein stenosis) demonstrated after completion of thrombolysis.

The first case involved a 17-year-old female patient, who developed a haematoma at the catheter-insertion site, achieved by open access, and in the calf muscle, during thrombolysis, necessitating a 50\% reduction in the hourly thrombolytic drug dose. Although the dose was reduced, successful thrombolysis was achieved. Recurrent thrombosis occurred within a day due to a diagnosed but not immediately treated May-Thurner syndrome. The thrombolytic therapy was not recommenced because of the bleeding complication.

In the second case, which involved a 51-year-old male patient, recurrent thrombosis developed 4 days after initial, successful US-accelerated thrombo- 
lysis in whom the iliac stenosis was not treated immediately. Thrombolysis was repeated with success, followed by angioplasty and stenting of the underlying May-Thurner syndrome and the construction of an arteriovenousfistula in the common femoral vein.

In the third case, a 43-year-old male patient, recurrent thrombosis occurred 1 day after successful thrombolysis, in whom an underlying left-sided MayThurner syndrome was identified and inadequately treated with angioplasty alone without stent placement.

In the fourth case, which involved a 52-year-old female patient, recurrent thrombosis occurred 14 days after successful US-accelerated CDT. This patient had a heparin-induced thrombocytopenia (HIT type II) caused by enoxaparin (Clexane).

\section{Short-term follow-up}

The mean follow-up period was 7 months (range 3-17). In this period, no further occlusion of the venous system occurred in the patients, who were discharged with a patent venous system. In the 5-year-old boy with unsuccessful thrombolysis, the 3- and 6-month US follow-up revealed (spontaneous) recanalisation of the inferior vena cava up to the origin of the left renal vein. The iliac vein remained occluded. In this patient, hypoplasia of the inferior vena cava or a previous DVT probably caused failure of treatment.

In the 17-year-old girl with the untreated reocclusion due to a bleeding complication and a May-Thurner syndrome, 50\% re-canalisation of femoral and iliac vein was found after 6 months. Because she suffered from severe venous claudication, the partially recanalised iliac vein was successfully dilated and stented. Fig. 3 shows the duplex images at 3 months of the case presented in Fig. 2. 

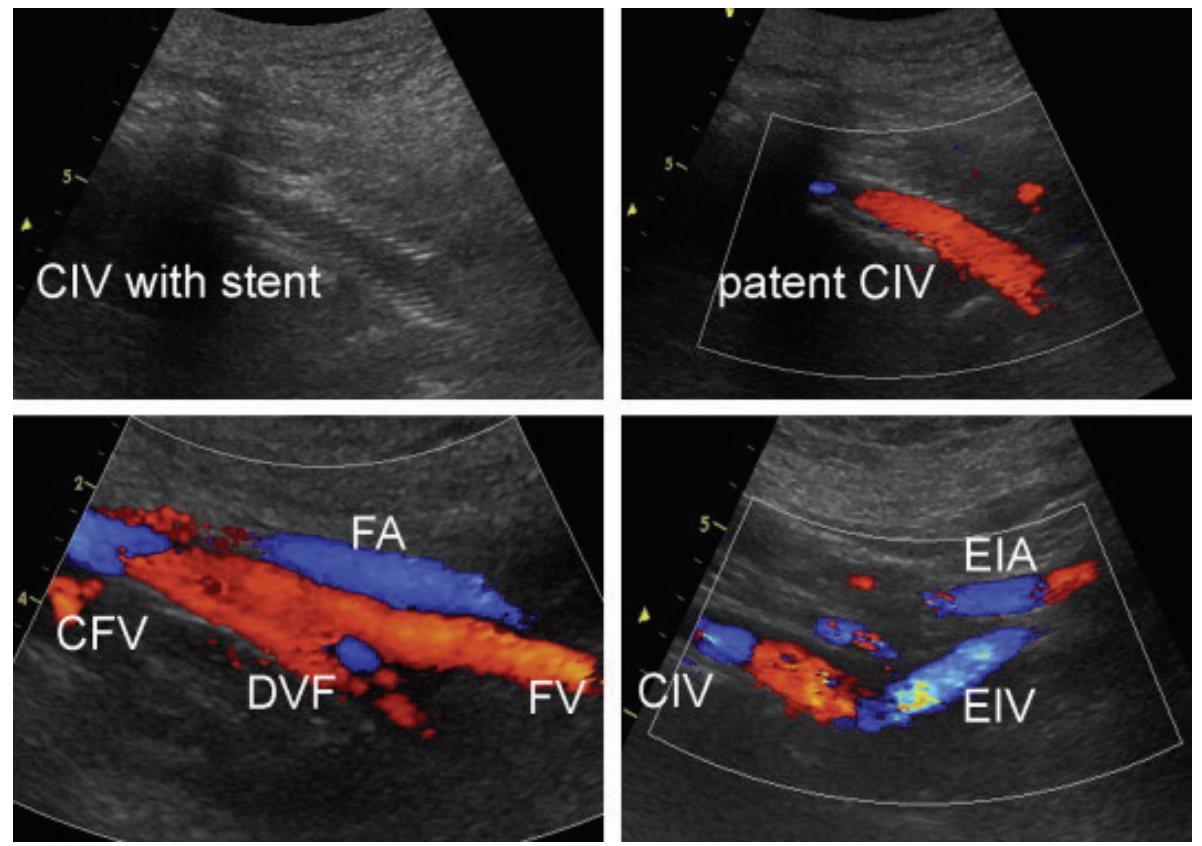

Figure 3.

Duplex images at 3-month follow-up of case presented in Fig. 2 The iliac vein with stent and femoral vein remained patent at 3-month follow-up. (CIV = common iliac vein, EIV = external iliac vein, $\mathrm{CFV}=$ common femoral vein, $\mathrm{FV}=$ femoral vein, $\mathrm{FA}=$ femoral artery, DFV = deep femoral vein).

The patient, who developed a reocclusion due to HIT type 2, displayed 50\% recanalisation of the common iliac and femoral veins at 6 months' follow-up. The iliac veins of the patient with the early reocclusion after thrombolysis and angioplasty without stent placement remained occluded during follow-up.

\section{Complications}

One $(1 / 13 ; 8 \%)$ bleeding complication occurred at the site of the catheterinsertion and in the calf muscle. NoPE was diagnosed during or after the treatment. However, early re-thrombosis was observed in four cases: one due to a HIT type II and three due to an inadequate treatment of residual venous obstruction.

\section{DISCUSSION}

Standard DVT treatment focusses on adequate anticoagulation to prevent PE and thrombus propagation. However, anticoagulation alone has no direct thrombolytic effect. As a result, current DVT treatment often does not restore 
venous patency, and venous valves are permanently damaged. In addition, underlying venous stenoses such as May-Thurner syndrome, which predispose to recurrent thrombosis, are left untreated. The combination of venous obstruction and reflux significantly increases the risk of developing PTS. ${ }^{3}$ Therapy, which can remove the thrombus and restore venous patency, may prevent recurrent thrombosis and PTS.

Our study confirms the promising results of CDT for the treatment of DVT. Most evidence regarding CDT for the treatment of DVT is derived from patient series without controls 5,6 and 13 or cohort studies, ${ }^{7}$ and 14 and little evidence is available from randomised clinical trials. ${ }^{15}$ and 16 Our study obtained a $92 \%$ success rate and highlights the feasibility and capability of the US-accelerated CDT. We observed no PE in our patients during thrombolysis. Bleeding at the catheterinsertion site occurred in one case in whom catheterisation of the popliteal vein was achieved by open access. Despite this one case of bleeding, in whom we did not repeat thrombolysis, no bleeding occurred during US-accelerated CDT.

However, four patients developed early recurrent thrombosis after initial successful thrombolysis. In three patients, underlying stenoses were identified which we considered responsible for the recurrent thrombosis. In the case in which the common iliac vein stenosis was only treated by angioplasty resulting in early reocclusion, a stent should have been inserted immediately to optimise adequate flow in the iliac vein. The 17-year-old girl with common iliac vein stenosis should have been treated immediately by dilatation and stenting after successful thrombolysis. In the third case, the same mistake was made, but, luckily, this was corrected by repeat thrombolysis, stenting and insertion of an arteriovenous fistula.

These three cases highlight the need for immediate treatment of all underlying obstructive lesions. After successful re-canalisation of the venous system, residual venous obstruction should be treated immediately by means of angioplasty and stent insertion to avoid these early reocclusions. Therefore, the centres treating those patients should have the necessary stents immediately at hand to perform these stent placements the moment the underlying cause has been detected, even when thrombolysis has not been completed.

Similar findings have been reported by previous authors such as the series reported in the National Venous Registry by Mewissen et al. ${ }^{7}$ This registry data demonstrated that standard CDT with urokinase and additional stent placement leads to complete (100\%) clot lysis in 31\% and partial (50-99\%) clot lysis in $52 \%$ of cases. The primary patency rate for all patients in this registry was $65 \%$ and $60 \%$ at 6 and 12 months, respectively. These patency rates are similar to the 6-month patency rates reported for standard CDT combined with 
anticoagulation, in two randomised controlled trials by Elsharawy et al. ${ }^{15}$ and Enden et al. ${ }^{16}$ The degree of lysis was found to be a significant predictor of early and continued patency. In cases of complete clot lysis, $75 \%$ of veins remained patent after 1 year, compared with only $32 \%$ of veins in cases of insignificant $(<50 \%)$ lysis. Moreover, subgroup analysis revealed two important observations for iliofemoral DVT: in the subgroup of patients with acute primary iliofemoral DVT, 65\% complete clot lysis was noted, and 1-year patency in this complete lysis group was $96 \%$. This suggests that patients with iliofemoral DVT would benefit most from CDT. In addition, Comerota et al.17 and 18 have demonstrated that successful CDT in iliofemoral DVT significantly improves quality of life (QoL) compared with failed thrombolysis or anticoagulant therapy alone.

An additional advantage of CDT is the ability to detect and treat underlying lesions (e.g., May-Thurner syndrome) immediately after or during thrombolysis. Balloon dilatation with or without stent placement improves long-term patency, ${ }^{7}$ and 19 and can help prevent DVT or prolong the interval to a recurrent DVT. ${ }^{4}$ Mewissen et al. have demonstrated that adjunctive stent placement in the iliac vein significantly improves patency: at 1 year, $74 \%$ of limbs treated with stent placement after thrombolysis remained patent, compared with only $53 \%$ of limbs without stent placement $(\mathrm{P}<0.001) .{ }^{7}$ Bækgaard et al. more recently confirmed, in a large series of patients $(n=101)$, that additional stenting after CDT in iliofemoral DVT results in excellent long-term patency rates $(82 \%$ patent veins at 6 years). 19

Two previous series using US-accelerated CDT have demonstrated considerably increased complete clot lysis rates and fewer complications than standard CDT. 5 and 6 Whereas only $31 \%{ }^{7}$ of patients in the National Venous Registry treated with standard CDT exhibited complete clot lysis, $83 \%^{5}$ versus $85.7 \%{ }^{6}$ of patients treated with US-accelerated CDT using urokinase had complete clot lysis. The major bleeding rate was $11 \%$ and the thrombo-embolic rate $1 \%{ }^{7}$ with standard CDT, compared with $0 \%{ }^{5}$ to $3.8 \%$ and $6 \%$, respectively, in USaccelerated CDT. 5 and 6

\section{Conclusion}

US-accelerated CDT using EKOS Endowave ${ }^{\circledR}$ was found to be a feasible technique for managing iliofemoral venous thrombosis resulting in low morbidity and mortality. All underlying obstructive vein lesions should be treated immediately by venoplasty and stenting to prevent early reocclusion. However, randomised controlled trails are needed to evaluate the long-term benefit of endovenous thrombolysis in patients with acute DVT. 


\section{References:}

1. Hirsh J, Guyatt G, Albers GW, Harrington R, Schunemann HJ. Executive summary: American College of Chest Physicians Evidence-Based Clinical Practice Guidelines (8th Edition). Chest. 2008 Jun;133(6 Suppl):71S-109S.

2. Meissner MH, Manzo RA, Bergelin RO, Markel A, Strandness DE, Jr. Deep venous insufficiency: the relationship between lysis and subsequent reflux. J Vasc Surg. 1993 Oct;18(4):596-605; discussion 6-8.

3. Singh H, Masuda EM. Comparing short-term outcomes of femoral-popliteal and iliofemoral deep venous thrombosis: early lysis and development of reflux. Ann Vasc Surg. 2005 Jan;19(1):74-9.

4. Pianta MJ, Thomson KR. Catheter-Directed Thrombolysis of Lower Limb Thrombosis. Cardiovasc Intervent Radiol. 2010 May 12.

5. Motarjeme A. Ultrasound-enhanced Thrombolysis. J Endovasc Ther. 2007 Apr;14(2):251-6.

6. Parikh S, Motarjeme A, McNamara T, Raabe R, Hagspiel K, Benenati JF, et al. Ultrasoundaccelerated Thrombolysis for the Treatment of Deep Vein Thrombosis: Initial Clinical Experience. Journal of Vascular and Interventional Radiology. 2008;19(4):521-8.

7. Mewissen MW, Seabrook GR, Meissner MH, Cynamon J, Labropoulos N, Haughton SH. Catheterdirected thrombolysis for lower extremity deep venous thrombosis: report of a national multicenter registry. Radiology. 1999 Apr;211(1):3949.

8. Breuss JM, Gallo J, DeLisser HM, Klimanskaya IV, Folkesson HG, Pittet JF, et al. Expression of the beta 6 integrin subunit in development, neoplasia and tissue repair suggests a role in epithelial remodeling. J Cell Sci. 1995 Jun;108 ( Pt 6):224151.

9. Braaten JV, Goss RA, Francis CW. Ultrasound reversibly disaggregates fibrin fibers. Thromb Haemost. 1997 Sep;78(3):1063-8.

10. Chamsuddin A, Nazzal L, Kang B, Best I, Peters G, Panah S, et al. Catheter directed thrombolysis with the Endowave system in the treatment of acute massive pulmonary embolism: a retrospective multicenter case series. J Vasc Interv Radiol. 2008 Mar;19(3):372-6.

11. The Interventional Management of Stroke (IMS) II Study. Stroke. 2007 Jul;38(7):2127-35.

12. Tsivgoulis G, Eggers J, Ribo M, Perren F, Saqqur M, Rubiera M, et al. Safety and efficacy of ultrasound-enhanced thrombolysis: a comprehensive review and meta-analysis of randomized and nonrandomized studies. Stroke. 2010 Feb;41(2):280-7.

13. Alesh I, Kayali F, Stein PD. Catheter-directed thrombolysis (intrathrombus injection) in treatment of deep venous thrombosis: a systematic review. Catheter Cardiovasc Interv. 2007 Jul 1;70(1):143-8.

14. Mewissen MW. Catheter-directed thrombolysis for lower extremity deep vein thrombosis. Tech Vasc Interv Radiol. 2001 Jun;4(2):111-4.

15. Elsharawy M, Elzayat E. Early results of thrombolysis vs anticoagulation in iliofemoral venous thrombosis. A randomised clinical trial. Eur J Vasc Endovasc Surg. 2002 Sep;24(3):209-14.

16. Awad AS, Rouse M, Huang L, Vergis AL, Reutershan J, Cathro HP, et al. Compartmentalization of neutrophils in the kidney and lung following acute ischemic kidney injury. Kidney Int. 2009;75(7):689-98.

17. Comerota AJ, Throm RC, Mathias SD, Haughton S, Mewissen M. Catheter directed thrombolysis for iliofemoral deep venous thrombosis improves health related quality of life. J Vasc Surg. 2000 Jul;32(1):130-7.

18. Comerota AJ. Quality-of-life improvement using thrombolytic therapy for iliofemoral deep venous thrombosis. Rev Cardiovasc Med. 2002;3 Suppl 2:S61-7.

19. Baekgaard N, Broholm R, Just S, Jorgensen M, Jensen LP. Long-term results using catheterdirected thrombolysis in 103 lower limbs with acute iliofemoral venous thrombosis. Eur J Vasc Endovasc Surg. 2010 Jan;39(1):112-7. 


\section{CHAPTER 8}

\section{Ultrasound Accelerated Catheter Di- rected Thrombolysis in acute Ili- ofemoral deep venous thrombosis}

Rob H.W. Strijkers

Jochen. Grommes

Carsten W.K.P. Arnoldussen

Rick de Graaf

Arina J. ten Cate Hoek

Cees H.A. Wittens

Journal of vascular surgery, venous and lymphatic disease, 2013Vol. 1, Issue 3, p225-230 


\begin{abstract}
:
Background: Iliofemoral Deep Vein Thrombosis (DVT) is associated with a high incidence of the post-thrombotic syndrome. The current CHEST guidelines suggest that catheter directed thrombolysis can be used for patients with acute iliofemoral DVT and severe leg complaints. Current literature shows that catheter directed thrombolysis increases patency of the affected tract and may reduce post-thrombotic complications, but treatment time and bleeding complications are high. Ultrasound Accelerated Catheter Directed Thrombolysis (UACDT) uses ultrasound waves to enhance clot lysis, which should lower treatment time and bleeding complications with the same or higher patency rates. We report our clinical experience with UACDT on patency and complications in patients with acute iliofemoral DVT.
\end{abstract}

Methods: Patients treated with UACDT for acute iliofemoral DVT were included in our analyses. Diagnosis of iliofemoral DVT was confirmed using duplex sonography and MR-venography. In addition to thrombolysis, stents were placed or AV-fistula created to ensure patency of the treated vein, if indicated. Main outcome is patency after one year. Secondary outcome measures are: treatment time, bleeding complications, and pulmonary embolism. Patency was assessed using duplex sonography.

Results: In total 37 patients (average age at intervention 42 years, range 5-76 years) were included. The DVT location was unilateral in 33 patients $(20$ left side, 13 right side) and 4 bilateral. Average treatment time was $43 \pm 17$ hours, success rate of thrombolysis was 95\% (n=35), re-thrombosis occurred in 11 $(30 \%)$ patients. Major bleeding occurred in one patient $(3 \%)$ and three minor bleedings occurred at the insertion side of the catheter (8\%). One pulmonary embolism was encountered (3\%). One patient had fever with positive blood cultures for Staphylococcus aureus. Additional procedures were required in $54 \%(n=20)$ of patients. Primary patency was $70 \%$ at one year; Secondary patency was $87 \%$ after one-year follow-up.

Conclusions: Ultrasound accelerated catheter directed thrombolysis of acute iliofemoral DVT is feasible and safe. Supplementary PTA and stenting play an important role in preventing rethrombosis. 


\section{Introduction}

Iliofemoral Deep Venous Thrombosis (DVT) is associated with high post thrombotic morbidity.(1,2) PTS is associated with enhanced healthcare costs and decreased quality of life in patients.(3)

Standard therapy for DVT consists of anticoagulation, compression therapy and mobilization. Anticoagulation prevents clot propagation, decreases the risk of pulmonary embolism and prevents recurrence of DVT. $(4,5)$ However anticoagulation does not actively dissolve the thrombus; the intrinsic fibrinolytic system dissolves the clot. Residual thrombosis is associated with post thrombotic morbidity and it is suggested that early removal of the thrombus is beneficial for the prevention of PTS.(6) Early lysis of clot can also restore patency of the vein and preserve valve function.(7) Catheter-directed thrombolysis (CDT) is used to actively dissolve the clot. Through a multi-side hole catheter, thrombolytic agent is infused directly into the clot, which enhances thrombolysis with less thrombolytic agent.(8)

Adding CDT to the standard therapy increases patency of the iliofemoral tract. However there are still concerns towards treatment time and risk of bleeding complications. New techniques are available to treat iliofemoral DVT. Ultrasound Accelerated Catheter Directed Thrombolysis (UACDT) combines standard CDT with the addition of ultrasound waves. In vitro research has shown that the ultrasound waves influence the fibrin strands and increase uptake of thrombolytic drug in the thrombus. (9-11) However it is unclear, if treatment time with UACDT is shorter than CDT alone in patients with iliofemoral DVT.

Previous studies have shown better complete lysis results with UACDT compared to standard CDT. UACDT has also been used in arterial occlusion and massive PE. Both have shown good treatment results with UACDT. (12-14) In our study we studied patency rates of the venous tract after treatment with UACDT after mid-term follow-up for acute iliofemoral DVT. We also focused on the additional procedures necessary, for the prevention of reocclusion and thus preserving patency in the long term.

\section{Methods}

\section{Patients:}

All consecutive patients who underwent UACDT for acute symptomatic iliofemoral DVTs from October 2008 to January 2012 were identified from a prospective cohort. The patient records were reviewed for demographics, indication for treatment, symptoms, DVT risk factors, adjunctive interventions, peri- 
procedural complications, clinical outcomes, and follow-up with duplex ultrasound (DU) imaging and MR imaging.

The age of the thrombus was assessed by the duration of patient complaints at presentation. If complaints were existent 21 days or less, the thrombus was considered young. Patients, who had complaints for more than 21 days, were considered to have an old thrombus. These patients were excluded from our analysis. Patients with stent thrombosis or thrombosis of the arm were also excluded from this analysis.

Before treatment with UACDT, patients were screened for contra-indications and high bleeding risk as previously described by Grommes et al. doi:10.1016/j.ejvs.2010.11.035.(15) Patients received routinely MRVenography to assess the venous tract on any underlying disease. Blood analysis for genetic clotting disorders was not routinely performed and only carried out on indication.

\section{Thrombolysis}

The interventional radiologist placed the ultrasound accelerated CDT catheter under phlebographic control. The EKOS® EndowaveTM Peripheral Lysis System (EKOS Corporation, Bothell, WA, USA) consists of a multi-lumen infusion catheter with removable, coaxial Ultrasound (US) core and a control unit that simultaneously delivers high-frequency (2.2 MHz), low-energy (0.45 W) US energy and thrombolytic drug into the thrombus. All patients received UACDT with the EKOS endowave system. For thrombolytic agent we used rt-PA in Aachen and Urokinase in Maastricht. Thrombolysis with rt-PA was given $1 \mathrm{mg} /$ hour with a single bolus of $5 \mathrm{mg}$. Urokinase was given with 100.000 units/hour with a single bolus of 250.000 units. In case of bilateral DVT, two catheters were inserted and the drug dosage was evenly divided between the two catheters. During thrombolysis we monitored Fibrinogen levels, Hemoglobin levels (Hb), activated partial thromboplastin time(APTT), and prothrombin time standardized to international normalized ratio (INR). Additionally all patients received standard anticoagulation treatment according to the ACCP guidelines.(4) During active thrombolysis, anticoagulant treatment was substituted by intravenous Unfractionated Heparin guided on APTT. Inferior vena cava filters are not used routinely in our hospital. None of our patients received a caval filter. After thrombolysis patients resumed their standard DVT therapy with oral anticoagulation, compressive stockings and mobilization according to the ACCP guidelines.(4) 


\section{Additional procedures}

After successful CDT procedures, patients received additional interventions if indicated by venography. Additional procedures were performed immediately after successful thrombolysis. Stenting was not used as a secondary salvage procedure. The interventional radiologist or vascular surgeon performed additional procedures such as, percutaneous transluminal angioplasty with or without stenting, construction of an AV-fistula or other open procedures to increase inflow. We used self-expandable stents for stenting for underlying obstructions (sinus-XL® from Optimed and Zilver vena ${ }^{\mathrm{TM}}$ from Cook®). Table 1 shows the patient characteristics of the group.

\begin{tabular}{|l|l|l|l|}
\hline Patient characteristics $(\mathbf{N}=37)$ & Aachen $=20$ & \\
\hline Center & Maastricht $=17$ & Min $=5$ years & Max $=76$ years \\
\hline Age & AVG $=42$ years & Female $=19$ & \\
\hline Gender & Male $=18$ & Right $=13$ & Bilateral $=4$ \\
\hline DVT side & Left $=20$ & lliofemoral $=32$ & IVC $=4$ \\
\hline Thrombus stage & Femoro-popliteal $=1$ & \\
\hline Thrombus age & 0-6days $=5$ & $7-13$ days $=21$ & $14-20$ days $=11$ \\
\hline
\end{tabular}

\section{Definitions}

Clot load was defined with the scoring system used in the national venous registry.(16) A thrombus score was calculated for seven venous segments: the IVC, the common iliac vein, the external iliac vein, the common femoral vein, the proximal portion of the superficial femoral vein, the distal portion of the superficial femoral vein, and the popliteal vein. The thrombus score was 0 when the vein was patent and completely free of thrombus, 1 when partially occluded, and 2 when completely occluded. The pretreatment score was substracted by the post treatment score and then divided by the original clotload, which resulted in determination of the lysisgrade. Success of thrombolysis was divided in three categories: Grade 1: Complete clot lysis defined as > $90 \%$ lysis (restored patency), and grade 2 partial clot lysis defined as 50-90\% lysis (restored patency) Grade 3: $<50 \%$ lysis. Assessment of the post treatment clot load was made purely on the final phlebography before additional procedures 
were done. If there was no recanalization at all, this was considered as treatment failure.

\section{Bleeding}

Bleeding was classified as major, if it was overt with a fall in haemoglobin of $\geq 2$ $\mathrm{g} / \mathrm{dl}$, or when hemorrhage led to transfusion of 2 units of packed red blood cells (RBCs) or whole blood. Bleeding situated in a critical organ (intra-cranial, retroperitoneal or pericardial) or, if it contributed to death, was also defined as a major bleeding. Bleeding was classified as minor, if it was situated near the catheter-insertion site.

\section{Follow-up}

During follow-up visits all patients were assessed routinely with duplex ultrasound. At follow-up the venous tract was considered patent or not patent. Recurrent DVT, pulmonary embolism (PE), major bleeding, retreatment and or other complications were registered. Duplex ultrasound of the venous tract was performed by a dedicated sonographer and the complete venous tract was routinely assessed. Follow-up visits were planned according to our standard care at 6-8 weeks after stent placement, 3 months, 6 months and one year. If the patient experienced any complaints follow-up visits were planned at the earliest convenience. PE was assessed on patient's symptomatology and confirmed with CT-angiography.

\section{Statistical analysis}

Categorical data are presented as frequencies and percentages, and continuous data are presented as mean values \pm the standard deviation (SD) or median values with the range, unless otherwise stated. Cumulative probabilities of patency at 3, 6 and 12 months after initial treatment are calculated using Kaplan Meier survival analysis. For the evaluation of primary patency, the need for additional treatment by either an assisted primary intervention or a secondary intervention or obstruction at the end of follow up is considered as treatment failures. For the evaluation of secondary patency only obstruction at the end of follow-up was considered a treatment failure. Follow up time was defined as the period between initial treatment and treatment failure or the period between initial treatment and date of last observation (in patients without treatment failure). Analyses were carried out with commercially available statistical programs: SPSS version 17 (SPSS version 17.0.1; SPSS, Chicago, IL) and STATA (STATA version 11.2). P values $\leq .05$ were considered to indicate statistical significance 


\section{Results}

\section{Treatment success}

Immediate treatment success with UACDT showed that grade 1 lysis was achieved in 19 of the 37 patients (51\%). Grade 2 lysis was achieved in 16 of the 37 patients (43\%). Average thrombolysis time was 43 hours \pm 17 . Two patients (5\%) had an occluded segment without any signs of recanalization after 72 hours and thrombolysis was stopped at that time. The clot load per individual patient pre- and post-thrombolysis is shown in figure 1.
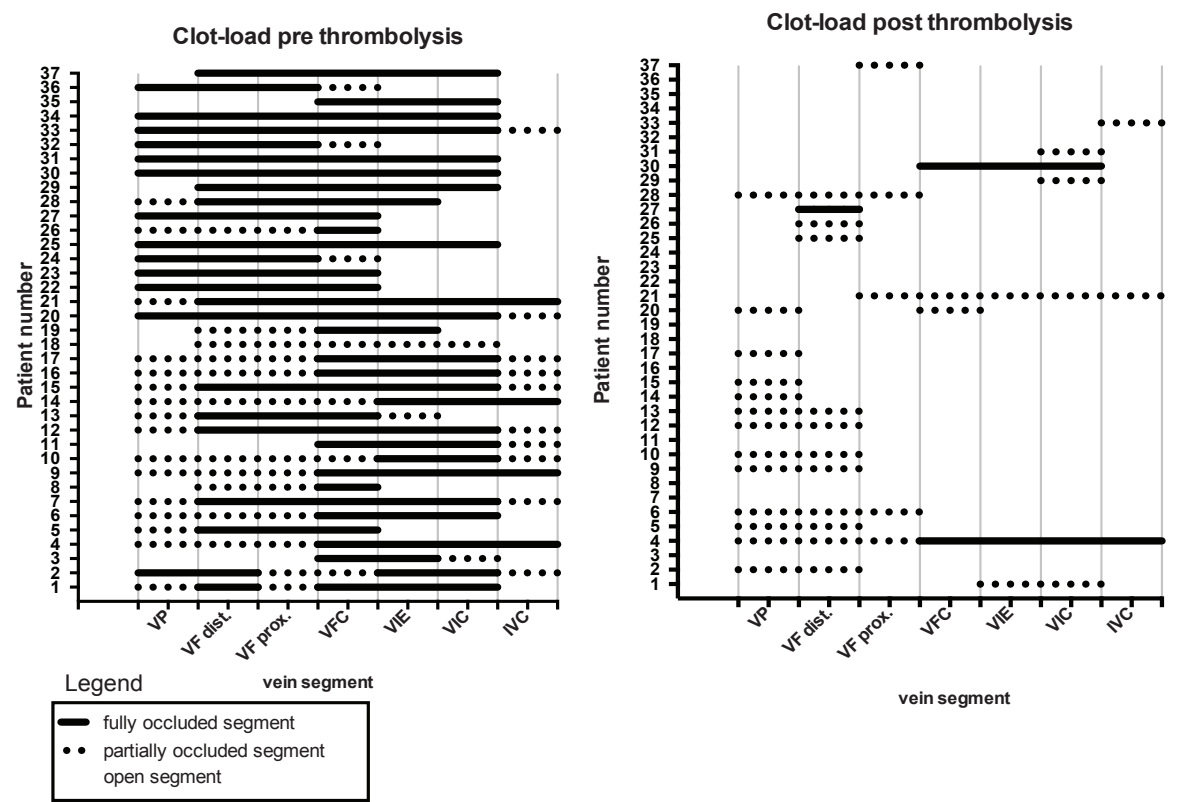

Twenty patients initially presented with a left-sided DVT, 15 (75\%) of them had May Thurner syndrome. Two patients $(10 \%)$ had an inferior caval vein atresia, one patient had another form of compression syndrome and two patients had no anatomical variance.

In total 20 patients (54\%) received an additional procedure. Additional stenting was performed in 15 patients (41\%), 3 patients (8\%) had an open intervention of which in 2 patients an arterial-venous fistula was created and one patient had a surgical thrombectomy. Two patients (5\%) received additional PTA of the common iliac vein. We observed no pulmonary embolism during UACDT treatment. Table 2 shows the additional treatments with their respective indications. 


\begin{tabular}{|l|c|l|}
\hline Additional treatment & Palients & Indication (number of patients) \\
\hline PTA & 2 & Post thrombotic trabeculae (2) \\
\hline PTA + Stenting & 15 & $\begin{array}{l}\text { May thurner syndrome (11), vena cava } \\
\text { atresia (3), other compression } \\
\text { syndrome (1) }\end{array}$ \\
\hline AV-fistula & 2 & Insufficient inflow (2) \\
\hline Surgical thrombectomy & 1 & Early rethrombosis (1) \\
\hline
\end{tabular}

\section{Follow-up}

In the early follow-up we found that 11 patients (30\%) had a re-thrombosis. Most recurrent thrombosis occurred in the first two weeks after the initial procedure. Nine of these patients have undergone additional procedures to regain a patent venous tract. Three of the patients had a rethrombosis due to failed anticoagulation of which two patients had a Heparin induced thrombocytopenia. These patients were switched to other anticoagulation therapy. The six other patients had a rethrombosis due to failed stent placement or delay in stent placement.

Primary patency is $70 \%$ (number of patients at risk: 14) at one-year follow-up. Secondary patency is $87 \%$ at one-year follow-up (number of patients at risk: 16). Figure 2 shows the Kaplan Meier plot illustration of the estimated percentage of patients with patency at one year of follow-up. 
Cumulative probability of primary and secondary patency

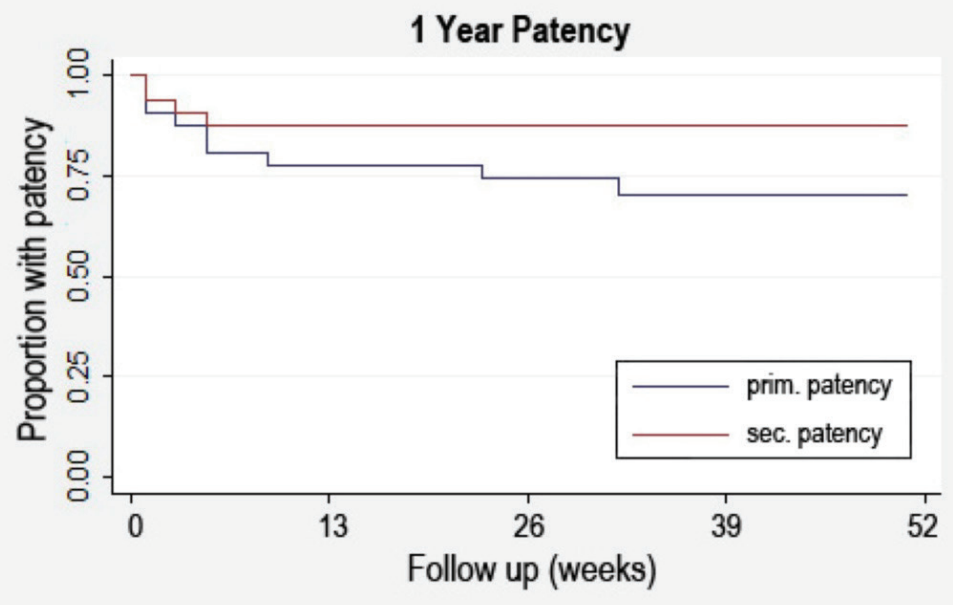

Number at risk (Std. Error)

\begin{tabular}{|llllll|}
\hline Weeks & 0 & 13 & 26 & 39 & 52 \\
Primary patency & 32 & $23(0.07)$ & $21(0.08)$ & $18(0.08)$ & $14(0.08)$ \\
Secundary patency & 33 & $25(0.06)$ & $22(0.06)$ & $20(0.06)$ & $16(0.06)$ \\
\hline
\end{tabular}

Figure 2. Cumulative incidence of main outcomes according to primary and secondary patency after one-year follow-up. Primary patency at 52 weeks 0.7039, Standard error [95\% confidence interval] 0.0835 [0.5061-0.8344]. Secundary patency at 52 weeks 0.8746 , standard error [95\% Confidence interval] 0.0587 [0.6992-0.9511]

\section{Complications}

In three cases a minor bleeding at the catheter insertion side was detected. In one case ( $3 \%$ ) there was also a bleeding observed at the insertion side of the catheter, at the time of detection the patient required blood transfusion with two packed cells, and this was considered a major bleeding. One patient developed fever with positive blood cultures for Staphylococcus aureus due to prolonged catheterization. This patient was treated with antibiotics for six weeks and fully recovered. One patient suffered from an aortic aneurysm for which he needed acute surgical repair. This event was considered to be unrelated to the UACDT procedure. One PE was encountered (3\%) two weeks after initial UACDT treatment caused by Heparin Induced Thrombocytopenia (HIT). Overall the incidence of major bleeding and PE is low. 


\section{Discussion}

The current standard for the treatment of iliofemoral DVT consists of anticoagulation therapy with additional compression therapy and mobilization. Recently the new ACCP guidelines suggested CDT for patients with iliofemoral DVT in order to decrease post thrombotic morbidity. In addition the recently published CaVenT-study showed that CDT in patients with iliofemoral DVT decreases the absolute risk of PTS with 14\%. Currently two trials are still ongoing (the US Attract-trial and the Dutch CAVA-trial). The Attract trial studies the effects of early thrombus removal in patients with iliofemoral DVT.(17) Our own group is currently conducting the CAVA-trial, which studies the effect of additional UACDT in patients with acute iliofemoral DVT. The results of these two ongoing trials will be important to provide additional evidence on the effectiveness of acute thrombus removal for the reduction of the incidence of PTS in the long term. Potentially severe complications such as intracranial hemorrhage and intraperitoneal bleeding are the main reason that CDT is still not considered standard treatment. Our data shows that the risk of major bleeding is low. Techniques such as UACDT may further decrease complication rates of thrombus removal treatment compared to the standard CDT treatment.

UACDT has shown short treatment times in small cohort studies. In our study the median treatment time is under two days (43 hours or 1.8 days). This is longer than previously reported by Motarjeme and Raabe.(11,13) This could be due to a number of factors such as differences in patient selection, treatment procedures etc. More research is needed to determine the optimal patient selection and treatment strategy. Comparison of clot burden proves difficult between studies. This is why we like to emphasize the need for standardized reporting on thrombus location and extent in DVT. This will enable a good comparison between studies and make future pooling of the gathered data possible. We suggest the use of the LOVE and LET score for future reports on lower extremity venous thrombo-embolic disease. $(18,19)$ Another possible reason for a longer clot-lysis time in our series is related to clot age. The average clot age treated in the CaVenT-study was 6 days. The clots we encountered in our patient population were older with most patients presenting after 7-13 days. Older clots are thought to be more difficult to lyse. This emphasizes the need for optimal patient selection.

Our study shows safety and feasibility of UACDT in patients with acute iliofemoral DVT using the EKOS endowave system. In about almost $50 \%$ of all patients an additional treatment with stenting and/or AV-fistula was performed. In most cases May-Thurner syndrome was present and obstructed the outflow on the left side. In our previously reported experience we commented on early rethrombosis in patients with delayed treatment of an underlying obstruc- 
tion.(15) Again in this cohort we observed frequent early rethrombosis occurring in 11 patients. Nine of these patients had a re-intervention, which finally led to a patent venous tract. In most cases delay in stent placement was considered to be the main reason for early rethrombosis. We recommend stent placement immediately after successful thrombolysis. In three cases we witnessed failure of anticoagulation, one failure in administration of the drug and in two cases the patient had a Heparin induced thrombocytopenia. Compared to the study of Baekgaard our stenting percentages are comparable.(20) These observations support the idea that, if indicated, immediate stenting might prevent early re-thrombosis. Diagnostic imaging plays a crucial role in identifying these underlying obstructions. In our study in both centers we used MRvenography combined with duplex sonography routinely in all patients. These imaging techniques were used to identify underlying stenosis and decide on the treatment options. We experienced technical problems with the stents designed for arterial use, when used in the venous system, which in one case led to occlusion of the vein. Future dedicated venous stents will hopefully prevent this kind of technical failure.

Although we managed to achieve good secondary patency of the venous tract, this does not provide us with information on the clinical outcome for the incidence of PTS or impact of patency on the quality of life in these patients. Future studies should therefore preferably incorporate clinical relevant outcomes such as PTS and Quality of life.

\section{Conclusion}

UACDT is feasible and safe with excellent secondary patency. Adequate patient selection, dedicated diagnostic imaging and early stenting seem to play an important role in the prevention of early rethrombosis. 


\section{References:}

1. Akesson H, Brudin L, Dahlström JA, Eklof B, Ohlin P, Plate G. Venous function assessed during a 5 year period after acute ilio-femoral venous thrombosis treated with anticoagulation. Eur J Vasc Surg. 1990Feb.;4(1):43-8.

2. Kahn SR, Shrier I, Julian JA, Ducruet T, Arsenault L, Miron MJ, et al. Determinants and time course of the postthrombotic syndrome after acute deep venous thrombosis. Ann. Intern. Med. 2008Nov.18;149(10):698-707.

3. Ashrani AA, Heit JA. Incidence and cost burden of post-thrombotic syndrome. J Thromb Thrombolysis. 2009Nov.;28(4):465-76.

4. Kearon C, Kahn SR, Agnelli GG, Goldhaber S, Raskob GE, Comerota AJ. Antithrombotic Therapy for Venous Thromboembolic Disease: American College of Chest Physicians Evidence-Based Clinical Practice Guidelines (8th Edition). Chest. 2008Jun.1;133(6 suppl):454S-545S.

5. Hull R, Hyers T, Prins M, Raskob G. Antithrombotic therapy for venous thromboembolic disease. Chest. 2004.

6. Comerota AJ, Grewal N, Martinez JT, Chen JT, Disalle R, Andrews L, et al. Postthrombotic morbidity correlates with residual thrombus following catheter-directed thrombolysis for iliofemoral deep vein thrombosis. Journal of Vascular Surgery. 2012Jan.24.

7. Meissner MH, Manzo RA, Bergelin RO, Markel A, Strandness DE. Deep venous insufficiency: the relationship between lysis and subsequent reflux. Journal of Vascular Surgery. 19930ct.;18(4):596-605; discussion606-8.

8. Baldwin ZK, Comerota AJ, Schwartz LB. Catheter-directed thrombolysis for deep venous thrombosis. Vascular and Endovascular Surgery. 2004;38(1):1-9.

9. Siddiqi F, Blinc A, Braaten J, Francis CW. Ultrasound increases flow through fibring gels. Thromb Haemost. Schattauer; 1995;73(3):495-8.

10. Francis CW, Blinc A, Lee S, Cox C. Ultrasound accelerates transport of recombinant tissue plasminogen activator into clots. Ultrasound Med Biol. 1995;21(3):419-24.

11. Motarjeme A. Ultrasound-enhanced Thrombolysis. J. Endovasc. Ther. 2007Apr.;14(2):251-6.

12. Parikh S, Motarjeme A, McNamara T, Raabe R, Hagspiel K, Benenati JF, et al. Ultrasoundaccelerated Thrombolysis for the Treatment of Deep Vein Thrombosis: Initial Clinical Experience. Journal of Vascular and Interventional Radiology. 2008Apr.;19(4):521-8.

13. Raabe RD. Ultrasound-accelerated Thrombolysis in Arterial and Venous Peripheral Occlusions: Fibrinogen Level Effects. JVIR. Elsevier Inc; 2010Aug.1;21(8):1165-72.

14. Lin PH, Zhou W, Dardik A, Mussa F, Kougias P, Hedayati N, et al. Catheter-direct thrombolysis versus pharmacomechanical thrombectomy for treatment of symptomatic lower extremity deep venous thrombosis. The American Journal of Surgery. 2006Dec.;192(6):782-8.

15. Grommes J, Strijkers RHW, Greiner A, Mahnken AH, Wittens CHA. Safety and feasibility of ultrasound-accelerated catheter-directed thrombolysis in deep vein thrombosis. European Journal of Vascular \& Endovascular Surgery. 2011Apr.;41(4):526-32.

16. Mewissen MW, Seabrook GR, Meissner MH, Cynamon J, Labropoulos N, Haughton SH. Catheterdirected thrombolysis for lower extremity deep venous thrombosis: report of a national multicenter registry. Radiology. 1999Apr.;211(1):39-49.

17. Comerota AJ. The ATTRACT Trial: Rationale for Early Intervention for Iliofemoral DVT. Perspectives in Vascular Surgery and Endovascular Therapy. 2010Jul.13;21(4):221-5.

18. Arnoldussen CWKP, Toonder I, Wittens CHA. A novel scoring system for lower-extremity venous pathology analysed using magnetic resonance venography and duplex ultrasound. Phlebology. 2012;27 Suppl 1:163-70.

19. Arnoldussen CWKP, Wittens CHA. An imaging approach to deep vein thrombosis and the lower extremity thrombosis classification. Phlebology. 2012;27 Suppl 1:143-8.

20. Baekgaard N, Broholm R, Just S, Joslashrgensen M, Jensen LP. Long-Term Results using Catheter-directed Thrombolysis in 103 Lower Limbs with Acute Iliofemoral Venous Thrombosis. European Journal of Vascular \& Endovascular Surgery. Elsevier Ltd; 2010Jan.1;39(1):112-7. 


\section{CHAPTER 9}

\section{Venous in-stent thrombosis treated by ultrasound accelerated catheter directed thrombolysis}

Short title: Venous in-stent thrombosis treated by thrombolysis

Rob H.W. Strijkers

Mark A.F. de Wolf

Carsten W.K.P. Arnoldussen

Milea J.M. Timbergen

Rick de Graaf

Arina J. Ten Cate-Hoek

Cees H.A. Wittens

European Journal of Vascular and Endovascular Surgery (2015) 49, 440e447 


\begin{abstract}
Introduction: Stent placement in the venous system is an increasingly used treatment modality in chronic venous obstruction and as additional treatment after thrombolytic therapy in iliofemoral DVT. We report on our experience in treating in-stent thrombosis with Ultrasound Accelerated Catheter Directed Thrombolysis (UACDT).
\end{abstract}

Methods: Retrospective analysis of patients treated for venous stent occlusion, after Percutaneous Transluminal Angioplasty (PTA) and stent placement for either chronic venous occlusive disease or persistent vein compression in patients with acute DVT. Duration of occlusion and suspected clot age were assessed using patient complaints and typical findings on duplex ultrasonography (DUS). DUS and venography were used to assess patency and to determine the cause of reocclusion. Acute treatment of occlusion consisted of UACDT. Additional procedures included: PTA, stent placement, and creation of an arteriovenous-fistula.

Results: We identified 18 patients, who were treated for occluded stent tracts with UACDT between January 2009 and July 2014. Median age was 43 years, and $67 \%$ were male. Indications for initial stenting were; treatment of chronic venous obstructive disease in 12 patients and treatment of underlying obstruction after initial thrombolysis in acute DVT in 6 patients. Technical success was achieved in 61\% (11/18). Primary patency at last follow-up was 73\% (8/11), during median follow-up of 14 months (0-41). Additional treatments after successful lysis were; restenting in 7 patients, and creation of an arteriovenousfistula in 6 patients

Conclusion: Treatment with ultrasound-accelerated catheter directed thrombolysis of occluded stent tracts is feasible and effective.. Recanalization of the stent tract can be achieved in most cases. Additional interventions were frequently used after successful UACDT treatment. Most stent occlusions were caused by stent related problems. 


\section{Introduction}

During the last two decades endovenous recanalization, by percutaneous transluminal angioplasty (PTA) and stenting, in chronic deep venous occlusive disease has quickly gained in popularity. Many authors have shown excellent clinical success rates. ${ }^{1-3}$ Because of this, and as stenting seems to be the only treatment aimed at resolving the underlying pathology in post-thrombotic syndrome (PTS) and iliac vein compression syndromes, it has been implemented in many expert-venous centers worldwide as a first-line treatment. Complication rates have been shown to be mild, with generally no clinically relevant pulmonary embolisms and no procedure related mortality. ${ }^{3-5}$ The most important complication is acute reocclusion of the stented venous segments, with or without thrombus propagation into proximal or distal vein tracts. This occurs in $20-30 \%$ of cases, even when adequate anticoagulation regimens are used. ${ }^{4}$ In cases of stent re-occlusion return of signs and symptoms of venous disease can be expected, and in some cases even worsening of complaints compared to the pre-recanalization state. An important step in preventing reocclusion is to guarantee adequate in- and outflow for the stents. Inflow problems can arise when veins caudal to the stented segments are also involved in the post-thrombotic process, most notably the femoral and deep femoral vein, as flow volume in those cases might not be sufficient to maintain patency. Outflow problems arise when venous tracts cephalad to the stents are (partly) occluded due to endoluminal pathology or external compression. Furthermore in-stent problems, for example residual compression by overlying arteries or stenosis, kinking or fractures are also thought to lead to higher loss of patency. Prevention by extending stents cephalad or caudal into a healthy vein tract and using stents that withstand the pressure from overlying structures such as arteries and do not kink are therefore very important. ${ }^{6}$ Adequate antithrombotic therapy is also important in preventing rethrombosis.

In cases where reocclusion does occur removal of as much of the thrombus load as quickly as possible is generally thought necessary. ${ }^{7}$ One possible option is to remove the thrombus, by means of pharmaco-mechanical thrombolysis. Ultrasound accelerated catheter directed thrombolysis (UACDT) is used for acute occlusions in the arterial system, DVT and pulmonary embolism. ${ }^{8-11}$ Literature shows good safety and feasibility for UACDT for these indications. Possible advantages of UACDT over the use of a normal thrombolysis catheter are: shortened treatment duration and less thrombolytics used reducing the risk of (major) bleeding.12 With adequate patient selection, the chance of gaining technical success is around $90 \%$ in reported literature associated with minimal bleeding risk.13 
We report on our experience of treating in-stent thrombosis with UACDT in patients referred to our tertiary center. Furthermore we describe the pitfalls that we have encountered in these cases and provide recommendations how to follow-up on patients after venous stenting.

\section{Methods}

\section{Population:}

From October 2009 till July 2014 all patients treated for venous stent occlusion by UACDT were eligible for retrospective analysis. All patients were evaluated for bleeding risk. Indication for primary stent placement was either chronic venous occlusive disease or persistent vein compression in patients with acute iliofemoral DVT during thrombolytic therapy. Time between stent placement and occlusion was assessed and divided into two groups (within 6 months after stent placement, and longer than 6 months after stent placement). Occlusion side was noted and if available thrombophilia status was reported. No additional testing for thrombophilia factors was performed. Duration of the occlusion and suspected clot age were assessed using patient complaints and findings on duplex ultrasonography (DUS). ${ }^{14}$ Patient complaints were assessed and used as the criterion to categorize patients into two groups; 21 days or shorter interval of complaints and complaints for over 21 days. The last patent duplex scan was taken as a reference value for assessment of clot age. Stent occlusion was diagnosed using DUS with normal B-mode settings, power-flow, e-flow, conventional color and pulse wave Doppler to assess flow in the stent tract. We used at least one of the available modalities or combinations. The venous tract was assessed in both transverse and longitudinal planes. In order to visualize flow in the stented ilio-caval tract a number of flow augmenting maneuvers were performed such as inspiration and expiration, dorsiflexion of the foot, and gluteal muscle contraction.

Before recanalization and catheter placement, venography was routinely performed, to confirm the diagnosis of stent occlusion.

Dutch law allows for retrospective analysis of patient data without specific approval of an ethical committee.

\section{Intervention:}

The interventional radiologist placed the UACDT catheter under venographic control. The EKOS® EndowaveTM Peripheral Lysis System (BTG International Ltd, L) consists of a multi-lumen infusion catheter with removable, coaxial Ultrasound (US) cores and a control unit that simultaneously delivers highfrequency (2.2 MHz), low-energy (0.45 W) US energy and thrombolytic drug 
into the thrombus. All patients received UACDT with the EKOS Endowave system. Urokinase (Medacinase $\AA$ Lamepro the Netherlands) was in all cases used as the thrombolytic agent. Urokinase was administered with $100 \quad 000$ units/hour after a single bolus of 250000 units at the start of treatment. In cases of bilateral occlusion, two catheters were inserted and the drug dosage was evenly divided over the two catheters, each with their own EKOS machine. During thrombolysis fibrinogen levels, Hemoglobin levels (Hb), activated partial thromboplastin time (APTT), prothrombin time standardized to international normalized ratio (INR), thrombocytes and D-dimer levels were routinely monitored. During active thrombolysis, anticoagulant treatment was substituted by intravenous unfractionated heparin guided by APTT (between 40-60 seconds or 1.2-1.7 times the reference value of 34 seconds). At the same time patients were fitted with intermittent pneumatic compression sleeves. Inferior vena cava filters are not used routinely in our hospital. None of our patients received a caval filter. After thrombolysis, patients either resumed or started oral anticoagulation for at least 6 months or, if indicated lifelong (recurrent DVT). Adequate anticoagulation was achieved with Coumadin with an International normalized ratio (INR) between 2.5 and 3.5 according to the standards of the Dutch federation of thrombosis services (FNT) or Rivaroxaban 20mg once daily. The choice of anticoagulant agent was based on patient preference. Compressive stockings were routinely offered to patients and worn based on preference of the patient. Mobilization was encouraged as much as possible.

\section{Assessment of recanalization:}

Phlebographic control was performed on a daily basis to monitor thrombolysis progression and to evaluate obstructions in the venous outflow tract. Duration of thrombolysis was determined by assessment of phlebographic imaging and the point where $>90 \%$ lysis of the clot was achieved. ${ }^{15}$ Thrombolysis was continued in a number of cases, because of bridging towards additional treatment. We tried to determine the cause of the stent thrombosis by means of phlebography from multiple angles. In some cases cone-beam CT was used to create a $3 \mathrm{D}$ reconstruction of the stent tract. If the reason for thrombosis of the tract was identified, adequate measures were taken to resolve the problem and prevent future rethrombosis. Objective identification of the underlying cause of stent thrombosis was only possible in patients with successful thrombolysis.

\section{Additional procedures}

After successful UACDT procedures, patients received additional interventions if indicated. Additional procedures were performed as soon as possible after successful thrombolysis. Additional procedures such as, percutaneous transluminal angioplasty (PTA) with or without re-stenting were performed by the 
interventional radiologist. Surgical procedures to increase inflow such as construction of an AV-fistula between the common femoral vein and artery or endophlebectomy were performed by the vascular surgeon. We used selfexpandable stents for stenting for underlying obstructions (sinus-XL $\AA$ and sinus-Venous ${ }^{\circledR}$ from Optimed and Zilver vena ${ }^{\mathrm{TM}}$ from Cook®). Various diameters and lengths stents were used ranging from 12 to $26 \mathrm{~mm}$ in diameter and $60-150 \mathrm{~mm}$ in length. Stent sizes were determined by multiplanar venography.

\section{Complications}

Complications following thrombolysis and follow-up were categorized into groups. These included bleeding complications, pulmonary embolism (PE), recurrent thrombosis, infections and others. Bleeding was classified as major, if it was overt with a fall in hemoglobin of $\geq 2 \mathrm{~g} / \mathrm{dl}$, or when hemorrhage led to transfusion of 2 units of packed red blood cells (RBCs) or whole blood. Bleeding situated in a critical organ (intra-cranial, retroperitoneal or pericardial) or, if it contributed to death, was also defined as a major bleeding. Bleeding was classified as minor, if it was situated near the catheter-insertion site.

\section{Follow-up}

Standardized follow-up was performed. Patients returned at 2 weeks, 6 weeks, 3 months, 6 months, 1 year, and yearly thereafter. If the patient experienced complaints, follow-up was performed at the earliest convenience, usually the same or the next day. During follow-up patency of the stent tract was assessed using DUS. A dedicated sonographer performed DUS. Recurrence, retreatment, $\mathrm{PE}$, and other complications were recorded.

We standardized routine follow-up after stenting in this manner: the day after stenting, patients were checked with DUS to ensure patency of the tract prior to discharge. At 2 weeks, 6 weeks, 3 months and 6 months after stenting the patients were seen with clinical follow-up and routine DUS examination. The complete stented tract was visualized in every patient. Apparent thrombosis and/or lack of flow in the stented tract were used to determine patency.

\section{Results}

\section{Patients}

A total of 19 legs in 18 patients were treated with UACDT for venous stent thrombosis. The majority of patients were male. The average age of the group was 43 years at time of thrombolysis. In 33\% of cases the initial indication for stent placement was to treat an underlying stenosis after acute iliofemoral DVT treatment with UACDT. The remaining $67 \%$ received PTA and stenting as a 
treatment for chronic deep venous obstruction and subsequent complaints. Most stents were placed in the left iliac tract (67\%). We noticed that most stent occlusions occurred within 6 months after initial placement of the stents $(83 \%$ of patients). In $72 \%(n=13)$ of patients we were able to assess the duration of complaints. In the other cases it was not possible to make an indication of duration of complaints, either because of vague complaints or absence of complaints. Eleven patients were assessed as having acute thrombosis (less than 21 days according to the reporting standards in venous disease). Patient characteristics are summarized in table 1.

Before the UACDT to treat the in-stent rethrombosis, patients had received a median of 3 (1-13) stents in 1-3 stenting sessions. This was done on the left side in 13 , on the right side in 1 and bilaterally in 3 cases; one patient only had stents placed in the inferior caval vein. In all patients Nitinol self-expandable stents were used (sinus XL, sinus repo-visual, sinus Venous), in two cases these were combined with Wallstent placement and in one case with balloonexpandable stents (Andramed). 
Table 1 shows the baseline characteristics of the patients.

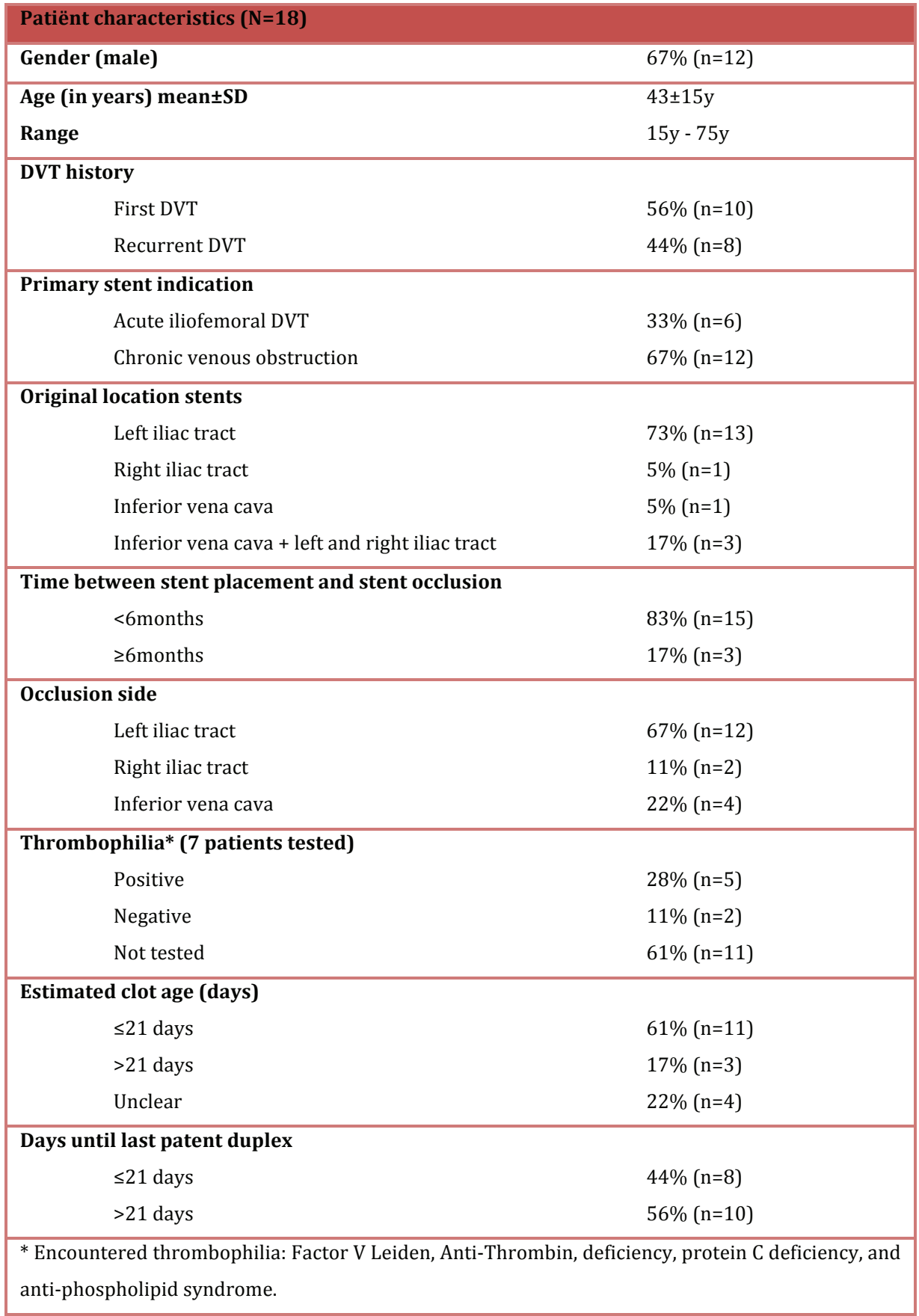




\section{Treatment results and additional procedures}

All patients underwent UACDT with the EKOS catheter. Catheter placement was successful in all cases. In 11 of 18 patients patency was successfully regained. This resulted in a $61 \%$ technical success rate for this procedure. All patients with failed thrombolysis had an unknown or estimated clot age of over 21 days and last patent duplex more than 21 days ago. Median lysis time of successfully lysed cases was 19 hours (range: 11-101). In five cases we performed additional stenting and created an AV-fistula to treat an underlying cause such as stenotic lesions not treated previously. Figure 1 and 2 show pre- and postthrombolysis results. Furthermore we encountered stent related occlusions and insufficient inflow in the stent tract. In two cases we performed additional stenting and in one patient we secured inflow of the stent tract with the creation of an AV-fistula. In total 8/11 (73\%) patients needed ancillary interventions after successful thrombolysis. In all cases of UACDT we identified the underlying causes of rethrombosis. These were; suboptimal positioning, failure due to stent characteristics (kinking of stents and stent fracture), insufficient inflow or inadequate anticoagulation. Figure 3 shows an example of stent fracture in the venous system. Most occlusions we encountered were related to stent failure and suboptimal positioning. Table 2 shows the treatment results. Table 3 shows the time between last patent duplex and occlusion, suspected clot age, and suspected reason for reocclusion.

\section{Figure 1}

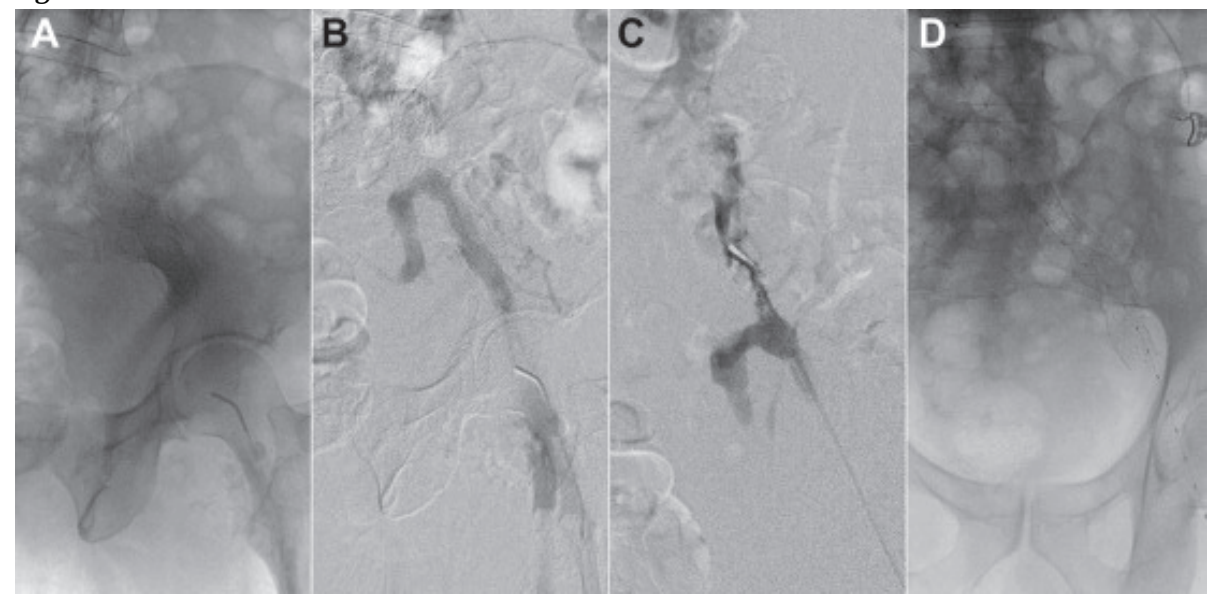

(A)In situ sinus venous stent in the left common iliac vein. (B) Digital subtraction venography with the catheter in the common femoral vein shows contrast filling of the external and internal iliac veins. There is no filling of the (occluded) stent in the common iliac vein. (C) Recanalization of the stent with outflow of contrast to the inferior vena cava. (D) Placement of the 
EKOS Endowave Peripheral Lysis System (BTG International Ltd, London, UK) thrombolysis catheter through the occluded stent.

Figure 2

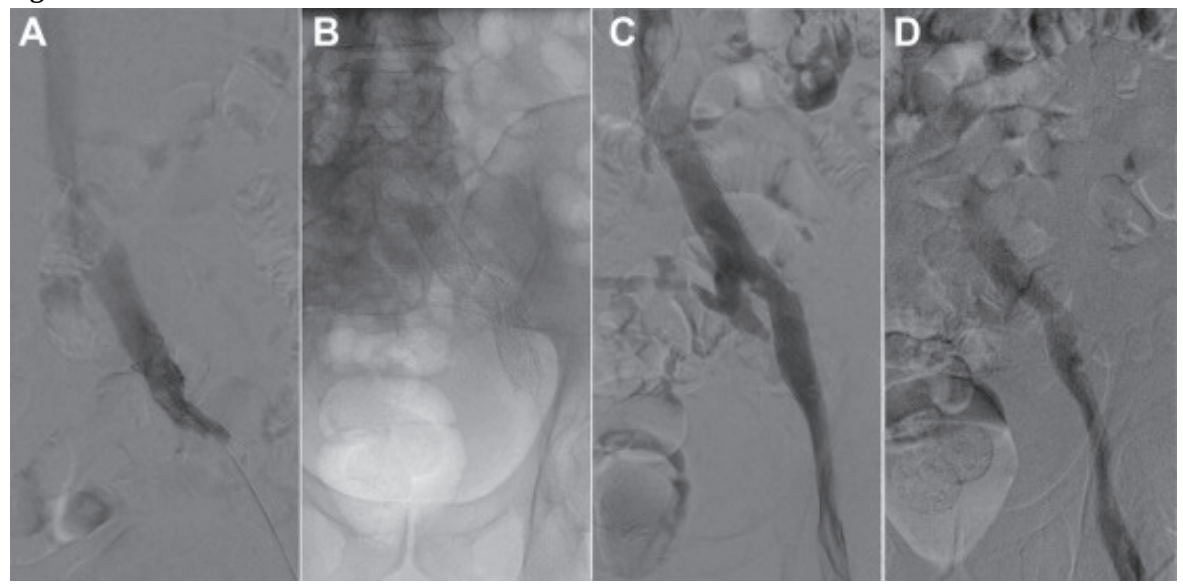

(A)Control venography after extended thrombolysis and balloon angioplasty shows an open lumen with outflow to the inferior vena cava (B) Extension of the stented deep vein tract to the inferior vena cava and external iliac vein to cover the entire deep vein obstruction (C) Control venography after stenting and balloon angioplasty shows improved (out) flow through the iliac veins with persistent filling of pre-sacral collaterals. (D) Final control venography after additional balloon angioplasty shows adequate flow from the groin to the inferior vena cava without filling of collaterals. 


\section{Figure 3}

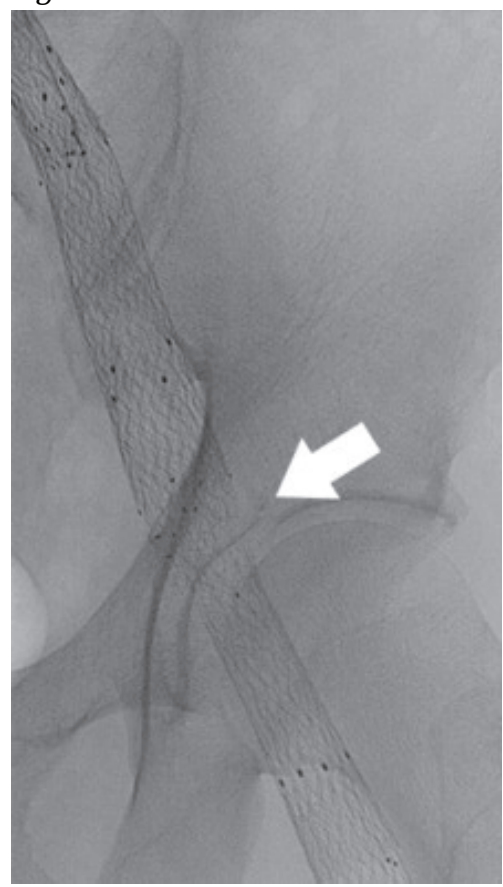

Fractures of the rigid Sinus XL stents in the distal external iliac vein / proximal common femoral vein are seen (arrow).

Table 2 treatment results

\begin{tabular}{|c|c|}
\hline \multicolumn{2}{|l|}{ UACDT procedures $(\mathrm{N}=18)$} \\
\hline $\begin{array}{l}\text { Successful thrombolysis? } \\
\text { Successful } \\
\text { Not successful }\end{array}$ & $\begin{array}{l}61 \%(n=11) \\
39 \%(n=7)\end{array}$ \\
\hline $\begin{array}{l}\text { Duration of successful thrombolysis }(n=11) \\
\text { Median } \\
\text { Range }\end{array}$ & $\begin{array}{l}19 \text { hours } \\
11-101 \\
\text { hours }\end{array}$ \\
\hline Restenting after successful UACDT $(n=11)$ & $64 \%(n=7)$ \\
\hline $\begin{array}{l}\text { Other additional interventions after successful UACDT } \\
(n=11) \\
\text { Arteriovenous fistula }\end{array}$ & $55 \%(n=6)$ \\
\hline
\end{tabular}


Table 3

\begin{tabular}{|c|c|c|c|c|}
\hline $\begin{array}{l}\text { Pa- } \\
\text { tient }\end{array}$ & $\begin{array}{l}\text { Days since last patent } \\
\text { duplex }\end{array}$ & $\begin{array}{l}\text { Suspected slot } \\
\text { age } \\
\leq 21 \text { days } \\
>21 \text { days } \\
\text { unknown }\end{array}$ & $\begin{array}{l}\text { Lysis suc- } \\
\text { cessful } \\
\text { Yes/No }\end{array}$ & $\begin{array}{l}\text { Reason } \\
\text { for oc- } \\
\text { clusion* }\end{array}$ \\
\hline 1 & 120 & $\leq 21$ days & Yes & 1 \\
\hline 2 & 15 & $\leq 21$ days & Yes & 1,2 \\
\hline 3 & 5 & $\leq 21$ days & Yes & 1 \\
\hline 4 & 0 & $\leq 21$ days & Yes & 3 \\
\hline 5 & 9 & $\leq 21$ days & Yes & 1 \\
\hline 6 & 9 & $\leq 21$ days & Yes & 1,2 \\
\hline 7 & 5 & $\leq 21$ days & Yes & 1,2 \\
\hline 8 & 42 & $\leq 21$ days & Yes & 1 \\
\hline 9 & 10 & $\leq 21$ days & Yes & 1,2 \\
\hline 10 & 41 & $\leq 21$ days & Yes & 2,4 \\
\hline 11 & 21 & $\leq 21$ days & Yes & 3 \\
\hline 12 & 56 & Unknown & No & 1 \\
\hline 13 & 83 & Unknown & No & 1 \\
\hline 14 & 120 & Unknown & No & 1 \\
\hline 15 & 40 & $>21$ days & No & 1,2 \\
\hline 16 & 35 & Unknown & No & 1 \\
\hline 17 & 30 & $>21$ days & No & 1 \\
\hline 18 & 93 & $>21$ days & No & 1 \\
\hline
\end{tabular}

*1 stent related failure e.g. stent fracture, kinking), 2 suboptimal stent position, 3 inadequate inflow, 4 failed anticoagulation

\section{Complications}

In total 6 patients experienced adverse events. During UACDT 2 patients had a hemoglobin decrease without a bleeding focus, which necessitated erythrocyte transfusion. One patient had minor bleeding at the catheter insertion site that was solved with manual compression. One patient experienced heparininduced thrombocytopenia. Heparin was switched for argatroban intravenous for the further duration of UACDT. One patient complained of blepharo edema and urticaria, which was recognized as an allergic reaction to iodine contrast fluid used for venographic control unrelated to thrombolytic therapy. One patient had fever with positive blood cultures during thrombolysis. In this patient thrombolysis was stopped and treatment with antibiotics was started. None of the patients experienced multiple complications. We did not encounter any 
clinically relevant pulmonary embolism or treatment related mortality Table 4 is an overview of all complications encountered during UACDT.

Table 4

\begin{tabular}{|lc|}
\hline Patients $(\mathrm{n}=18)$ & N (\%) \\
\hline Minor bleedinga $^{\mathbf{2}}$ & $1(5)$ \\
\hline Major bleedingb & $2(11)$ \\
\hline Pulmonary embolism & $0(0)$ \\
\hline Death & $0(0)$ \\
\hline Otherc & $3(17)$ \\
\hline
\end{tabular}

a Epistaxis, catheter insertion site.

b Drop in hemoglobin needing red blood cell transfusion.

c Allergic reaction, infection, heparin induced thrombocytopenia.

\section{Follow-up}

Regarding all successfully lysed patients $(n=11), 8$ remained patent, which translates into a primary patency of $73 \%$. Seven patients experienced complete relief of complaints and two patients had partial relief of complaints. One patients did not benefit from UACDT with regard to relief of complaints. In one case there was an immediate re-occlusion the day after successful lysis, due to stent related problems. Table 5 shows the follow-up data for the successfully lysed patients. 
Table 5

\begin{tabular}{|ll|}
\hline Follow-up $n=11$ \\
\hline $\begin{array}{l}\text { Follow-up length } \\
\text { Median in months (range) }\end{array}$ & 12 months $(0-41)$ \\
\hline $\begin{array}{l}\text { Patency at follow-up } \\
\text { Patent } \\
\text { Occluded }\end{array}$ & $73 \%(n=8)$ \\
\hline $\begin{array}{l}\text { Relief of complaints } \\
\text { Complete relief } \\
\text { Partial relief } \\
\text { No relief } \\
\text { unknown }\end{array}$ & $64 \%(n=3)$ \\
\hline
\end{tabular}

\section{Discussion}

With the growing number of effective thrombolytic treatments in acute iliofemoral DVT, we expect that an increasing number of patients will be additionally stented for residual stenotic lesions. Some reports show over $50 \%$ of patients are to be stented after thrombolysis. ${ }^{16}$ At the same time there are also more interventions performed in patients with post-thrombotic syndrome due to extensive chronic occlusions in the iliocaval tract. As both of these procedures gain in popularity, it can be expected that more venous stenting related complications will be encountered.

We identified reocclusion to be the most frequent and clinically important complication after venous stenting and by our knowledge we are the first to specifically focus on how to treat this important complication. Earlier studies also addressed stent occlusions to be an important complication after venous stenting. ${ }^{4,5,17}$

We report a technically successful recanalization of occluded stents in the venous system of $61 \%$ and a primary patency of $73 \%$ after a median follow-up of 12 months. In all cases it was possible to get a wire across the occluded segments. In all cases we intended to treat the stent occlusions with a suggested thrombus age of less than 21 days based on complaints or homogenicity on DUS. Thrombolysis within 21 days seems to result in more success than interventions at a later moment in our series. Although there is one report on successful interventions in the chronic DVT phase, we could not reproduce these findings. ${ }^{18}$ We believe it is important to accurately assess the time of onset of 
the thrombosis. We found that patient reported duration of complaints was not useful to determine thrombus age and therefore the success rate of the thombolysis was not predictable. Other techniques like MR-venography, DUS elastography, or intra vascular ultrasound might prove more useful in determining the thrombus age, better predict outcome and be used for patient selection. ${ }^{19}$

Regarding the specific usefulness of UACDT in regaining patency, we report a median lysis time to lyse $90 \%$ of the thrombus of 19 hours, which is fairly quick. However in some cases we continued the lysis treatment until the necessary additional procedures could be performed.

We encountered two rare complications; HIT and contrast allergy. Two major bleedings requiring transfusion were encountered. Other complications involved catheter insertion site bleeding, which were very minor, and therefore we may conclude that UACDT is a safe procedure.

Many stent related flow-complications result in re-occlusion of the venous tract. Occlusion of the stent tract nullifies the progress gained by the initial treatment. When encountered in an early stage, the thrombosed stent tract can be cleared by thrombolysis. ${ }^{4}$ Patency can be regained and most importantly the underlying cause of occlusion can be identified or should be identified during the procedure. UACDT is one of the available options for regaining patency. Other clot removal therapies can also be used for this purpose. ${ }^{20}$

In all cases of successful thrombolysis the underlying cause of the occlusion was identified. Also in the not successful thrombolysis group we identified stent related causes for occlusion. In total in 15 of all 18 cases we identified stent related failures like kinking, fracture and continuous compression of the stents. These stent related problems are potentially due to the stent characteristics. The stents used in this study are primarily designed for arterial use. With new, dedicated venous stents we expect to prevent future stent related occlusions. Ideally, dedicated venous stent with high flexibility, high radial force and large diameters are used in the venous system. ${ }^{21} \mathrm{~A}$ number of dedicated venous stents have become commercially available.

Most of the occlusions occurred within 6 months after the initial stenting. A rigid follow-up of patients is therefore paramount. The frequent follow up allowed for timely interventions with UACDT in case re-occlusion occurred. At the 6-month visit continuation of anticoagulation was evaluated based on stent configuration, patient risk factors and patient preference. Thrombocyte aggregation inhibitors were not routinely prescribed following cessation of Coumadin although they might be of influence. If at any point between follow-up visits patients experienced complaints, they were seen at the earliest convenience. 
After 6 months follow-up visits were planned at one year after stenting and afterwards yearly, depending on patient preference.

The most difficult problem we encountered is the patient with bad inflow due to extensive chronic venous occlusive disease with scarring in both major inflow vessels (i.e. femoral and deep femoral vein.). In these patients we noticed the impaired inflow due to extensive scarring of both the femoral and deep femoral vein. At the moment there are no reported successful treatments available for these veins. Endophlebectomy with or without AV-fistula has been described in literature as a treatment option in these cases. ${ }^{22-24}$ Stenting might be a possibility for these veins, however the current available material is not suitable for placement in the femoral or deep femoral tract. To reassure adequate inflow in the patient we currently rely on the creation of a temporary AVfistula, with or without an endophlebectomy of the common femoral vein to secure adequate inflow into the stented tract. ${ }^{25}$ Further research is needed to improve care in these cases.

One of the drawbacks of our study is the retrospective nature. The expected number of cases per year, per center is expected to be very low, which would makes it challenging to complete a prospective study. Optimal registration of patient data and pooling of results from retrospective studies could improve patient care considerably. We therefore would like to encourage other centers to report on their experience regarding stent thrombosis and its treatment.

\section{In conclusion}

Treatment with UACDT of occluded stent tracts is feasible and effective. Recanalization of the stent tract can be achieved in some cases with recent thrombosis less than 21 days. Additional interventions were frequently necessary after successful UACDT treatment. Most stent occlusions were caused by stent related problems. 


\section{References:}

1. Comerota AJ, Throm RC, Mathias SD, Haughton S, Mewissen M. Catheter-directed thrombolysis for iliofemoral deep venous thrombosis improves health-related quality of life. Journal of Vascular Surgery. 2000 Jul;32(1):130-7.

2. Mewissen MW, Seabrook GR, Meissner MH, Cynamon J, Labropoulos N, Haughton SH. Catheterdirected thrombolysis for lower extremity deep venous thrombosis: report of a national multicenter registry. Radiology. 1999 Apr;211(1):39-49.

3. Neglén P, Raju S. Balloon dilation and stenting of chronic iliac vein obstruction: technical aspects and early clinical outcome. J Endovasc Ther. 2000 Apr;7(2):79-91.

4. de Wolf MAF, Arnoldussen CW, Grommes J, Shu Gi H, Patricia Joan N, de Haan MW, et al. Minimally invasive treatment of chronic iliofemoral venous occlusive disease. Journal of Vascular Surgery. Society for Vascular Surgery; 2013 Apr 1;1(2):146-53.

5. Titus JM, Moise MA, Bena J, Lyden SP, Clair DG. Iliofemoral stenting for venous occlusive disease. Journal of Vascular Surgery. Elsevier Inc; 2011 Mar 1;53(3):706-12.

6. Mousa AY, Aburahma AF. May-Thurner Syndrome: Update and Review. Annals of Vascular Surgery. 2013 Jul 10.

7. Comerota AJ, Grewal N, Martinez JT, Chen JT, Disalle R, Andrews L, et al. Postthrombotic morbidity correlates with residual thrombus following catheter-directed thrombolysis for iliofemoral deep vein thrombosis. Journal of Vascular Surgery. 2012 Jan 24.

8. Raabe RD. Ultrasound-accelerated Thrombolysis in Arterial and Venous Peripheral Occlusions: Fibrinogen Level Effects. JVIR. Elsevier Inc; 2010 Aug 1;21(8):1165-72.

9. Engelhardt TC, Taylor AJ, Simprini LA, Kucher N. Catheter-directed ultrasound-accelerated thrombolysis for the treatment of acute pulmonary embolism. Thrombosis Research. 2011 Aug;128(2):149-54.

10. Strijkers RH, Grommes J, Arnoldussen CW, de Graaf R, Cate ten AJ, Wittens CH. Ultrasoundaccelerated catheter-directed thrombolysis in acute iliofemoral deep venous thrombosis. Journal of Vascular Surgery: Venous and Lymphatic Disorders. Elsevier; 2013.

11. Grommes J, Strijkers RHW, Greiner A, Mahnken AH, Wittens CHA. Safety and feasibility of ultrasound-accelerated catheter-directed thrombolysis in deep vein thrombosis. European Journal of Vascular \& Endovascular Surgery. 2011 Apr;41(4):526-32.

12. Strijkers RH, Arnoldussen CW, Wittens CH. Thrombectomy without lysis: the future? Phlebology. 2014 May 19;29(1 suppl):125-34.

13. Gornik HL, Sharma AM. Duplex ultrasound in the diagnosis of lower-extremity deep venous thrombosis. Circulation. 2014 Feb 25;129(8):917-21.

14. Vedantham S, Grassi CJ, Ferral H, Patel NH, Thorpe PE, Antonacci VP, et al. Reporting standards for endovascular treatment of lower extremity deep vein thrombosis. JVIR. 2009 Jul;20(7 Suppl):S391-408.

15. Broholm R, Panduro Jensen L, Baekgaard N. Catheter-directed thrombolysis in the treatment of iliofemoral venous thrombosis. A review. Int Angiol. 2010 Aug;29(4):292-302.

16. Neglén P, Hollis KC, Olivier J, Raju S. Stenting of the venous outflow in chronic venous disease: Long-term stent-related outcome, clinical, and hemodynamic result. Journal of Vascular Surgery. 2007 Nov;46(5):979-990.e1.

17. Dumantepe M, Tarhan IA, Ozler A. Treatment of Chronic Deep Vein Thrombosis Using Ultrasound Accelerated Catheter-directed Thrombolysis. European Journal of Vascular \& Endovascular Surgery. 2013 Jun 25.

18. Arnoldussen C, Strijkers R, Lambregts D, Lahaye M, de Graaf R, Wittens C. Feasibility of identifying deep vein thrombosis characteristics with contrast enhanced MR-Venography. Phlebology. 2014 May 19;29(1 suppl):119-24.

19. Karthikesalingam A, Young EL, Hinchliffe RJ, Loftus IM, Thompson MM, Holt PJE. A systematic review of percutaneous mechanical thrombectomy in the treatment of deep venous thrombosis. European Journal of Vascular \& Endovascular Surgery. 2011 Apr;41(4):554-65. 
20. de Graaf R, Arnoldussen C, Wittens CHA. Stenting for chronic venous obstructions a new era. Phlebology. 2013 Mar;28 Suppl 1:117-22.

21. Puggioni A, Kistner RL, Eklof B, Lurie F. Surgical disobliteration of postthrombotic deep veinsendophlebectomy-is feasible. Journal of Vascular Surgery. 2004 May;39(5):1048-52discussion52.

22. Raju S, Neglén P, Doolittle J, Meydrech EF. Axillary vein transfer in trabeculated postthrombotic veins. Journal of Vascular Surgery. 1999 Jun;29(6):1050-62-discussion1062-4.

23. Vogel D, Comerota AJ, Al-Jabouri M, Assi ZI. Common femoral endovenectomy with iliocaval endoluminal recanalization improves symptoms and quality of life in patients with postthrombotic iliofemoral obstruction. Journal of Vascular Surgery. Elsevier Inc; 2012 Jan 1;55(1):12935.

24. de Wolf MAF, Arnoldussen CWKP, Wittens CHA. Indications for endophlebectomy and/or arteriovenous fistula after stenting. Phlebology. 2013 Mar;28 Suppl 1:123-8. 


\section{CHAPTER 10}

Fibrinogen levels in patients undergoing thrombolytic therapy for acute iliofemoral deep venous thrombosis are extremely method dependent

Rob H.W. Strijkers

Gerhardus J. A. J. M. Kuiper

Rene van Oerle

Rick Wetzels

Arina J. ten Cate

Hugo ten Cate

Cees H.A. Wittens

Yvonne Henskens

In preparation 


\begin{abstract}
Introduction: Plasma fibrinogen levels are commonly used as a surrogate marker for predicting bleeding risk in patients undergoing thrombolysis to ensure patient safety. Difficulties in determining fibrinogen levels were encountered in patients undergoing ultrasound-accelerated thrombolysis for iliofemoral deep venous thrombosis.
\end{abstract}

Methods: From 2012 to 2014 fibrinogen levels were measured in patients undergoing ultrasound accelerated catheter directed thrombolysis for iliofemoral deep venous. Measurement errors were encountered in routine measurement of fibrinogen levels using the Clauss method with the Sysmex CS2100i (optical). Comparative measurements were performed using STA-R Evolution (mechanical) and Rotational ThromboElastoMetry on all samples, as well as absolute D-dimer levels.

Results: In total 87 samples from fifteen patients were obtained. A mean difference of $0.5 \mathrm{~g} / \mathrm{l}$ in plasma fibrinogen level for all samples $(95 \%$ confidence interval (95\% CI): 0.05-0.96) was found between the optical and mechanical method. Measurements with the optical method showed coagulation curve error in $41 \%$ of all samples, and in $2 \%$ with the mechanical method and no errors were observed with ROTEM FIBTEM. Samples without coagulation curve error $(n=49)$ had a median d-dimer level of $54 \mu \mathrm{g} / \mathrm{l}(\mathrm{IQR} 20.0-91.3)$ and $50 \mu \mathrm{g} / \mathrm{l}$ (27.5-118.5) for the group with coagulation curve error $(n=35)$. Ddimer levels did not differ significantly between the groups $(p=0.655)$. Based on laboratory testing 30 FFP's were given for the prevention of bleeding. Two minor bleeds occurred and no major bleeds.

Conclusion: Levels of plasma fibrinogen are method dependent in patients undergoing catheter directed thrombolysis for deep venous thrombosis. Levels of D-dimer do not seem to influence the results. Based on our results we assumed that the optical method underestimates fibrinogen levels 


\section{Introduction}

Fibrinogen measurements are commonly requested for monitoring hemostasis during thrombolytic therapy. ${ }^{1}$ Thrombolytic therapy is widely used in arterial thrombosis to actively dissolve the thrombus in cases of vessel occlusions e.g. peripheral arterial occlusion, massive pulmonary embolism, and ischemic cerebral vascular accidents. ${ }^{2-4}$ Lately, also iliofemoral deep venous thrombosis is sometimes treated with thrombolysis. Thrombolytics such as urokinase, t-PA, and rt-PA are tissue plasminogen activators, which activate endogenous plasminogen conversion into plasmin. The main function of plasmin is cleavage of fibrin strands thus destroying the fibrin matrix and dissolving the thrombus into fibrin degradation products. ${ }^{5}$ Urokinase has a direct catalytic activity against fibrinogen. ${ }^{6}$ In common practice fibrinogen levels are monitored to make sure the patient is still able to form clots and so prevent bleeding elsewhere in the body. The fibrinogen levels within an arbitrary value of 1.5 or $1.0 \mathrm{~g} / \mathrm{l}$ are commonly used as the minimum level of fibrinogen needed in order to prevent bleeding complications. ${ }^{7}$ The fibrinogen level is an important marker whereupon clinical decisions like continuation or cessation of thrombolytic therapy are made. Lower fibrinogen levels were associated with a higher bleeding risk in cardiac patients. ${ }^{8}$ Subsequent addition of fibrinogen could possibly prevent bleeding in patients. However addition of fibrinogen with either fresh frozen plasma (FFP) or fibrinogen monotherapy is associated with increased morbidity, mortality and costs. ${ }^{9-12}$ Accurate fibrinogen measurement is of the utmost importance to make sound clinical decisions and to secure the patients' safety regarding major bleeding complications during thrombolytic therapy. Plasma fibrinogen levels can be determined by a number of different assays, both optical and mechanical. With regard to routine coagulation testing these methods are deemed equal. ${ }^{13}$ The Clauss method is the most often used mechanical method. This is a functional assay based on the time taken for fibrin clot formation upon addition of thrombin.

ROtational ThromboElastoMetry (ROTEM) has been evaluated in surgical patients and patients with major blood loss. ROTEM, and especially FIBTEM, is extensively used during cardiac and hepatic surgery, when massive bleeding occurs, and in trauma patients. ${ }^{14}$ ROTEM FIBTEM provides a quick and easy alternative to the standard coagulation tests. FIBTEM has been positively correlated with fibrinogen levels in various patient groups, ${ }^{15-17}$ and reference values have been determined.18,19 However FIBTEM values during thrombolysis is not yet considered for routine measurement of fibrinogen levels.

Difficulties in determining fibrinogen levels were encountered in patients undergoing catheter directed thrombolysis for iliofemoral deep venous thrombosis using the Clauss method. This article describes the results for the com- 
parison of two different clot detection methods for the determination of plasma fibrinogen levels (optical and mechanical) and for the functional fibrinogen method (FIBTEM) by ROTEM in patients undergoing thrombolysis treatment for acute iliofemoral DVT.

\section{Material and Methods}

\section{Patients:}

From August 2012 till June 2014 consecutive patients who were included in the CAVA study (METC 11-4-097) and who were randomized to undergo catheter directed thrombolysis for acute iliofemoral deep venous thrombosis were eligible for inclusion in the comparative laboratory study. Before inclusion in the CAVA study, all patients were screened for elevated bleeding risk using the criteria shown in table 1 . Fibrinogen levels were measured during the procedure; back up plasma samples were stored for future comparison if required. Data acquisition and analyses was performed anonymously in accordance with the Dutch Law for approving medical research.

\section{Table 1}

\section{Exclusion criteria for catheter directed thrombolysis}

History of Gastro-intestinal bleeding within 6 months

History of Cerebral Vascular Accident (CVA)/central nervous system disease

Severe hypertension ( $>180 / 100 \mathrm{mmHg}$ )

Active malignancy

Surgery within 2 weeks

Previous thrombosis of the affected limb (secondary thrombosis)

Pregnancy

Alanine transaminase(ALAT) $>3$ times normal range

Glomular Filtration Rate (eGFR) $<30 \mathrm{ml} / \mathrm{min}$

\section{Treatment:}

All patients were treated with the EKOS endowave ultrasound accelerated catheter directed thrombolysis (UACDT) (BTG, USA) and as thrombolytic agent urokinase (Medacinase, Lamepro) was used. The EKOS endowave makes use of ultrasound elements to make fibrin strands more susceptible for thrombolysis and to distributes the thrombolytic drug more evenly throughout the clot. The 
ultrasounds elements are cooled with room temperature saline solution given with a minimum of $35 \mathrm{ml} /$ hour and a maximum of $100 \mathrm{ml} /$ hour. The outer lumen consists of a multi side-hole catheter, which infuses the thrombolytic drug into the clot.

\section{Blood drawing and plasma preparation:}

Blood was collected in $4.5 \mathrm{ml}, 3.2 \%$ citrate tubes for analysis of fibrinogen and ROTEM tests and in EDTA tubes for hemocytometry (BD Vacutainer, Becton Dickinson, NJ, USA). Laboratory measurements were performed for routine patient care and comparative study purposes. Citrated plasma was prepared by centrifugation for 5 minutes at 3790 revolutions per minute. For direct analysis plasma was prepared at $2000 \mathrm{~g}$ for 10 minutes. Preparation of the frozen plasma samples was carried out at $2500 \mathrm{~g}$ for 5 minutes and after pipetting residual contents again at $10,000 \mathrm{~g}$ for 10 minutes.

\section{Laboratory measurements}

Pre-treatment hemoglobin ( $\mathrm{Hb}$ ) levels, international normalized ratio (INR), prothrombin time (PT), D-dimer and plasma fibrinogen levels were assessed. During thrombolysis blood was drawn routinely, every six hours and Hb levels, activated partial thromboplastin time (aPTT), PT, INR, plasma fibrinogen, platelet count, and D-dimer levels were assessed.

Hemostasis parameters including fibrinogen measurements were performed on a Sysmex Cs-2100-I. Fibrinogen levels were determined using the Clauss coagulation method (Dade Thrombin Reagent, Siemens Healthcare Diagnostics Products, Marburg, Germany). ${ }^{20}$ Also routine measurements with ROTEM were performed at the same time in whole blood and one additional citrated back up plasma sample was stored at -80 degrees Celsius for later comparative analysis on CS2100-I and STA-R evolution. ROTEM tests and conventional laboratory tests were performed simultaneously in the central laboratory by trained laboratory staff. Both conventional laboratory tests and ROTEM tests were part of the standard diagnostic procedure during thrombolysis.

The Clauss method, is a functional assay based upon the time for fibrin clot formation. Clot detection in automated hemostasis analyzers can either be optical (Sysmex CS2100) or mechanical (STA-R Evolution, Stago). Another approach to fibrinogen measurement is the functional fibrinogen test in whole blood using the fibrin specific ROtational ThromboElastoMetry (ROTEM). ROTEM analysis for coagulation monitoring in bleeding patients is increasingly used because of ROTEM analysis' speed and as a predictor of global hemostasis function. ${ }^{14}$ The method used is based on changes in viscoelasticity of activated whole blood due to cloth formation. A rotating pin emerged in the activated 
whole blood is restricted in movement by the formation of the clot. This is plotted in a graph and given by parameters like the time till the first clot (CT or clotting time), maximum clot strength (MCF or Maximum Clot Formation), and lysis parameters (e.g. LY30 is the lysis index after 30 minutes). ${ }^{14}$ Thromboelastometric measurements were performed with the ROTEM device (ROTEM $®$, TEM International GmbH, Munich, Germany) in citrated whole blood. Measurements were performed at $37^{\circ} \mathrm{C}$ immediately after blood draw according to the instructions of the manufacturer. All pipetting steps and mixing of reagents (TEM Innovations GmbH, Munich, Germany) were performed with the automated electronic pipette. FIBTEM MCF values were analyzed. FIBTEM tests coagulation is extrinsically activated by tissue factor. In the FIBTEM tests a platelet inhibitor was added (cytochalasin D) to correct for the contribution of platelets to the clot strength. Normal population reference values for Fibtem MCF in reported literature and according to the manufacturer is $9-25 \mathrm{~mm}$, which should correlate to the normal CLauss determined fibrinogen levels 1.54.5g/l. ${ }^{18}$ Correlation between the Clauss method and fibtem MCF has been described, where $<10 \mathrm{~mm}$ fibtem MCF correlated well with $1.5 \mathrm{~g} / \mathrm{l}$ fibrinogen. ${ }^{21}$ Because $1.0 \mathrm{~g} / \mathrm{l}$ is used as lower limit during thrombolysis, the lower limit for Fibtem MCF was extrapolated and set at $6 \mathrm{~mm}$.

At a later point in time fibrinogen levels in the frozen samples were measured using STA-R Evolution (STA® Liquid Fib reagens, Diagnostica Stago) and fibrinogen measurements using CS2100-I fibrinogen analysis was repeated in the same thawed plasma sample..

D-dimer was analyzed with BCS XP using INNOVANCE D-Dimer reagens (Siemens Healthcare Diagnostics Products, Marburg, Germany). Absolute D-dimer levels were assessed using a 100 or 200 fold dilution. Analysis of D-dimer levels was performed to evaluate potential disturbances in the optical fibrinogen measurements. Routine hematology measurements of whole blood samples were carried out on an automated analyser XE-2100 (Sysmex Corporation, Kobe, Japan).

\section{Treatment drugs and transfusion}

For the thrombolysis procedure Urokinase was started using a bolus of 250.000 IE and continued with 100.000 IE/hour diluted in $20 \mathrm{cc} 0.9 \%$ saline solution. If plasma fibrinogen level dropped below $1.0 \mathrm{~g} / \mathrm{l}$, dosage of urokinase was halved to 50.000IE/hour and 2 FFP's were given.

The fibrinogen level was checked, 3 hours after infusion of FFP's. If the plasma fibrinogen level persisted to be below $1.0 \mathrm{~g} / \mathrm{l}$ than thrombolysis was paused and another infusion of FFP's was given. After 3 hours, again plasma fibrinogen level was measured and when fibrinogen was above $1.0 \mathrm{~g} / \mathrm{l}$, thrombolytic ther- 
apy was continued if perceived necessary. During thrombolysis unfractionated heparin (Leo Pharma, 1000EI/h in 2cc saline solution, starting bolus 5000EI) was administered to prevent new clots from forming. Heparin was controlled by measurement of aPTT levels, that were aimed between 1.2-1.7 times the reference value, which translates to 40-60 seconds for aPTT. Hemoglobin levels should not drop more than 1 point between measurements. Thrombolysis was continued until $>90 \%$ clot resolution and should not exceed the maximum of 96 hours of thrombolysis.

\section{Statistical analysis}

Continuous data are shown as median and interquartile range (IQR) when skewed distribution is apparent and mean plus standard deviation (SD) when data follow normal distribution. Categorical data are shown in absolute numbers and percentages, unless otherwise stated. Pearson's correlation coefficient ( ) was calculated to determine the correlation between ROTEM variables and conventional laboratory tests. Non-parametric Mann-Whitney U test was performed to determine differences between fibrinogen levels and D-dimer levels. Differences between the CS2100i and STA-R are shown in a Bland-Altman plot. All statistical analyses were performed using commercially available computer software (SPSS, Version 22.0, SPSS, Inc., Chicago, IL).

\section{Results}

\section{Fibrinogen}

There is a notable difference in reported levels of fibrinogen with STA showing higher levels of fibrinogen levels in all samples compared to CS2100-I. The mean difference is $0.5 \mathrm{~g} / \mathrm{l}$ for all samples (95\% confidence interval (95\% CI): 0.05-0.96) with higher values in the STA samples. Figure 1 shows the BlandAltman plot of the difference between the reported fibrinogen levels by CS2100-I and STA-R. 


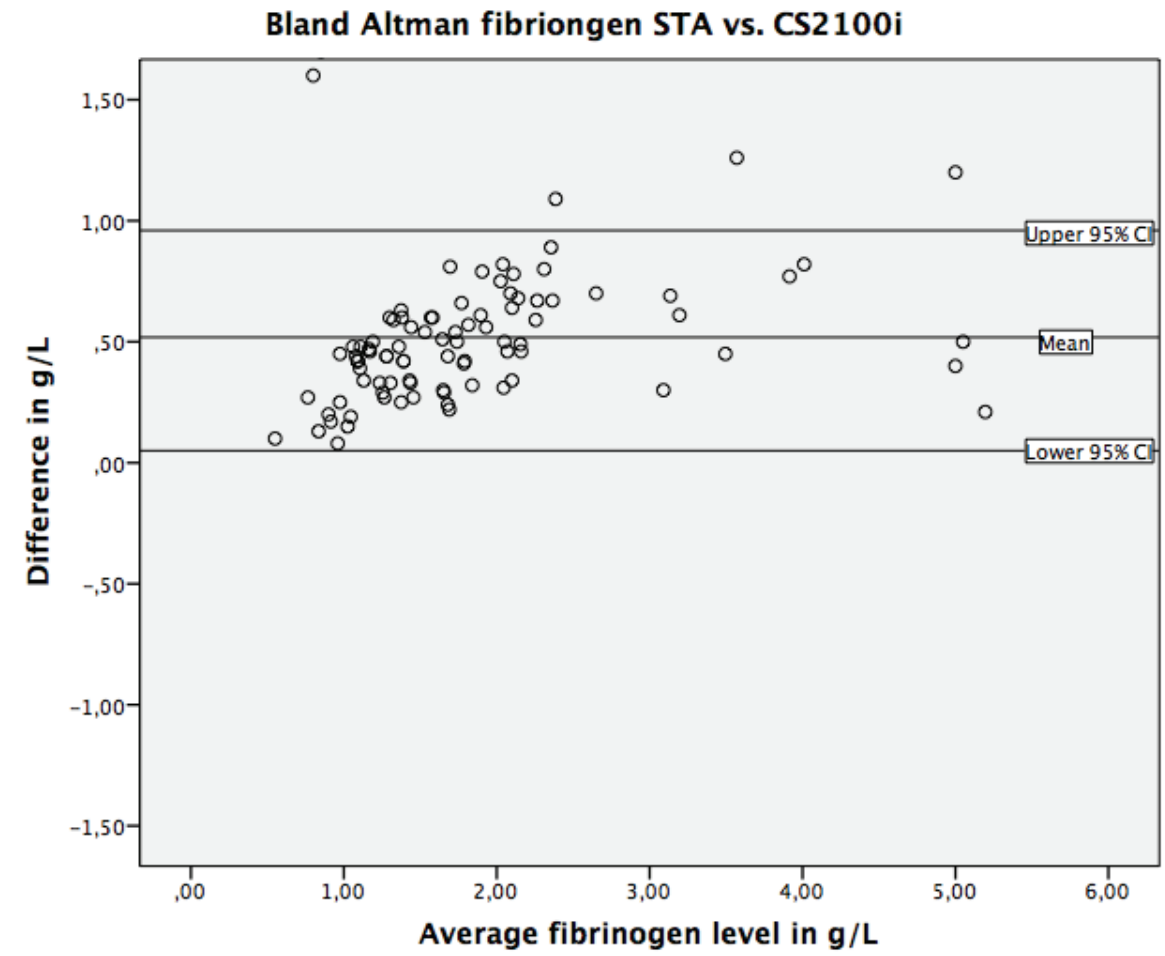

Figure 1: This Bland Altman plot shows the difference in fibrinogen levels between the STA and CS2100i. The plot shows consistent higher values with STA compared to the corresponding CS2100i samples.

ROTEM results were obtained from 15 patients who underwent UACDT for DVT. In total 87 ROTEM FIBTEM results were analyzed in the acute phase. In all cases routinely measured CS2100-I fibrinogen results and additional stored samples were available. The number of analyses per patient ranged from 1 to 17. Baseline characteristics and baseline fibrinogen level per patient are shown in Table 2. Table 3 shows the median aPTT levels; median hemoglobin level, platelet counts and PT per patient during thrombolysis. 
Table 2

\begin{tabular}{|llll|}
\hline Patient characteristics & Age in years & Gender & $\begin{array}{l}\text { Baseline fibrinogen } \\
\text { level in g/l on } \\
\text { CS2100i (reference } \\
\text { value: 1.7-4.0) }\end{array}$ \\
\hline Patient 1 & 47 & Male & 4.8 \\
\hline Patient 2 & 16 & Female & N/A \\
\hline Patient 3 & 19 & Female & 8.0 \\
\hline Patient 4 & 52 & Male & 6.9 \\
\hline Patient 5 & 76 & Female & 4.4 \\
\hline Patient 6 & 65 & Male & 6.0 \\
\hline Patient 7 & 18 & Female & 5.4 \\
\hline Patient 8 & 53 & Female & 7.1 \\
\hline Patient 9 & 69 & Male & 2.9 \\
\hline Patient 10 & 18 & Male & 3.0 \\
\hline Patient 11 & 16 & Female & 4.8 \\
\hline Patient 12 & 27 & Male & 3.3 \\
\hline Patient 13 & 61 & Male & 4.4 \\
\hline Patient 14 & 51 & Male & 5.4 \\
\hline Patient 15 & 42 & Female & 3.2 \\
\hline N/A nt avale & & \\
\hline
\end{tabular}

$\mathrm{N} / \mathrm{A}=$ not available. 
Table 3 displays the median hemostatic values during thrombolysis per patient.

\begin{tabular}{|c|c|c|c|c|c|c|}
\hline & $\begin{array}{l}\text { Available } \\
\text { samples }\end{array}$ & $\begin{array}{l}\text { Hemoglobin } \\
\text { median value }\end{array}$ & $\begin{array}{l}\text { Aptt in } \\
\text { seconds } \\
\text { median } \\
\text { value }\end{array}$ & $\begin{array}{l}\text { Thrombocytes } \\
\text { median value }\end{array}$ & $\begin{array}{l}\text { Pt in } \\
\text { seconds } \\
\text { Median }\end{array}$ & $\begin{array}{l}\text { Percentage of } \\
\text { coag curve } \\
\text { error with } \\
\text { CS2100i }\end{array}$ \\
\hline $\begin{array}{l}\text { Reference } \\
\text { values }\end{array}$ & $\begin{array}{l}\text { Total } \\
\text { samples=87 }\end{array}$ & $\begin{array}{l}8.2- \\
11.0 \mathrm{mmol} / \mathrm{L}\end{array}$ & $23-32 \mathrm{sec}$ & $\begin{array}{l}130- \\
350^{*} 10^{\wedge} 9 \backslash \mathrm{L}\end{array}$ & $\begin{array}{l}9.9- \\
11.5 \mathrm{sec}\end{array}$ & \\
\hline Patient 1 & 15 & 5.4 & $44 \mathrm{sec}$ & 255 & $19.4 \mathrm{sec}$ & $33 \%(n=5)$ \\
\hline Patient 2 & 2 & 7.4 & $80 \mathrm{sec}$ & 295 & $20.6 \mathrm{sec}$ & $100 \%(\mathrm{n}=2)$ \\
\hline Patient 3 & 10 & 5.9 & $45 \mathrm{sec}$ & 225 & $12.6 \mathrm{sec}$ & $30 \%(n=3)$ \\
\hline Patient 4 & 6 & 6.9 & $45 \mathrm{sec}$ & 237 & $12.5 \mathrm{sec}$ & $0 \%$ \\
\hline Patient 5 & 6 & 7.0 & $38 \mathrm{sec}$ & 300 & $13.4 \mathrm{sec}$ & $0 \%$ \\
\hline Patient 6 & 2 & 8.2 & $44 \mathrm{sec}$ & 154 & $16.5 \mathrm{sec}$ & $0 \%$ \\
\hline Patient 7 & 17 & 5.3 & $46 \mathrm{sec}$ & 443 & $12.5 \mathrm{sec}$ & $71 \%(n=12)$ \\
\hline Patient 8 & 2 & 7.1 & $41 \mathrm{sec}$ & 228 & - & $0 \%$ \\
\hline Patient 9 & 1 & 9.6 & $57 \mathrm{sec}$ & 251 & - & $100 \%$ \\
\hline Patient 10 & 1 & 9.1 & $50 \mathrm{sec}$ & 254 & - & $0 \%$ \\
\hline Patient 11 & 6 & 6.3 & $43 \mathrm{sec}$ & 206 & $13.6 \mathrm{sec}$ & $67 \%(n=4)$ \\
\hline Patient 12 & 4 & 8.3 & $55 \mathrm{sec}$ & 111 & $12.7 \mathrm{sec}$ & $50 \%(n=2)$ \\
\hline Patient 13 & 7 & 9.3 & $129 \mathrm{sec}$ & 171 & $19.8 \mathrm{sec}$ & $29 \%(\mathrm{n}=2)$ \\
\hline Patient 14 & 4 & 6.7 & $58 \mathrm{sec}$ & 78 & $42.1 \mathrm{sec}$ & $100 \%$ \\
\hline Patient 15 & 4 & 7.8 & $39 \mathrm{sec}$ & 319 & $11.7 \mathrm{sec}$ & $25 \%(n=1)$ \\
\hline
\end{tabular}

The CS2100-I reported coagulation curve error in numerous samples. The analyzer in these cases displayed fibrinogen results however the reported level was not reliable according to the manufacturer. This non-reported number was compared to STA-go and FIBTEM MCF outcomes. In 41\% of the samples $(n=36)$, the CS2100-I analyzer with optical clot detection reported a coagulation curve error. By using STA-R, with mechanical clot detection, 98\% of samples showed detectable levels of fibrin $(n=85)$.

Figure 2 shows the correlation between CS2100-I and FIBTEM MCF. The value for intervention for CS2100-I and STA-R are set to $1.0 \mathrm{~g} / \mathrm{l}$ and $6 \mathrm{~mm}$ for ROTEM FIBTEM MCF. Measured values were lower than $1.0 \mathrm{~g} / \mathrm{l}$ in twenty samples with CS2100-I, in two samples with the STA-R and in six samples with the FIBTEM MCF $(<6 \mathrm{~mm})$. 
Figure 2

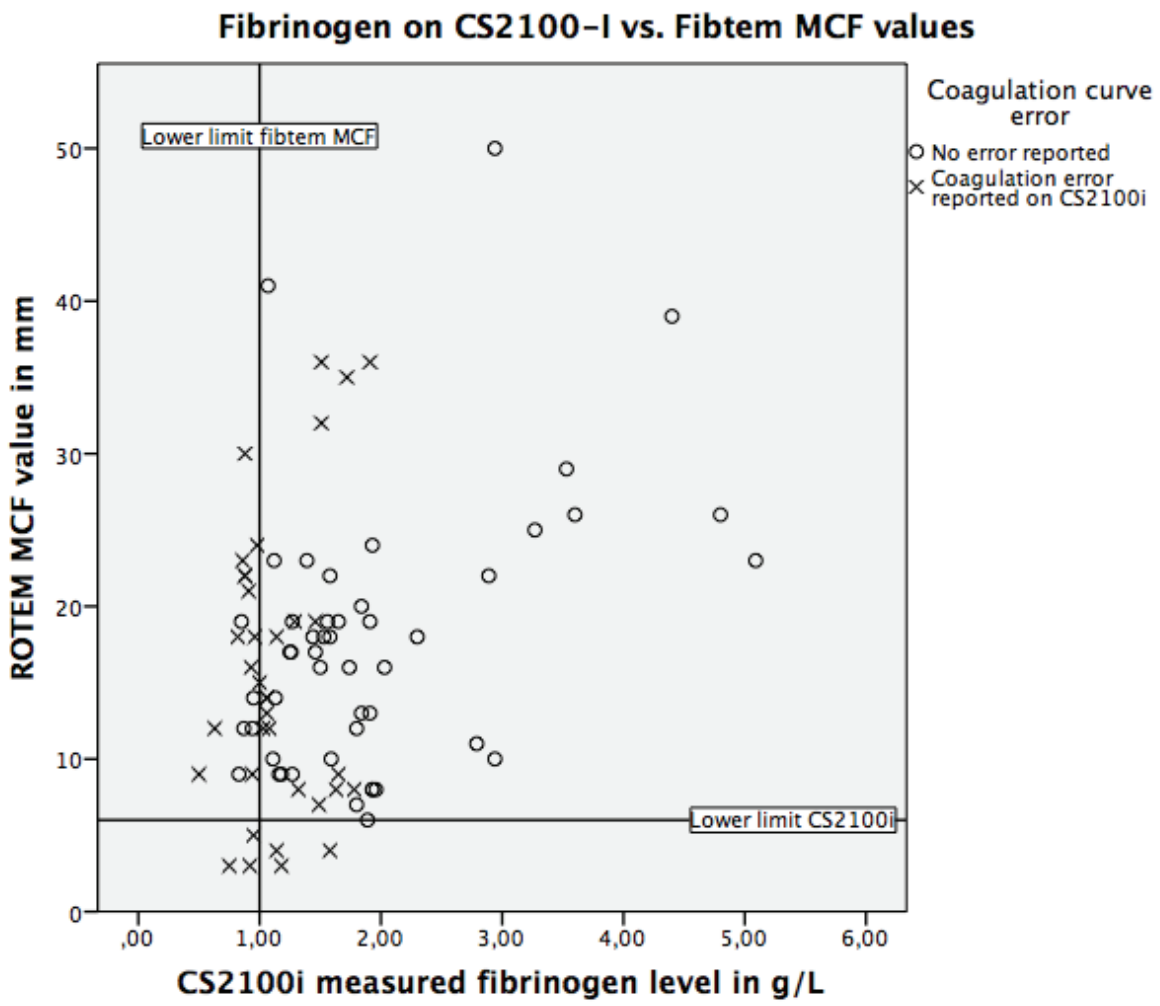

D-Dimer

Absolute D-dimers were assessed in $95 \%$ of samples after dilution $(n=84)$.

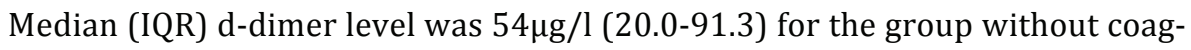
ulation curve error $(n=49)$ and $50 \mu \mathrm{g} / \mathrm{l}(27.5-118.5)$ for the group with coagulation curve error $(n=35)$. D-dimer levels did not differ significantly between the groups $(\mathrm{p}=0.655)$.

\section{Coagulation curve error}

Error reports were encountered at low fibrinogen levels. Error reports were encountered with the optical method in $41 \%$ (36/87). The STA method produced fibrinogen levels in $98 \%$ of samples (85/87). In all cases FIBTEM MCF results were available. In 36 samples with coagulation curve error on CS2100-I, median (IQR) reported fibrinogen levels were $1.1 \mathrm{~g} / \mathrm{l}(0.9-1.5)$ compared to STA-go samples with a median 1.6g/l (1.3-2.0) $(n=35)$. The group without coagulation curve error showed a median of 1.7g/l (1.3-2.0) $(n=49)$ with CS2100- 
I and 2.2g/l (1.6-2.7) (n=51) with STA-go. There was a significant difference between de CS2100-I group with and without coagulation curve error in terms of fibrinogen level $(\mathrm{p}<0.001)$ and also between the CS2100 and STA group with coagulation curve error $(\mathrm{p}<0.001)$.

\section{Safety and complications:}

Based on the hospital protocol, patients are transfused with FFP when fibrinogen levels drop below $1.0 \mathrm{~g} / \mathrm{l}$ measured by the routine method, CS2100-I. In total 30 units of FFP were administered in $53 \%$ of patients $(n=8)$. Three patients received up to 6 FFP's to correct fibrinogen levels under $1.0 \mathrm{~g} / \mathrm{l}$. Based on comparative STA-R results, retrospectively determined, only one patient should have received FFP's. Four patients would have received FFP if FIBTEM MCF measurements using $6 \mathrm{~mm}$ as a cut off value had been used to manage patients. Two minor bleedings were reported during thrombolysis, one at the catheter insertion site and one patient experienced clinically relevant epistaxis. No major bleedings were reported.

\section{Discussion}

Fibrinogen levels are used to monitor the patient's risk for bleeding during active thrombolysis. To ensure the patients safety with regards to bleeding risk, fibrinogen levels are monitored frequently during the thrombolysis procedure. The Hospital protocol in use defines $1.0 \mathrm{~g} / \mathrm{l}$ as the lower limit. Low levels of plasma fibrinogen were encountered frequently with the use of CS2100-I while STA and ROTEM results were within normal limits. Moreover, the correlating FIBTEM MCF value analyses showed that in 81 out of $87(93 \%)$ of all samples a functional clot could be formed, indicating that fibrinogen levels were sufficiently high. Therefore post hoc comparison testing was performed to assess the validity of the results determined by the Clauss method.

It has been suggested by some authors that the optical method probably gives the best estimate of functional fibrinogen. ${ }^{22}$ It is also suggested that determination of fibrinogen levels in optical detection methods may be disturbed by high D-dimer levels.. This hypothesis was however not corroborated by the presented data, no statistically significant difference in D-dimer levels was found between the samples with and without errors in this study. The abundance of fibrinogen degradation products due to thrombolysis does not seem to influence test results, other factors that interfere with accurate measurement should therefore be evaluated. Because currently used fibrinogen levels are arbitrary, alternative ROTEM values may be studied. 
FIBTEM MCF is an alternative to the optical and mechanical methods for determining plasma fibrinogen levels. Numerous publications describe the use of ROTEM in cardiothoracic surgery and in patients with high bleeding tendencies. ${ }^{8,23}$ ROTEM can provide an adequate status of the coagulation capacity of the patient and the clot formation potential. The method could therefore potentially be used for monitoring patients undergoing thrombolysis for DVT. Previous reports showed good correlation between FIBTEM MCF and alternative fibrinogen detection methods in healthy subjects. ${ }^{17}$ Also the correlation between FIBTEM MCF and CS2100-I in patients undergoing thrombolysis for DVT was good. During thrombolysis minor bleeding was observed in two patients and low fibrinogen in 8 patients, for which they received in total $30 \mathrm{FFP}$ 's. The low incidence of bleeding and the frequently reported low fibrinogen suggest an underestimation of fibrinogen levels with optical fibrinogen assessment.

Some concerns could be raised on the fact that samples for the current study were frozen and stored for later evaluation, this may be perceived as a weakness of our study as this could have influenced the results. However, additional duplicate testing showed no significant differences between the stored samples and fresh blood samples (results are not shown). STA results were obtained only from frozen samples. Accuracy of the STA analysis was assumed based on this control experiment.

From our comparative analysis we conclude that accurate measurement of fibrinogen levels in patients undergoing thrombolysis in acute DVT is challenging. Currently available methods of determination by CS2100-I or STA may not adequately reflect actual fibrinogen levels. Based on our experience and validation of ROTEM an underestimation of fibrinogen levels with CS2100-I in patients undergoing thrombolysis for acute iliofemoral DVT is assumed. 


\section{References:}

1. Castaneda F, Li R, Young K, Swischuk JL, Smouse B, Brady T. Catheter-directed thrombolysis in deep venous thrombosis with use of reteplase: immediate results and complications from a pilot study. JVIR. 2002 Jun;13(6):577-80.

2. Engelhardt TC, Taylor AJ, Simprini LA, Kucher N. Catheter-directed ultrasound-accelerated thrombolysis for the treatment of acute pulmonary embolism. Thrombosis Research. 2011 Aug;128(2):149-54.

3. Quintana D, Salsamendi J, Fourzali R, Narayanan G. Ultrasound-Assisted Thrombolysis in Submassive and Massive Pulmonary Embolism: Assessment of Lung Obstruction Before and After Catheter-Directed Therapy. Cardiovasc Intervent Radiol. 2013 Jul 17.

4. Baldwin ZK, Comerota AJ, Schwartz LB. Catheter-directed thrombolysis for deep venous thrombosis. Vascular and Endovascular Surgery. 2004;38(1):1-9.

5. Alkjaersig N, Fletcher AP, Sherry S. The mechanism of clot dissolution by plasmin. J Clin Invest. 1959 Jul;38(7):1086-95.

6. Weitz JI, Leslie B. Urokinase has direct catalytic activity against fibrinogen and renders it less clottable by thrombin. J Clin Invest. 1990 Jul;86(1):203-12.

7. Ouriel K, Kandarpa K. Safety of thrombolytic therapy with urokinase or recombinant tissue plasminogen activator for peripheral arterial occlusion: a comprehensive compilation of published work. J Endovasc Ther. 2004 Aug;11(4):436-46.

8. Gielen C, Dekkers O, Stijnen T, Schoones J, Brand A, Klautz R, et al. The effects of pre- and postoperative fibrinogen levels on blood loss after cardiac surgery: a systematic review and metaanalysis. Interactive CardioVascular and Thoracic Surgery. 2014 Feb 15;18(3):292-8.

9. Dara SI, Rana R, Afessa B, Moore SB, Gajic O. Fresh frozen plasma transfusion in critically ill medical patients with coagulopathy. Crit Care Med. 2005 Nov;33(11):2667-71.

10. Gajic 0, Dzik WH, Toy P. Fresh frozen plasma and platelet transfusion for nonbleeding patients in the intensive care unit: benefit or harm? Crit Care Med. 2006 May;34(5 Suppl):S170-3.

11. Khan H, Belsher J, Yilmaz M, Afessa B, Winters JL, Moore SB, et al. Fresh-frozen plasma and platelet transfusions are associated with development of acute lung injury in critically ill medical patients. Chest. 2007 May;131(5):1308-14.

12. Sarani B, Dunkman WJ, Dean L, Sonnad S, Rohrbach JI, Gracias VH. Transfusion of fresh frozen plasma in critically ill surgical patients is associated with an increased risk of infection. Crit Care Med. 2008 Apr;36(4):1114-8.

13. Tekkesin N, Kılınc C. Optical and Mechanical Clot Detection Methodologies: A Comparison Study for Routine Coagulation Testing. J Clin Lab Anal. 2012 May 24;26(3):125-9.

14. Ganter MT, Hofer CK. Coagulation monitoring: current techniques and clinical use of viscoelastic point-of-care coagulation devices. Anesthesia \& Analgesia. 2008 May;106(5):1366-75.

15. Rugeri L, Levrat A, David JS, Delecroix E, Floccard B, Gros A, et al. Diagnosis of early coagulation abnormalities in trauma patients by rotation thrombelastography. Journal of Thrombosis and Haemostasis. 2007 Feb;5(2):289-95.

16. Schöchl H, Nienaber U, Hofer G, Voelckel W, Jambor C, Scharbert G, et al. Goal-directed coagulation management of major trauma patients using thromboelastometry (ROTEM)-guided administration of fibrinogen concentrate and prothrombin complex concentrate. Crit Care. 2010;14(2):R55.

17. Engberink RHGO, Kuiper GJAJM, Wetzels RJH, Nelemans PJ, Lance MD, Beckers EAM, et al. ORIGINAL ARTICLES. Journal of Cardiothoracic and Vascular Anesthesia. Elsevier; 2014 Apr $1 ; 28(2): 210-6$.

18. Lang T, Bauters A, Braun SL, Pötzsch B, Pape von K-W, Kolde H-J, et al. Multi-centre investigation on reference ranges for ROTEM thromboelastometry. Blood Coagul Fibrinolysis. 2005 Jun;16(4):301-10. 
19. Whiting D, DiNardo JA. TEG and ROTEM: technology and clinical applications. Am J Hematol. 2014 Feb;89(2):228-32.

20. CLAUSS A. [Rapid physiological coagulation method in determination of fibrinogen]. Acta Haematol. 1957 Apr;17(4):237-46.

21. Meyer MAS, Ostrowski SR, Sørensen AM, Meyer ASP, Holcomb JB, Wade CE, et al. Fibrinogen in trauma, an evaluation of thrombelastography and rotational thromboelastometry fibrinogen assays. J Surg Res. 2014 Nov 20.

22. Weerkamp F, de Maat M. Interference of Fibrinogen Measurement by Fibrin Degradation Products During Thrombolysis. Int. Jnl. Lab. Hem. 2014. pp. 1-136.

23. Solomon C, Cadamuro J, Ziegler B, Schöchl H, Varvenne M, Sørensen B, et al. A comparison of fibrinogen measurement methods with fibrin clot elasticity assessed by thromboelastometry, before and after administration of fibrinogen concentrate in cardiac surgery patients. Transfusion. 2011 Feb 25;51(8):1695-706.

24. Skeik N, Gits CC, Ehrenwald E, Cragg AH. Fibrinogen Level as a Surrogate for the Outcome of Thrombolytic Therapy Using Tissue Plasminogen Activator for Acute Lower Extremity Intravascular Thrombosis. Vascular and Endovascular Surgery. 2013 Sep 19;47(7):519-23. 


\section{CHAPTER 11}

\section{Thrombectomy without lysis: the fu-}

ture?

Rob H.W. Strijkers

Carsten W.K.P. Arnoldussen

Cees H.A. Wittens

Phlebology 2014, Vol. 29(1S) 125-134 


\begin{abstract}
The results of the CaVent-study and the expected results of the ATTRACT and CAVA trials will form the base of evidence to support that rapid cloth removal is beneficial for patients with iliofemoral DVT. Although beneficial, there are still significant risks associated with this therapy. Therefore alternative methods for rapid cloth removal without lysis are a potential valid alternative. In this article we describe the techniques currently available for thrombolysis and discuss the potential improvements to be made to clot removal techniques in the future.
\end{abstract}


Introduction:

Thrombolytic therapy for Deep Venous Thrombosis (DVT) is becoming more acceptable in common practices. The randomized controlled CaVenT-study showed favorable results in patients treated with catheter directed thrombolysis (CDT) compared to patients who only received standard anticoagulation therapy with regards to Post Thrombotic Syndrome (PTS).(1) Currently 2 randomized controlled trials are conducted and soon the results of these trials are expected. $(2,3)$ If these trials confirm the favorable results with regards to prevention of PTS, CDT for iliofemoral DVT will probably become standard therapy in patients with iliofemoral DVT. While catheter directed thrombolytic therapy might prove to be favorable for patients with iliofemoral DVT it is not the ideal treatment.(4) Hence alternatives to CDT for iliofemoral DVT are being developed and investigated. In this article we describe the techniques currently available for thrombolysis and discusses the potential improvements of clot removal techniques in the future.

\section{Standard anticoagulation treatment}

Standard anticoagulation therapy prevents clot propagation and protects from potentially lethal pulmonary embolism.(5) Because the natural recanalization process in the popliteal-femoral tract is good, in up to $90 \%$ of cases standard care seems sufficient. However in the iliac tract the natural recanalization is much poorer with reported recanalization rates of only $15-30 \%$. The remaining clot transforms into fibrous tissue between 3-6 weeks till 3 months and up to one year collaterals are formed.(6) The fibrous tissue formed, as a result of incomplete recanalization, blocks venous outflow and is viewed as the major cause of the post thrombotic syndrome. (7)

\section{Aim of thrombolytic therapy:}

Thrombolytic therapy is used to quickly resolve the thrombus in an acute phase (within three weeks). With successful acute removal of the thrombus, full recanalization is gained and the formation of fibrous strands within the vein is prevented, therefore hopefully preventing the development of PTS.(8)

\section{Standard catheter directed thrombolysis (CDT):}

Standard catheter directed thrombolysis infuses the thrombolytics through a multiside hole catheter.(9) Urokinase, T-PA or rT-PA is used as thrombolytic agent in this treatment. The randomised controlled CaVenT-study showed safety and feasibility of this treatment with a mean treatment time of 2.4 days using standard CDT, with acceptable bleeding risk. Regarding PTS an absolute risk reduction of $14.4 \%$ was shown in patients treated with CDT in addition to standard anticoagulation treatment. 


\section{Pharmaco-mechanical thrombolysis (PMT)}

Because of the long treatment time of CDT, new treatment modalities have become available and combine a mechanical component with the chemical thrombolytic agent. The addition of a mechanical component is supposed to speed up the process of thrombolysis by disrupting the structure of the thrombus, thus shortening treatment time and possibly reducing the risk of bleeding due to the use of less thrombolytics. Three PMT devices are available for use: The EKOS endowave system (BTG, USA), the trellis-8 (Covidien) and the Angiojet powerpulse system (Bayer, Germany). The EKOS endowave system uses ultrasound waves to enhance thrombolysis. The ultrasound waves influence the fibrin strands to make them more susceptible to plasmin and also the drug is dispersed more evenly throughout the clot. Only retrospective studies are performed using this device and there is no clear information available showing reduced treatment time in DVT.(10)(fig1a)

The trellis-8 isolates the clot between two balloons over a length of $15-30 \mathrm{~cm}$. Between the balloons a sinusoidal wire is present and after infusion of thrombolytic drugs, the wire is rotated at $3000 \mathrm{RPM}$. After around 20 minutes the solution of clot and thrombolytics can be aspirated through the same catheter. This prevents the infused thrombolytics from spreading systemically since they are infused and aspirated within the enclosed segment, further reducing the risk of bleeding. This procedure can be repeated for multiple segments in the same session. The whole procedure can take up to 4 hours in the Cath lab. For this method are also only retrospective studies available.(11) (fig 1b)

The Angiojet Power Pulse system (Bayer, Germany) is a PMT catheter that uses a complex mixture of rapid fluid streaming and hydrodynamic forces to fracture thrombus, allowing extraction at the catheter tip using negative pressure based on the so-called Bernoulli principle. The catheter infuses normal saline through an infusion port while simultaneously suctioning through the effluent port. If the effluent port is clamped, the infusion port acts as a mechanical "pulse spray system", whereby the thrombus can be laced with a thrombolytic drug that is infused through the system. This system can also be used without thrombolytics. Only retrospective studies and registries are available as evidence for this device.(12)(fig 1c) 
(a)

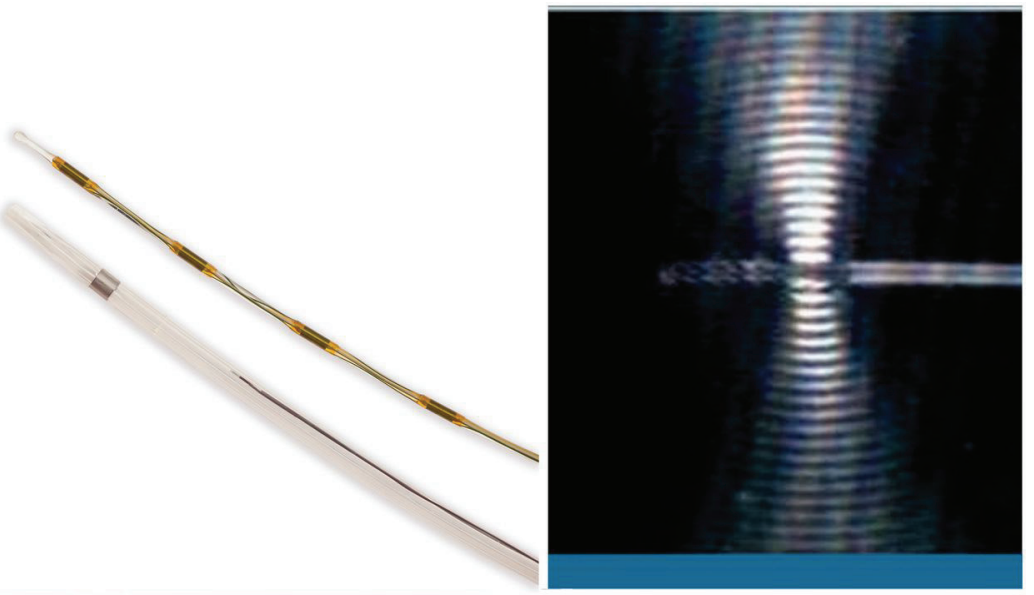

(b)
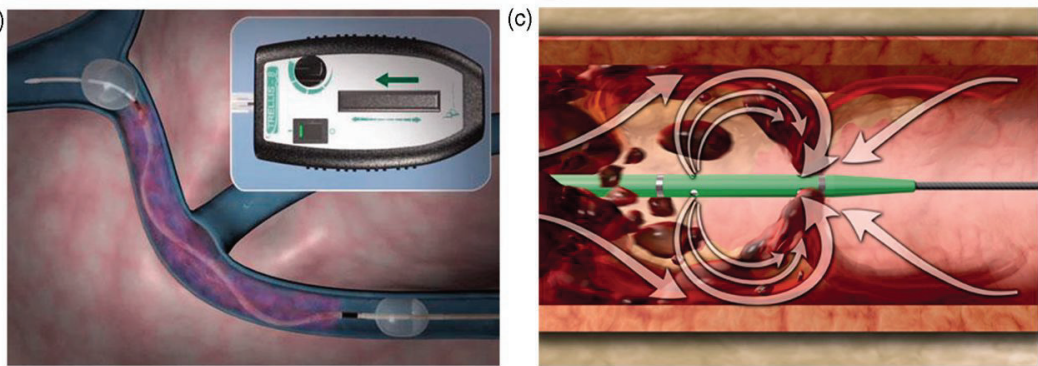

Figure 1: (a) Close-up of the EKOS $®$ endowave catheter (Reproduced with permission from EKOS ${ }^{\circledR}$ ), (b) Picture of the trellis-8 device (Reproduced with permission from Covidien), (c) picture of the angiojet powerpulse catheter (Reproduced with permission from Bayer Health Care Medical Care).

\section{Results:}

Evidence on these devices is largely derived from retrospective studies and registries. All techniques seem to be safe and feaßsible in removing thrombus. Patency rates after one year are good and there were no intracranial hemorrhages reported and no mortality. Total treatment time is not reported in most of these reports. Patients were carefully selected to undergo these procedures, minimizing bleeding risk. A summary of the available literature is shown in table 1. 
Table 1

\begin{tabular}{|c|c|c|c|c|c|c|c|}
\hline Author & Study design & Modality used & Number or patients & $\begin{array}{l}\text { Recanalisation } \\
\text { success }\end{array}$ & $\begin{array}{l}\text { Complications } \\
\text { bleeding/pulmonary } \\
\text { embolism (PE) }\end{array}$ & Follow-up length & Follow-up results \\
\hline Hilleman ${ }^{15}$ & Registry & Trellis-8 & 124 & $100 \%$ & No bleeding or PE & $\mathrm{N} / \mathrm{A}$ & N/A \\
\hline Dietzek $^{16}$ & Registry & Trellis-8 & 2203 & $100 \%$ & No bleeding or PE & N/A & N/A \\
\hline O'Sullivan ${ }^{17}$ & Retrospective & Trellis-8 & 19 & $100 \%$ & No bleeding or PE & I year & $\begin{array}{l}\text { Patency } 9 \text { out of II segments } \\
\text { were patent }\end{array}$ \\
\hline Arko $^{18}$ & Retrospective & $\begin{array}{l}\text { Trellis-8 and } \\
\text { Angiojet }\end{array}$ & $\begin{array}{l}30 \text { (Trellis 18, } \\
\text { Angiojet 12) }\end{array}$ & $100 \%$ & No bleeding or PE & 6 months & $\begin{array}{l}\text { Patency: } 90 \% \text { Preserved valve } \\
\text { function: } 88 \%\end{array}$ \\
\hline $\mathrm{Rao}^{19}$ & Retrospective & $\begin{array}{c}\text { Trellis-8 and } \\
\text { Angiojet }\end{array}$ & $\begin{array}{l}43 \text { (Trellis 13, angiojet } \\
\text { 12, both 17) }\end{array}$ & $95 \%$ & $5 \%$ bleeding, no PE & 5 months & $\begin{array}{l}\text { Reduction in symptoms: } \\
93 \% \text { of patients }\end{array}$ \\
\hline $\operatorname{Lin}^{20}$ & Retrospective & Angiojet & 49 & $100 \%$ & 4\% bleeding, no PE & I year & Patency: $68 \%$ \\
\hline Bush $^{21}$ & Retrospective & Angiojet & 20 & $100 \%$ & $15 \%$ bleeding, no PE & $N / A$ & - \\
\hline Kasirajan 22 & Retrospective & Angiojet & 17 & $100 \%$ & No bleeding or PE & II months & $\begin{array}{l}\text { Recurrence: } 52 \% \\
\text { recurrence free }\end{array}$ \\
\hline Motarjeme $\mathrm{e}^{23}$ & Retrospective & EKOS & 12 & $83 \%$ & No bleeding or PE & I year & Patency: 92\% \\
\hline Parikh $^{24}$ & Retrospective & EKOS & 32 & $91 \%$ & 3.8\% bleeding, no PE & None & - \\
\hline Grommes $^{25}$ & Retrospective & EKOS & 12 & $92 \%$ & 8\% bleeding, no PE & 7 months & Patency $92 \%$ \\
\hline Strijkers ${ }^{26}$ & Retrospective & EKOS & 37 & $95 \%$ & II\% bleeding, I PE & I year & Patency $87 \%$ \\
\hline
\end{tabular}

Drawbacks of current therapies:

Although advances were made with these new devices, there are still concerns. The major drawback of the current techniques is the usage of thrombolytics. Patients with a higher bleeding risk are excluded for these treatments because of the use of thrombolytics. Postoperative patients and patients who have active malignancies are an example of patients not eligible for most thrombolysis techniques, which makes the therapy unavailable for a large group of patients, estimated to be around $30 \%$ of all DVT's.

Duration of the patients' complaints and clot age is also of essence. A thrombus can be lysed if it is not older than 3 weeks. After three weeks the fibrin strands in the clot form tight crosslinks, and collagen is formed within the clot, which cannot be dissolved. The clot will also stick to the wall due to the inflammatory response.(13)

Bleeding risk is still an issue, even with the new PMT techniques. The patient has to be admitted into the hospital and needs monitoring during the lytic therapy. Fibrinogen levels are closely monitored and if the level drops below $1.0 \mathrm{~g} / \mathrm{l}$ thrombolytic therapy is discontinued, which again influences the outcome of the treatment. The complete process takes a couple of days, which strongly influences cost. Reduction in the length of stay in the hospital could reduce the burden of the procedure for the patient and decrease the cost of the therapy.

\section{Additional procedures}

After successful thrombolysis approximately 50\% of patients will need additional therapy. In many cases of iliofemoral DVT an obstructive lesion or compression syndrome can be identified. If left untreated this significantly increases the chance of a recurrent DVT. If a persistent obstruction is identified it has to be treated with balloon dilatation and stenting. Pneumatic compressive stockings can also improve outcome. Follow-up should be performed in a standardized fashion, which allows timely re-intervention in case of re- 
thrombosis of the treated and/or stented tract, especially in the first few weeks.

\section{Future developments:}

To address all of the previously mentioned drawbacks, new treatment techniques should adhere to a couple of preferred features. Pure mechanical removal of the clot in one session seems to be the most likely preferred treatment, ideally in a day-care setting. In short the patient would report to the physician. Standard anticoagulation treatment should be started at that point. The physician assesses eligibility for clot removal therapy and the same day the clot would be removed in an outpatient setting. Before starting the procedure a full venous roadmap should be made, using Duplex, magnetic resonance venography (MRV) or computed tomography venography (CTV) and the patient should be classified according to the LET classification (fig 2).(14) After successful thrombus removal, ideally in less then an hour via a popliteal vein approach, immediate additional stenting of a pre or per procedural identified underlying lesion should be performed. After completion of the therapy the patient is immediately discharged and continues with the standard treatment, being anticoagulation, compression and mobilization.

\section{Figure 2}
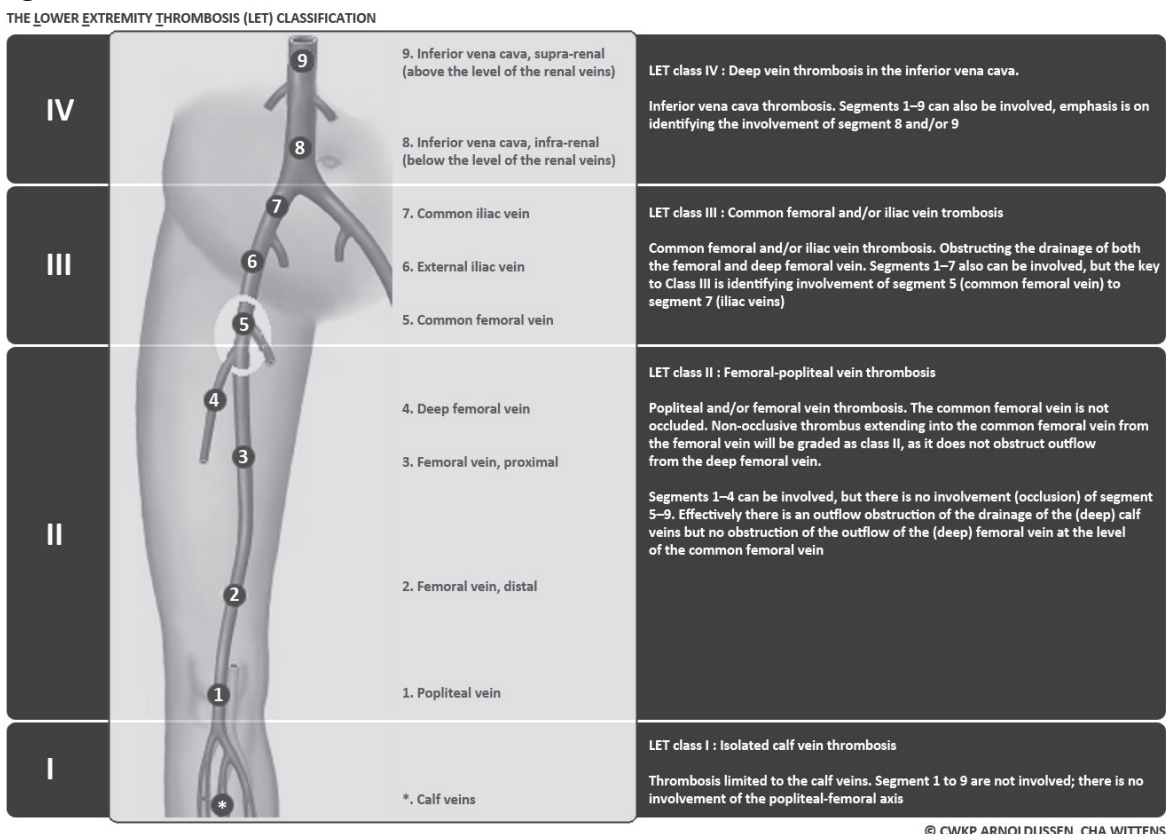
Currently available purely mechanical thrombectomy devices:

\section{Lazarus ReCover ${ }^{T M}$ :}

The Lazarus ReCover is a next-generation ischemic stroke of today's stent based retrievers. The ReCover incorporates a cover segment that automatically covers and protects the thrombus as the device is removed, helping to prevent clot embolization and secondary stroke. This device is an example of a purely mechanical clot removal therapy, but its use seems to be limited to ischemic cerebral strokes. (Fig 3)

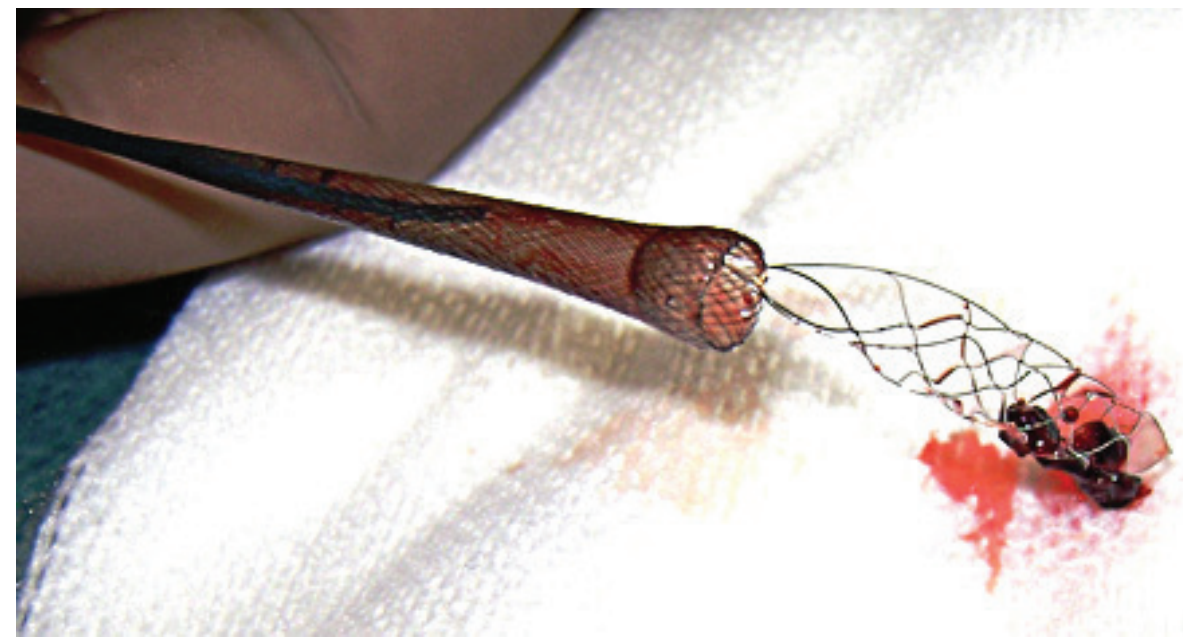

figure 3: Picture of the Lazarus device. Reproduced with permission from Lazarus Effect, Inc.

\section{AngioVac:}

The AngioVac Cannula (AngioDynamics, Latham, New York) is a mechanical suction device that is designed for removal of intravascular material such as thrombus, tumor, foreign bodies, and vegetation, while maintaining flow during extracorporeal circulation. The suction cannula is a $22-\mathrm{F}$ device that can be advanced over a wire using an internal dilator. The device has an expandable tip that opens up to 48-F. This tip serves as the suction end of a veno-venous non-oxygenating bypass circuit that filters removed blood and returns it to the venous system via a separate reinfusion cannula or sheath. The patient is put under general anaesthesia to perform this procedure. Maximum anticoagulation is given to keep the bypass circuit open and a perfusionist monitors it. This makes the procedure very invasive and not eligible for every patient. It might be an option for patients in whom initial thrombolytic therapy failed with persisting complaints. Evidence for this procedure is limited to case reports in the available literature. Therefore it is not possible to assess weather it is feasible 
and safe to use in iliofemoral and inferior vena cava DVT's and long term patency rates after follow-up are lacking.(fig 4)
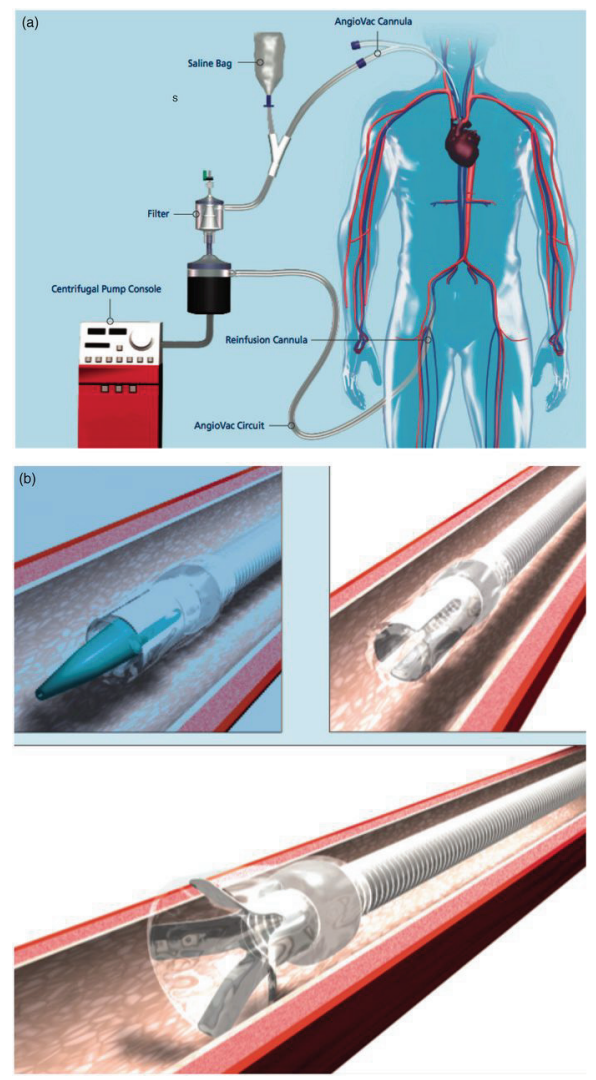

figure 4: $(\mathrm{a}, \mathrm{b})$ Picture of the AngioVac system. Reproduced with permission from AngioVac.

\section{The future of purely mechanical clot removal therapy}

New devices should preferably have the following features: Minimally invasive, fast, and with low risks of bleeding and other complications or no risks at all. Additionally the device should be cheap, available for all sorts of patient, capable of removing clot older than 3 weeks, and easy to use. These items are all limitations of the currently available therapies. We will discuss each feature below.

\section{Minimally invasive:}

Some devices are more minimally invasive than others. The Ekos endowave, Trellis-8 and Angiojet can all be used with one single entry in the popliteal vein of the patient. Less invasive is probably not possible, because entry to the ves- 
sel will always be necessary, even with future devices. The procedure can be performed in a cath lab with local anaesthesia. Contrary to this the Angiovac system requires more than 1 entry for the system to work. Besides that, the patient is under general anaesthesia when the procedure is performed. This will limited the recovery time for the patient. Ideally the patient should mobilise as soon as possible after the clot is fully removed. The available mechanical devices all have a low profile system with a limited reach and often do not clear the complete thrombus in large veins from wall to wall, such as the iliac veins with diameters up to $2 \mathrm{~cm}$. A new mechanical device should catch the complete thrombus from wall to wall and remove it all in one run.

\section{Treatment time:}

The Ekos endowave takes between 12-72 hours to fully dissolve the clot. The whole time the patient is monitored in the hospital, which brings high costs. At the same time, with the catheter in place the patient is unable to mobilise. The trellis- 8 and Angiojet do not have this problem, but therapy with the Trellis- 8 can take up to 4 hours to completely remove the clot and the Angiojet is limited in total treatment time, due to haemolysis. If the clot is not completely removed than therapy is continued with a thrombolysis catheter until the desired clot removal is established. Ideally the surgeon or interventional radiologist should perform the complete treatment in one setting within 2 hours maximum without thrombolytics.

\section{Contra-indications:}

Another advantage of purely mechanical clot removal is the reduction of bleeding risk. Because thrombolytics are not used, their inherent bleeding risk is avoided. This could potentially mean that patients who are not currently eligible for thrombolytic therapy, such as post-operative patients, pregnant women, and patients with malignancies, are potential candidates for purely mechanical clot removal. This will make clot removal therapy available to a much larger group of patients. However, bleeding risk will not be reduced to zero, because standard anticoagulation treatment will still be administered. A lot of contraindications for thrombolytic therapy at this moment will not apply for future clot removal therapy.

\section{The potential of removing older clots}

Possibly clots older than 3 weeks can be removed. Nowadays clot age is estimated according to duration of complaints. However this can be misleading and the clot could be older than was guessed based on this information. Between 3 and 6 weeks the clot becomes harder to lyse with the current treatments. Intense crosslinking between fibrin strands and the replacement of red 
blood cells and platelets by collagenous tissue gradually transforms the clot into fibrous tissue. The fibrous tissue eventually forms permanent strands within the vein wall. These cannot be dissolved or removed by thrombolytic therapy. A good mechanical device could potentially make it possible to also remove clots older than 3 weeks. What the potential window of removal will be is unclear and will become apparent in the future. Advances in imaging techniques such as MRV and CTV could prove to be useful in making an estimate whether the clot is acute, sub-acute or has already partly or completely changed into an intraluminal scar.

\section{Cost reduction}

If the mechanical therapy can be done in a single day, this could potentially save a lot of resources, due to reduced hospital stay. Reduced complications also help with cost reduction. Therefore the cost-effectiveness of new therapies should increase. Ideally the cost of catheter itself would also be reasonable to further support this cost-reduction.

\section{Easy to use}

The catheter should be easy to use, so there should be a small learning curve, enabling all involved specialists to use it. This makes it a lot easier for patients to receive the therapy in a hospital in their own region. It should also not damage vein valves, in particular when the device is introduced through the popliteal vein.

\section{Future of imaging techniques in DVT}

In cases of iliofemoral DVT, the patient should be assessed extensively by duplex ultrasound and additionally with CTV or MRV before starting a procedure. Standardizing reporting will make it possible to pool data gathered from future studies. Scorings systems, like the LET classification, based on extensive imaging, could provide physicians with an objective tool to make clinical decisions for their patients.(14)

Furthermore CTV and MRV may prove useful in determining underlying stenotic lesions in the iliac tract and inferior vena cava, which is more difficult to assess using duplex ultrasound. Stenotic lesions should not be treated with thrombolysis or clot removal, but recanalization, balloon angioplasty and stenting. Accurate pre-interventional imaging assessment to identify such lesions enables correct pre-interventional treatment selection. In the future it may be possible to assess clot age based on these imaging techniques. This could further enhance patient selection and make sure the patient receives the right clot removal strategy depending on the clot present. 
Per procedural information can also dictate if additional procedures are needed. The per-procedural information of a phlebography in different directions, an additional 3D phlebography or an intravascular ultrasound (IVUS) can also determine additional strategies. But logistically, with regard to treatment and procedure time and to limit patient en doctor exposure to radiation it is better to know it in advance.

\section{Dedicated stent material}

In order to get the best result dedicated venous stents should be used. With the right size, flexibility and radial force. Known newly dedicated venous stents are the Zilver vena (Cook)(fig.5) and the Sinus Venous (OptiMed)(fig.6)

Figure 5: Zilver vena stent. Reproduced with permission from Cook Medical.

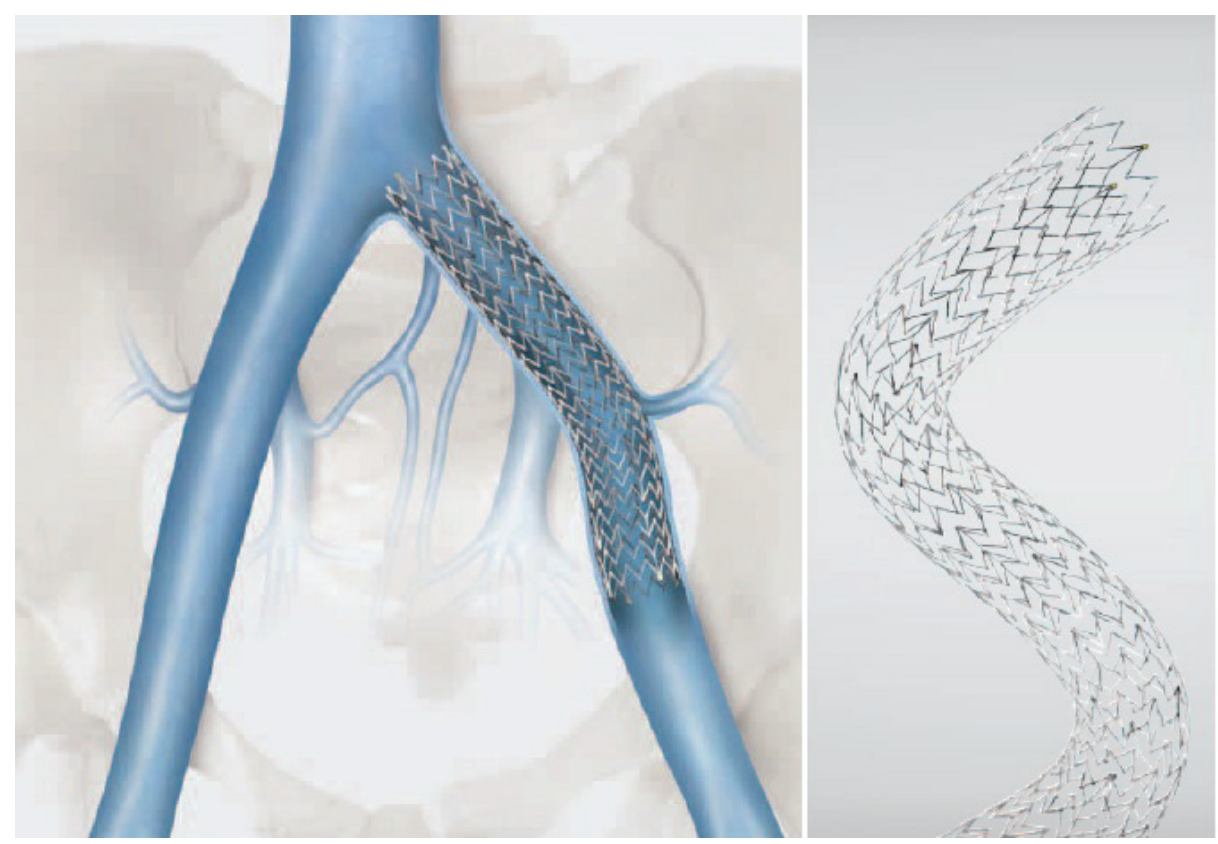

Figure 6: Close-up of the sinus venous stent. Reproduced with permission from Rembrandt Medical. 


\section{Independent Ring System}
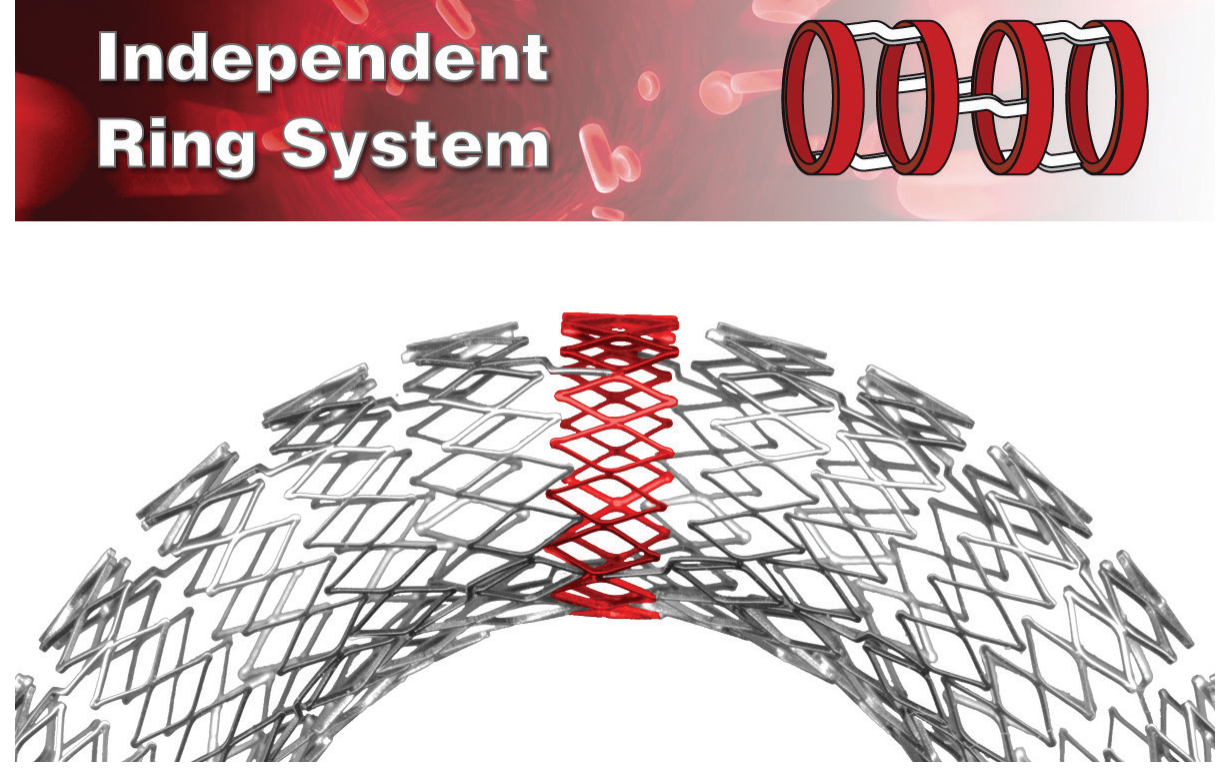

General ideas for improvement:

With enhanced awareness of the possibilities in treating patients with iliofemoral DVT we hope that physicians as well as patients will sooner evaluate, if thrombectomy, with or without stenting, should be performed. The sooner DVT gets treated the better the results. With better patient selection, more patient will be eligible to undergo therapy. With expanding knowledge, a minimally invasive non-thrombolytic outpatient treatment of an iliofemoral DVT might become the standard treatment to reduce acute symptoms, recurrence DVT and PTS incidence. If this new purely mechanical treatment seems feasible and safe even more patients might be considered for treatment. Not only in iliofemoral DVT, but perhaps also in DVT confined to the femoral or popliteal segment.

\section{Conclusion}

Clot removal therapy will most likely become standard treatment in iliofemoral deep venous thrombosis in order to prevent the post thrombotic syndrome. Currently available techniques use thrombolytic drugs with an inherent chance of bleeding and other drawbacks, like hospital admission, costs and limited patient eligibility. Future techniques will focus on purely mechanical ways of clot removal with the possible advantages of decreased bleeding risk, shorter hospital stay, lower cost and fewer contra-indications. 


\section{References:}

1. Enden T, Haig Y, Kløw N-E, Slagsvold C-E, Sandvik L, Ghanima W, et al. Long-term outcome after additional catheter-directed thrombolysis versus standard treatment for acute iliofemoral deep vein thrombosis (the CaVenT study): a randomised controlled trial. Lancet. 2012 Jan 7;379(9810):31-8.

2. Vedantham S. Catheter-directed thrombolysis for deep vein thrombosis. Curr Opin Hematol. 2010 Sep;17(5):464-8.

3. Grommes J, Strijkers RHW, Greiner A, Mahnken AH, Wittens CHA. Safety and feasibility of ultrasound-accelerated catheter-directed thrombolysis in deep vein thrombosis. European Journal of Vascular \& Endovascular Surgery. 2011 Apr;41(4):526-32.

4. Baker R, Samuels S, Benenati JF, Powell A, Uthoff H. Ultrasound-accelerated vs Standard Catheter-directed Thrombolysis-A Comparative Study in Patients with Iliofemoral Deep Vein Thrombosis. JVIR. 2012 Nov;23(11):1460-6.

5. Strijkers RHW, Cate-Hoek AJT, Bukkems SFFW, Wittens CHA. Management of deep vein thrombosis and prevention of post-thrombotic syndrome. BMJ. 2011;343:d5916.

6. Wakefield TW, Myers DD, Henke PK. Mechanisms of Venous Thrombosis and Resolution. Arteriosclerosis, Thrombosis, and Vascular Biology. 2008 Feb 22;28(3):387-91.

7. de Wolf MAF, Wittens CHA, Kahn SR. Incidence and risk factors of the post-thrombotic syndrome. Phlebology. 2012 Mar;27 Suppl 1:85-94.

8. Comerota AJ. The ATTRACT Trial: Rationale for Early Intervention for Iliofemoral DVT. Perspectives in Vascular Surgery and Endovascular Therapy. 2010 Jul 13;21(4):221-5.

9. Semba CP, Dake MD. Catheter-directed thrombolysis for iliofemoral venous thrombosis. YSVAS. 1996 Mar;9(1):26-33.

10. Strijkers RH, Grommes J, Arnoldussen CW, de Graaf R, Cate ten AJ, Wittens CH. Ultrasoundaccelerated catheter-directed thrombolysis in acute iliofemoral deep venous thrombosis. Journal of Vascular Surgery: Venous and Lymphatic Disorders. Elsevier; 2013.

11. Hilleman DE, Razavi MK. Clinical and economic evaluation of the Trellis-8 infusion catheter for deep vein thrombosis. JVIR. 2008 Mar;19(3):377-83.

12. Karthikesalingam A, Young EL, Hinchliffe RJ, Loftus IM, Thompson MM, Holt PJE. A systematic review of percutaneous mechanical thrombectomy in the treatment of deep venous thrombosis. European Journal of Vascular \& Endovascular Surgery. 2011 Apr;41(4):554-65.

13. Markel A, Meissner M, Manzo RA, Bergelin RO, Strandness DE. Deep venous thrombosis: rate of spontaneous lysis and thrombus extension. Int Angiol. 2003 Dec;22(4):376-82.

14. Arnoldussen CWKP, Wittens CHA. An imaging approach to deep vein thrombosis and the lower extremity thrombosis classification. Phlebology. 2012;27 Suppl 1:143-8.

15. Hilleman DE and Razavi MK. Clinical and economic evaluation of the Trellis-8 infusion catheter for deep vein thrombosis. JVIR 2008; 19: 377-83.

16. Dietzek AM. Isolated pharmacomechanical thrombolysis of deep venous thrombosis utilizing a peripheral infusion system: Manuf. Int Angiol 2010; 29: 308-16.

17. O'Sullivan GJ, Lohan DG, Gough N, et al. Pharmacomechanical thrombectomy of acute deep vein thrombosis with the Trellis-8 isolated thrombolysis catheter. JVIR 2007; 18: 715-24.

18. Arko FR, Davis CM, Murphy EH, et al. Aggressive percu-taneous mechanical thrombectomy of deep venous throm-bosis: early clinical results. Arch Surg 2007; 142: 513-8 (discussion 5189).

19. Rao AS, Konig G, Leers SA, et al. Pharmacomechanical thrombectomy for iliofemoral deep vein thrombosis: An alternative in patients with contraindications to thrombolysis. J Vasc Surg 2009; 50: 1092-1098.

20. Lin PH, Zhou W, Dardik A, et al. Catheter-direct throm-bolysis versus pharmacomechanical thrombectomy for treatment of symptomatic lower extremity deep venous thrombosis. The American Journal of Surgery 2006; 192: 782-8. 
21. Bush RL, Lin PH, Bates JT, et al. Pharmacomechanical thrombectomy for treatment of symptomatic lower extre-mity deep venous thrombosis: safety and feasibility study. J Vasc Surg 2004; 40: 965-70.

22. Kasirajan K, Gray B and Ouriel K. Percutaneous AngioJet thrombectomy in the management of extensive deep venous thrombosis. JVIR 2001; 12: 179-85.

23. Motarjeme A. Ultrasound-enhanced Thrombolysis. J Endovasc Ther 2007; 14: 251-6.

24. Parikh S, Motarjeme A, McNamara T, Raabe R, Hagspiel K, Benenati JF, et al. Ultrasoundaccelerated Thrombolysis for the Treatment of Deep Vein Thrombosis: Initial Clinical Experience. Journal of Vascular and Interventional Radiology 2008; 19: 521-8.

25. Grommes J, Strijkers RHW, Greiner A, et al. Safety and feasibility of ultrasound-accelerated catheter-directed thrombolysis in deep vein thrombosis. Eur J Vasc Endovasc Surg 2011; 41(4): 526-32.

26. Strijkers RH, Grommes J, Arnoldussen CW, et al. Ultrasound-accelerated catheter-directed thrombolysis in acute iliofemoral deep venous thrombosis. Journal of Vascular Surgery: Venous and Lymphatic Disorders 2013; 1: 225-230. 


\section{CHAPTER 12}

\section{Summary and general discussion}

Summary and general 


\section{Introduction}

DVT is a prevalent disease in the western world affecting approximately 1-2 per 1000 persons per year. ${ }^{1-3}$ Patients in whom DVT is suspected are selected for further diagnosis with a clinical decision rule, such as the Wells score. ${ }^{4,5}$ In combination with a d-dimer level patients are categorized as low or high probability of having DVT.6,7 If the patient is categorized as having a high probability of DVT, a two-point compression ultrasonography is typically performed to confirm or exclude the diagnosis. ${ }^{1,3,8}$ If DVT is confirmed patients are usually treated conservatively, and started on anticoagulation with low molecular weight heparin for five days in conjunction with vitamin-K-antagonists for a total duration of 3-6 months or lifelong depending on risk factors and history of previous thrombotic events. In addition compressive bandages or therapeutic elastic stockings for two years and immediate mobilization is prescribed.4, When treated accordingly, 20-50\% of all patients develop a post-thrombotic syndrome. 6,10 PTS is defined as a chronic clinical manifestations of venous hypertension following DVT.6,8 Subjective symptoms of PTS can be itching, pain, cramps, paresthesia, and heaviness of the leg. Objective symptoms can be swelling of the leg, telangiectasia, varicosities, induration, hyperpigmentation, redness or finally ulceration of the leg. Symptoms can range from mild, moderate to severe. Severe PTS is associated with increased medical and social costs, and has a large impact on the quality of life of patients, comparable to other chronic diseases like diabetes mellitus, chronic pulmonary obstructive disease and heart failure. $(9,11)$ Diagnosis of PTS is made by a clinical scale for PTS (Villalta scale) preferably in combination with a disease specific quality of life questionnaire. $(10,12)$ One in four patients with a DVT is categorized as LET 3 or 4 (iliofemoral) DVT.(6,13) A LET 3,4 DVT is associated with a higher risk of PTS. $(11,14)$

\section{Post Thrombotic Syndrome}

The exact etiology of PTS is unknown but there is a strong association with obstruction and reflux of the deep venous system.(12,15) Standard anticoagulation therapy does not actively dissolve the thrombus. The individual patients' lytic system is responsible for lysing the thrombus and thus establishing recanalization of the vein. If recanalization of the vein is not achieved, the remaining thrombus is transformed into fibrous tissue and this process results in partial or total obstruction of the vein and vein wall stiffness.(13,16) Concurrent processes of inflammation, can damage the valves, leading to venous insufficiency. $(14,17)$ These mechanisms eventually lead to venous hypertension and are considered the cause of PTS.(1,15) PTS is the most frequently occurring complication after DVT. With a reported incidence after DVT of up to 50\%, it is important to recognize patients who are at increased risk of developing PTS 
and offer them alternative therapies capable of reducing post thrombotic morbidity. Prevention of PTS could be achieved with early thrombus removal by UACDT as this could minimize obstruction of the vein and preserve the valve function. $(16,18,19)$

\section{Diagnosis and treatment of DVT}

Chapter two gives an overview of the treatment currently available to patients with iliofemoral DVT and the results regarding this treatment. It shortly introduces the problem of PTS after DVT, especially in LET 3,4, iliofemoral DVT. Physicians can use the Wells criteria combined with a d-dimer level to assess the patients risk for DVT. This is generally regarded as the standard clinical appraisal to reduce the number of unnecessary ultrasounds. The combination of a low wells score in combination with low d-dimer level has a 97.7\% negative predictive value, which makes it very useful in safely ruling out DVT. $(17,20)$ Diagnosis of DVT with two-point compression ultrasound is considered the current standard and has a sensitivity of $98.7 \%$ and specificity of $100 \%$ for above the knee thrombus and $85.2 \%$ sensitivity and $98.2 \%$ specificity for below the knee thrombus compared to the gold standard (invasive venography).(1,21) Magnetic resonance venography or computed tomography venography can be considered for DVT above the inguinal level.(18,19,22)

The risk of PTS in DVT patients varies greatly in literature from $20 \%$ to greater than $60 \%$ in LET 3,4 DVT. The risk of developing a venous ulcer after DVT ranges from 3-5\%.(20,23,24) Additional thrombus removal therapies have shown potential for regaining patency and lowering PTS. However evidence is limited to retrospective analysis and case reports.

\section{Villalta scale to diagnose PTS}

Diagnosis of PTS is discussed in Chapter three. The Villalta scale for PTS, its goals and limitations are presented. PTS consists of both patient reported symptoms and clinical signs. Symptoms include pain, heaviness, swelling, itching, cramps or tingling in the affected Leg. Symptoms can be persistent or intermittent. Exercise tends to aggravate symptoms and rest and elevation of the leg reduce symptoms.(21,25) Clinical signs include teleangiectasia, eczema, varicose veins, edema, lipodermatosclerosis and ulceration. As a result of the wide range of clinical presentations, it has proven difficult to achieve consensus on a clear definition of PTS. PTS is a syndrome and there is no gold standard diagnostic test. An ad hoc conference organized by the European Venous Forum defined PTS as chronic venous symptoms/signs secondary to DVT. $(22,26)$ In the past 20 years, a few clinical scales have been introduced to diagnose PTS. PTS is considered when a patient has had a DVT and has complaints in the ipsilateral leg that have developed or persisted a few months after DVT. Ideally, a 
clinical scale should be able to assess whether PTS is present and preferably also distinguish between severe and milder forms of PTS. The scale should be easy to use with high inter- and intra-observer reliability, so that scores are reproducible and can be used for clinical research and daily practice. The scale should be accepted and used worldwide, so that pooling of results is possible and the strongest evidence becomes available. The Brandjes, Ginsberg, and Villalta scales are specific PTS scales and include clinical sings as wells as patient symptoms. Of these scores the Villalta scale is considered as the scale of choice combining all above mentioned properties and in addition has a good correlation to generic quality of life scores (SF-36) as well as disease specific (VEINES-QOL) scores. $(23,24,27)$ The reproducibility and correlation with patients' perception of interference with life is good. Inter-rater reliability between physicians and nurses was shown to be excellent. Inter rater reliability was good for the cut-off point no PTS/PTS ( $\kappa=0.71)$, and excellent for the cutoff moderate/severe $(\kappa=1.00)$. Limitations of the scale included; lack of intrarater reliability, test-retest properties and the lack of venous claudication as a symptom and the lack of grading for venous ulceration.

Selection of patients eligible for ultrasound accelerated catheter directed thrombolysis (UACDT) treatment

Patient selection is crucial to determine who is eligible for thrombolytic treatment and who will benefit the most. Typical parameters such as thrombus load, duration of complaints and bleeding risk are taken into account. PTS incidence after calf DVT is lower compared to iliofemoral (LET 3,4) DVT.(25,28) Chapter four evaluates two different methods of use of the LET classification and its effect on predicting PTS in the long term.

The LET classification classifies patients in four different groups based on the thrombus location in the acute phase. $(19,26)$ In Chapter five we studied the properties of the LET classification in predicting PTS incidence and severity and quality of life impairment in patients. We used the exact location of the thrombus determined in the acute phase in 1338 patients of a cohort in Antwerp. Patients who died or surpassed the age of 80 years at follow-up were excluded from the analysis. In total 660 patients were invited to fill-out a questionnaire regarding their complaints. We used a modified version of the Villalta scale to assess PTS. General quality of life (SF-36) and specific quality of life (VEINES-sym/qol) were recorded as well as baseline characteristics such as age, BMI, gender, original treatment in the acute phase. Confounding factors such as: recurrent DVT, compression therapy, anticoagulation, orthopedic disease, peripheral arterial disease and length of follow-up were recorded. We correlated the initial localization of the thrombus, using the LET classification, with the patient complaints, and prevalence of PTS, and quality of life. Of the 
total of 660 patients, 315 responded. One hundred were classified as LET I, 108 as LET II and 107 as LET III. Severe PTS was reported in $18.4 \%$ of patients. Univariate analysis resulted in LET I and LET II the OR for severe PTS was 2.0(0.9-4.6) and comparing LET I and LET III resulted in an OR of 3.0 (1.4-6.7). According to multivariate logistic regression analysis LET II predicted development of severe PTS compared to LET I, with an OR of 1.9 (0.7-4.9), LET III had an OR of 3.4 (1.3-8.8) for predicting severe PTS, compared to LET I. VEINES-qol scores significantly decreased with higher LET $(p=0.003)$. The LET classification showed a good predictive value for severe PTS and decreased quality of life in DVT patients based on initial thrombus location and extension. The LET classification could prove useful in selecting patients who would benefit most of additional treatment with UACDT.

\section{Contrast enhanced-MR-Venography for acute DVT}

In order for UACDT to be successful it is important to select patients in the acute phase of thrombosis. However, symptoms can be masked, leading to a delayed presentation. This can result in unsuccessful UACDT, because of older thrombus age and processes of thrombus organization. Adequate assessment of thrombus age can potentially reduce the number of treatment failures and reduce the risks associated with thrombolysis. Thrombus age is currently determined based on patient's complaints. $(27,29,30)$ Other methods like duplex ultrasound also have been described in literature.(28,31) MR-venography (MRV) is a relatively new imaging modality and could potentially be useful for the assessment of thrombus age.(19,32-34) Chapter six describes results on the use of contrast enhanced-MRV for acute DVT. In total 46 CE-MRV's were assessed by four different observers. Two observers had great experience with MRV and venous pathology; the remaining two observers were radiologists with great experience in interpreting MRI scans, but with less experience in venous pathology. All CE-MRV scans were interpreted at the level of the common femoral vein on the affected side. Five possible options for interpretation of thrombus age were given: Acute, sub-acute, old, acute on chronic and inconclusive. Each of these interpretations was predefined by characterizations in an illustration. Agreement between the two observers with experience in venous pathology was excellent with a kappa of $0.969(\mathrm{p}<0.001)$. Agreement between the first interpreter and the other two MRI experts was also excellent with a kappa of $0.824(\mathrm{p}<0.001)$ and $0.821(\mathrm{p}<0.001)$. The interpretations of acute, sub-acute, and older thrombus age were also correlated to actual patient's complaints in days. Thrombus on CE-MRV characterized as acute had an average of 6.5 days of patient complaints (range 2-13 days), sub-acute 13 days (range 8-18 days) and old 22 days (range 15-32). This study shows that identification of specific characteristics of thrombus on CE-MRV is feasible and has a 
good to excellent inter-observer agreement. The thrombus characteristics on MRV in addition showed an adequate correlation with regards to duration of DVT complaints of the patients. CE-MRV could become a valuable diagnostic tool for DVT above the inguinal ligament.

\section{Safety and feasibility of UACDT}

CDT has been used for treatment of IFDVT and reports show successful lysis in the majority of patients. $(29,30,33,35)$ However treatment time with a median of 2.4 days is considered too long.(31,36) UACDT is designed to be more effective in quickly lysing the thrombus in patients with arterial occlusion, bypass occlusions, pulmonary embolism and DVT.(32-34,37) In Chapter seven our experience using UACDT for acute iliofemoral DVT is reported. A retrospective analysis was performed on UACDT treatments for iliofemoral DVT from October 2008 till January 2010. Twelve patients underwent UACDT for this indication. Seven patients were male and five female. Median age was 44 years (range 5-79). Diagnosis of IFDVT was made with a combination of duplex and CTvenography or duplex and MR-venography. Seven patients had poplitealfemoral-ilio-caval (LET 2,3,4) thrombsis, three had popliteal-femoral-iliac (LET 2,3 ) thrombosis, one had popliteal-femoral (LET 2) thrombosis and one patient had superior caval (LET 4) thrombosis. UACDT was successful in 85\% (11/13) with complete lysis (>90\%) in ten patients and partial lysis (50-90\%) in one. No PE was encountered and one bleeding at the catheter insertion site. In three patients, underlying residual stenosis was treated successfully with balloon angioplasty and stent placement. Four patients developed an early recurrence due to untreated residual venous obstruction.

Chapter eight reports on a larger cohort of patients treated with UACDT for acute iliofemoral (LET 3/4) DVT. In this retrospective analysis we report on 37 patients with a mean age of 42 years (range 5-76) treated in Aachen and Maastricht. In twenty cases the DVT was located on the left side, in 13 cases on the right side and four patients had bilateral DVT. Eighteen patients were male and nineteen female. Most patients had duration of complaints between 7 and 13 days. One DVT was located in popliteal-femoral (LET 2) segment, 32 patients had an iliofemoral (LET 2,3) DVT and 4 patients had also involvement of the inferior caval vein (LET 2,3,4), causing bilateral DVT. Technical success of the treatment showed Grade 1 lysis ( $>90 \%$ ) was achieved in $52 \%$ of patients; grade 2 lysis (50-90\%) was achieved in 43\%. The remaining 5\% did not show any improvement during UACDT and treatment in these patients was discontinued. Average treatment time was 43 hours till successful lysis. In $75 \%$ of left sided DVT, May-Thurner syndrome was encountered. Two patients had an inferior vena cava atresia. In total $54 \%$ of patients received additional stenting for underlying residual stenosis. Additional interventions included PTA and stenting, 
creation of an arterio-venous fistula and one open surgical thrombectomy. Primary patency after one-year follow-up was $70 \%$ as calculated with a Kaplan-Meier survival analysis. Secondary patency was $87 \%$ at one year. Regarding safety we reported $8 \%$ minor bleeding, 3\% major bleeding, 5\% other complications, including heparin-induced thrombocytopenia (HIT). We encountered one PE and none of the patients died. The PE occurred two weeks after UACDT treatment and was regarded as unrelated to UACDT. Duration of UACDT was longer than previously reported by Motarjeme and Raabe.(33,35) This observation could be explained by the different selection of patients, logistics of monitoring during treatment and differences in thrombus age. The second important observation is the high number of additional treatment needed to resolve underling residual stenosis. In more than $50 \%$ of cases additional stents were placed in the venous tract. Overall UACDT was considered feasible and safe for the treatment of acute iliofemoral DVT. The safety and feasibility of UACDT should also be investigated in methodologically rigorous trials, to better control for confounding. The CaVenT-study a randomized controlled trial, showed an absolute risk reduction in PTS with additional CDT in patients with acute DVT. However, concerns regarding bleeding risk and the potential benefit in reducing PTS remain. The CAVA trail is a randomized controlled study comparing conventional treatment and UACDT for the prevention of PTS after DVT in patients with acute DVT (complaints $<21$ days). This trial is currently enrolling patients with (LET 3/4) iliofemoral DVT in the Maastricht university medical center+ and affiliated hospitals in the Netherlands.

Another randomized controlled trial studying the effect of CDT in acute iliofemoral $($ LET3,4) DVT to reduce PTS, the Attract trial, is also underway in the United States and reports are expected in 2016.(36)The combined results of the three randomized controlled trials, should provide a definitive answer on the subject of CDT in iliofemoral DVT for the reduction of PTS.

\section{Stenting and UACDT}

In the two previous chapters a 50\% rate of stenting was reported after UACDT. Stent occlusions were encountered in a small number of these patients. Most of the patient experienced acute symptoms of leg swelling and pain. With duplex ultrasound the diagnosis of stent occlusion could be made. Because of the presence of acute thrombus an attempt was made to regain patency in these patients. In the literature there are only few reports on stent related problems. Chapter nine reports on the findings of a retrospective analysis of eighteen patients treated with acute stent occlusion between January 2009 and July 2014. All patients underwent UACDT as primary treatment. Median age was 43 years, and $67 \%$ were male. Indications for initial stenting were; treatment of chronic venous obstructive disease in 12 patients and treatment of underlying 
obstruction after initial UACDT in acute DVT in 6 patients. Technical success was achieved in 61\% (11/18). Primary patency at last follow-up was $73 \%$ (8/11), during median follow-up of 14 months (range 0-41 months). After successful lysis, 7 patients were re-stented with an arteriovenous-fistula in 6. Median lysis time of successfully lysed cases was 19 hours (range: 11-101). These results are more in line with earlier reports of UACDT. Compared to the reported treatment time in Chapter eight, UACDT duration is halved. In earlier cases lysis treatment was prolonged as result of persistent thrombus, which turned out to be residual stenosis. With increasing treatment experience recognition of these residual lesions and their resistance to therapy, was recognized and treatment would be advanced from UACDT to PTA and stenting. Patient reported duration of complaints was not useful to determine success; thrombus age was often older than reported duration of complaints would suggest. With respect to safety of the procedure: 6 complications were encountered, two minor bleeding, one clinically relevant bleeding. One HIT was encountered, and one allergic reaction to iodine contrast fluid. No PE and no deaths were encountered and overall UACDT was considered to be safe. Stent related problems were recognized as the main reason for stents occlusion, underlining the need for dedicated venous stents. If patency could be regained it should be followed directly by adequate treatment of the underlying problem. This leads to an acceptable one-year patency of 73\%. A follow-up regimen after stent placement is proposed to ensure that problems can be recognized in an early stage of reocclusion when UACDT is still possible. Follow-up at intervals of initially two weeks, six weeks, three months, six months, and one year should be sufficient to catch any potential occlusion or stent failure. This regimen should be prospectively evaluated for effectiveness.

\section{Safety during UACDT}

Frequent blood monitoring is performed In order to ensure the safety of patients undergoing UACDT. Fibrinogen levels should not drop below 1.0g/l as this increases the chance of bleeding anywhere in the body. In case of fibrinogen levels below 1.0g/l we supplement plasma fibrinogen by giving Fresh Frozen Plasma (FFP). In chapter ten we compared three different methods of determining plasma fibrinogen levels in patients undergoing UACDT. The most recommended and used one is the Clauss method. Both optic and mechanical clot detection methods are accepted as standard methods and deemed equal. ROTEM FIBTEM is an alternative method of determining fibrinogen levels in patients. However this method is not considered standard. We noticed difficulties determining fibrinogen levels in patients undergoing UACDT. Optic measurement by CS2100i, mechanical measurement by STA-go, and functional measurement by ROTEM were compared in this study. Fifteen patients under- 
going UACDT were prospectively included and blood samples were analyzed with all three techniques. A mean difference of $0.5 \mathrm{~g} / \mathrm{l}$ in plasma fibrinogen level for all samples (95\% confidence interval (95\% CI): 0.05-0.96) was found between the optical and mechanical method. Besides the difference in we also found a large number of samples, which returned with a coagulation curve error. This occurred in $41 \%$ of all samples using optical measuring, 2\% with mechanical measuring and no curve errors with ROTEM FIBTEM. D-dimer has been suggested in literature as potential disturbing factor for fibrinogen levels. We compared d-dimer levels in samples with and without curve errors. The samples without coagulation curve error $(n=49)$ had a median d-dimer level of $54 \mu \mathrm{g} / \mathrm{l}$ (IQR 20.0-91.3) and 50 $\mathrm{gg} / \mathrm{l}$ (27.5-118.5) for the group with coagulation curve error $(n=35)$. D-dimer levels did not differ significantly between the groups ( $p=0.655)$. A large number of FFP's was given to patients (30 in total), because coagulation curve error samples were regarded as fibrinogen levels dropping below $1.0 \mathrm{~g} / \mathrm{l}$. In two cases minor bleeding was observed and no major bleeding occurred. The question remains what causes the discrepancy between the different techniques and which technique produces the most reliable results in terms of safety for the patient. Based on our results we assume that cs2100i underestimates fibrinogen levels. High D-dimer levels did not correlate with errors in our study.

\section{Future research}

Chapter eleven gives an overview of the treatment modalities currently available for interventional treatment of acute iliofemoral DVT and also shows a perspective on the advances that can be made in the near future. UACDT is one of the options for removing thrombus in an acute phase of DVT. However, there are advances in the technique of thrombus removal. Additional thrombus removal techniques should be used to lower the incidence of PTS incidence. In order to maintain an optimal benefit/harm ratio the used technique should be safe. Most of the current techniques use thrombolytic drugs to dissolve the thrombus.(37) This carries an inherent risk of bleeding, which can be potentially devastating for the patient if it occurs in a critical organ (e.g. intracranial, retroperitoneal, and intra-ocular). Pure mechanical thrombectomy devices do not use thrombolytic drugs and thus the inherent bleeding risk is not present. Patients who have contra-indications for the current techniques can potentially be treated with those newer techniques, such as postoperative DVT's or DVT in patients with cancer (total about $30 \%$ of all DVT's). Current thrombectomy devices are not capable of removing the full thrombus load in acute iliofemoral (LET 3/4) DVT. Future imaging techniques could be used to assess thrombus age and diagnose underlying stenosis making better patient selection possible. 
Improvement in patient selection is necessary to ensure thrombolysis is possible, and can be effective in reducing PTS.

Most of the materials currently used are directly adapted from the techniques available for the arterial system. $(5,38)$ The characteristics of the venous vasculature differ significantly from the arterial vasculature. This makes it necessary to design dedicated materials for use in the venous system. Dedicated venous stents may decrease the number of stent related problems and make adequate treatment of underlying stenotic lesions possible more often.

Future thrombectomy devices should have a minimal invasive character, have the potential to remove the complete thrombus load, and should be easy to use. The treatment should be fast and allow for immediate stenting if necessary. And also of practical importance towards implementation, treatment should be cost effective compared to current treatment. With the suggested advances in the techniques it could well be possible to not only treat acute (LET 3,4) iliofemoral DVT, but also include popliteal-femoral (LET 2) DVT and somewhat older thrombus.

\section{Conclusion and future perspective}

In conclusion, this thesis discusses the invasive treatment options for the prevention of PTS after DVT. Especially iliofemoral DVT (LET III) causes severe PTS. UACDT in the acute phase could potentially lower PTS. This thesis shows the safety and feasibility of UACDT in acute LET 3,4 iliofemoral DVT and in stent thrombosis. There is a need for better identification of patients at risk for PTS after DVT. The LET classification could be an easy tool for radiologist or other physicians to categories patients in high-risk group for developing PTS. To ensure successful treatment it is necessary to assess thrombus age and feasibility of UACDT. MR-venography showed the potential for adequately diagnosing acute thrombosis with good inter-rater agreement. Better patient selection, non-thrombolytic stenting techniques, and dedicated stenting materials for the venous system can enhance feasibility of UACDT. Although the CaVenTstudy showed an absolute risk reduction in PTS with additional CDT in patients with acute DVT, there are still concerns. These concerns regard bleeding risk and the potential benefit in reducing PTS. The combined results of three randomized controlled trials (two trials currently enrolling patients), should give a definitive answer on the subject of CDT in (LET 3,4) iliofemoral DVT for the reduction of PTS. Anticoagulation treatment for DVT is rapidly changing. Alternatives for the oral vitamin K-antagonists such as Dabigatran, Rivaroxaban, and Apixaban are more frequently used in daily practice. $(39,40)$ A potential beneficial effect of these new anticoagulation agents is hypothesized, however an 
effect on PTS reduction is to be seen. Future research should look into the working mechanism of these agents on the development of PTS. Routine use of compression stockings was regarded as standard since the 90's. However fairly recently, the role of therapeutic elastic compression stockings (TECS) was reevaluated in a randomized controlled, which showed no additional benefit of wearing TECS for 2 years on PTS incidence.(41) Further research should focus on the working principle of TECS and the indication and duration of wearing TECS to prevent PTS. As the call for preventing PTS becomes louder and the possibilities of early thrombus removal increase, it can be expected that early thrombus removal therapies will become standard in the near future. At the moment only a select group of patients qualifies for these procedures, but with increasing safety and awareness of the benefits more patients will be eligible. The potentially large group of patients with treatable DVT should make manufacturers aware of the possibilities of investing in treatment options. If industry and health professionals combine their efforts in this matter it is to be expected that major improvements can be made in the near future.

DVT diagnosis and treatment is divided amongst specialists. Treatment is taken care of mostly taken care of by internists. Radiologists play an important role in diagnosing DVT, dermatologists can suggest adequate compression therapy and in acute severe cases vascular surgeons in combination with interventional radiologist can provide adequate care. With help of the LET classification we hope to simplify the treatment algorithm for DVT in such a manner that general practitioners are able to determine if the patients needs to see a specialist directly after DVT diagnosis. In mild cases the general practitioner can coordinate all the care of the patient and in more severe cases the general practitioner will refer to a specialized venous center for adequate anticoagulation therapy and thrombus removal therapy. 


\section{References:}

1. Satin R, Tousignant L, Sheiner N. A prospective study comparing duplex scan and venography for diagnosis of lower-extremity deep vein thrombosis. Cardiovascular Surgery. 1996.

2. Nordström M, Lindblad B, Bergqvist D, Kjellström T. A prospective study of the incidence of deep-vein thrombosis within a defined urban population. J Intern Med. 1992 Aug;232(2):15560.

3. Bates SM, Jaeschke R, Stevens SM, Goodacre S, Wells PS, Stevenson MD, et al. Diagnosis of DVT: Antithrombotic Therapy and Prevention of Thrombosis, 9th ed: American College of Chest Physicians Evidence-Based Clinical Practice Guidelines. Chest. 2012 Feb 6;141(2 suppl):e351Se418S.

4. Kearon C, Akl EA, Comerota AJ, Prandoni P, Bounameaux H, Goldhaber SZ, et al. Antithrombotic therapy for VTE disease: Antithrombotic Therapy and Prevention of Thrombosis, 9th ed: American College of Chest Physicians Evidence-Based Clinical Practice Guidelines. Chest. 2012 Feb;141(2 Suppl):e419S-94S.

5. Wells PS, Anderson DR, Rodger M, Forgie M, Kearon C, Dreyer J, et al. Evaluation of D-dimer in the diagnosis of suspected deep-vein thrombosis. N Engl J Med. 2003 Sep 25;349(13):1227-35.

6. Kahn SR, Shrier I, Julian JA, Ducruet T, Arsenault L, Miron MJ, et al. Determinants and time course of the postthrombotic syndrome after acute deep venous thrombosis. Ann Intern Med. 2008 Nov 18;149(10):698-707.

7. Engelberger RP, Aujesky D, Calanca L, Staeger P, Hugli O, Mazzolai L. Comparison of the diagnostic performance of the original and modified Wells score in inpatients and outpatients with suspected deep vein thrombosis. Thrombosis Research. Elsevier Ltd; 2011 Jun 1;127(6):535-9.

8. Kahn SR, Partsch H, VEDANTHAM S, Prandoni P, Kearon C, Subcommittee on Control of Anticoagulation of the Scientific and Standardization Committee of the International Society on Thrombosis and Haemostasis. Definition of post-thrombotic syndrome of the leg for use in clinical investigations: a recommendation for standardization. Journal of Thrombosis and Haemostasis. 2009 May;7(5):879-83.

9. Kahn SR, Shbaklo H, Lamping DL, Holcroft CA, Shrier I, Miron MJ, et al. Determinants of healthrelated quality of life during the 2 years following deep vein thrombosis. Journal of Thrombosis and Haemostasis. 2008 Jul;6(7):1105-12.

10. Baldwin MJ, Moore HM, Rudarakanchana N, Gohel M, Davies AH. Post-thrombotic syndrome: a clinical review. Journal of Thrombosis and Haemostasis. 2013 May;11(5):795-805.

11. Tick LW, Kramer MHH, Rosendaal FR, Faber WR, Doggen CJM. Risk factors for post-thrombotic syndrome in patients with a first deep venous thrombosis. Journal of Thrombosis and Haemostasis. 2008 Dec;6(12):2075-81.

12. Ziegler S, Schillinger M, Maca TH, Minar E. Post-thrombotic syndrome after primary event of deep venous thrombosis 10 to 20 years ago. Thrombosis Research. 2001 Jan 15;101(2):23-33.

13. Tick LW, Doggen CJM, Rosendaal FR, Faber WR, Bousema MT, Mackaay AJC, et al. Predictors of the post-thrombotic syndrome with non-invasive venous examinations in patients 6 weeks after a first episode of deep vein thrombosis. Journal of Thrombosis and Haemostasis. 2010 Dec;8(12):2685-92.

14. Roumen-Klappe EM, Janssen MCH, van Rossum J, Holewijn S, Van Bokhoven MMJA, Kaasjager K, et al. Inflammation in deep vein thrombosis and the development of post-thrombotic syndrome: a prospective study. Journal of Thrombosis and Haemostasis. 2009 Apr;7(4):582-7.

15. Pascarella L, Penn A, Schmid-Schonbein GW. Venous Hypertension and the Inflammatory Cascade: Major Manifestations and Trigger Mechanisms. Angiology. 2005 Nov 1;56(6 suppl):S3-S10.

16. Elsharawy M, Elzayat E. Early results of thrombolysis vs anticoagulation in iliofemoral venous thrombosis. A randomised clinical trial. European Journal of Vascular \& Endovascular Surgery. 2002 Sep;24(3):209-14. 
17. Linkins LA, Bates SM, Ginsberg JS, Kearon C. Use of different D-dimer levels to exclude venous thromboembolism depending on clinical pretest probability. Journal of Thrombosis and Haemostasis. 2004 Aug;2(8):1256-60.

18. Baldt MM, Zontsich T, Stümpflen A, Fleischmann D, Schneider B, Minar E, et al. Deep venous thrombosis of the lower extremity: efficacy of spiral CT venography compared with conventional venography in diagnosis. Radiology. 1996 Aug;200(2):423-8.

19. van Langevelde K, Tan M, Šrámek A, Huisman MV, de Roos A. Magnetic resonance imaging and computed tomography developments in imaging of venous thromboembolism. J Magn Reson Imaging. 2010 Nov 23;32(6):1302-12.

20. van Gent WB, Wilschut ED, Wittens C. Management of venous ulcer disease. BMJ. 2010;341:c6045.

21. Kahn SR, Hirsch A, Shrier I. Effect of postthrombotic syndrome on health-related quality of life after deep venous thrombosis. Arch Intern Med. 2002 May 27;162(10):1144-8.

22. Eklof B, Perrin M, Delis KT, Rutherford RB, Gloviczki P, American Venous Forum, et al. Updated terminology of chronic venous disorders: the VEIN-TERM transatlantic interdisciplinary consensus document. Journal of vascular surgery : official publication, the Society for Vascular Surgery [and] International Society for Cardiovascular Surgery, North American Chapter. 2009. pp. 498-501.

23. Villalta S, Bagatella P, Piccioli A, Lensing A, Prins M, Prandoni P. Assessment of validity and reproducibility of a clinical scale for the post-thrombotic syndrome. Haemostasis; 1994;24(158a).

24. Kahn SR, Desmarais S, Ducruet T, Arsenault L, Ginsberg JS. Comparison of the Villalta and Ginsberg clinical scales to diagnose the post-thrombotic syndrome: correlation with patientreported disease burden and venous valvular reflux. Journal of Thrombosis and Haemostasis. 2006 Apr; 4(4):907-8.

25. Kahn SR, Kearon C, JULIAN JA, MacKinnon B, Kovacs MJ, WELLS P, et al. Predictors of the postthrombotic syndrome during long-term treatment of proximal deep vein thrombosis. Journal of Thrombosis and Haemostasis. 2005 Apr;3(4):718-23.

26. Arnoldussen CWKP, Wittens CHA. An imaging approach to deep vein thrombosis and the lower extremity thrombosis classification. Phlebology. 2012;27 Suppl 1:143-8.

27. Vedantham S, Grassi CJ, Ferral H, Patel NH, Thorpe PE, Antonacci VP, et al. Reporting standards for endovascular treatment of lower extremity deep vein thrombosis. JVIR. 2009 Jul;20(7 Suppl):S391-408.

28. Gornik HL, Sharma AM. Duplex ultrasound in the diagnosis of lower-extremity deep venous thrombosis. Circulation. 2014 Feb 25;129(8):917-21.

29. Vik A, Holme PA, Singh K, Dorenberg E, Nordhus KC, Kumar S, et al. Catheter-directed thrombolysis for treatment of deep venous thrombosis in the upper extremities. Cardiovasc Intervent Radiol. 2009 Sep;32(5):980-7.

30. Vedantham S. Catheter-directed thrombolysis for deep vein thrombosis. Curr Opin Hematol. 2010 Sep;17(5):464-8.

31. Enden T, Haig Y, Kløw N-E, Slagsvold C-E, Sandvik L, Ghanima W, et al. Long-term outcome after additional catheter-directed thrombolysis versus standard treatment for acute iliofemoral deep vein thrombosis (the CaVenT study): a randomised controlled trial. Lancet. 2012 Jan 7;379(9810):31-8.

32. Parikh S, Motarjeme A, McNamara T, Raabe R, Hagspiel K, Benenati JF, et al. Ultrasoundaccelerated Thrombolysis for the Treatment of Deep Vein Thrombosis: Initial Clinical Experience. Journal of Vascular and Interventional Radiology. 2008 Apr;19(4):521-8.

33. Raabe RD. Ultrasound-accelerated Thrombolysis in Arterial and Venous Peripheral Occlusions: Fibrinogen Level Effects. JVIR. Elsevier Inc; 2010 Aug 1;21(8):1165-72.

34. Engelhardt TC, Taylor AJ, Simprini LA, Kucher N. Catheter-directed ultrasound-accelerated thrombolysis for the treatment of acute pulmonary embolism. Thrombosis Research. 2011 Aug;128(2):149-54. 
35. Motarjeme A. Ultrasound-enhanced Thrombolysis. J Endovasc Ther. 2007 Apr;14(2):251-6.

36. Comerota AJ. The ATTRACT Trial: Rationale for Early Intervention for Iliofemoral DVT. Perspectives in Vascular Surgery and Endovascular Therapy. 2010 Jul 13;21(4):221-5.

37. Karthikesalingam A, Young EL, Hinchliffe RJ, Loftus IM, Thompson MM, Holt PJE. A systematic review of percutaneous mechanical thrombectomy in the treatment of deep venous thrombosis. European Journal of Vascular \& Endovascular Surgery. 2011 Apr;41(4):554-65.

38. Kwak H-S, Han Y-M, Lee Y-S, Jin G-Y, Chung G-H. Stents in common iliac vein obstruction with acute ipsilateral deep venous thrombosis: early and late results. JVIR. 2005 Jun;16(6):815-22.

39. Mantha S, Ansell J. Indirect comparison of dabigatran, rivaroxaban, apixaban and edoxaban for the treatment of acute venous thromboembolism. J Thromb Thrombolysis. 2014 Jul 3.

40. EINSTEIN Investigators, Bauersachs R, Berkowitz SD, Brenner B, Büller HR, Decousus H, et al. Oral rivaroxaban for symptomatic venous thromboembolism. N Engl J Med. 2010 Dec 23;363(26):2499-510.

41. Kahn SR, Shapiro S, Wells PS, Rodger MA, Kovacs MJ, Anderson DR, et al. Compression stockings to prevent post-thrombotic syndrome: a randomised placebo-controlled trial. Lancet. 2014 Mar 8;383(9920):880-8. 


\section{Nederlandse samenvatting}

Nederlandse samenvatting

Nederlandse 
DVT is een veelvoorkomende aandoening in de westerse wereld. De incidentie wordt geschat op ongeveer 1-2 per 1000 inwoners per jaar. Patiënten bij wie het vermoeden op een DVT bestaat worden voorgeselecteerd met een klinische beslisregel zoals de Wells score. In combinatie met een D-dimeer waarde in het bloed wordt er een uitspraak gedaan over de kans op het hebben van een trombose. Als de bestaat in de categorie hoge verdenking zit, dan zal er normaal gesproken een twee-punts echo volgen om de diagnose DVT te bevestigen of uit te sluiten. Als er een DVT wordt gezien dan zal de patiënt meestal conservatief behandeld worden met antistolling in de vorm van een laag moleculair gewicht heparine voor 5 dagen en gelijktijdig opstarten van vitamine-Kantagonisten voor een totale duur van 3-6 maanden of levenslang, afhankelijk van de risicofactoren en voorgeschiedenis van trombose. Additioneel wordt er gestart met compressieve zwachtels of een therapeutisch elastische kous voor de totale duur van 2 jaar en de patiënt dient te mobiliseren. Met deze behandeling zal in 20-50\% van de patiënten het post trombotische syndroom ontstaan. PTS is gedefinieerd als een chronische aandoening van veneuze hypertensie na een DVT. Subjectieve symptomen van PTS zijn: jeuk, pijn, kramp, paresthesie of een zwaar gevoel in het been. Objectieve symptomen zijn oedeem, teleangiëctastieën, varices, induratie, hyperpigmentatie, roodheid aan het been of een open wond. Symptomen kunnen variëren van mild, matig tot ernstig. Ernstige PTS is geassocieerd met hoge medische kosten en sociale lasten en heeft een grote impact op de kwaliteit van leven van patiënten. PTS is daarin vergelijkbaar met andere chronische ziekten zoals diabetes mellitus, COPD, en hartfalen. De diagnose PTS wordt gesteld aan de hand van een schaal voor PTS (Villalta schaal) vaak in combinatie met een ziekte specifieke kwaliteit van leven vragenlijst. Een op de vier patiënten kan gecategoriseerd worden als een LET 3 of 4 DVT (iliofemoraal). Een LET 3,4 trombose is geassocieerd met een hoger risico op het ontwikkelen van PTS.

\section{Post trombotisch syndroom}

The exacte etiologie van PTS is niet opgehelderd, maar er is een sterke associatie met obstructie en reflux van het diepe veneuze systeem. Standaard antistolling therapie lost het stolsel niet actief op. Het lichaamseigen lytische systeem is verantwoordelijk voor oplossen van het stolsel en tot het verkrijgen van rekanalisatie in de vene. Als de rekanalisatie van de vene niet toereikend is, zal het resterende deel van het stolsel omgevormd worden tot fibreus weefsel en dit proces resulteert in partiële of volledige obstructie van de vene en zorgt ook voor stijfheid van de vene wand. Gelijktijdige inflammatie kan de kleppen beschadigen en leiden tot veneuze insufficiëntie. Deze mechanismes leiden uiteindelijk veneuze hypertensie en worden gezien als de oorzaak van PTS. PTS is de meest voorkomende complicatie na DVT. Met een gerapporteerde incidentie 
tot $50 \%$ is het belangrijk om patiënten te herkennen die een verhoogd risico hebben om PTS te ontwikkelen en hen alternatieve therapieën aan te bieden die het post trombotisch morbiditeit kunnen verminderen. Preventie van PTS zou bereikt kunnen worden met vroege verwijdering van stolsel door UACDT omdat dit obstructie van de vene minimaliseert en de klepfunctie kan behouden.

\section{Diagnose en behandeling van DVT}

Hoofdstuk twee geeft een overzicht van de beschikbare behandelingen voor patiënten met een iliofemorale trombose en de resultaten met betrekking tot de behandeling. Een korte introductie wordt gegeven omtrent het probleem PTS na DVT, specifiek in LET 3,4 (iliofemorale) DVT patiënten. Als een arts DVT vermoed bij een patiënt dan kan hij de Wells criteria gecombineerd met een ddimeer gebruiken om het risico in te schatten. Dit is algemeen geaccepteerd als standaard zorg om het aantal onnodige echo's te reduceren. The combinatie van en lage Wells score en lage D-dimeer heeft een negatief voorspellende waarde van 97,7\% wat maakt dat het een zeer handige methode is om veilig een DVT uit te sluiten. De diagnose DVT wordt gemaakt met een standaard twee punts-echo. Dit heeft een sensitiviteit van $98,7 \%$ en een specificiteit van 100\% voor DVT boven de knie en $85,2 \%$ sensitiviteit en 98,2\% specificiteit voor onder de knie DVT in vergelijk met de gouden standaard invasieve flebografie. MR-Venografie en CT-Venografie kunnen overwogen worden bij patiënten met een DVT boven de liesplooi. Het risico op PTS in DVT patiënten varieert in de literatuur tussen de $20 \%$ en meer dan $60 \%$ in de LET 3,4 groep. Het risico op het ontwikkelen van een veneus ulcus na DVT varieert tussen de 3$5 \%$. Additionele stolsel verwijderingsmethode laten goede potentie zien wat betreft het verkrijgen van een open vat en potentieel verlagen van PTS. Echter is het bewijs gelimiteerd tot retrospectieve analyses en case reports.

\section{Villalta schaal om PTS te diagnosticeren}

Hoofdstuk drie beschrijft hoe PTS te diagnosticeren en de validatie van de Villalta schaal voor PTS, de opzet en limitaties van de schaal. PTS bestaat uit subjectieve patiënt gerapporteerde klachten en subjectieve zichtbare afwijkingen aan het been. Subjectieve symptomen van PTS zijn: jeuk, pijn, krampen of tintelingen, paresthesie of een zwaar gevoel in het been. De symptomen kunnen continue aanwezig zijn of wisselend van aard. Inspanning zorgt vaak voor verergering van de klachten en rust en hoog leggen zal de klachten reduceren. Objectieve symptomen zijn onder andere teleangiëctastieën, eczeem, varices, oedeem, lipodermatosclerose en een ulcus. Vanwege de verscheidenheid aan presentatie van PTS is het moeilijk gebleken om consensus te bereiken een goede definitie voor PTS te geven. PTS is een syndroom en er bestaat geen gou- 
den standaard om het te diagnosticeren. Een ad hoc bijeenkomst georganiseerd door het european venous forum heeft PTS gedefinieerd als chronische veneuze klachten/afwijkingen secundair na een DVT. In de laatste 20 jaar zijn er een aantal klinisch schalen ontwikkeld om PTS te diagnosticeren. De diagnose PTS kan overwogen worden als de patiënt een DVT heeft gehand en als gevolg hiervan klachten aan het zelfde been ontwikkeld of houdt een aantal maanden na de DVT. Idealiter zou een klinische schaal PTS kunnen vaststellen en daarnaast ook differentiëren tussen milde PTS en ernstige PTS. De schaal moet makkelijk in gebruik zijn en een goede inter en intra-observer betrouwbaarheid hebben, zodat de score reproduceerbaar is en gebruikt kan worden zowel in onderzoek als in de klinische praktijk. De schaal moet breed gedragen en geaccepteerd zijn wereldwijd. Dit moet pooling van resultaten mogelijk maken zodat er sterke bewijskracht beschikbaar wordt. De Brandjes, Ginsberg enVillalta schaal zijn specifieke PTS schalen en hebben zowel klinische tekenen als klachten van de patient in de schaal zitten. Van deze schalen wordt de Villalta schaal gezien als de schaal van keuze voor PTS, gezien het voldoet aan de eerder genoemde eigenschappen en daarnaast heeft het een goede correlatie met de generieke kwaliteit van leven schaal de Short-form 36 en de ziekte specifieke VEINES-qol score. De reproduceerbaarheid en correlatie met de eigen perceptie van de patiënt op de kwaliteit van leven zijn goed. Inter-beoordelaar betrouwbaarheid tussen artsen en verpleegkundigen is zeer goed. De betrouwbaarheid tussen geen PTS en PTS was goed( $\kappa=0.71)$, tussen matige en ernstige PTS zeer goed $(\kappa=1.00)$. beperkingen van de schaal zijn onder andere: gebrek aan intrabeoordelaar betrouwbaarheid, test-hertest eigenschappen en het ontbreken van veneuze claudicatio in de schaal en de beperking voor de mate van veneuze ulceratie.

Selectie van patiënten die geschikt zijn om UACDT te ondergaan

Patiënten selectie is cruciaal om te bepalen wie in aanmerking komt voor UACDT en wie er het meeste baat bij hebben. De typische meest gebruikte parameters zoals stolsel grootte, duur van de klachten en bloedingsrisico worden gebruikt. PTS incidentie na kuit venetrombose is lager dan na een iliofemorale $($ LET3,4) trombose. Hoofdstuk vier evalueert 2 verschillende methodes van gebruik van de LET classificatie en het effect op de mogelijkheid om PTS te voorspellen op de lange termijn.

De let classificatie verdeelt patiënten in 4 verschillende groepen op basis van de stolsel locatie in de acute fase van een trombose. In Hoofdstuk vijf worden de eigenschappen van de LET classificatie besproken met betrekking tot het voorspellen van PTS incidentie en hevigheid en kwaliteit van leven. Er werd gebruik gemaakt van een database van 1338 patiënten van een cohort uit Antwerpen. Overleden patiënten of patiënten die de leeftijd van 80 jaar en ouder 
hadden bereikt werden geexcludeerd van de analyse. In totaal werden 660 patiënten aangeschreven met het verzoek een vragenlijst in te vullen over hun klachten. We gebruikte een aangepast versie van de Villalta schaal. Generiek kwaliteit van leven (SF-36) en ziekte specifieke kwaliteit van leven (VEINESsym/qol) werd bepaald naast de patiënt eigenschappen zoals leeftijd, Body Mass Index, geslacht en ontvangen therapie. Confounders werden uitgevraagd, zoals recidief DVT, compressie therapie, gebruik van antistolling, orthopedische ziekte, perifeer arterieel vaatlijden en follow-up duur. De initiële stolsel locatie uitgedrukt in LET klasse werd gecorreleerd aan de klachten van patiënten, incidentie van PTS en kwaliteit van leven. Van de 660 patiënten hebben 315 patiënten gereageerd en de vragenlijst ingevuld teruggestuurd. Van deze patiënten werden 100 geclassificeerd als LET 1, 108 hadden LET klasse 2 en 107 patiënten LET klasse 3. Ernstige PTS werd gerapporteerd in 18,4\% van patiënten. Univariate analyse op ernstige PTS liet een odds ratio(OR) van 2.0(0.9-4.6) zien tussen LET 1 en LET 2. Tussen LET 1 en LET 3 was er een OR van 3.0(1.4-6.7) op het ontwikkelen van ernstige PTS. In de multivariate regressie analyse liet LET 2 een OR van 1.9(0.7-4.9) zien op het ontwikkelen van ernstige PTS ten opzichte van LET 1 en LET 3 had een OR 3.4(1.3-8.8) op het ontwikkelen van ernstige PTS ten opzichte van LET 1. VEINES-qol score waren significant lager bij hoger wordende LET klasse $(p=0.003)$. De LET classificatie liet een goede correlatie zien wat betreft voorspellen van ernstige PTS en verminderde kwaliteit van leven op basis van de initiële stolsel locatie. De LET classificatie kan goed gebruikt worden om patiënten te selecteren die het meeste kunnen profiteren van additionele behandeling met UACDT.

\section{Contrast versterkte MR-venografie voor acute DVT}

Om succesvol UACDT toe te passen is het zeer belangrijk om patiënten met een acute verse trombose te selecteren. Tot op heden werden hiervoor patiënten symptomen gebruikt. Echter zijn deze vaak misleidend en kan de trombose er al een tijd zitten voordat deze wordt opgemerkt. Dit kan resulteren in falen van UACDT omdat het stolsel al ouder is dan gedacht en het stolsel georganiseerd is. Adequate voorspellen van de stolsel leeftijd zou de potentie hebben om het aantal gefaalde UACDT procedures te verminderen en daarmee ook het risico van de behandeling. Stolsel leeftijd wordt momenteel geschat op basis van de klachten van de patiënt. Andere methodes met duplex onderzoek zijn ook beschreven in de literatuur. MR-venografie is een relatief nieuwe techniek om DVT in beeld te brengen en kan potentieel bruikbaar zijn in het schatten van de stolsel leeftijd. Hoofdstuk zes beschrijft de resultaten van het gebruik van MRvenografie voor acute DVT. In totaal 46 MRV's zijn gemaakt en beoordeeld door 4 beoordelaars. 2 beoordelaars hadden veel ervaring met MRV en veneuze pathologie, the twee overige beoordelaars waren radiologen met veel ervaring 
in beoordelen van MRI, maar niet op veneus gebied. Alle MRV's werden geïnterpreteerd op het niveau van de femoralis communis aan de aangedane zijde. Er werden 5 mogelijke interpretatiemogelijkheden gegeven.: acuut, subacuut, oud, acuut op chronisch of inconclusief. Elke interpretatie was gedefinieerd met een karakteristieke illustratie. Overeenkomst tussen de twee ervaren MRV beoordelaars was excellent met een kappa van $0.969(\mathrm{p}<0.001)$. Overeenstemming tussen de eerste MRV beoordelaar en de twee overige beoordelaars was ook uitstekend met een kappa van $0.824(\mathrm{P}<0.001)$ en $0.821(\mathrm{p}<0.001)$. de interpretatie van acuut, subacuut en oud stolsel was ook gecorreleerd tussen de duur van klachten van de patiënt. Stolsel gedefinieerd als acuut had een gemiddelde klachten duur van 6.5 dagen (range 2-13), subacuut 13 dagen (818 dagen), en oud 22 dagen (15-32 dagen). Deze studie laat zien dat identificatie van MRV specifieke stolsel karakteristieken mogelijk is en dat er een goede inter-observer overeenkomst is. De stolsel karakteristieken op MRV lieten ook een goede correlatie zien met betrekking tot duur van de klachten van de patient. MRV kan hiermee een belangrijk diagnostiek middel worden voor DVT boven de liesplooi.

\section{Uitvoerbaarheid en veiligheid van UACDT}

CDT wordt gebruikt voor de behandeling van iliofemarale DVT en laat goede resultaten zien wat betreft succesvol oplossen van stolsel in de meerderheid van de patiënten. Echter is de behandelingsduur met een mediane duur van 2.4 dagen lang. UACDT is ontworpen om een snellere effectievere oplossing van het stolsel te bereiken in patiënten met een arteriële afsluiting, afsluiting van een bypass, massale longembolie en DVT. In hoofdstuk zeven wordt onze ervaring met het toepassen van UACDT in iliofemorale DVT beschreven. Een retrospectieve analyse is verricht van alle UACDT behandelingen voor iliofemorale DVTs tussen oktober 2008 en januari 2010. In die tijd zijn 12 behandelingen uitgevoerd. Zeven mannelijke en vijf vrouwelijke patiënten werden behandeld. De mediane leeftijd van de groep was 44 jaar (range 5-79 jaar). De diagnose iliofemorale DVT werd gesteld aan de hand van een veneuze duplex in combinatie met ct-venografie of MR-venografie. 7 patiënten hadden een LET 2,3,4 DVT, 3 patiënten een LET 2,3 DVT, een patiënt een LET 2 DVT en een patiënt een LET 4 DVT. UACDT was succesvol in 85\% van de gevallen (11/13), waarvan in 10 gevallen complete lyse ( $>90 \%)$ is bereikt en partiële lyse (50-90\%)in 1 patiënt. Er werden geen longembolieën geobserveerd en 1 bloeding aan de katheter insteekopening. In drie patiënten werd een onderliggende stenose succesvol behandeld met een ballonangioplastiek en stentplaatsing. Vier patiënten hadden een vroege recidief trombose gewijd aan een niet of onvoldoende behandelde onderliggende stenose van het veneuze traject. 
Hoofdstuk acht beschrijft een groter cohort van patiënten die ook behandeld zijn met UACDT voor acute LET3/4 DVT. In deze retrospectieve analyse zitten 37 patiënten met een gemiddelde leeftijd van 42 jaar(range 5-76), die zijn behandeld in Aken of Maastricht. Er waren 20 linkszijdige trombose, 13 rechtszijdige en 4 bilaterale DVT's. De man/vrouw verdeling was 18/19. De meeste patiënten hadden en klachtenduur voor behandeling van tussen de 7 en 13 dagen. De verdeling in LET-klasse was als volgt: een patiënt met LET 2 DVT, 32 patiënten met en LET2,3 DVT en 4 patiënten met een LET 2,3,4 DVT. Volledige stolsel lyse $(>90 \%)$ werd bereikt in $52 \%$ van de patiënten. Partiële lyse (50$90 \%$ ) werd in $43 \%$ bereikt. De overige $5 \%$ liet geen verbetering zien met UACDT en de behandeling werd hierop gestaakt. De gemiddelde behandelingsduur van UACDT was 43 uur tot succesvolle lyse. In $75 \%$ van de linkszijdige DVT's werd het May Thurner syndroom gezien bij beeldvorming. In twee patienten werd een vena cava inferior atresie gezien. In $54 \%$ van de patiënten werd een aanvullende stentplaatsing verricht om onderliggende stenoses te behandelen. Andere additionele interventies die verricht werden zijn het aanleggen van een Arterio Veneuze-fistel en een chirurgische trombectomie. De Kaplan Meyer survival analyse liet een primaire patency zien van $70 \%$ na 1 jaar en een secundaire patency van $87 \%$ na 1 jaar. Complicaties van de behandeling waren in $8 \%$ kleine bloedingen, $3 \%$ grote bloedingen en $5 \%$ andere complicaties, zoals een heparine geinduceerde trombocytopenie. Een klinische longembolie werd opgemerkt. Er werden geen overlijdens gezien. De longembolie trad op twee weken na de UACDT behandeling en wordt niet beschouwd als zijnde gerelateerd aan de behandeling. De duur van de UACDT behandeling is langer dan in de literatuur werd gerapporteerd door Motarjeme en Raabe. Dit is geduid bij een andere selectie methode en logistieke zaken omtrent het plannen van additionele ingrepen en verschil in leeftijd van het stolsel. De tweede belangrijke observatie is het aantal ingrepen nodig voor onderliggende stenoses. In dit cohort in 50\% additioneel behandeld met stents in het veneuze traject. Concluderend vinden wij dat de UACDT behandeling veilig en effectief uitgevoerd kan worden voor patiënten met een iliofemorale DVT. Gerandomiseerde trials zullen deze observatie moeten bevestigen en zullen ook een uitspraak moeten doen over het effect op het voorkomen van PTS na UACDT. De Cavent studie is de eerste gerandomiseerde studie die hiernaar kijkt, maar gebruikt geen UACDT. De behandelingsduur en het bloedingsrisico blifft een continue discussiepunt. De CAVA trial randomiseert patiënten met een iliofemorale DVT tussen conservatieve behandeling en UACDT en kijkt ook naar het klinische uitkomstpunt PTS. De studie loopt momenteel in heel Nederland en includeert patiënten met een LET 3/4 DVT.

In de Verenigde Staten loopt ook een soortgelijk onderzoek. De attract trial vergelijkt verschillend stolsel oplossende technieken versus de conservatieve 
behandeling. Als deze 3 studies verricht zijn zullen de gepoolde resultaten een antwoord moeten geven of additionele stolsel verwijdering zinvol is om PTS te reduceren in patiënten met een iliofemorale DVT.

\section{Stenting en UACDT}

In de vorige twee hoofdstukken wordt gerapporteerd dat $50 \%$ van de patiënten stenting ondergaat van een onderliggende veneuze obstructie. Occlussies van deze stent zijn gezien in een klein percentage van deze patiënten. De meeste patiënten merkten dit door weer een acute zwelling van het been en klachten van een trombose. Met duplex kon de diagnose stentocclusie worden gemaakt. Vanwege de aanwezigheid van vers stolsel is getracht het traject weer open te maken met behulp van UACDT. In de literatuur is weinig te vinden over de aanpak van zulke problemen. In hoofdstuk negen bespreken we onze bevindingen met het herwinnen van patency in de geoccludeerde trajecten. Een retrospectieve analyse van 19 patiënten, die tussen januari 2009 en juli 2014 werden gezien met een stentocclusie werd verricht. Alle patiënten ondergingen UACDT als primaire interventie om het traject te openen. De mediane leeftijd was 43 jaar en $67 \%$ was man. De primaire indicatie voor stentplaatsing was het behandelen van een chronische veneuze obstructie in 12 patiënten en in de andere 6 patiënten het behandelen van een residuale obstructie na UACDT voor acute DVT. In 11 van de 18 patiënten slaagden wij om het traject te openen. Primaire patency was $73 \%$ na 14 maanden follow-up. Na succesvolle UACDT werd er in 7 patiënten overgegaan tot stentplaatsing en in 6 patiënten werd een AV-fistel aangelegd. Mediane UACDT tijd was 19 uur (range 11-101) in de succesvolle behandelingen. Deze behandelingsduur is meer in lijn met de eerdere publicaties over UACDT. In vergelijking met de behandelingsduur in hoofdstuk 8 is de duur meer dan gehalveerd. Dit verschil zit in ervaring en het herkennen van stenoses die therapieresistent zijn. Hierdoor is er eerder gestopt met UACDT en overgegaan tot stentplaatsing of andere additionele behandeling. De patiënt gerapporteerde klachtenduur bleek in dit cohort niet bruikbaar te zijn om succes van de behandeling te voorspellen. De stolsels waren dikwijls ouder dan werd verwacht aan de hand van patiënt gerelateerde klachtenduur. Wat betreft veiligheid van de behandeling. 6 complicaties werden gezien, waarvan 2 kleine bloedingen, 1 klinische relevante bloeding. Een heparine geinduceerde trombocytopenie en 1 allergische reactie werd gezien op jodiumhoudend contrastvloeistof. Er werden geen longembolieën of overlijdens gezien. In onze ogen maakt dit dat de behandeling als veilig kan worden beschouwd. Stent gerelateerde problemen werden herkent als de meest voorkomende oorzaak van stentocclusie. Dit maakt de roep om specifieke veneuze stents luider. Er werd een redelijke primaire patency van $73 \%$ bereikt na succesvolle interventie. Naar aanleiding van deze analyse is een voorstel gedaan 
voor follow-up van patiënten na veneuze stentplaatsing. Het voorgestelde follow-up regime stelt voor de patiënt na 2 en 6 weken, 3 en 6 maanden en daarna jaarlijks terug te laten komen voor controle van het stenttraject middels duplex. Deze intervallen zullen voldoende zijn om de meest voorkomende problemen omtrent stents op tijd te kunnen ondervangen. Prospectieve evaluatie van dit protocol zal zijn effectiviteit moeten bewijzen.

\section{Veiligheid tijdens UACDT}

Om het bloedingsrisico te beperken en daarmee de veiligheid van de patiënt tijdens UACDT te vergroten, wordt er standaard bloedonderzoek gedaan. Fibrinogeen levels zullen niet onder de $1.0 \mathrm{~g} / \mathrm{l}$ moeten vallen, omdat dit de kans op een bloeding ergens in het lichaam laat toenemen. In het geval dat het fibrinogeen onder de $1.0 \mathrm{kwam}$, werd dit gesuppleerd met het geven van fibrinogeen in de vorm van Fresh Frozen Plasma (FFP). In hoofdstuk tien vergelijken we 3 verschillende methodes voor het bepalen van de plasma fibrinogeen hoogte in patiënten die UACDT ondergaan. De standaard methode om dit te bepalen is via de Claus methode. Zowel optische als mechanische methodes zijn beide geaccepteerd en worden gelijkwaardig beschouwd. ROTEM FIBTEM is een alternatieve methode voor fibrinogeen metingen, maar dit is geen standaard methode. We observeerden problemen met fibrinogeen bepalingen in patiënten tijdens UACDT. De optische methode CS2100i en mechanische methode STA-go en functionele analyse middels ROTEM werden met elkaar vergeleken. Van 15 patiënten waren bloedmonsters beschikbaar en analyses op alle 3 de methodes. Zij waren prospectief geïncludeerd in de studie. Een gemiddeld verschil van $0.5 \mathrm{~g} / \mathrm{l}$ (95\% betrouwbaarheids interval $0.05-0.96)$ werd geobserveerd tussen de CS2100i en de STA-go in alle monsters. Naast dit verschil werd er ook opgemerkt dat in $41 \%$ van de monsters het apparaat een sample error aangaf. Dit kwam voornamelijk voor bij de optische meting en nauwelijks bij de mechanische meting. In de ROTEM fibtem analyse was altijd een uitslag beschikbaar. D-dimeer waardes werden vergeleken in de monsters om te bepalen of een hoge d-dimeer waarde een verstoring verzorgde in de optische methode. De monsters met een error hadden een mediane D-dimeer waarde van $54 \mu \mathrm{g} / \mathrm{l}$ (IQR 20.0-91.3) en in de monster zonder error was dit 50 $\mu \mathrm{g} / \mathrm{l}$ (27.5-118.5). Dit liet geen significant verschil zien tussen beide groepen $(\mathrm{p}=0.655)$. in totaal werden in de 15 patiënten 30 FFP's gegeven vanwege lage fibrinogeen waardes. In twee gevallen werd een kleine bloeding geobserveerd, er werden geen grote bloedingen gezien. De studie verheldert echter niet of het verschil in de gevonden methodes een overschatting is van de mechanische methode of een onderschatting van de optische methode. Welke methode de beste is om bloedingsrisico te minimaliseren voor patiënten blijft de vraag. Eerder werd in de 
literatuur aangegeven dat hoge d-dimeer waardes de bepaling zouden verstoren. Dit kunnen wij echter niet terugvinden in onze analyse.

\section{Toekomstig onderzoek}

Hoofdstuk elf geeft een overzicht van de stolseloplossende modaliteiten die momenteel beschikbaar zijn voor de behandeling van iliofemorale DVT. Daarnaast geeft het een overzicht van de vooruitgang die nog geboekt kan worden en de mogelijkheden om de technieken te verfijnen. UACDT is een van die technieken. Er zijn echter al verbeteringen op komst. De stolsel verwijderende technieken moeten de incidentie van PTS zien te verlagen. De techniek moet veilig zijn zodat de patiënt er netto op vooruit gaat. De meeste technieken maken nog gebruikt van trombolytica om het stolsel op te lossen. Echter brengt dit een inherent risico van bloedingen met zich mee, met potentieel verwoestende gevolgen voor de patiënt, zoals een intracraniële bloeding. Puur mechanische trombectomie apparaten moeten zonder het gebruik van trombolytica het stolsel verwijderen en hebben daarmee het risico gepaard gaande met trombolytica gebruik niet. Dit betekent ook dat er meer patiënten potentieel in aanmerking kunnen komen voor deze behandelingsoptie, denk hierbij patiënten met een maligniteit (30\% van alle DVT's). Op dit moment is er echter nog geen puur mechanische techniek beschikbaar die het volledige stolsel kan verwijderen bij een LET3/4 trombose. Vooruitgang in de beeldvorming bij DVT zal het mogelijk maken patiënten beter te selecteren en te voorspellen bij wie trombolyse of trombectomie zinvol is en wat er moet gebeuren aan additionele stentplaatsing bij resterende stenotische afwijkingen. Hiermee zal de effectiviteit toenemen.

De meeste technieken die momenteel gebruikt worden, zijn rechtstreeks geadapteerd vanuit de arteriële behandelingen. Echter zijn de omstandigheden in het veneuze stelsel geheel anders dan de omstandigheden in het arteriële stelsel. Het is dan ook nodig dat er specifiek materiaal beschikbaar moet komen wat inspeelt op de veneuze ziekten. De stents bijvoorbeeld dienen aan een aantal andere voorwaarden te voldoen om goed te functioneren in het veneuze stelsel dan in het arteriële stelsel. Met speciaal ontwikkelde stent hopen we het aantal stent gerelateerde problemen te verminderen en te voorkomen.

De toekomstige trombectomie apparatuur dient te voldoen aan een aantal voorwaarden. Het moet minimaal invasief zijn, het volledige stolsel dient verwijderd te kunnen worden, liefst in 1 sessie, het dient makkelijk in gebruik te zijn en het liefst zo goedkoop mogelijk. De behandeling dient snel te zijn om eventuele stentplaatsing daarna gelijk te faciliteren. Met deze doelen in gedachte hopen we dat er in de toekomst een grotere patiëntengroep behandeld kan worden en dat er meer mensen in aanmerking komen. Momenteel ligt de focus 
bij de LET 3/4 patiënten, maar hier hopen we binnenkort ook de LET 2 patiënten in mee te nemen.

\section{Conclusie en toekomstperspectief}

Samenvattend bespreekt dit proefschrift de minimaal invasieve behandelingsoptie om PTS te voorkomen na iliofemorale DVT (LET3/4). Speciaal LET3/4 omdat dit vaker ernstige PTS veroorzaakt. UACDT in acute DVT kan potentieel PTS verlagen. Dit proefschrift laat zien dat UACDT veilig en uitvoerbaar is in acute LET3/4 trombose en stent trombose. Er is nog ruimte voor verbetering in het voorspellen wie PTS krijgt na DVT. De LET classificatie kan hier een goed instrument voor zijn, wat makkelijk door de radioloog of arts gebruikt kan worden om patiënten te categoriseren. Om succesvolle stolselverwijdering te voorspellen is het belangrijk de leeftijd van het stolsel te bepalen. MRvenografie lijkt hier potentieel een goed diagnosticum voor te zijn. Betere patienten selectie en trombectomie zonder trombolyticum en beter stent materiaal voor veneus moeten ook aan een betere uitkomst bijdragen. De cavent study laat een absolute risico reductie zien in patiënten additioneel behandeld met CDT. Toch zijn er nog steeds zorgen over het bloedingsrisico en het potentiele voordeel voor de patiënt. We hopen dat de gecombineerde resultaten van de drie gerandomiseerde studies een definitief antwoord geven en de zorgen opzij kunnen zetten.

Ook de conservatieve behandeling van DVT is veranderd. Er zijn alternatieve voor de traditionele vitamine-K-antagonisten bijgekomen. Deze alternatieven zoals, dabigatran, rivaroxaban en apixaban worden in de dagelijkse praktijk steeds meer gebruikt. De voordelen in gebruik zijn bekend. Het is echter nog onduidelijk of deze nieuwe middelen iets doen op de incidentie van PTS. Als de middelen langer in gebruik zijn, kan er gekeken worden wat hun invloed op de PTS incidentie is. Het gebruik van een therapeutisch elastische kous na DVT is standaard therapie sinds de jaren ' 90 . In een recente gerandomiseerde studie is echter vergeleken of het dragen van een therapeutisch elastische kous versus een niet therapeutische kous de incidentie van PTS verlaagd na 2 jaar. De studie laat geen verschil zien na 2 jaar. Dit is in tegenspraak met eerder uitgevoerde studies die wel effect van de therapeutisch elastische kous laten zien. In de nabije toekomst zal hier ook aandacht voor moeten zijn om het precieze werkingsmechanisme te achterhalen. Het probleem PTS wordt steeds bekender en men is zich er steeds meer van bewust dat hier iets aan gedaan moeten worden. De roep voor stolsel verwijderende technieken wordt luider. Dit zal zeker gaan leiden tot de ontwikkeling van betere technieken om dit te bewerkstelligen. Wij verwachten dat acute stolselverwijdering binnenkort standaard zorg zal worden. Er zal een grote groep patiënten zijn die in aanmerking zal komen voor 
deze therapieën. Dit zal de fabrikanten ook stimuleren te investeren en te ontwikkelen op dit gebied.

De diagnose en behandeling van DVT is verdeeld onder een aantal specialisten. De internist is de specialist die DVT voornamelijk behandeld. De radioloog speelt een belangrijke rol in de diagnostiek, de dermatoloog kan goed advies geven over de te volgen compressie therapie en in ernstige gevallen kan de chirurg een invasieve behandeling voorstellen vaak in overleg met de radioloog. Met de LET classificatie hopen we ook voor de huisarts een duidelijke stramien uit te zetten zodat zij zouden kunnen bepalen of iemand specialistische hulp nodig heeft. In sommige gevallen kan dan gekozen worden om naar een gespecialiseerd centrum te verwijzen voor veneuze ziektes, waar het hele scala van conservatieve therapie tot stolselverwijdering wordt aangeboden. 


\section{Valorization}




\section{Relevance and economic impact of the scientific results}

This thesis shows there is a large group of patients who experience complaints after standard treatment for DVT. Most physicians are unaware of the longterm complications of DVT, and most physicians are unaware how many patients are affected by PTS. Patients who have PTS are often told, that there are no treatment options, and they should coop with their complaints. This thesis sheds a light on the possibilities of PTS prevention and the magnitude of the problem. Physicians are also unaware of the possibility UACDT for the treatment of DVT. The most heard comment why not to use UACDT is the risk of intracranial hemorrhage. In this thesis we hope to provide more up to date information on the bleeding risk with UACDT in DVT patients. Hopefully this thesis will put bleeding risk in a new perspective so physicians will more likely consider UACDT.

As mentioned earlier in this thesis, PTS is associated with high healthcare costs. Especially severe PTS causes the highest medical costs. If PTS can successfully be prevented by UACDT it will eventually reduce costs for society, as severe PTS is also associated with many lost workdays.

\section{Groups of interest outside the medical field}

Internists, radiologist, vascular surgeons, general practitioners and dermatologists are targeted in this thesis. Pharmaceutical companies can find new information on how to target their therapies. There is a great potential in optimizing treatment for DVT patients. Current devices are directly adapted from the treatment of arterial diseases. This thesis shows a potential area for future developments and also shows how many patients could be eligible for additional treatments. This big potential could awaken the commercial industry to dedicate research funds towards venous diseases and develop dedicated venous devices as is also discussed in chapter eleven.

\section{Activities and implementation as result of this thesis}

The LET classification is a novel tool, which can be used to identify patients with a high risk for developing severe PTS. The classification has to be evaluated prospectively in other to become useful in daily clinical practice. When using the LET-classification, the physician will have to be aware of the exact location of the thrombus. Duplex ultrasound and/or MR-venography can provide the clinician with this information. MR-venography is used in all DVT patients, and we believe it should be considered in all DVT patients with an iliofemoral (LET3/4) DVT. To show the possible advantages of MR-venography compared 
to duplex ultrasound, there is currently a study comparing those two modalities with the golden standard invasive venography. The results are currently analyzed and hopefully published in the near future.

Another novelty was used in the analysis of the LET classification. The modified version of the villalta scale made it possible for the patients to fill out the score on their own. Previously scored villalta scores depended on the physician to provide information on the clinical signs on the leg of the patient. With the addition of a visual guide, the patient was able to score the clinical signs themselves. This will simplify future evaluations of PTS in large group of patients, and does not necessitate a visit to the hospital.

\section{Innovative ideas and activities as result of this thesis}

The chapters on UACDT add to the already existing knowledge, that UACDT is a technique for thrombus removal in the acute phase of DVT. In the Netherlands there is still great reluctance to treat patients with UACDT for DVT. This resulted in low number of patients being referred for the randomized controlled trial currently conducted in the Netherlands.

The modified version of the Villalta scale used in chapter four is new and could potentially make follow-up of large patient groups easier. The modified version had been used previously by Wik et al. and we further adapted the score to be more reliable. The original score necessitates that the physician examines the leg of the patient in order to come to a score. With the modified version the leg examination is done by the patients or his partner. This reduces the need for a hospital visit, reduces traveling costs and saves time of the physician, while retaining reliable results.

Treatment of stent problems have not been described in literature before, this is the first report ever on the topic. Previous reports by Raju, Neglen, Titus and de Wolf, describe the use of stents in the venous system. Beakgaard, Enden and our own experience show stent placement is sometimes necessary after DVT. Stent problems are reported, however a treatment option for these complications is never reported. In this thesis there is a first dedicated paper on these problems and we encourage other centers to also share their experience with the world.

\section{Planning and realization of future activities as result of this thesis}

Results of randomized controlled trials are still needed to provide us with conclusive answer in the subject of PTS prevention with UACDT. The CAVA-trial already started in 2010 and is still enrolling patients at the moment of writing. 
Results of the trial are expected in 2017/2018. Depending on the outcome of this trial in combination with the Attract-trial and CaVenT-study, the trial will probably be succeeded by another randomized trial comparing different thrombus removal techniques. These trials will likely start in 2017 or 2018.

During the UACDT treatment, we encountered many stenotic leasions in the venous tract, and especially in the common iliac vein. Research on the best treatment options for these kinds of lesions will be necessary to ensure optimal patient care. Different stents and approaches should be compared to see which is the best. 


\section{Dankwoord}


Een proefschrift komt niet uit zichzelf tot stand. In de loop van een proefschrift raken er een heel aantal mensen betrokken. Ik wil de gelegenheid aangrijpen om een aantal hiervan persoonlijk te bedanken.

Prof. dr. Wittens, beste Cees, jij was net een jaar in Maastricht toen ik me bij het team mocht voegen. Toen bestond het team nog uit 5 personen. In de loop van de tijd is het team ongelofelijk uitgebreid. Wat ik het meeste waardeer is dat je ons (promovendi) heel veel kansen hebt gegeven om artikelen te schrijven, congressen te bezoeken en presentaties te geven. Het enige wat wij hoefden te doen was de kansen aan te grijpen. Naast het promoveren vind je het ook heel belangrijk dat iedereen daarna op de juiste plek terecht komt en daar help je dan ook graag in mee. Dit waardeer ik ontzettend. Ik heb veel geleerd in deze periode en daar ben ik jou veel dank voor verschuldigd.

Prof. dr. ten Cate, beste Hugo, jij stond tijdens mijn promotie wat meer op de achtergrond. Tijdens overleg met het promotieteam ging het er soms verhit aan toe, maar jij was dan de rust zelve en zorgde ervoor dat we met een werkbaar voorstel de kamer verlieten. Bij moeilijke vraagstukken over patiënten met stollingsproblemen kon ik altijd bij je terecht.

Dr. ten Cate-Hoek, beste Arina, toen ik de cava-studie overnam van Stephanie kwam ik bij jou terecht. Bedankt voor je inzet voor mijn proefschrift en de cavastudie. Dank ook voor je hulp bij statistiekvraagstukken en praktische adviezen omtrent het onderzoek. Je kon Cees soms het broodnodige tegengas geven.

Irma en Margriet, in het begin moest ik de studie alleen gaande houden. Gelukkig kreeg ik naar verloop van tijd hulp van jullie. Margriet, jouw ervaring met trials en monitoring heeft ontzettend veel bijgedragen aan de cava-studie. Samen met Irma vorm je een goed team om patiënten goed voor te lichten over de cava-studie en om de deelnemende centra op de hoogte te houden en te zorgen dat ook daar alles in orde is. Met jullie erbij werd het niet alleen beter, maar ook een stuk gezelliger.

Irwin, jou wil ik graag bedanken voor alle echo's die je hebt gemaakt en nog steeds maakt voor alle patiënten. Als er weer eens een cava patiënt tussendoor kwam, dan kreeg ik alsnog de echo die ik nodig had. Daarnaast heb je mij veel geleerd over echo's en hebben we veel gepraat over artikelen en mogelijkheden voor nieuw onderzoek. 
Carina, jij zit al vanaf het eerste uur bij het cava-team. In het begin heb je me vaak geholpen bij het prikken van bloed bij patiënten. Daarnaast heb je ook goed geholpen bij het inplannen van studiepatiënten.

De leescommissie bestaande uit Prof. dr. Steylen, prof. dr. De Haan, prof. dr. M. Huisman, dr. R. Rennenberg, en dr. E. Serné wil ik graag hartelijk bedanken voor hun tijd om mijn proefschrift te beoordelen en bij de verdediging hiervan aanwezig te zijn.

Tijdens mijn promotie onderzoek was Yee Lai een van mijn kamergenoten. We hebben vaak gelachen tijdens de koffie en lunchpauzes en hebben ook veel meegemaakt op congresbezoek. Deze belevenissen zullen mij nog lang bijblijven. Veel succes met je opleiding tot dermatoloog. Ik kijk ook uit naar jouw proefschrift.

Tijdens het eerste jaar van mijn promotie zat ik nog op een studentenkamer. Daar kwam verandering in toen Fabio zich bij het veneuze team voegde. Fabio had een groot appartement in Ceramique uitgezocht, maar zocht nog huisgenoot om de maandelijkse kosten draagbaar te maken. Ik heb me toen geen moment bedacht en ben in het appartement ingetrokken. Ik heb daar tot mijn verhuizing naar Rotterdam gewoond en erg veel plezier van gehad. Ik dank Fabio ook voor zijn unieke aanwezigheid en gezelligheid bij de borrels en etentjes bij cafe Sjiek en op congres.

Rick, jou wil ik bedanken voor je expertise en je toewijding ten aanzien van de veneuze patiënt. Het meedenken en kritisch reviseren van artikelen en je hulp om het mogelijk te maken om vandaag toch nog even die patiënt te helpen in plaats van morgen.

Carsten, ik heb meegemaakt hoe je van assistent naar radioloog bent gegaan en uiteindelijk als interventie-radioloog in Venlo aan de slag bent gegaan. Voor de cava-studie en voor mij heb je veel betekend wat betreft het beoordelen van MRV's. Daarnaast gaf je mij ook goed advies omtrent het promoveren en levenszaken. Een avond die me nog goed bijstaat was de vrijdag na hemelvaart waarop we overdag nog gewerkt hebben aan onderzoek om daarna culinair te genieten bij manjefiek samen met Mark. Veel succes in Venlo en veel geluk met Jette en jullie twee kinderen.

Jorinde, Jolanda, Cora en Wijnand, jullie ook bedankt voor al jullie inzet omtrent mijn proefschrift en de leuke tijd die ik in Maasticht heb gehad. 
Om alle data te kunnen verzamelen en alles omtrent de patiënten te regelen heb ik ook hulp gehad van studenten. Milea en Claudia, dank voor jullie inzet. Jullie hebben kunnen zien wat onderzoek doen inhoudt en wellicht heeft het jullie gestimuleerd om ook een keer onderzoek te gaan doen.

Daarnaast wil ik de verpleging van D4 bedanken voor het waken over de patienten die aan de trombolysekatheter liggen. Ik wil in het bijzonder André, Ria en Yuri bedanken. Dank voor het steeds op korte termijn ruimte maken voor patiënten van de cava studie.

Ralph, ik was zeer verheugd toen ik hoorde dat jij naast een goede vriend ook een collega van mij werd. We hebben mooie tripjes gemaakt, waarvan New Orleans wel de meest memorabele is. Daarnaast heb je een mooi onderzoek uitgevoerd en ik hoop dat je hier snel op kunt promoveren. Veel succes in jouw toekomstige carriere in de gynaecologie. Ik ben benieuwd waar dit avontuur je gaat brengen.

Naast alle collega's en vrienden in de medische wereld zijn er ook vrienden die helemaal niks met geneeskunde te maken hebben en al voor de studie aanwezig waren. Ron, Bart, Rob A., Joost, Benno en Maarten, dank voor jullie steun tijdens het promoveren, de verjaardagen, bruiloften, uitjes en 'sjaele zeiver'.

De oud-Reflex groep bestaande uit Irene en Sander, Tineke en Lars, Hanneke en Marijn, Lonneke en Wenzel, Frans en Miranda, Koen en Marloes, David en Linda, Yvo en Kevin wil ik ook graag danken voor de continue steun, interesse in mijn bezigheden en fijne weekendjes weg. Ik hoop dat er nog velen zullen volgen.

Daarnaast wil ik heel het dispuut e Causa Ignota bedanken en in het speciaal de volgende personen: Rik, Marco, Etienne, Jeroen, Yves, Marc Jenniskens, Marc Thijssen, Nick, Stijn. Daarnaast ook de huidige generatie en de generaties die nog gaan komen. Ik vond het heel speciaal om tijdens de promotieperiode het dispuut nog van dichtbij te kunnen volgen en te zien hoe het van een groepje oprichters is uitgegroeid naar de huidige groep enthousiastelingen.

Ad en Anke den Boer wil ik graag bedanken. Jullie hebben mij met open armen ontvangen en ik voel de warmte binnen jullie familie heel erg goed. Anke, heel erg bedankt voor het ontwerpen van de mooie, creatieve kaft en uitnodigingen. Ook Jetske en Jors wil ik graag bedanken voor alle gezelligheid en warmte en veel geluk wensen samen met Timo. 
Dan ben ik aangekomen bij het gedeelte waar ik mijn paranimfen mag bedanken.

Suzanne, mijn kleine zusje. Je weet het wellicht niet, maar recent heb jij mij geinspireerd om een moeilijke keuze te maken. Ik heb gezien hoe jij nu je plek hebt gevonden op je werk en hoe dit niet altijd even makkelijk ging. Ik vind het ontzettend leuk dat jij paranimf wilt zijn en naast mij bij de verdediging staat. Ik wens je heel veel geluk toe in de toekomst.

Mijn tweede paranimf is Mark de Wolf, studievriend vanaf de eerste dag van de opleiding geneeskunde in Maastricht. Ik weet nog goed hoe we na het allereerste feest achterop de fiets zaten bij Joris en Fabian en dat we blij waren dat we de eerste avond ongeschonden aan kwamen bij ons slaapadres. Ik zou een compleet hoofdstuk kunnen schrijven over alle avonturen die hierop volgde, maar ik hou het beperkt. Bestuur van MFV Relfex en mede-oprichters van e Causa Ignota zijn twee dingen die we beiden hebben gedaan. Daarnaast hebben we samen verschillende festivals bezocht. Tijdens de coschappen zaten we in verschillende ziekenhuizen waardoor het contact wat minder frequent werd dan voorheen. Dit veranderde echter drastisch toen we in ons laatste jaar beiden bij Nicole Bouvy op de chirurgie belandden. Daarna ben ik als eerste bij Cees beland en jij volgde al snel. Als collega's hebben we ook veel samen meegemaakt, zoals congressen bezoeken, workshops organiseren, poli draaien, APG's maken en nog veel meer. Het feit dat jij ook bij Cees werkte, heeft ervoor gezorgd dat ik bijna altijd een sparringpartner beschikbaar had. Ik wil je graag bedanken voor je vriendschap, je kritische opmerkingen en soms andere manier van denken. Ik wens je veel succes met jouw vervolg carrière en ik hoop snel bij jouw promotie aanwezig te mogen zijn.

Mijn beide ouders wil ik uiteraard ook graag bedanken. Promoveren is iets aparts en soms moeilijk te begrijpen of uit te leggen. Ik ben niet iemand die makkelijk vertelt wat me bezig houdt en zeker niet over zaken waar ik het moeilijk mee heb. Dit zal niet altijd even gemakkelijk geweest zijn, aangezien ouders hun kinderen graag willen helpen. Ik heb nooit de behoefte gevoeld om hulp van jullie te krijgen. Ik ben al tevreden puur met het feit dat jullie er beiden zijn en dat ik weet dat ik kan terugvallen op jullie ook al hoop ik dat nooit te hoeven doen. Weet dat jullie heel veel voor mij betekenen.

Femke, het laatste woord richt ik aan jou. Jij ben in het laatste jaar van mijn promotie volledig in mijn leven gekomen. Ik kende je weliswaar al langer, maar vanaf de jaarwisseling 2013-2014 is dit heel anders geworden. Jij hebt vooral mijn laatste loodjes van het proefschrift meegemaakt. Dit heeft langer geduurd dan van te voren gehoopt. Jij bent een onvoorwaardelijke steun voor mij. Ik 
merk dat we in een aantal opzichten op elkaar lijken en dat we elkaar op een aantal punten goed weten aan te vullen. Het samenwonen in Rotterdam bevalt me uitstekend en dat komt door jou. Je hebt me ook gezien in de fase na het proefschrift. Je hebt gezien dat ik op de interne niet op mijn plek zat en je hebt me onvoorwaardelijk gesteund in het zoeken van een nieuwe richting waarin ik gelukkig wordt en daarmee jij ook. Jij bent een goede steun voor mij geweest en ik wil dit ook voor jou zijn. Ik wil een mooie toekomst opbouwen en gelukkig worden met jou. Ik houd van jou leive sjat! 


\section{List of publications}

Strijkers RHW, de Wolf MAF, Arnoldussen CWKP, Timbergen MJM, de Graaf R, Cate-Hoek ten AJ, et al. Venous in-stent thrombosis treated by ultrasound accelerated catheter directed thrombolysis. European Journal of Vascular \& Endovascular Surgery. 2015 Apr;49(4):440-7.

Strijkers RHW, Arnoldussen CWKP, Wittens CHA. Validation of the LET classification. Phlebology. 2015 Mar;30(1 Suppl):14-9.

Arnoldussen C, Strijkers RHW, Lambregts D, Lahaye M, de Graaf R, Wittens C. Feasibility of identifying deep vein thrombosis characteristics with contrast enhanced MR-Venography. Phlebology. 2014 May 19;29(1 suppl):119-24.

Strijkers RHW, Arnoldussen CW, Wittens CH. Thrombectomy without lysis: the future? Phlebology. 2014 May 19;29(1 suppl):125-34.

Strijkers RHW, Wittens CH. De invasieve behandeling van diepveneuze trombose ter voorkoming van post trombotisch syndroom. Nederlands Tijdschrift Voor Dermatologie En Venereologie. 2014 May 6;24(3):159-63.

Strijkers RHW, Grommes J, Arnoldussen CW, de Graaf R, Cate ten AJ, Wittens $\mathrm{CH}$. Ultrasound-accelerated catheter-directed thrombolysis in acute iliofemoral deep venous thrombosis. Journal of Vascular Surgery: Venous and Lymphatic Disorders. Elsevier; 2013.

Witteman BPL, Strijkers RHW, de Vries E, Toemen L, Conchillo JM, Hameeteman W, et al. Transoral incisionless fundoplication for treatment of gastroesophageal reflux disease in clinical practice. Surg Endosc. 2012 May 31.

Strijkers RHW, Cate-Hoek ten AJ, Grommes J, Arnoldussen CWKP, Wittens CHA. [Catheter-directed thrombolysis in iliofemoral deep-vein thrombosis]. Ned Tijdschr Geneeskd. 2012;156(36):A4759.

Strijkers RHW, Wittens CHA, Kahn SR. Villalta scale: goals and limitations. Phlebology. 2012;27 Suppl 1:130-5.

Grommes J, Strijkers RHW, Greiner A, Mahnken AH, Wittens CHA. Safety and feasibility of ultrasound-accelerated catheter-directed thrombolysis in deep 
vein thrombosis. European Journal of Vascular \& Endovascular Surgery. 2011 Apr;41(4):526-32.

Botden S, Srijkers RHW, Fransen S, Stassen L, Bouvy N. The use of curved vs. straight instruments in single port access surgery, on standardized box trainer tasks. Surg Endosc. 2011 Mar 25.

Strijkers RHW, ten Cate-Hoek AJ, Bukkems SFFW, Wittens CHA. Management of deep vein thrombosis and prevention of post-thrombotic syndrome. BMJ. 2011;343:d5916.

Strijkers, RHW, de Wolf, M., Mahnken, A. H., Wittens, C.; Ultraschallunterstützte Thrombolyse mit dem EKOS-System zur Behandlung der tiefen Venenthrombose Grommes, J. Vasomed, 2011;23(Kongressausg.):16; 0942-1181

RHW Strijkers, J. Grommes, A.J. ten Cate-Hoek, H. Ten Cate, C.H.A. Wittens; Kathetergesteuerte thrombolyse bei patienten mit iliofemoraler tiefer venenthrombose und in-stent-thrombose- klinische erfahrung; Vasomed 2011 ausgabe 5 pagina 236

Mark A.F. de Wolf, RHW Strijkers, Rick de Graaf, Cees H.A. Wittens; Recent developments in the management of the post-thrombotic leg and venous compression syndromes; Turkish Journal of Vascular Surgery 2013;22(2):155-60

RHW Strijkers, Gerhardus J. A. J. M. Kuiper, Rene van Oerle, Rick Wetzels, Arina J. ten Cate, Hugo ten Cate, Cees H.A. Wittens, Yvonne Henskens. Fibrinogen levels in patients under-going thrombolytic therapy for acute iliofemoral deep venous thrombosis are extremely method dependent. Under revision

RHW Strijkers and CHA Wittens; Bookchapter; Thrombolytic management of DVT. Highlights of vascular surgery 2010-2011; editor Alun Davies

RHW Strijkers and CHA Wittens; Bookchapter; Management of iliofemoral DVT; Highlights of vascular surgery 2011-2012; editor Alun Davies

CHA Wittens and RHW Strijkers; chapter19; The acutely swollen leg; A Companion to Specialist Surgical Practice Vascular and Endovascular Surgery; Saunders Elsevier 2014, ISBN 978070204958 3: (pages 334-345) 


\section{Curriculum vitae}

Rob Strijkers is op 1 oktober 1986 geboren te Sittard. In 2004 heeft hij zijn diploma gehaald aan het Gymnasium van de Trevianum scholengroep te Sittard. Aansluitend is hij begonnen met de opleiding geneeskunde aan de Universiteit van Maastricht. In augustus 2010 studeerde hij af en aansluitend is hij begonnen met zijn promotie onderzoek aan dezelfde universiteit onder de begeleiding van Professor dr. C.H.A. Wittens, Professor dr. H. Ten Cate en dr. Arina ten Cate-Hoek op het gebied van de veneuze chirurgie, specifiek de invasieve behandeling van trombosebenen en het postthrombotisch syndroom. Zijn proefschrift is het resultaat van het werk verricht op deze afdeling. Vanaf januari 2015 is hij gestart met de opleiding interne geneeskunde in het HAGA ziekenhuis te den Haag (opleider dr. M. van Buren). De interne geneeskunde bleek echter niet het vak te zijn waarin het gelukkig zou worden. Vanaf 01-01-2016 start hij met een nieuw avontuur, waarin is nog de vraag. 